In presenting the dissertation as a partial fulfillment of the requirements for an advanced degree from the Georgia Institute of Technology, I agree that the Library of the Institute shall make it available for inspection and circulation in accordance with its regulations governing materials of this type. I agree that permission to copy from, or to publish from, this dissertation may be granted by the professor under whose direction it was written, or, in his absence, by the Dean of the Graduate Division when such copying or publication is solely for scholarly purposes and does not involve potential financial gain. It is understood that any copying from, or publiçation of, this dissertation which involves potential financial gain will not be allowed without written permission.

$7 / 25 / 68$ 


\title{
AN EXPERIMENTAL INVESTIGATION OF A JET ISSUING FROM A WING IN CROSSFLOW
}

\section{A THESIS}

Presented to

The Faculty of the Division of Graduate

Studies and Research

by

William Theodore Mikolowsky

\author{
In Partial Fulfillment \\ of the Requirements for the Degree \\ Doctor of Philosophy \\ in the School of Aerospace Engineering
}

Georgia Institute of Technology

May, 1972 


\section{AN EXPERIMENTAL INVESTIGATION OF A JET ISSUING FROM A WING IN CROSSFLOW}

\section{Approved:}

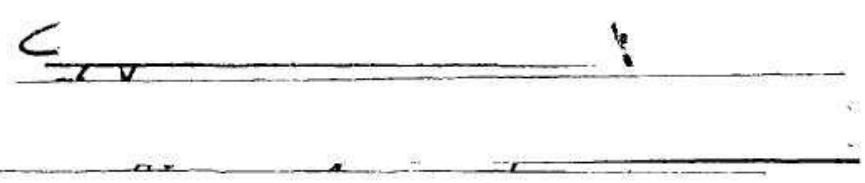

Date approved by Chairman: May 18, 1972 


\section{ACKNOWLEDGEMENTS}

I would like to sincerely thank Dr. Howard M. McMahon for his suggestion of the thesis topic and for his guidance and encouragement throughout the course of this investigation. I am certain that the privilege of working with Dr. McMahon will be a valuable asset in my professional career.

I am indebted to Professor John J. Harper, Professor James E. Hubbart, and $\mathrm{Dr}$. James $\mathrm{C}$. Wu for their critique of the original manuscript and their helpful suggestions. The efforts of Dr. G. T. Colwell and Dr. P. V. Desai in their reading of the original draft are also appreciated.

Mr. John G. Palfery provided a wealth of technical expertise which aided all phases of the experiment and his help is gratefully acknowledged.

The model fabrication and instrumentation would not have been possible without the help of many people. I am particularly indebted to $\mathrm{Mr}$. Calvin $\mathrm{H}$. Wiser for his skillful machining of many of the model components and his assistance in assembling the model, and to Mr. Hendrick R. Hudson for his aide in the installation of the model in the wind tunnel and for his operating the tunnel throughout the test program. I also express my thanks to Mr. Dewey Ransom, Mr. Harold Myers, Mr. John Caudell, Mr. John C. Handley, Mr. Robert A. Cassanova, Dr. James I. Craig, and Mr. Thomas Christian for their help in various phases of the research.

The assistance of Mr. Wayne Novak of the Engineering Experiment Station 
in providing access to the numerically controlled milling machine is appreciated. Also, the efforts of the staff of the Rich Electronic Computer Center should not go unmentioned.

To my mother, Mrs. Emma Mikolowsky, who has provided encouragement throughout my education goes my deepest gratitude. Finally, I thank my wife, Barbara, and my daughter, Laura, for their love, patience, and understanding during the many years we have been in school. 
TABLE OF CONTENTS

\begin{abstract}
Page
ACKNOWLEDGEMENTS . . . . . . . . . . . . . . . ii

LIST OF TABLES. . . . . . . . . . . . . . . . . . vi

LIST OF ILLUSTRATIONS . . . . . . . . . . . . . . . . . . . . . . vii

NOMENCLATURE. . . . . . . . . . . . . . . . . . . . . . . . xvii

SUMMARY .....................

CHAPTER

I. INTRODUCTION . . . . . . . . . . . . . . . 1

Literature Review

Research Objective and Scope

II. EQUIPMENT AND INSTRUMENTATION . . . . . . . . . . . . 8

Wind Tunnel

Air Supply

General Test Arrangement

Two-Dimensional Model

Plenum Chamber and Jet Nozzles

Pressure Measurement Instrumentation

Force Measurement System

Flow Visualization

III. TESTING PROCEDURE AND ACCURACY . . . . . . . . . . 22

Setting of 'Test Conditions

Surface Pressures

Force Measurements
\end{abstract}

IV. DISCUSSION OF THE RESULTS ............

Jet-Off Aerodynamic Characteristics

Jet-On Aerodynamic Characteristics

Comparison with Flat Plate Results 
TABLE OF CONTENTS (CONTINUED)

CHAPTER

Page

Parametric Comparison of the Aerodynamic

Interference Characteristics

Physical Interpretation

Comparison with Theoretical Results

V. CONCLUSIONS . . . . . . . . . . . . . . . . 54

VI. RECOMMENDATIONS . . . . . . . . . . . . . . . . 57

Present Configuration

Modifications for Additional Configurations

APPENDIX

A. CHARACTER OF NOZZLE FLOWS . . . . . . . . . . . . 105

Procedure

Jet Exit Velocity Profiles

Quality of the Free Jet

B. CALIBRATION OF TEST INSTRUMENTATION

AND TEST FACILITY . . . . . . . . . . . . . . . . . . 118

Wind Tunnel Balance System

Internal Balance

Pressure Measurement System

Wind Tunnel Freestream

C. INTERFERENCE SURFACE PRESSURE

DISTRIBUTIONS.

D. INTERFERENCE LIFT, DRAG, AND PITCHING MOMENT COEFFICIENTS . . . . . . . . . . . . . 207

LITERATURE CITED . . . . . . . . . . . . . . . . . . . . 222

OTHER REFERENCES. . . . . . . . . . . . . . . . . . . . . . . 225

VITA. . . . . . . . . . . . . . . . . . . . 226 


\section{LIST OF TABLES}

Table

Page

1. Coordinates of the Modified NACA 0021

Profile . . . . . . . . . . . . . . . . . .

2. Standard Freestream Indicated Velocities

Used for Each $\lambda$. . . . . . . . . . . . . . . . . . .

C-1a. Sample Computer Printout of Surface

Pressure Coefficients . . . . . . . . . . . . . 145

C-1b. Sample Computer Printout of Surface

Pressure Coefficients . . . . . . . . . . . . . 146

C-1c. Sample Computer Printout of Surface

Pressure Coefficients . . . . . . . . . . . . . . . 147

C-1d. Sample Computer Printout of Surface

Pressure Coefficients . . . . . . . . . . . . . . 148

C-2. Summary of Interference Surface Pressure

Distributions . . . . . . . . . . . . . . . . .

D-1. Sample Computer Printout of Interference

Force and Moment Data . . . . . . . . . . . . . 209 


\section{LIST OF ILLUSTRATIONS}

Figure

Page

1. General Test Arrangement . . . . . . . . . . . . . . 60

2. Model as Installed in Tunnel

(View Looking Upstream) . . . . . . . . . . . . . . . . . . . 61

3. Wing Endplate .................... . 61

4. Wing Assembly . . . . . . . . . . . . . . . . 62

5. Wing with Skin Panels Removed . . . . . . . . . . . . . 63

6. Typical Center Wing Skin Panel . . . . . . . . . . . . 63

7. Plenum Chamber Assembly . . . . . . . . . . . . . . . . . 64

8. Plenum Chamber with Upper Surface

Removed . . . . . . . . . . . . . . . . . 65

9. Typical Jet Nozzles . . . . . . . . . . . . . . . . 65

10. Installation of the Plenum Chamber in Wing . . . . . . . . . . . . . . . . . . . . . . . . 66

11. The Installed Plenum Chamber (Wing Panel Removed, View Looking Downstream) . . . . . . . . . . 67

12. Wing Lower Surface with Three Inch Diameter Jet Installed (View Looking Downstrearn) . . . . . . . . . . . 67

13. Location of Surface Pressure Taps . . . . . . . . . . . . . 68

14. Pressure Lines and Scanivalve Mounted on External Plenum Chamber . . . . . . . . . . . . . . . . .

15. Operator's Area and Pressure Measurement Equipment 
LIST OF ILLUSTRATIONS (CONTINUED)

Figure

Page

16. Plenum Chamber Attachment to Floating

Rib on Flexures. . . . . . . . . . . . . . . . . . 70

17. Strain Gages Mounted on Flexures . . . . . . . . . . . . . . 70

18. Automated Procedure for Acquiring

Surface Pressures . . . . . . . . . . . . . . . . . . 71

19. Repeatability of Interference Surface

Pressure Contours . . . . . . . . . . . . . . . . 72

20. Interference Surface Pressure Contours

for Different Freestream Velocities. . . . . . . . . . . . 73

21. Automated Procedure for Acquiring

Force Data . . . . . . . . . . . . . . . . . . . . . . . . . 74

22. Repeatability of the Interference Lift

Coefficient Data . . . . . . . . . . . . . . . . . . 75

23. Lower Surface Spanwise Pressure

Distributions for $\alpha=0$ at Chord Station

6.75 Inches $(x / c=.45)$ Showing Effect of

Transition Strip. . . . . . . . . . . . . . . . .

24. Lower Surface Chordwise Pressure Distri-

bution for $\alpha=0^{\circ}$ at Wing Centerline for

Different Tunnel Speeds . . . . . . . . . . . . . . . .

25. Lower Surface Spanwise Pressure Distributions at Chord Station 6.75 Inches $(\mathrm{x} / \mathrm{c}=.45)$ for $\mathrm{V}_{\mathrm{i}}=100 \mathrm{ft} / \mathrm{sec}$

26. Jet-off Chordwise Pressure Distributions at

Wing Centerline for Different Angles of Attack . . . . . . . .

27. Basic Wing Aerodynamic Coefficients for the Model.

Wing Lift Coefficients for Various Tunnel Speeds. . . . . . . . . . . . . . . . . . . . . . . . . 


\section{LIST OF ILLUSTRATIONS (CONTINUED)}

Figure

Page

29. Wing Drag Coefficient for Various

Tunnel Speeds . . . . . . . . . . . . . . . . . . 82

30. Oil Flow on Upper Surface at $\alpha=9^{\circ}$

and $\lambda=0 .$. . . . . . . . . . . . . . . 83

31. Oil Flow Exhibiting Laminar Separation

Bubble on the Upper Surface at $\alpha=9^{0}$. . . . . . . . . . 84

32. Lower Surface Chordwise Pressure Distri-

butions at Span Station 5.50 Inches for $\alpha=0^{0}$

with and without Jet Operation . . . . . . . . . . . . . . 85

33. Lower Surface Chordwise Pressure Distri-

butions for $\alpha=0^{0}$ and $\lambda=8$. . . . . . . . . . . 86

34. Lower Surface Spanwise Pressure Distri-

butions at Chord Station 6.75 Inches

(x/c $=0.45)$ for $\alpha=0^{\circ}$ and $\lambda=8$. . . . . . . . . . 87

35a. Interference Surface Pressure Contour's for

the Wing and for a Flat Plate . . . . . . . . . . . . 88

35b. Interference Surface Pressure Contours for

the Wing and for a Flat Plate . . . . . . . . . . . . . . . . 89

35c. Interference Surface Pressure Contours for

the Wing and for a Flat Plate . . . . . . . . . . . . . 90

36. Oil Flow for the 3,0 Inch Diameter Jet at

$\lambda=4$ and $\alpha=0$.................... 91

37. Oil Flow on a Flat Plate with a 2.0 Inch

Diameter Jet at $\lambda=4$ (From Reference 15) . . . . . . . . . . . 92

38. Typical Variation of Interference Lift

Coefficient with Angle of Attack . . . . . . . . . . . . . . . . 93

39. Typical Behavior of the Interference Drag

Coefficient and the Interference Pitching

Moment Coefficient. . . . . . . . . . . . . . . . . . . . . 


\section{LIST OF ILLUSTRATIONS (CONTINUED)}

Figure

Page

40. Typical Variation of Interference Lift Coefficient with Jet Exit Location . . . . . . . . . . . . . . . 95

41. Typical Variation of Interference Lift Coefficient with Jet Exit Size . . . . . . . . . . . . . . . 96

42. Oil Flow for the 3.0 Inch Diameter Jet at $\lambda=2$ and $\alpha=0^{\circ}$. . . . . . . . . . . . . . 97

43. Oil Flow for the 3.0 Inch Diameter Jet at $\lambda=2$ and $\alpha=9^{\circ}$............... . 98

44. Chordwise Pressure Distributions for $x_{j} / c=0.65, d_{j} / c=0.10$, and $\lambda=2$ at $\alpha=6^{\circ} . . . . . .$. .

45. Comparison of the Chordwise Interference Pressure Distribution of the Present Model at the Wing Centerline with that of a TwoDimensional Symmetric Elliptical Airfoil Equipped with a Pure Jet-Flap (Reference 34) . . . . . . . . . . 100

46a. Comparison of the Wing Interference Surface Pressure Contours with the Theoretical Calculations of Wooler . . . . . . . . . . . . . . 101

46b. Comparison of the Wing Interference Surface

Pressure Contours with the Theoretical Calculations of Wooler . . . . . . . . . . . . . . 102

46c. Comparison of the Wing Interference Surface Pressure Contours with the Theoretical Calculations of Wooler . . . . . . . . . . . . . 103

47. Comparison of the Wing Interference Surface Pressure Contours with the Theoretical Calculations of Wasson and Kao . . . . . . . . . . . . . 104

A-1. Free Jet Exit Plane Velocity Profiles for the 1.5 Inch Jet at the 25 and 65 Percent Chord Exit Locations. 
LIST OF ILLUSTRATIONS (CONTINUED)

Figure

Page

A-2. Free Jet Exit Plane Velocity Profiles for the 1.5 Inch Jet and the 3.0 Inch Jet at the 45 Percent Chord Exit Location . . . . . . . . . . . . . . .

A-3. Free Jet Exit Plane Velocity Profiles for the 1.5 Inch Jet at the 65 Percent Chord Exit Location Before and After Modification . . . . . . . . . 112

A-4. Free Jet Exit Plane Velocity Profiles for the 3.0 Inch Jet at the 25 Percent Chord Exit Location Before and After Modification . . . . . . . . . 113

A-5. Free Jet Decay Characteristics for the 1.5 Inch Jets . . . . . . . . . . . . . . . . . . . . . .

A-6. Free Jet Decay Characteristics for the Jets at the 45 Percent Chord Exit Location . . . . . . . . . . . . . 115

A-7. Free Jet Constant Velocity Core for the 1.5

Inch Jet at the 45 Percent Chord Exit Location . . . . . . . .

A-8. Free Jet Velocity Profiles for the 1.5 Inch

Jet at the 45 Percent Chord Exit Location 2.5

Inches from the Jet Exit Plane . . . . . . . . . . . . . .

B-1. Comparison of First and Second Balance

Calibrations for the Lift Component . . . . . . . . . . . . . . 128

B-2. Balance Calibrations for Drag and Drag Into Pitching Moment Interaction . . . . . . . . . . . . . . . . . 129

B-3. Balance Calibration for Pitching Moment. . . . . . . . . . 130

B 4. Comparison of Lift Calibration for Different Balance Bridging Arrangements . . . . . . . . . . . . . .

B-5. Balance Tares Resulting from System Pressurization . . . . . . . . . . . . . . . . . . . . . .

B-6. Schematic Diagram of Plenum Chamber Showing Calibration Axes and Location of Load Points. 


\section{LIST OF ILLUSTRATIONS (CONTINUED)}

Figure

Page

B-7. Internal Balance Normal Force Output in

Response to a Static Loading . . . . . . . . . . . . . .

B-8. Thrust as Measured on Internal Balanee

System Compared with Theoretical Values . . . . . . . . .

B-9a. Calibration of Pressure Measurement System

Against the Dead-Weight Tester . . . . . . . . . . . . . . .

B-9b. Calibration of Pressure Measurement System

Against the Dead-Weight Tester . . . . . . . . . . . . . .

B-9c. Calibration of Pressure Measurement System

Against the Dead-Weight Tester . . . . . . . . . . . . . . .

B-9d. Calibration of Pressure Measurement System

Against the Dead-Weight Tester . . . . . . . . . . . . . . .

B-10. Wind Tunnel Freestream Dynamic Pressure

Probe Arrangement . . . . . . . . . . . . . . . . . . . .

B-11. Results of Dynamic Pressure Survey of

Wind Tunnel Freestream . . . . . . . . . . . . . . .

B-12. Piezometer Setting for Desired Freestream

Dynamic Pressure . . . . . . . . . . . . . . . . . . .

C-1a. Interference Surface Pressure Distribution . . . . . . . . .

C-1b. Interference Surface Pressure Distribution . . . . . . . . . . 152

C-1c. Interference Surface Pressure Distribution . . . . . . . . . . . 153

C-1d. Interference Surface Pressure Distribution . . . . . . . . . . . 154

C-1e. Interference Surface Pressure Distribution . . . . . . . . . . 155

C-1f. Interference Surface Pressure Distribution . . . . . . . . . . 156

C-1g. Interference Surface Pressure Distribution . . . . . . . . . . 157 
LIST OF ILLUSTRATIONS (CONTINUED)

Figure

Page

C-1h. Interference Surface Pressure Distribution . . . . . . . . 158

C-1i. Interference Surface Pressure Distribution . . . . . . . . . 159

C-1j. Interference Surface Pressure Distribution . . . . . . . . 160

C-2a. Interference Surface Pressure Distribution . . . . . . . . 161

C-2b. Interference Surface Pressure Distribution . . . . . . . . . 162

C-2c. Interference Surface Pressure Distribution . . . . . . . . 163

C-2d. Interference Surface Pressure Distribution . . . . . . . . 164

C-2e. Interference Surface Pressure Distribution . . . . . . . . 165

C-2f. Interference Surface Pressure Distribution . . . . . . . . 166

C-2g. Interference Surface Pressure Distribution . . . . . . . . 167

C-2h. Interference Surface Pressure Distribution . . . . . . . 168

C-2i. Interference Surface Pressure Distribution . . . . . . . . 169

C-2j. Interference Surface Pressure Distribution . . . . . . . . 170

C-3a. Interference Surface Pressure Distribution . . . . . . . 171

C-3b. Interference Surface Pressure Distribution . . . . . . . . . 172

C-3c. Interference Surface Pressure Distribution . . . . . . . . 173

C-3d. Interference Surface Pressure Distribution . . . . . . . . . . 174

C-3e. Interference Surface Pressure Distribution . . . . . . . . . 175

C-3f. Interference Surface Pressure Distribution . . . . . . . . . 176

C-3g. Interference Surface Pressure Distribution . . . . . . . . . 177

C-3h. Interference Surface Pressure Distribution . . . . . . . . . 178 


\section{LIST OF ILLUSTRATIONS (CONTINUED)}

Figure

Page

C-3i. Interference Surface Pressure Distribution . . . . . . . . . 179

C-3j. Interference Surface Pressure Distribution . . . . . . . . . 180

C-4a. Interference Surface Pressure Distribution . . . . . . . . . . 181

C-4b. Interference Surface Pressure Distribution . . . . . . . . . 182

C-4c. Interference Surface Pressure Distribution . . . . . . . . . . 183

C-4d. Interference Surface Pressure Distribution . . . . . . . . . . 184

C-4e. Interference Surface Pressure Distribution . . . . . . . . 185

C-4f. Interference Surface Pressure Distribution . . . . . . . . 186

C-4g. Interference Surface Pressure Distribution . . . . . . . . . 187

C-4h. Interference Surface Pressure Distribution . . . . . . . . 188

C-4i. Interference Surface Pressure Distribution . . . . . . . . 189

C-4j. Interference Surface Pressure Distribution . . . . . . . . . 190

C-4k. Interference Surface Pressure Distribution . . . . . . . . 191

C-41. Interference Surface Pressure Distribution . . . . . . . . . . 192

C-4m. Interference Surface Pressure Distribution . . . . . . . . . . 193

C-4n. Interference Surface Pressure Distribution . . . . . . . . . . 194

C-4o. Interference Surface Pressure Distribution . . . . . . . . . . 195

C-4p. Interference Surface Pressure Distribution . . . . . . . . . . 196

C-4q. Interference Surface Pressure Distribution . . . . . . . . 197

C-4r. Interference Surface Pressure Distribution . . . . . . . . 198

C-4s. Interference Surface Pressure Distribution . . . . . . . . . . 199 


\section{LIST OF ILLUSTRATIONS (CONCLUDED)}

Figure

Page

C-4t. Interference Surface Pressure Distribution. . . . . . . . .

200

C-4u. Interference Surface Pressure Distribution. . . . . . . . . . . 201

C-4v. Interference Surface Pressure Distribution. . . . . . . . . 202

C-4w. Interference Surface Pressure Distribution. . . . . . . . . . 203

C-4x. Interference Surface Pressure Distribution. . . . . . . . . . . 204

C-4y. Interference Surface Pressure Distribution. . . . . . . . . . 205

C-4z. Interference Surface Pressure Distribution. . . . . . . . . 206

D-1a. Interference Lift Coefficients . . . . . . . . . . . . . . 210

D-1b. Interference Lift Coefficients . . . . . . . . . . . . 211

D-lc. Interference Lift Coefficients. . . . . . . . . . . . . . . 212

D-1d. Interference Lift Coefficients. . . . . . . . . . . . . 213

D-2a. Interference Drag Coefficients . . . . . . . . . . . . . 214

D-2b. Interference Drag Coefficients . . . . . . . . . . . . 215

D-2c. Interference Drag Coefficients . . . . . . . . . . . 216

D-2d. Interference Drag Coefficients . . . . . . . . . . . 217

D-3a. Interference Pitching Moment Coefficients . . . . . . . . . 218

D-3b. Interference Pitching Moment Coefficients . . . . . . . . . 219

D-3c. Interference Pitching Moment Coefficients . . . . . . . . . . 220

D-3d. Interference Pitching Moment Coefficients . . . . . . . . . . 221 
NOMENCLATURE
$\mathrm{A}_{j}$
- Jet Exit Area
c
- Wing Chord
$c^{\prime} \quad-$ Speed of Sound
$\mathrm{C}_{\mathrm{D}} \quad$ - Wing Drag Coefficient
$\mathrm{C}_{\mathrm{L}} \quad-$ Wing Lift Coefficient
$\mathrm{C}_{\mathrm{M}}$ - Wing Pitching Moment Coefficient with Respect to the Quarter Chord
$\mathrm{C}_{\mathrm{p}} \quad-$ Pressure Coefficient
$\overline{\mathrm{D}} \quad$ - Bellmouth Capture Diameter
$d_{j} \quad-\quad$ Jet Exit Diameter
$\mathrm{E}_{\mathrm{n}} \quad-$ Readout of Strain Gage Bridge $\mathrm{n}$
${ }_{\mathrm{N}}, \mathrm{F}_{\mathrm{R}}$, - Quantities Defined in Appendix B by:
${ }^{\mathrm{F}} \mathrm{P}$

$$
\begin{aligned}
& F_{N}=E_{1}+E_{2}+E_{3}+E_{4} \\
& F_{R}=\left(E_{1}+E_{2}\right)-\left(E_{3}+E_{4}\right) \\
& F_{P}=\left(E_{1}+E_{4}\right)-\left(E_{2}+E_{3}\right)
\end{aligned}
$$

h - Distance of Jet Exit Plane above Wind Tunnel Floor

$\mathrm{k}_{\text {min }} \quad-$ Minimum Bellmouth Clearance

L, D, PM - Total Wing Lift, Drag, and Pitching Moment, Respectively

N, R, P - Normal Force, Rolling Moment, and Pitching Moment Respectively Acting on Plenum Chamber 


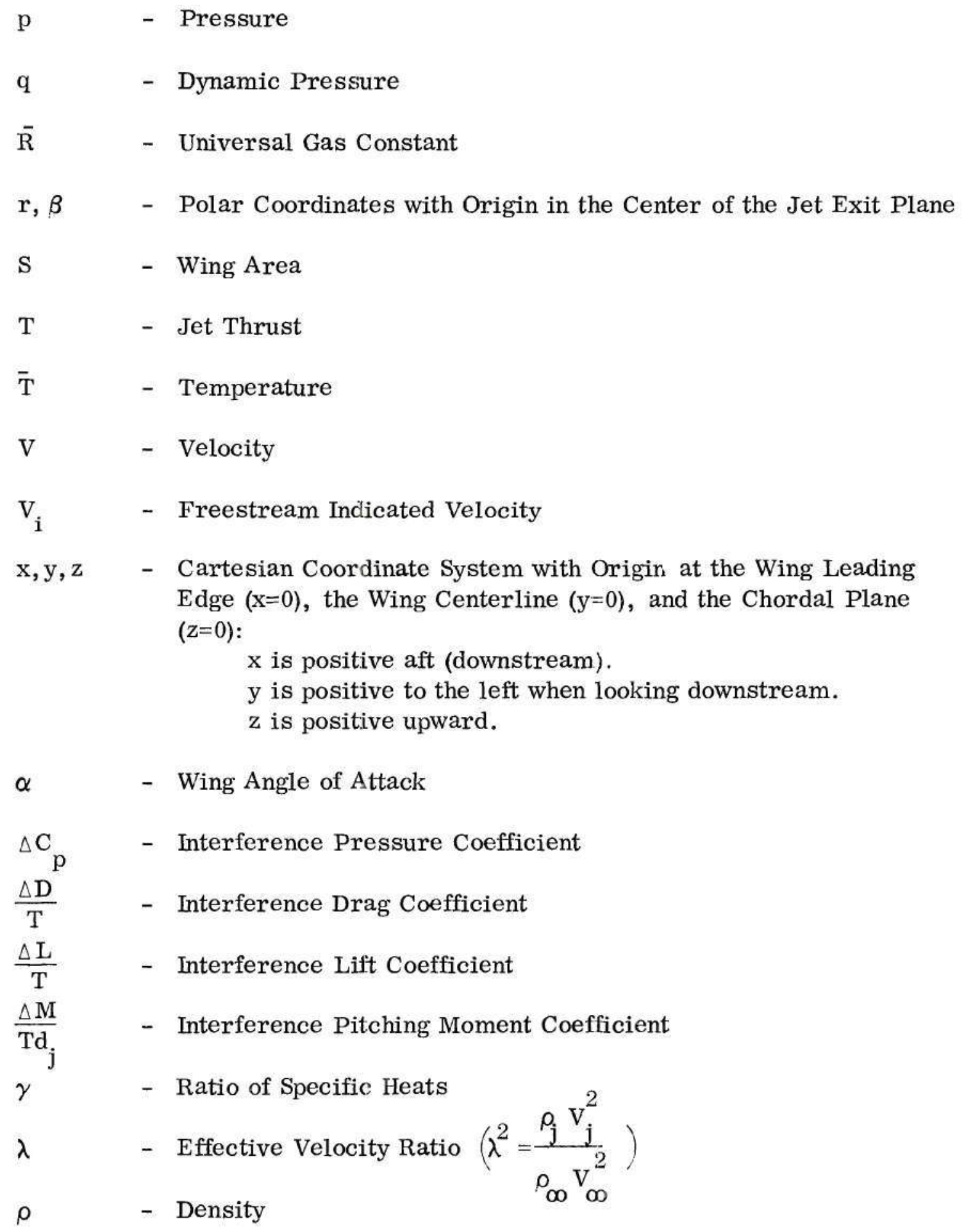

SUBSCRIPTS

a $\quad$ - Ambient 


$\begin{array}{ll}\text { B } & \text { - Wind Tunnel Balance Readout } \\ \text { C } & - \text { Wind Tunnel Balance Calibrated Value } \\ \text { c } & - \text { Corrected } \\ \text { j } & - \text { Jet Exit Plane } \\ \text { o } & - \text { Stagnation Conditions } \\ \text { p } & - \text { Plenum Chamber } \\ \text { s } & - \text { Local Static Value } \\ \text { T } & - \text { Wind Tunnel Balance Tare } \\ \text { z } & - \text { Above Jet Exit Plane (Appendix A) } \\ \infty & - \text { Freestream Conditions }\end{array}$




\section{SUMMARY}

The aerodynamic interference resulting from a jet issuing normal to the chordal plane of a two-dimensional wing in a crossflow has been experimentally investigated. The primary purpose of this work was to provide a link between previous investigations of a jet issuing into a crossflow from an infinite flat plate and the numerous experiments in which the aerodynamic characteristics of V/STOL aircraft configurations in transitional flight were determined.

This research was primarily conducted in the Georgia Tech nine-foot low speed wind tunnel utilizing an "infinite" span wing of NACA 0021 profile with a 15. 37 inch chord. A jet exit diameter of 1.5 inches with the jet centerline exit location at 25,45 , and 65 percent chord and a jet exit diameter of 3.0 inches exiting at 45 percent chord were the geometries tested. The ratio of jet exit plane velocity to freestream velocity (effective velocity ratio, $\lambda$ ) was varied in the range $2 \leq \lambda \leq 12$. Measurements were made principally at 0,6 , and 9 degree wing angles of attack. For each configuration, the surface pressure distribution was determined by surveying 190 surface pressure taps. These results are presented as contours of constant interference surface pressure for both the upper and lower wing surfaces. In addition, wing force and moment data were measured with the wind tunnel yoke balance and a direct measurement of the jet thrust was provided by a strain gage balance internal to the wing. These measurements determined the interference lift, drag, and pitching moment.

A comparison of the interference surface pressure contours of the wing 
lower surface with those of an infinite flat plate reveals that they are much the same for $\lambda>6$. The dissimilarity becomes greater as $\lambda$ is decreased primarily through the growth of an extensive region of positive interference surface pressure forward of the jet on the wing. Interference lift losses of approximately the same magnitude for all geometries were observed when $\lambda>6$. However, a lift augmentation occurred for $\lambda<6$ which was attenuated by increases in angle of attack, forward movement of the jet exit location, and decreasing jet exit size. The available data indicates that the character of the interference flow is distinctly different for high and low values of the effective velocity ratio. A comparison of the experimental interference pressure contours of the wing with those of the existing theory yielded only fair agreement. 


\section{CHAPTER I}

\section{INTRODUCTION}

There is currently a strong interest in airplanes that have vertical or very short takeoff and landing capability. Many airplanes of this type utilize a direct lift jet or lift fan to provide the required vertical thrust. However, it is a well established fact that such airplane configurations generally suffer a significant lift loss in the transition from hovering to forward flight. This is often accompanied by adverse changes in the pitching moment of the vehicle. These effects can be primarily attributed to the aerodynamic interference created by the interaction of the lifting jet efflux and the oncoming freestream with the adjacent lifting surface. This work is aimed at increasing the understanding of this phenomenon.

\section{Literature Review}

A large number of previous experimental investigations have been concerned with determining the aerodynamic characteristics of actual or proposed VTOL aircraft configurations. References 1 through 7 are typical examples in which a direct lifting jet (or jets) was considered and, in reference 8 through 14 , fans imbedded in the wings or fuselage were employed to provide the vertical thrust. Such investigations characteristically reveal lift loss and adverse pitching moment effects in transitional flight. Although these experiments were useful in determining the aerodynamic characteristics of the aircraft configurations under study, the models tested were so specific as to make the extraction of 
fundamental results or the delineation of general trends very difficult. Furthermore, several of these investigations were performed for a complete V/STOL airplane configuration thus possibly obscuring the main effects of the jet/lifting surface interaction (e.g., the interaction of the jet plume/vortex system and the model tail would affect any interference force or pitching moment measurements). The problem of a jet issuing from an infinite flat plate into a crossflow has also been widely studied. This configuration has merit since it is the simplest possible geometry that possesses the essential elements of the aerodynamic interference problem of interest. Several examples of this experimental work are found in references 15 through 20. A number of theoretical approaches to the flat plate problem, such as references 21 and 22 , have been able to produce creditably good agreement with the experiments. Hence, the problem of a jet effluxing from a flat plate can be considered well understood (with the exception of the wake region behind the jet) and at the least well documented.

The inherent justification for the flat plate work is that such results are directly extensible to the real case (a jet issuing from a lifting surface). If this is valid, the primary effect of the jet on a lifting surface would be to induce an effective downwash field. Knowing this distribution, then, would allow one to apply conventional lifting surface theory to obtain the desired aerodynamic characteristics. The possible shortcomings of this approach were pointed out by Williams and Wood. ${ }^{23}$ They reported the results of some preliminary experiments done at the Royal Aircraft Establishment for a jet issuing from a finite wing. A comparison was made for the interference surface pressure distribution between this finite wing 
and the flat plate at $\lambda=4$. The limited data available indicated large differences in the pressure contours between the wing and the flat plate (primarily in regions forward of the jet) that, in the opinion of the authors, could not be accounted for, even approximately, by appeal to conventional lifting surface theory.

It was also noted by Williams and Wood ${ }^{23}$ (and others, e.g. Reference 19) that the character of the interference flow field about the wing may be vastly different for high and low values of the ratio of jet velocity to crossflow velocity. They reported that the experimental results on a simple rectangular wing with a centrally located circular jet indicated, for $\lambda<4$, a reversal of the normally observed lift loss trends and an eventual lift augmentation as the velocity ratio was further decreased. This lift augmentation was accompanied by a substantial drag rise.

In a recent survey paper, Skifstad ${ }^{24}$ has commented on the need for reliable and complete experimental data for a limited range of flow conditions to aid in formulating theoretical models. He suggested that the degree of coupling between the aerodynamic field of the jet and that of finite airfoils be explored further experimentally. Skifstad also noted that regimes of strong and weak interaction conditions should be assessed.

The bridge between investigations dealing with actual airplane models and the infinite flat plate is the consideration of a jet issuing from a simple wing. This experimental configuration has not received nearly the attention given to aircraft configurations or the flat plate. The majority of the previous work using such an arrangement has been accomplished by imbedding a lifting fan in a finite wing. 
References 25 through 27 are typical of this experimental arrangement. A major shortcoming of all of these works is that adequate surface pressure measurements (necessary to obtain interference surface pressure contours) were not made. There are a number of other difficulties encountered when attempting to interpret the data from these investigations. Primary among these are the uncertain interference effects attributal to the fan inlet flow and also the question of the quality of the jet efflux. Furthermore, these configurations invariably have large fan diameters which results in uncertain wind tunnel wall interference--particularly at large values of the fan efflux to crossflow velocity ratio.

A recent attempt to overcome these difficulties was provided by Carter. ${ }^{28}$ In this investigation a finite rectangular wing was tested with and without flap deflection. The jet was supplied by an ejector system which was not an integral part of the wing model. This allowed for the jet exit location and the jet exit plane to be easily varied and also provided a convenient method for determining interference forces since forces on the wing and the jet thrust could be measured independently. However, the closest the jet exit plane could be positioned to the wing surface was one-half of the chordlength. The wing model used by Carter had an eight inch chord, a 48 inch span and included a four inch diameter mock fuselage on the wing centerline. The ejector units (one on each side of the fuselage) which supplied the jet efflux were 3.5 inches in diameter and 17.5 inches long. These units were supported separately from above by vertical struts located either just forward or aft of the wing depending on the desired jet exit location. Carter noted that his results were not the same (for the same jet exit plane and location) when 
the vertical struts passed aft of the wing as opposed to passing forward of the wing. Thus, the question of the mutual interference of the jet supply system and the wing must be considered since the size of the jet supply pipe was quite large compared to the size of the wing. A limited number of chordwise surface pressure measurements were made in this experiment.

Wooler, et al ${ }^{29}$ have reported on the results of a jet issuing from a finite wing of aspect ratio three. Air was supplied to the jet by a vertical pipe which passed through the wing upper surface and led to a centrally located jet $\left(d_{j} / c=0.08\right)$. This arrangement precluded direct measurements of the interference forces but chordwise pressure distributions on both surfaces were obtained at several span stations. Changes in the model angle of attack and jet exit geometry were not possible. This work also presents a theoretical model which showed good agreement with the limited data available. The comparison between experiment and theory is made for $\lambda=10$.

All of the experimental arrangements which have been discussed exhibited a number of shortcomings. In each instance, at least one of the following difficulties existed: a jet efflux of unknown quality, the possibility of significant mutual interference between the jet supply system and the wing model, and/or a limited flexibility in the choice of jet exit geometry. An experimental arrangement which would alleviate these difficulties is to consider a jet issuing from a simple two-dimensional wing with a jet air supply internal to the model as described below. 


\section{$\underline{\text { Research Objective and Scope }}$}

The experimental arrangement used in this investigation was a twodimensional wing installed in the Georgia Tech nine-foot low speed wind tunnel. A plenum chamber was located inside the model with jet air being supplied through hoses internal to the wing and entering both sides of the plenum chamber. Provisions were made for varying both the jet exit location and the jet exit diameter.

This configuration permitted the testing of simple jets whose characteristics in the absence of a crossflow were well defined. The plenum chamber was mounted on strain-gaged flexures, thus allowing a direct measurement of jet thrust. This enabled calculations of the interference lift, drag, and pitching moment to be made since the wind tunnel balance responded to total wing forces (including jet thrust). Furthermore, this arrangement provided a clean aerodynamic model which was free of mutual interference type errors. The model was instrumented with 190 surface pressure taps, thus allowing the interference surface pressure contours to be determined on both surfaces.

Interference force and pressure measurements have been made for values of $\mathrm{d}_{\mathrm{j}} / \mathrm{c}=0.10$ at $\mathrm{x}_{\mathrm{j}} / \mathrm{c}=0.25,0.45$, and 0.65 and for $\mathrm{d}_{\mathrm{j}} / \mathrm{c}=0.20$ at $\mathrm{x}_{\mathrm{j}} / \mathrm{c}=0.45$. Angles of attack included $0^{\circ}, 6^{\circ}, 9^{\circ}$, and, for the larger jet, $12^{\circ}$. The effective velocity ratio has been varied in the range $2 \leq \lambda \leq 12$.

The analysis of the data collected in the experiment has proceded with three primary goals.

1. A comparison of the interference surface pressures for the wing with those of the flat plate is made. The purpose, of course, is to determine if the 
abundance of flat plate data gathered by previous investigators is directly extensible to the lifting case.

2. The variation of the flow and geometry parameters in the investigation permits a direct comparison of the various cases for the purpose of establishing trends. Aspects of the interference flow which may be fundamentally different for high and low values of the effective velocity ratio are also identifiable.

3. A comparison with available theoretical calculations is made. This is used to show the applicability and limitations of the theoretical models.

The ultimate goal of research of this type is to be able to theoretically predict, in some sense, the aerodynamic characteristics of airplane configurations. To this end, the assemblage of data and the accompanying analysis should help to provide a more firm basis on which to assess future theoretical models. 
CHAPTER II

\section{EQUIPMENT AND INSTRUMENTATION}

The experimental configuration described in this chapter consists of a twodimensional wing equipped with a lifting jet. A description of the instrumentation required to measure aerodynamic forces and pressures is also given.

\section{Wind Tunnel}

The Georgia Tech nine-foot low speed wind tunnel is a closed return atmospheric type. The test section velocity is continuously variable with a maximum speed of approximately 225 feet per second. The tunnel turbulance factor is 1.2.

The tunnel is equipped with a six-component yoke balance which is of the completely automatic electro-mechanical type. Aerodynamic forces and moments are displayed on counters and the same data are printed by an eight-channel printer. For this investigation three channels were utilized to simultaneously record wing lift, drag, and pitching moment.

The balance system has a stated accuracy of 0.1 percent of the applied load for each component loaded separately, except for very small loads where accuracy is then limited by beam sensitivity. Component interactions are usually within \pm 0.1 percent of each individually applied load.

\section{Air Supply}

The jet air was supplied by a 100 h.p. centrifugal compressor. The flow 
was first led from the compressor into a six inch diameter pipe, then passed through an orifice plate and a butterfly valve which was used to control exit conditions, and finally passed to a $6 \times 2.5 \times 2.5$ inch (nominal) reducing tee. The compressor exit was equipped with a dump valve in order to avoid compressor surge (and subsequent flow oscillations) when a low mass flow was required. Compressor performance as stated by the manufacturer was a discharge pressure of five psi for an inlet air volume of 3700 cubic feet per minute and eight psi for 1000 cubic feet per minute.

\section{General Test Arrangement}

The two-dimensional model was mounted horizontally in the test section ten inches above the tunnel centerline. The general arrangement is shown in Figures 1 and 2. The model was supported at each end on external pylons which are an integral part of the platform balance, thus necessitating that extreme care be exercised to prevent grounding of the balance system. This two-dimensional horizontal orientation of the model was chosen because it allowed the model to be essentially free of any mutual interference effects that would have resulted had the model been mounted on a fork support inside the tunnel for example. Another possibility was to cantilever the model vertically from the tunnel floor but this was discarded because the desired jet mass flow required that air be supplied through both ends of the model. This requirement would have made a satisfactory balance bridge difficult to attain. (Ideally, to obtain accurate balance readings, the model should be supported only on the balance system and be completely free of any connections that might ground or foul the system. The necessity of supplying jet air to a model 
such as this and of routing electrical leads and pressure lines off the model make the ideal situation impossible. The arrangement which was used to minimize interference with the balance is collectively referred to as the balance bridge in this dissertation.)

The modification required to the test section to insure two-dimensionality is shown in Figure 2 and Figure 3. This consisted of constructing flat sidewalls in the wind tunnel test section and providing an interface at the wing/sidewall junction. The sidewalls had a maximum displacement of eleven inches from the circular wind tunnel walls thus yielding a model span of 86 inches. These sidewalls extended approximately seven feet upstream into the wind tunnel converging section and in this region were designed to maintain a vertical plane up to the point of fairing with the tunnel wall. A calibration of the tunnel freestream dynamic pressure with this modification indicated a spanwise uniformity within one percent of the centerline $q_{\infty}$ (See Appendix B). A maximum freestream velocity of $150 \mathrm{ft} / \mathrm{sec}$ was possible with the sidewalls in place.

Behind the wing endplate pictured in Figure 3 there was an annular channel mounted on the flat sidewall. Initially, a bicycle inner tube served as an inflatable seal between the metric endplate (fixed to the wing) and the sidewall. However, it was discovered that neither force nor pressure measurements were affected by the inflation or deflation of this seal. Furthermore, it was found that this seal was fouling the wind tunnel balance bridge even when deflated (See Appendix B) and hence it was removed with no noticeable effect on either force or pressure measurements. The jet air supply to the model was provided by a pair of 2.5 inch inside 
diameter by 50 foot lightweight vacuum hoses (Gates Model $770 \mathrm{SB}$ ) with enlarged ends to provide smooth flow and with built-in nipples for connection to the swivel joints at the end of the supply pipe into wing. The hose on the tunnel south side passed through the floor of the operator's work area and was hung in a large loop before mating with the tee. On the north side, a balance side force link prevented a similar arrangement. Hence the hose was angled approximately $45^{\circ}$ in the upstream direction and was again hung in a large loop before attachment to the tee. This arrangement provided balance zero returns within $\pm 0.2 \mathrm{lbs}$ (or ft. $-\mathrm{lbs}$.). (Two separate calibrations were made of the wind tunnel balance system as described in Appendix B. The stated zero return figure was for the second calibration.) Provisions were made for a manual adjustment of the model angle of attack. The model support on each balance pylon consisted of an air supply pipe passing through a pillow block (Dodge Model SCM). An adjustable friction sleeve on the pylon caps allowed the model to be rigidly locked in rotation. An incidence alignment bar mounted on the supply pipe had close tolerance holes which aligned with those of an incidence plate rigidly mounted to the pylon cap thus providing the means for setting the angle of attack. Swivel joints (T-M Model No. 200) were affixed to the outboard ends of the supply pipe. Before the second balance calibration, these joints were rigidly attached to the balance pylon caps (See Appendix B) to assure that the flexible supply hoses would always hang in the same position with varying angles of attack. A static balance calibration at different angles of attack yielded identical results. 


\section{Two-Dimensional Model}

The profile of the two-dimensional model was a modification of the NACA 0021 airfoil. The coordinates of the profile used are given in Table 1. The modification consisted of adjusting the coordinates from 80 percent chord to the trailing edge such that a straight contour resulted. This was done to facilitate construction of the model (Table 1 reveals that this change yielded a 15.37 inch chord. However, for descriptive purposes in this dissertation a 15 inch chord is used. For example, the 1.5 inch diameter jet is said to be $d_{j} / c=0.10$. Of course, all data reduction calculations used the actual length of the chord when appropriate.) This particular symmetrical airfoil profile was chosen partly to simplify model construction and also because the pressure gradient on both surfaces was essentially constant for a significant fraction of the chord (See Chapter IV). The maximum thickness of 21 percent chord was necessary to allow adequate space for the internal plenum chamber, the associated air supply, and the surface pressure lines.

The model design incorporated steel front and rear spars with aluminum plate ribs appropriately spaced along the span. An assembly drawing of the wing is presented in Figure 4. The wing ribs were manufactured to a tolerance of \pm 0.002 inches utilizing a numerically controlled milling machine. The same tolerance was maintained on the solid aluminum leading and trailing edge sections. Skin panels consisted of 0.063 inch aluminum sheet and were rolled to the approximate rib contour prior to installation. Figure 5 shows the assembled wing with the skin panels removed and Figure 6 shows a wing panel with surface pressure lines installed.

Early in the experimental program, it was decided that an artificially 
Table 1. Coordinates of the Modified NACA 0021 Profile

(Symmetrical)

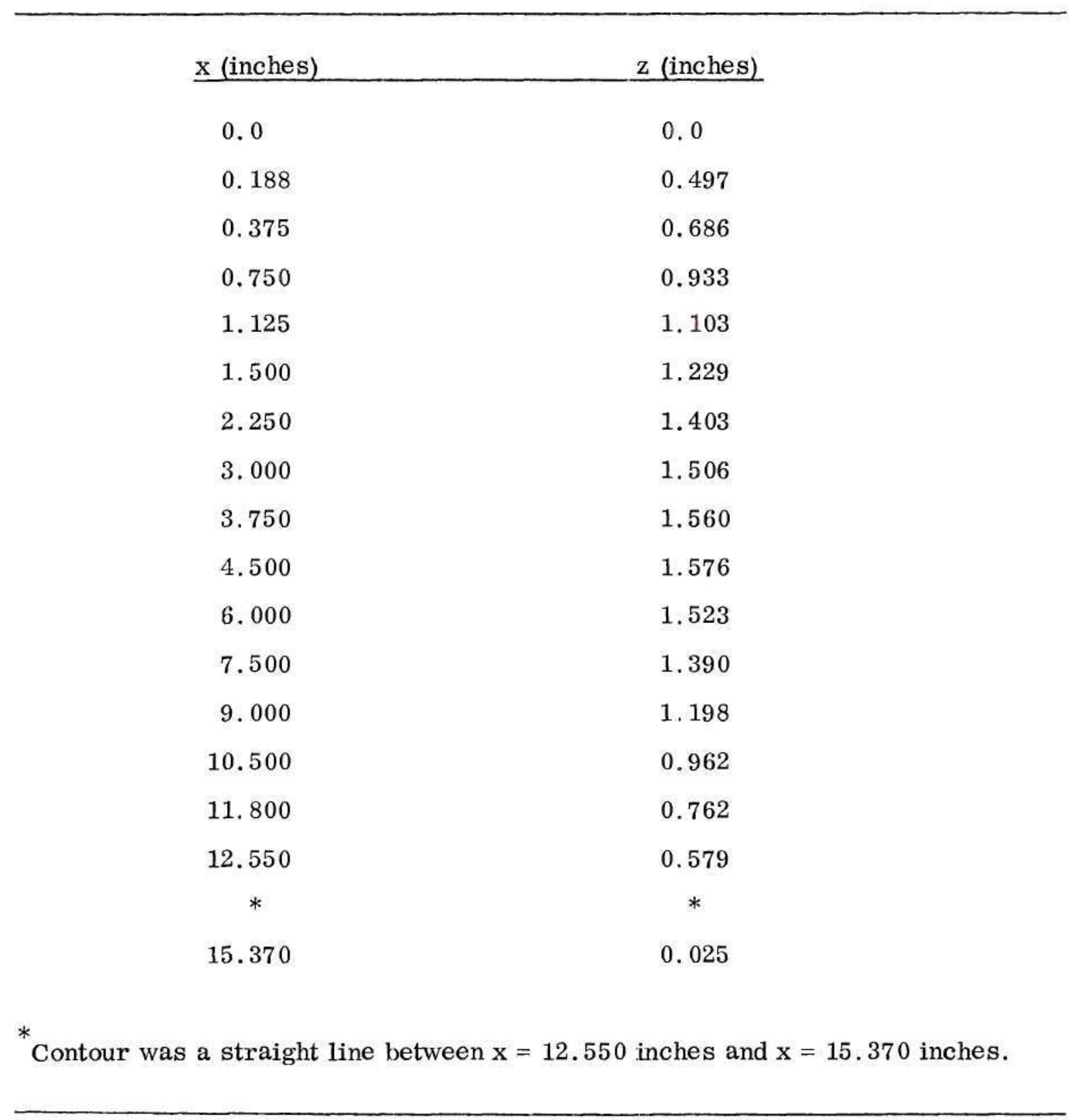


induced layer transition was required (see Chapter IV). This was accomplished by spraying 0.096 inch diameter glass spheres on a portion of the wing surface which had been covered with an epcxy resin mixture. These transition strips (on both surfaces) were approximately 0.38 inches wide, were centered at about five percent chord, and covered the model span. All panel joints and countersunk screw heads were covered with cellophane tape thus making for a smooth surface for the remainder of the wing.

\section{Plenum Chamber and Jet Nozzles}

The plenum chamber was designed to be positioned inside the wing in such a fashion that it would be isolated from the structure and all internal instrumentation. The chamber was held in place by four strain-gaged flexures, thus allowing a direct measurement of jet thrust. An assembly drawing of the plenum chamber is shown in Figure 7 .

The jet air supply from the 2.5 inch pipes which passed through the pillow blocks on each side emptied into external plenum chambers which were located just outboard of the wind tunnel sidewalls (See Figure 1 and Figure 4). Three 1.5 inch diameter flexible hoses (Gates Model $770 \mathrm{SB}$ ) then led from each external plenum chamber into the wing. These hoses mated with the internal plenum chamber through flexible stainless steel bellows (Cajon Company, Model No. X-24-3) as shown in Figure 8. The use of these bellows permitted the plenum chamber to "float" on the flexures (see following section on force measurement system) that were instrumented with strain gages for the jet thrust measurements. Note also in Figure 8 the six frames inside the plenum chamber which supported fine mesh screens which 
facilitated achieving uniform flow conditions at the jet exit plane.

The design of the plenum chamber provided for testing a 1.5 inch diameter jet at values of $x_{j} / c$ of $0.25,0.45$, or 0.65 . In addition, a 3.0 inch diameter jet could be tested at $x_{j} / c$ values of 0.25 and 0.45 . The jet nozzles consisted of a simple bellmouth with a straight extension (typically, a nominal length of 0.32 inches) from the plenum chamber to the model surface. To obtain a uniform jet exit flow for such a nozzle, Peake ${ }^{20}$ has suggested a minimum bellmouth clearance, $\mathrm{k}_{\text {min }}$, inside the plenum chamber given by $\mathrm{k}_{\min }=1.11 \mathrm{~d}_{\mathrm{j}}^{2} / \overline{\mathrm{D}}$ where $\overline{\mathrm{D}}$ is the bellmouth capture diameter. For the 1.5 inch jet used here, $\mathrm{k}_{\min }=0.83$ inches and for the 3.0 inch jet, $\mathrm{k}_{\min }=2.22$ inches. An actual clearance of 1.00 inches at $\mathrm{x}_{\mathrm{j}} / \mathrm{c}=0.25$ and 0.45 for both jet sizes and an actual clearance of 0.20 inches for $\mathrm{x}_{\mathrm{j}} / \mathrm{c}=0.65$ existed. Hence, the criteria was met only by the 1.5 inch jet at the two forward locations. An investigation of the jet exit flow in free air revealed that these two jet configurations displayed uniform exit plane velocity profiles. Considerable experimentation with screens and the internal plenum geometry (See Appendix A) was required before the other jets exhibited similar characteristics. A photograph of some typical jet nozzle assemblies is presented in Figure 9. (The nozzle assembly in the lower left hand corner of Figure 9 has a 0.75 inch jet exit diameter and was not used in the experiment.)

A drawing of the installation of the plenum chamber in the wing is shown in Figure 10. The actual installation is presented in Figure 11 in which the removable plate assembly containing the nozzle can be easily seen.

For each nozzle, the straight exit extension was fashioned to be flush with 
the wing surface contour. Since the thrust was determined by measuring the reacting force on the plenum chamber, it was necessary to provide a seal at the nozzle/wing panel interface. Dental dam held in place with General Electric RTV108 silicone adhesive was used for this purpose. Figure 12 is a photograph of the model ready for testing with this seal in place. Since all wing joints were taped and the plenum chamber was tested to be leak-tight, internal flow in the wing or accidental differential pressures across the plenum chamber were minimized.

\section{Pressure Measurement Instrumentation}

The wind tunnel freestream dynamic pressure as determined by measurements using piezometer rings was monitored by the tunnel operator on a Betz water manometer (reading uncertainty of $\pm 0.03 \mathrm{~mm} \mathrm{H}_{2} \mathrm{O}$ ). A calibration of the Betz system and a $\mathrm{q}_{\infty}$ survey is presented in Appendix B. In addition, a pitot static probe was mounted on the tunnel ceiling (See Figure 2) and the dynamic pressure was then displayed on a strip chart recorder. Free stream stagnation temperature was measured using a bulb type aircraft thermometer and ambient atmospheric pressure by using an anereoid barometer.

The plenum chamber stagnation pressure was monitored with a Barocel Electronic Manometer (CGS, Datametrics Division, Type 1014) in conjunction with a 7.5 psi variable capacitance pressure transducer (CGS, Datametrics Division, Type 511). Output was displayed on a digital voltmeter. The stagnation pressure port was located on the geometric center of the forward bulkhead of the plenum chamber. A chromel-alumel thermocouple was installed just beneath this port for monitoring plenum stagnation temperature. Thermocouple output was measured on 
a self-compensating millivolt potentiometer (Leeds and Northrup Co., Cat. No. 8686) with ambient temperature (measured on an adjacent mercury thermometer) serving as the reference junction.

The wing was instrumented with 190 surface pressure taps. The location of the taps is shown in Figure 13. The pressure taps were so located as to provide an increase in their density close to the jet exit. This was done in anticipation of large pressure gradients (for jet operation) in the immediate vicinity of the jet. Of course, the primary factor in the tap locations was to insure that adequate interference pressure contours could be obtained. The spanwise extent was estimated from examination of existing flat plate data. Pressure taps in the wing panels were installed by inserting $1 / 16$ inch diameter (1/32 inch inside diameter) stainless steel tubes, which had been preformed with a $90^{\circ}$ bend, into interference fit holes in the panels and then applying Hy-Sol epoxy patch to the joint. The protruding end of the tube was filed and then sanded flush with the wing surface. The flexible plastic tube pressure lines (3/32 inch outside diameter, manufactured by Borden) were connected to the stainless steel taps and then (in the center wing region) bonded to the skin panel with General Electric silicone adhesive. The bonding was necessary to insure that the pressure lines would not foul the plenum chamber and interfere with the thrust measurements. A typical instrumented center panel is shown in Figure 6. The pressure taps in the leading and trailing edges were similarly installed except that the stainless steel tubes did not protrude to the model surface and the lines were routed through the machined channels shown in Figure 13.

The 48 pressure leads in the panel which contained the jet exit were connected 
to a scanivalve (Mode1 48J4) which was mounted on that panel. Each jet exit configuration required an instrumented panel which would accept this scanivalve. (The center panel used for the jet-off testing is shown in Figure 6.) This arrangement allowed the making of model changes with minimum time and effort. The remainder of the pressure lines were routed out of the wing (see Figure 5) to be connected with scanivalves mounted on the two external plenum chambers. A total of 96 lines went to the double scanivalve (Model $48 \mathrm{~J} 4 \mathrm{GM}$ ) on the north end and the remaining 46 lines to the single scanivalve at the south end which is pictured in Figure 14. A pressure line from the total pressure line of the freestream pitot static tube and a line connected to a static pressure tap located on the wind tunnel sidewall in the plane of the chord approximately 15 inches forward of the model leading edge were also connected to this south scanivalve. This enabled an automated recording of $\mathrm{q}_{\infty}$ for each run. This arrangement resulted in a total of ter pressure lines (plus a thermocouple wire and eight strain gage electrical leads) which had to bridge the balance system. This was successfully accomplished by hanging all lines and leads in loops that were as large as possible without fouling on the wind tunnel walls.

Pressure measurements from the scanivalves were made utilizing a HewlettPackard Automated Data Acquisition System consisting of the Model 2114B Computer and the Model 2401C Integrating Digital Voltmeter. Scanivalve stepping was accomplished through a five channel solenoid controller (Scanivalve Corp., Model CTLR2/ $5 x$ S4) in conjunction with the computer. The output pressure lines of the scanivalves were connected to four $1000 \mathrm{~mm}$ Barocel Pressure Transducers (CGS, Datametrics Division, Type 501). The transducer output for each channel was interfaced with the 
digital voltmeter of the computer by signal conditioners (CGS, Datametrics Division, Type 1015-S2). The calibration of this pressure measurement system is discussed in Appendix B. Figure 15 is a photograph of the operator's work area in which the signal conditioners, solenoid controller, computer teletype and the electronic manometer for monitoring plenum stagnation pressure can be seen.

Force Measurement System

The existing wind tunnel balance system was used to measure the total lift, drag, and pitching moment acting on the model.

The desirability of making direct thrust measurements necessitated isolating the plenum chamber inside the wing. The plenum chamber was supported by a flexure at each corner which was mounted on a "floating" rib. These ribs did not make contact with the bellows or skin and were free to rotate and to slide horizontally in slots in both the front and rear spars. The rotational freedom resulted in the flexure behaving as if it were simply supported at the rib end (increasing the bridge output for a given load) and the horizontal freedom allowed for thermal expansion. Gross horizontal movement of the plenum chamber was restricted by the flexible stainless steel bellows. This arrangement is shown in Figure 16 . The flexures had an effective length of 1.00 inches, were 0.075 inches thick, and were machined from aluminum bar stock. On the top and bottom of each flexure a double strain gage (Micro-Measurements, Type EA-13-125MK-120) was mounted approximately 0.25 inches from the built-in end at the plenum chamber with Eastman 910 cement. These gages had a $120 \pm .24 \mathrm{ohm}$ resistance, a gage factor of $2.105 \pm$ . 011, and were self-temperature-compensating. Each flexure was wired as a 
standard four-arm bridge. The installed bridge can be seen in Figure 17. To eliminate the inherent mechanical hysteresis of the strain gage balance system (possibly caused by friction where the ribs were mounted by pins riding in the slots in the spars), a 60 -cycle vibrator was installed under the south external plenum chamber. This device was activated whenever wind tunnel balance or internal balance measurements were being made.

The thrust measurements were also made using the Hewlett-Packard computer and integrating digital voltmeter. Strain gage bridge channel selection was controlled through a Monsanto Data Scanner (Model 508A). The bridge input voltage was provided by a Hewlett-Packard Power Supply (Model 6261A) and was kept at a constant value of $5.00 \pm .01$ volts by monitoring with a digital voltmeter.

The calibrations used to determine the characteristics of both the wind tunnel balance and the internal balance are presented in Appendix B.

\section{Flow Visualization}

The flow visualization techniques used in this experiment were wool tufts and oil flow. The tufts consisted of two to three inch segments of sport yarn which were attached to the surface under study with cellophane tape.

For the oil pattern visualization the following mixture (by volume) was used:

$\begin{array}{lr}\text { Diesel Fuel No. } 1 & 16 \text { parts } \\ \text { Carbon Black } & 4 \text { parts } \\ \text { Oleic Acid } & 4 \text { parts } \\ \text { Linseed Oil } & 1 \text { part }\end{array}$

The wing was covered with a white self-adhesive plastic to prevent clogging of the surface pressure taps. This plastic covered the entire span from just afi of the 
transition strip to the trailing edge. For each run, a uniform coating of the above mixture was applied to the wing surface. The flow pattern required from three to ten minutes to develop depending on the freestream velocity. The developed oil pattern was then photographed. 


\section{CHAPTER III}

\section{TESTING PROCEDURE AND ACCURACY}

The technique used for setting the freestream velocity and the jet velocity for a particular velocity ratio, $\lambda$, is describec in this chapter. This procedure was the same for surface pressure or force measurement runs. The data reduction procedure and details of acquiring the surface pressures and the forces are also presented.

\section{Setting of Test Conditions}

The wind tunnel freestream velocity was set by the wind tunnel operator on a Betz water manometer at the desired indicated velocity, $\mathrm{V}_{\mathrm{i}}$. The setting was determined by the following equation.

$$
\text { Betz Reading (in. of } \mathrm{H}_{2} \mathrm{O} \text { ) }=.01149 \mathrm{v}_{\mathrm{i}}^{2}-.118
$$

This equation was determined by the calibration described in Appendix B. This procedure corresponds to using consistent values of the freestream dynamic pressure, i.e.

$$
q_{\infty}=\frac{1}{2} \rho v_{i}^{2}
$$

where

$$
\rho=.002378 \frac{\text { lbs. }-\mathrm{sec}^{2}}{\mathrm{ft}^{4}}
$$


That is, $V_{i}$ is the velocity which would yield the set value of $q_{\infty}$ under standard sea level conditions of temperature and pressure. The standard indicated velocity used for each $\lambda$ was that given in Table 2 . The freestream velocities used in the experiment and presented in 'Table 2 are a result of an effort to always run at as high a jet velocity as possible. This enabled more accurate force measurements to be made. Generally lower freestream velocities were used for the larger jet $\left(d_{j} / c=0.20\right)$ for corresponding values of $\lambda$ because of the smaller discharge pressure available from the compressor at the largex mass flow rates.

Once the tunnel was brought up to speed, a direct measurement of $\mathrm{q}_{\infty}$ utilizing the automated data acquisition system was made. Recall from Chapter II that one scanivalve port was connected to a freestream total pressure probe and another to a freestream static pressure tap. Since the reference pressure for all Barocel transducers was the ambient atmospheric pressure, the following equation determined $\mathrm{q}_{\infty}$ :

$$
q_{\infty}=\left(p_{0 \infty}-p_{a}\right)-\left(p_{\infty}-p_{a}\right)
$$

Then, the computer calculated the desired value of $q_{j}$ by

$$
q_{j}=\lambda^{2} q_{\infty}
$$

and proceeded to compute and display the digital voltmeter setting which would yield the appropriate plenum chamber stagnation pressure from previous calibrations (See Appendix B). A distinct advantage to this procedure was the perfunctory 
Table 2. Standard Freestream Indicated Velocities Used for Each $\lambda$

\begin{tabular}{ccc}
\hline$\lambda$ & $\begin{array}{c}\mathrm{V}_{\mathrm{i}} \text { (ft/sec) } \\
\mathrm{d}_{\mathrm{j}} / \mathrm{c}=0.10\end{array}$ & $\begin{array}{c}\mathrm{V}_{\mathrm{i}}(\mathrm{ft} / \mathrm{sec}) \\
\mathrm{d}_{\mathrm{j}} / \mathrm{c}=0.20\end{array}$ \\
\hline 12 & 60.5 & 32.8 \\
10 & 70.0 & 39.4 \\
8 & 100.0 & 49.2 \\
6 & 100.0 & 65.7 \\
5.9 & $*$ & 60.5 \\
4 & 150.0 & 98.4 \\
3 & 150.0 & 131.4 \\
2.5 & 150.0 & 150.0 \\
2 & 150.0 & 150.0 \\
\hline No data taken for this configuration & \\
\hline
\end{tabular}


check provided the electronic pressure measurement system prior to each run.

The freestream velocity was constantly monitored by the wind tunnel operator and could be held to within \pm 0.5 percent of setting. The jet plenum pressure was also constantly monitored. Adjustments to the mass flow rate were usually required until the plenum chamber stagnation temperature stabilized at about $160^{\circ} \mathrm{F}$. The plenum pressure was maintained within \pm 1.0 percent of reading.

No wind tunnel wall corrections were made in the present work. Tyler and Williamson ${ }^{30}$ have suggested that the parameter $\lambda \frac{d_{j}}{h}$ where $h$ is the distance from the jet exit plane to the tunnel wall (about 60 inches in this experiment) can serve as an appropriate wall effect criterion. They state that for a jet inclined $15^{\circ}$ to the flow (corresponding to $\alpha=15^{\circ}$ in the present work), a value of $\lambda \frac{d_{j}}{h} \leq 1.2$ is required to prevent separation of the freestream flow on the tunnel walls forward of the model. For the model in the present experiment at $\lambda=12$ and with $d_{j} / c=0.20, \lambda \frac{d_{j}}{h}=0.6$. Since Tyler and Williamson considered their criterion to be conservative with respect to the onset of adverse wall interference, it was assumed on the basis of the above calculations that adverse wall effects would not be a significant factor in this experiment.

\section{Surface Pressures}

All surface pressures were acquired with the Hewlett-Packard Automated Data Acquisition System described in Chapter II. The measurements acquired were converted by the computer such that the printed output consisted of $\mathrm{p}_{\mathrm{s}}-\mathrm{p}_{\mathrm{a}}$ in $\mathrm{mm}$ of $\mathrm{H}_{\mathrm{g}}$. The conversion to coefficient form then utilized the equation 


$$
c_{p}=\frac{\left(p_{s}-p_{a}\right)-\left(p_{\infty}-p_{a}\right)}{q_{\infty}}
$$

where the values for $q_{\infty}$ and $p_{\infty}$ recorded during the pressure run were used. A flow chart describing the basic functions of the data acquisition system for pressures is presented in Figure 18.

The first wind tunnel runs were used to determine the jet-off pressure distributions. The characteristics of this airfoil without jet operation are discussed in Chapter IV. All of the jet-off pressure coefficients used in the determination of interference pressure coefficients were taken with $V_{i}=100 \mathrm{ft} / \mathrm{sec}$. Subsequent runs were then made to obtain the interference pressure coefficients which were presented as

$$
\Delta \mathrm{C}_{\mathrm{p}}=\mathrm{C}_{\mathrm{p} \text { jet-on }}-\mathrm{C}_{\mathrm{p} \text { jet-off }}
$$

A summary of the interference pressure distributions is contained in Appendix C.

The calibration of the pressure measurement system (Appendix B) revealed that the pressure outputs were within \pm 1.0 percent of the applied pressure provided by the dead-weight tester. However, the observed fluctuations of the pressure readings during a run in some cases were considerably larger than this figure. For example, maximum deviations of about \pm 2.0 percent of $q_{\infty}$ in regions forward of and lateral to the jet and of \pm 5.0 percent of $q_{\infty}$ in the wake region aft of the jet were observed. These fluctuations agree with those noted by Mosher ${ }^{15}$ in a flat plate investigation. To minimize the effect of the fluctuations, each pressure 
measurement was recorded ten times and then a simple average computed. The integrating digital voltmeter was set on a period of 0.1 seconds for each measurement. Since the signal conditioners were autoranging, each measurement required a pressure output signal reading and a scale factor reading. This resulted in approximately 40 minutes being required for each pressure run of 190 readings.

Data reduction was accomplished through the facilities of the Rich Electronic Computer Center using the Univac 1108. For each run, the Hewlett-Packard Computer punched a paper tape containing the appropriate data. This data was transmitted to the Univac 1108 through a remote Demand Mode terminal. The primary functions of the data reduction program were to present the pressures in coefficient form, to organize the data, and to prepare a card deck containing the reduced pressure data. This deck then served as the input for a software routine prepared by California Computer Products, Inc. (General Purpose Contour Program). This routine used rather sophisticated mathematical techniques to develop the pressure contours which were then machine plotted. Further details of the data reduction are included in Appendix C.

The repeatability of the surface pressure measurements with the jet-on was within \pm 2.0 percent of $\mathrm{C}_{\mathrm{p}}$ in the regions forward of and lateral to the jet. In the fluctuating wake region aft of the jet, the pressure measurements were repeatable to within \pm 4.0 percent of $\mathrm{C}_{\mathrm{p}}$. Figure 19 is a comparison of the same flow/ geometry conditions for different runs and is typical of the results for the repeatability checks that were made. Also of concern is the dependence of the interference pressure contours on tunnel velocity. A comparison of the data for the same case 
which was run at two different tunnel speeds is made in Figure 20. The results indicate differences that are of the same order as the repeatability. The effect of varying freestream velocity on surface pressure measurements is discussed further in Chapter IV.

\section{$\underline{\text { Force Measurements }}$}

The Hewlett-Packard system was also used to record the force measurements. This was done primarily to assure a uniform technique for recording the strain gage outputs of the internal balance. A flow chart of the automated procedure for acquiring the force data is shown in Figure 21.

The interference lift, drag, and pitching moment is presented in the following form.

$$
\begin{aligned}
& \frac{\Delta L}{T}=\frac{L_{\text {jet-on }}{ }^{-L_{\text {jet-off }}}}{T} \\
& \frac{\Delta D}{T}=\frac{D_{\text {jet-on }}-D_{\text {jet-off }}}{T} \\
& \frac{\Delta M}{T d_{j}}=\frac{P_{\text {jet-on }}-P M_{\text {jet-off }}}{T d_{j}}
\end{aligned}
$$

Note that when the data is presented in this form that zero interference lift corresponds to $\frac{\Delta \mathrm{L}}{\mathrm{T}}=1.0$ (i.e. for a lift loss, $\frac{\Delta \mathrm{L}}{\mathrm{T}}<1.0$ ). Conversely, zero interference drag yields $\frac{\Delta D}{T}=0.0$ and zero interference pitching moment results in $\frac{\Delta \mathrm{M}}{\mathrm{Td}_{\mathrm{j}}}=0.0$. These equations can be modified to account for angle of attack since the jet exit plane is always the same as the geometric chord. The corrected 
equations can be written as

$$
\begin{aligned}
& \left.\frac{\Delta L}{T}\right)_{c}=\frac{L_{\text {jet-on }}{ }^{-L_{\text {jet-off }}}}{T}+(1-\cos \alpha) \\
& \left.\frac{\Delta D}{T}\right)_{c}=\frac{D_{\text {jet-on }}-D_{\text {jet-off }}}{T}-\sin \alpha
\end{aligned}
$$

The interference moment can be corrected by accounting for the fact that the thrust is not always acting at the quarter chord.

$$
\left.\frac{\Delta M}{T d_{j}}\right)=\frac{P M_{j e t-o n}-P M_{j e t-o f f}}{T d_{j}}+\frac{\left(x_{j} / c-.25\right)}{d_{j} / c}
$$

The uncorrected equations were chosen for data presentation because the adverse effect of the jet thrust at angle of attack is included (as the angle of attack is increased, a component of the jet thrust gives a positive drag since the thrust always acts normal to the wing chordal plane) and the favorable effect of a jet exit location aft of $\mathrm{x}_{\mathrm{j}} / \mathrm{c}=0.25$ is also included (all pitching moment data are presented with respect to the quarter chord and hence a positive thrust aft of this point results in a favorable pitching moment effect). Thus, the uncorrected data provides a more realistic assessment of a practical lifting jet installation. The interference lift coefficient is quite nearly identical in both cases.

As discussed in Chapter IV, the jet-off aerodynamic coefficients were found to be a function of tunnel speed. The data reduction, again utilizing the Univac 1108, made appropriate corrections for this variation in the calculations of the 
interference coefficients by using the jet-off aerodynamic coefficients that corresponded to the $q_{\infty}$ at which the jet-on measurements were taken. The details of these calculations and a summary of the interference force data collected are contained in Appendix D.

The jet thrust calculations were made using the procedure outlined in Appendix B. Each reading of the individual strain gage bridge outputs was made ten times and a simple average computed. For the thrust measurements, the period of the integrating digital voltmeter was set at 1.0 seconds. The accuracy of the measurement was estimated to be within \pm 2.0 percent of the actual thrust except at thrust levels lower than about four pounds. For a thrust of $2.5 \mathrm{lbs}$, the accuracy of measurement deteriorated to about \pm 10 percent of the actual thrust. This was largely attributed to the effect the dental dam seal had on the internal balance system for the smaller applied loads. This conclusion is based on the several static calibrations performed on the internal balance with loads of less than five pounds where errors of less than \pm 1.0 percent of the applied loads were noted. The only element missing from the actual test arrangement for these calibrations was the dental dam seal. In this experiment, the thrust varied in the range $2.0 \leq \mathrm{T} \leq 20.0$ pounds. No attempt was made to compute theoretical thrust levels under crossflow conditions because of the difficulty in determining the jet exit static pressure and the actual jet mass flow rate.

However, the major source of error in the interference force measurements came from the wind tunnel balance system. For example, the balance lift readings were considered accurate to within $\pm 0.2 \mathrm{lbs}$. as stated in Chapter $\Pi$. This results 
in an error in $\Delta \mathrm{L} / \mathrm{T}$ (for $\mathrm{d}_{\mathrm{j}} / \mathrm{c}=0.10$ ) of \pm 4 percent at $\lambda=8, \pm 10$ percent at $\lambda=4$, and about \pm 20 percent at $\lambda=2$. This deterioration in accuracy with decreasing $\lambda$ is a direct result of the corresponding decrease in the magnitude of the thrust. That is, for $\lambda=2$ and $\mathrm{d}_{j} / \mathrm{c}=0.10$, the measured jet thrust is only about 2.5 Ibs. Thus, the largest error arises when the interference lift with its accuracy of $\pm 0.4 \mathrm{lbs}$ for $\mathrm{L}_{\text {jet-on }}-\mathrm{L}_{\text {jet-off }}$ is divided by the thrust which also has a moderate inaccuracy as previously noted. Nevertheless, for the purpose of establishing trends, this level of accuracy was deemed acceptable. For the larger jet $\left(\mathrm{d}_{\mathrm{j}} / \mathrm{c}=\right.$ 0.20 ), the error in $\Delta \mathrm{L} / \mathrm{T}$ is within \pm 5.0 percent for all $\lambda^{\prime}$ 's because of the correspondingly larger levels of thrust.

The typical repeatability of the force measurements can be seen in Figure 22. In this figure, the data for three different runs can be seen to be within the accuracy bounds established earlier. Also shown in Figure 22 is a run in which the tunnel speed was held constant at $100 \mathrm{ft} / \mathrm{sec}$, as opposed to the standard velocities shown in Table 2. The data reveals that the effect of tunnel speed is within the repeatability band. This point will be discussed further in Chapter IV.

The accuracy of the interference drag data is somewhat less than that for the interference lift. This can be attributed to the small loadings which were encountered. The interference pitching moment data had similarly poor error characteristics. The pitching moment coefficient dependency on lift (transfer of reference axis to the quarter chord) and drag (drag-pitch interaction from the model being mounted above the balance centerline) was largely responsible for the uncertainty of the data. 


\section{CHAPTER IV}

\section{DISCUSSION OF THE RESULTS}

The aerodynamic characteristics of the two-dimensional wing with and without the jet operating are discussed in this chapter, including the effects of varying the freestream velocity (and consequently, wing Reynolds number). An interpretation of the jet-on aerodynamic characteristics is presented followed by a physical explanation of the observed behavior. Included is a comparison with infinite flat plate data and a discussion of the effects of the different flow and geometry parameters. Finally, a comparison with available theoretical predictions is made.

\section{Jet-Off Aerodynamic Characteristics}

The determination of the aerodynamic characteristics of the plain twodimensional wing, which is the reference case against which jet-on behavior is compared, proved to be more difficult than anticipated. Early in the experiment, an examination of the extensive spanwise pressure distribution available at $\mathrm{x} / \mathrm{c}=$ 0.45 (See Figure 13) revealed that the uniformity and the values of the surface pressure coefficients were strongly dependent on the magnitude of the freestream velocity. The non-uniformity which was manifested at the lower velocities suggested that the boundary layer was not transitioning uniformly. Hence, a transition strip (described in Chapter II) was installed at $\mathrm{x} / \mathrm{c} \simeq 0.05$ in an attempt to overcome this low Reynolds number effect. (The Reynolds number based on wing chord was about 
300,000 for $V_{i}=50 \mathrm{ft} / \mathrm{sec}$.) Figure 23 presents the spanwise pressure distribution with and without the transition strip installed. A. marked improvement in the uniformity of the pressure distribution is evident. However, there was still a significant difference in $\mathrm{C}_{\mathrm{p}}$ between $\mathrm{V}_{\mathrm{i}}=50 \mathrm{ft} / \mathrm{sec}$ and $\mathrm{V}_{\mathrm{i}}=100$ or $150 \mathrm{ft} / \mathrm{sec}$ in the vicinity of the wing centerline even with the boundary layer trip. An examination of the chordwise pressure distribution at $\mathrm{y}=0$ as done in Figure 24 shows this discrepancy to be peculiar to $\mathrm{x} / \mathrm{c}=0.45$ with the boundary layer trip installed. The results of Figure 24 are typical of the chordwise distributions at several span stations near the centerline and were considered acceptable. The lower surface spanwise pressure distribution with the boundary layer trip installed at several angles of attack is shown in Figure 25. Note that the general trend of the distribution is much the same for the various angles. In light of this, the non-uniformity in the spanwise pressures remaining after installation of the transition strip was attributed to model surface irregularities and no further attempt was made to improve the distribution. Furthermore, the pressure coefficients measured at $\mathrm{V}_{\mathrm{i}}=100 \mathrm{ft} / \mathrm{sec}$ were taken to be the jet-off values for the calculation of the interference pressure coefficient regardless of the tunnel speed at which the jet-on measurements were made (the judiciousness of this decision will be further discussed in the next section).

Chordwise pressure distributions for the angles of attack at which jet-on measurements were made are shown in Figure 26. Note that the lower surface adverse pressure gradient is constant for much of the chord and is relieved (to essentially zero at $\alpha=9^{\circ}$ ) with increasing incidence. Also, the indications of a boundary layer separation (incipient wing stall) on the upper surface can be seen 
in the behavior of the trailing edge $\mathrm{C}_{p}$ at $\alpha=9^{\circ}$. At this angle of attack, the upper surface $\mathrm{C}_{\mathrm{p}}$ at the trailing is becoming more negative.

The basic aerodynamic section coefficients for this model are presented in Figure 27. These data also were collected at $\mathrm{V}_{i}=100 \mathrm{ft} / \mathrm{sec}$. Note in particular the indications of an incipient stall at $\alpha=9^{\circ}$ which agrees well with the pressure measurements. However, in the course of the experiment, a suspicion arose concerning the constancy of the jet-off aerodynamic coefficients with changes in freestream velocity. Subsequently, lift, drag, and pitching moment measurements were made for the range of freestream velocities encountered during the jet-on testing. The results for lift coefficient are shown in Figure 28. These results tend to eliminate Reynolds number effects as the predominant factor since one would expect only a gradual increase in $\mathrm{C}_{\mathrm{L}}$ with increasing $\mathrm{V}_{\mathrm{i}}$ in this case. In an attempt to verify this result, the tunnel balance system was recalibrated (and the balance bridge arrangement improved) as discussed in Appendix B. The same measurements were again made after the recalibration with essentially identical results. The situation was finally explained through the use of flow visualization. An examination of Figure 29, which shows the variation in drag coefficient with freestream velocity, reveals an unusual behavior of $\mathrm{C}_{\mathrm{D}}$ which is particularly noticeable at $\alpha=12^{\circ}$. This behavior indicates a boundary layer separation again, but the reverse of what would be expected for a Reynolds number effect. To further investigate, rows of tufts were taped spanwise on the wing upper surface. Visual observations of the tufts were made at $\alpha=12^{\circ}$ as $V_{i}$ was increased. The tuft pattern appeared to indicate that the boundary layer separation point along the span 
was moving forward a significant distance as the velocity was increased from 30 $\mathrm{ft} / \mathrm{sec}$ to $70 \mathrm{ft} / \mathrm{sec}$. As the speed was further increased to $150 \mathrm{ft} / \mathrm{sec}$ the separation point seemed fairly constant--or perhaps moving aft a small distance. Such behavior substantiated the lift coefficient characteristics displayed in Figure 28.

The question of the forward moving separation point was finally resolved by an oil flow study. Figure 30 shows the wing upper surface for two different freestream velocities at $\alpha=9^{\circ}$. A weak boundary layer separation at 65-70 percent chord is clearly defined in both cases. (The thin horizontal lines are imperfections in the plastic sheet which occurred during its installation and should be ignored. The wing has been rotated to $\alpha=90^{\circ}$, leading edge up, for the picture.) However, the flow situation is vastly different for $\mathrm{V}_{\mathrm{i}}=32.8 \mathrm{ft} / \mathrm{sec}$ as shown in Figure 31a. A laminar separation bubble with subsequent flow reattachment is clearly visible in the photograph.

An explanation of the flow behavior on the upper surface was now possible. Wijker ${ }^{31}$ has noted that for the NACA 0018 airfoil without surface roughness, transition is always preceded by a laminar separation. For the current model, when the incidence is large enough a laminar separation occurs forward of the transition strip. Wijker has also stated that this laminar separation region reattached for the NACA 0018 profile. Furthermore, the point of reattachment moves toward the laminar separation point fairly rapidly as the Reynolds number is increased. This, of course, accounts for the apparent forward movement of the separation point in the current tuft study. The use of tufts to indicate flow separation was found to be misleading at the lower tunnel speeds. In the laminar separation 
bubble, the tufts appeared to be properly aligned and unmoving thus indicating attached flow. Conversely, as the reattachment point moved forward the tufts indicated this to be a moving separation point. The flow over the surface aft of the reattachment point can best be described as a "weak separation" using the terminology of Wijker which moves forward concurrently with the reattachment point as the Reynolds number is increased. This behavior then accounts for the unusual characteristics of the lift and drag coefficients shown in Figure 28 and Figure 29, respectively.

Summarizing, the jet-off characteristics of the wing were considered reasonably well understood and sufficiently documented at this point. The jet-off pressure distributions were acceptable since changes in surface pressures with jet operation were so large as to mask any difference in $\Delta \mathrm{C}_{\mathrm{p}}$ that could be attributed to the jet-off variation and, furthermore, the behavior of the laminar separation bubble was not altered by jet operation as discussed in the next section. Also discussed there is the procedure used in the data reduction to account for the variation of the aerodynamic force and moment coefficients with freestream velocity. Concluding, since the wing characteristics with the jet-off were acceptable as a reference datum, no further effort to improve these aerodynamic characteristics was expended.

\section{Jet-on Aerodynamic Characteristics}

The behavior of the aerodynamic characteristics with variation in freestream velocity were also considered for jet-on operation. Of primary importance is whether the changes induced by the jet are particular to the airfoil shape being 
tested. For example, if the laminar separation bubble previously discussed were to disappear or have significantly altered characteristics due to the presence of the jet, then the jet-on results observed at the lower Reynolds numbers would be of questionable value. Fortunately, this was not the case.

It must be noted, however, that the majority of the difficulties described in the previous section occurred for $\mathrm{V}_{\mathrm{i}}<100 \mathrm{ft} / \mathrm{sec}$. On the other hand, most of the jet-on testing was done for $\mathrm{V}_{i} \geq 100 \mathrm{ft} / \mathrm{sec}$ (see Table 2). Nevertheless, the following discussion is included to verify that the jet-on aerodynamic characteristics for the complete range of freestream velocities $\left(32.8 \leq \mathrm{V}_{\mathrm{i}} \leq 150 \mathrm{ft} / \mathrm{sec}\right)$ used in this experiment did not exhibit peculiarities which were particular to this model. In this discussion, comparisons are made at $\lambda=8$ since this is the largest value of the effective velocity ratio for which a $V_{i}=100 \mathrm{ft} / \mathrm{sec}$ was possible. This choice thus enabled comparisons to be made between a tunnel speed which yielded well behaved aerodynamic characteristics $(100 \mathrm{ft} / \mathrm{sec})$ and the lower tunnel speeds and their less favorable characteristics (less than $50 \mathrm{ft} / \mathrm{sec}$ ).

An examination of Figure 31 clearly shows the laminar separation bubble to be much the same at $\lambda=0$ and at $\lambda=8$. The flow pattern on the remainder of the wing is also very similar although this is somewhat obscured by the fact that a different oil mixture batch (more freely flowing) was used in Figure $31 b$.

An investigation of the effect of different freestream velocities on the interference surface pressures was also made. Figure 32 presents jet-on and jet-off chordwise pressure distribution at different tunnel speeds for $y=5.50$ inches. It can be seen that the interference increment is significantly larger than that due to 
a variation in freestream velocity. Recall from Figure 23 that the largest spanwise discrepancy in $\mathrm{C}_{\mathrm{p}}$ occurred near the wing centerline. To investigate the possible carryover to the jet-on case, a comparison of chordwise pressure distributions in the area of the wing centerline is shown in Figure 33. Clearly, the agreement between the two tunnel speeds is good. (except possibly in the immediate vicinity of the jet where the pressure gradients are quite large) and the magnitude of pressures induced by the jet mask any differences in the two cases (See Figure 32 for corresponding jet-off pressures). This is further evidenced by Figure 20 in which a direct comparison of the interference pressure contours at $\lambda=8$ for different freestream velocities reveals discrepancies to be minimal. Finally a spanwise comparison is presented in Figure 34 . The agreement is again acceptable in the region near the jet. Figure 34 also indicates that the region of surface pressure tap concentration (from $\mathrm{y}=0.0$ to $\mathrm{y}=16.0$ inches, see Figure 13 ) was adequate.

In light of these results, the decision to consider the jet-off pressure coefficients invariant with freestream velocity was appropriate. As stated earlier, this is particularly true since the vast majority of all jet-on pressure measurements were made at a $V_{i} \geq 100 \mathrm{ft} /$ sec (see Table 2) where the jet-off pressure distributions were well behaved. Furthermore, the experimental evidence indicates that those cases which were run at a $\mathrm{V}_{\mathrm{i}}<100 \mathrm{ft} / \mathrm{sec}$ can also be considered representative results.

Also note in Figure 34 that the available data indicates symmetric flow conditions with respect to the wing (and hence the jet) centerline. Each of the 
interchangeable panels containing the jet exit were instrumented to provide a check on the symmetry of the pressures. Furthermore, the flow visualization using oil (discussed later in this chapter) confirmed that symmetric conditions existed with the jet in operation.

The Reynolds number effect on the calculation of the interference force and moment coefficients was taken into account more directly. This was accomplished by considering the dependency of the jet-off aerodynamic coefficients on the freestream velocity (which is directly proportional to the Reynolds number). That is, in the data reduction, the jet-off values for $\mathrm{C}_{\mathrm{L}}, \mathrm{C}_{\mathrm{D}}$, and $\mathrm{C}_{\mathrm{M}}$ were used that corresponded to the freestream velocity at which the jet-on data was taken. This can be seen to be an acceptable procedure by examining Figure 22. In that figure a number of interference lift coefficient measurements were made for the same $\lambda^{\prime} \mathrm{s}$ but at different freestream velocities and the results are seen to be within the general repeatability of the force measurements.

The major hindrance to the accuracy of the interference force and moment data was the tunnel balance system. The main difficulty was in the balance zero returns and this is discussed in detail in Chapter $\Pi$ and Appendix B. These effects were somewhat more acute for the drag component and hence the pitching moment coefficient which had to be corrected with the drag reading since the model was mounted above the balance centerline. Because of this difficulty, the interference drag and pitching moment data should only be used for indicating general trends.

In the experiment, the geometric configurations investigated were:

$$
\mathrm{d}_{\mathrm{j}} / \mathrm{c}=0.10 \quad, \quad \mathrm{x}_{\mathrm{j}} / \mathrm{c}=0.25
$$




$$
\begin{array}{ll}
d_{j} / c=0.10 & , \\
d_{j} / c=0.10 & x_{j} / c=0.45 \\
d_{j} / c=0.20 & x_{j} / c=0.65
\end{array}
$$

(A nozzle assembly with a 0.75 inch jet, $\mathrm{d}_{\mathrm{j}} / \mathrm{c}=0.05$ was also fabricated but never tested because of the very low resulting thrust levels.) For each geometry, pressure measurements were made at $\alpha=0^{\circ}, 6^{\circ}$, and $9^{\circ}$ for $\lambda=2,4$, and 8 and for $\alpha=0^{\circ}$ at $\lambda=12$. The resulting interference surface pressure contours for each of these conditions is presented in Appendix C.

Measurements of the interference forces and pitching moments were also made at each of the above conditions. In addition, for each geometry and angle of attack, force and moment measurements were made for $\lambda=3,6,10$, and 12 and on occasion for $\lambda=2.5$. A summary of the interference lift, drag, and pitching moment data is presented in Appendix D.

\section{Comparison with Flat Plate Results}

A comparison of the interference surface pressure contours on the wing lower surface with $d_{j} / c=0.10, x_{j} / c=0.45$, and $\alpha=0^{\circ}$ and those on an infinite flat plate is made in Figure 35. The flat plate interference pressures shown are those measured by Mosher. ${ }^{15}$ Figure $35 \mathrm{a}$ is the comparison at $\lambda=12$ and, except in the immediate vicinity of the jet, the contours are very nearly identical. The comparison at $\lambda=8$ is made in Figure $35 \mathrm{~b}$ and, although the contours are not in as close agreement as in the $\lambda=12$ case, a distinct similarity is apparent. Of particular interest is the area just forward of the jet. Note the appearance as $\lambda$ 
is decreased of a growing region of positive interference pressure on the wing that does not exist on the flat plate. Finally, in Figure 35c, the comparison for $\lambda=4$ shows a gross disimilarity in the two cases. This difference is primarily concentrated in the region forward of the jet in which a significant spreading of positive interference pressure on the wing occurs that is rather localized on the flat plate to the immediate area in front of the jet. Also, the region of negative interference pressures aft of and lateral to the jet is somewhat more contained in the wing case.

The characteristics of the interference surface pressures on the wing are much the same for the other jet exit locations (See Appendix C). In all cases, the effect of increasing angle of attack is primarily to decrease the extent of the region of positive interference pressure forward of the jet. For the larger jet $\left(d_{j} / c=0.20\right)$, this positive interference pressure region becomes much more extensive for all angles of attack. That is, the spreading is more than proportionate to the increase in jet diameter. For $d_{j} / c=0.20$ and $\lambda>6$, the similarity with the flat plate data is approximately the same as for the $d_{j} / c=0.10$ case.

The existence and extent of a positive interference pressure region forward of the jet has also been reported by Williams and Wood. ${ }^{23}$ They commented on the results of a simple rectangular wing with a centrally located circular jet that at $\lambda=4$ exhibited a region of strong positive interference pressure ahead of the jet that had no counterpart in the corresponding flat plate pressure distribution.

This region is essentially responsible for many of the gross aerodynamic characteristics of the wing to be noted later. A discussion of the cause of the positive pressure region is given in the section on physical interpretation. 
This comparison of the interference surface pressures of the wing and the flat plate indicates that for $\lambda>8$ the jet issuing from the flat plate is a reasonable simulation of the lifting case. That is, the interference surface pressure contours on the wing lower surface are approximately the same as those on the infinite flat plate for identical values of the effective velocity ratio. At values of $\lambda<8$, the flow situation is vastly different. (Subsequent discussion will indicate that the flow undergoes this character change for $4>\lambda>8$ and eventually $\lambda \simeq 6$ is used as the approximate boundary.) This assertion can be further supported by comparing the oil flows at $\lambda=4\left(d_{j} / c=0.20\right)$ on the wing given in Figure 36 with those of the flat plate $^{15}$ shown in Figure 37 . The dissimilarity of the wake may be very significant in this comparison. Further evidence that the character of the interference flow field changes for $\lambda<6$ is given in the next section.

\section{Parametric Comparison of the Aerodynamic Interference Characteristics}

This comparison is made using the interference lift coefficient which is expressed as a function of the effective velocity ratio for the various geometric parameters for which data was accumulated. In this presentation, as in Appendix D, the parameter $1 / \lambda$ is used as the independent variable. This parameter which ranges from zero in hovering flight to a practical maximum of about 0.5 is considered more natural in describing the interference aerodynamics of transitional flight. In each case, the reason for the observed behavior of the aerodynamic interference coefficients is explained in terms of the corresponding interference pressure contours on the wing lower surface (see Appendix C). The effect of jet operation on the wing upper surface was quite small for the $d_{j} / c=0.10$ case. Characteristically, levels 
of surface pressure coefficients in the range $-0.10<\mathrm{C}_{\mathrm{p}}<0$ were induced by the jet on the upper surface and these levels did not vary appreciably with changes in effective velocity ratio, jet exit location, or angle of attack. Consequently, the behavior of the aerodynamic interference coefficients can be explained by the observed changes in the interference pressure distribution on wing lower surface. However, somewhat more pronounced suction pressures are induced on the upper surface for $d_{j} / c=0.20$. Hence, the interference pressure contours on the wing upper surface are included in Appendix C for this case.

No attempt was made at integrating the wing surface pressure distributions to obtain interference force coefficients because it was felt that the arrangement of the pressure tap locations was not suitable for such a procedure. A physical interpretation of the characteristics discussed here is reserved for the next section.

The variation of interference lift coefficient with the effective velocity ratio is presented in Figure 38 for the three angles of attack. In this figure, a significant lift augmentation occurs for $\lambda<6$ (i.e., $1 / \lambda>0.17$ ) and the augmentation effect is decreased for increasing angle of attack. The reader is reminded that zero interference lift corresponds to $\frac{\Delta \mathrm{L}}{\mathrm{T}}=1.0$ (i.e. for a lift loss, $\frac{\Delta \mathrm{L}}{\mathrm{T}}<1.0$ and for a lift augmentation, $\frac{\Delta \mathrm{L}}{\mathrm{T}}>1.0$ ). Note that for all angles of attack a reversal of the observed trends occurs for $\lambda \simeq 6$. A study of the corresponding interference surface pressure distribution indicates that the region of positive interference pressure forward of the jet is mainly responsible for the lift augmentation. (Reiterating, only minor changes characterized by small induced negative interference pressures that did not vary with $\lambda$ were observed on the upper surface.) The extent of the positive 
interference pressure region decreases correspondingly with increasing angle of attack. Note also that the adverse interference effect for $\lambda \geq 6$ is alleviated somewhat by increasing incidence. For this alleviation, which can be considered a second order effect, a very slight increase in the extent of the positive pressure region and a correspondingly small decrease in the region of negative interference pressures aft of and lateral to the jet is noticeable.

Figure 39 shows typical trends for the interference drag coefficient and the interference pitching moment coefficient. The significant drag rise and increase in adverse pitching moment is characteristic of values of $\lambda$ in which the lift augmentation was observed. These trends are consistent with the aforementioned behavior of the interference surface pressures. Again, the reader is cautioned not to interpret the interference drag and pitching moment data quantitatively because of the possibility of significant errors (see Chapter III).

The effect of jet exit location on the interference lift coefficient is presented in Figure 40. Not surprisingly, the lift augmentation increases as the jet exit location is moved aft. This can be directly attributed to the increased area available for the positive interference pressures forward of the jet. (However, on the basis of flat plate data one would have attributed this effect to a decrease in the area available to the negative interference pressures aft of the jet instead of the situation described above.) Again, for the cases of alleviated detrimental effects for $\lambda \leq 6$ (this occurs for $\mathrm{x}_{\mathrm{j}} / \mathrm{c}=0.25$ and $\mathrm{x}_{\mathrm{j}} / \mathrm{c}=0.65$ ) a slight increase in the extent of the positive pressure region and a minor contraction of the negative pressure region is ascertainable. 
Carter $^{28}$ has observed similar behavior of the interference lift coefficient with jet exit location. He reported interference lift losses that were reversed at $\lambda \simeq 6$ and also an increasing lift augmentation as the jet exit location was moved aft. Significantly, the lift augmentation noted in that work was not nearly of the magnitude reported here. This is probably a result of Carter's experimental configuration in which the nearest the jet exit plane could be positioned was 0.64 wing chords beneath the wing chordal plane. In that work, chordwise pressure distributions at the span station which contained the jet exit centerline showed only small positive interference pressures forward of the jet exit at $\lambda=4$. The magnitude of those interference pressure coefficients for $\mathrm{x}_{\mathrm{j}} / \mathrm{c}=0.75$ was about half that reported in the present investigation at $\mathrm{x}_{\mathrm{j}} / \mathrm{c}=0.65$. Conversely, Carter reported much larger positive interference pressure coefficients forward of the jet for $\mathrm{x}_{\mathbf{j}} / \mathrm{c}$ $=0.75$ at $\lambda=10$. These comments indicate that the interference flow characteristics are certainly different when the jet exit plane does not coincide with the wing surface.

Finally, Figure 41 presents the effect of jet exit diameter on interference lift coefficient. The larger jet $\left(\mathrm{d}_{\mathrm{j}} / \mathrm{c}=0.20\right)$ provides a comparatively favorable effect throughout the range of $\lambda$. This is again attributed to the region of positive interference pressure forward of the jet for $\lambda<6$. In the case of $\mathrm{d}_{\mathrm{j}} / \mathrm{c}=0.20$, this region is much more extensive than a direct scaling of the exit diameters would predict. However, an increase in the suction pressures induced by the jet on the wing upper surface is primarily responsible for the favorable effect in the $\lambda>6$ range. 
An examination of Figures 38,40 , and 41 shows that in all cases a reversal of the trends discussed occurs at $\lambda \simeq 6$. This fact strengthens the allegation made earlier that the character of the interference flow undergoes a change at about this velocity ratio. For $\lambda>6$, the situation is reminiscent of the infinite flat and for $\lambda<6$ the character is decidedly different.

\section{Physical Interpretation}

The experimental agreement of the wing aerodynamic characteristics with the flat plate case for $\lambda>6$ is quite distinct. This includes the interference surface pressure contours and the corresponding detrimental effect on the interference lift which would be predicted by an integration of the surface pressures on the flat plate. For these higher effective velocity ratios, the dominance of the jet compared to any effect of wing/crossflow interaction is evident. In these cases, the jet induces small suction pressures on the upper surface of the wing that were not sensitive to changes in wing angle of attack or jet exit location. These suction pressures are a result of the downwash field that is created by jet operation.

In this range of higher effective velocity ratios, the observed small increase in the region of positive interference pressure forward of the jet and the moderate contraction of the negative interference pressure region lateral to the jet with increasing angle of attack can be explained by appeal to an "apparent" $\lambda$ change. This is done by recalling that the jet exit dynamic pressure was set by assuming the jet exit static pressure to be the freestream static value. The effect of this approximation can be more easily seen by considering the isentropic relationship, 


$$
M_{j}^{2}=\frac{2}{\gamma-1}\left[\left(\frac{p_{o p}}{p_{s}}\right)^{\frac{\gamma-1}{\gamma}}-1\right],
$$

where $\mathrm{p}_{\mathrm{op}}$ is the plenum chamber stagnation pressure and $\mathrm{p}_{\mathrm{S}}$ is the local static pressure at the jet exit plane. The technique used in setting the stagnation pressure always resulted (regardless of the angle of attack or jet exit location) in the same value for $\mathrm{p}_{\mathrm{op}}$ since this was referenced to the freestream static value. However, as the angle of attack is increased the actual local static pressure at jet exit is also increased (see Figure 26) and hence a lower jet exit Mach number and correspondingly a lower jet exit dynamic pressure results. Thus, the effective velocity ratio also decreases. This small decrease in the "apparent" velocity ratio then accounts for the observed pressure changes, as can be seen in the surface pressure trends noted for changes in $\lambda$ in Reference 15. This effect is quite small and should certainly be considered second order.

For $\lambda>6$, similarly small increases in the region of positive interference pressure forward of the jet and the contraction of the negative interference pressure lateral to the jet were observed for $\mathrm{x}_{\mathrm{j}} / \mathrm{c}=0.25$ and 0.65 (when compared with $\left.\mathrm{x}_{\mathrm{j}} / \mathrm{c}=0.45\right)$. Figure 26 reveals an increase in the local static pressure with respect to the $x_{j} / c=0.45$ values for these two jet exit locations. Therefore, the "apparent" velocity ratio effect can again be identified as the underlying cause.

For the lower effective velocity ratio range $(\lambda<6)$, the character of the interference flow is decidedly different. The hypothesis advanced here is that the lifting jet-crossflow interaction begins to exhibit the characteristics of a jet-flap. 
A jet flap provides a lift augmentation by inducing an increased effective angle of attack through downwash while simultaneously allowing a non-zero pressure differential at the wing trailing edge. ${ }^{32}$ This latter effect is possible because the deflected jet-flap can support a pressure differential (the so-called supercirculation effect). A direct result is an alleviation of the adverse pressure gradient on the wing upper surface thus delaying any boundary layer separation.

The experimental evidence that this "jet-flap effect" is occurring is emphasized by Figures $42,43,44$, and 45 . In Figure $42 \mathrm{~b}$, note that the upper surface streamlines (in the vicinity of the wing centerline) are showing a definite influence of the jet operation. The effect at $\alpha=9^{\circ}$ is more dramatic as shown in Figure 43 . For the upper surface (Figure 43b), the local prevention of boundary layer separation is obvious. More surprising, however, is the standing vortex aft of the jet exit shown in Figure 43a. The only difference in the conditions between Figure 42 and Figure 43 is the increase in angle of attack which must then account for the vortex formation. (The presence of this standing vortex was not discovered until the very end of the experimental program because it was felt that no oil flow studies should be made until all the surface pressure data had been gathered since the possibility of having the oil mixture migrate into the surface pressure lines existed. The unavailability of additional wind tunnel time prohibited further investigation.) Finally, evidence that a non-zero pressure differential at the trailing edge exists locally in the region aft of the jet is presented in Figure 44 .

Collectively, these facts indicate that the behavior of this lifting jet is similar to a jet-flap installation. However, it must be noted that the fundamental 
mechanisms responsible for this behavior may be quite different in the two cases. That is, the principal mechanism of the jet-flap is the solid blockage effect that the jet momentum efflux provides. In the case of the lifting jet, the entrainment effect may be more significant. Nevertheless, the following discussion is based on the analogy between the lifting jet and the jet-flap.

The behavior of the previously noted aerodynamic characteristics can now be more fully explained. The region of positive interference pressures forward of the jet is thought to be a direct result of the induced downwash which is characteristic of jet-flap installations. The magnitude of these pressure changes can be seen to be much in line with those reported by Dimmock ${ }^{33}$ in which an elliptical profile with a pure jet-flap was investigated. A comparison of his results with a chord-wise pressure distribution of the present investigation for similar effective velocity ratios is presented in Figure 45 (the parameter of importance in the jetflap case is the jet momentum coefficient which in the cited example corresponded to a $\lambda \simeq 2$ ). Note that the positive interference pressures forward of the jet are nearly identical in both cases up to $x / c \simeq 0.35$. The interference pressures become more positive for the present study as the jet exit location $\left(x_{j} / c=0.65\right)$ is approached but this deviation should be expected since Dimmock's jet-flap was located at the trailing edge. This comparison demonstrates the increase in the extent of the induced positive pressures may be attributable to the jet induced downwash. By noting that moderate positive interference pressures forward of the jet already exist for the flat plate case at these effective velocity ratios, it can readily be deduced that a moderate induced angle of attack would produce the positive interference pressures 
observed on the wing.

The attenuation of the positive interference pressure region with increasing angle of attack can also be explained in light of the jet-flap concept. Alexander and Williams ${ }^{34}$ have reported that the incremental induced downwash angle due to jet-flap operation exhibits a slight decrease with increases in wing incidence. This is, of course, for the case of a constant jet angle with respect to the chordal plane. Hence, for the present investigation, this decrease in the magnitude of the "jetflap effect" with increasing angle of attack is not unexpected.

Another characteristic of the jet-flap is the induction of large suction pressures (particularly near the leading edge) on the wing upper surface. Only small induced suction pressures were noted in the present investigation for $d_{j} / c=0.10$. The magnitude of the difference in the upper surface interference pressure coefficients for the two cases can be clearly seen in Figure 45. The absence of large suction pressures was attributed to the rather poor jet-flap installation the current configuration affords. That is, the lifting jet efflux is much too far forward to be a truly effective jet-flap. However, for the larger jet $\left(\mathrm{d}_{\mathrm{j}} / \mathrm{c}=0.20\right)$, the interference pressure coefficients on the upper surface displayed the suction effect to a greater degree. The magnitude of these pressures was again much less than an efficient jet-flap would produce (see Appendix C). This effect was also evidenced for $\lambda>6$ and accounted for the improvement of the detrimental interference lift between $d_{j} / c=0.10$ and $\mathrm{d}_{\mathrm{j}} / \mathrm{c}=0.20$. The "apparent" velocity ratio effect previously discussed for $\lambda>6$ was also a contributing factor in the case of the larger jet since increases in the angle of attack tended to attenuate the detrimental interference lift. These 
experimental results indicate that the "jet-flap effect" is more pronounced for the larger jet exit diameter.

It also must be remarked in light of the insensitivity to changes in flow conditions of the small induced suction pressures on the upper surface that the positive interference pressure region is the dominant characteristic of the flow which is responsible for the lift augmentation. Further evidence of this is contained in Figures 42 and 43. In Figure 43b, at $\alpha=9^{\circ}$ the local improvement in the flow over the wing upper surface is considerable when compared with Figure $42 \mathrm{~b}$. Yet the lift augmentation is less in the $\alpha=9^{\circ}$ case indicating that the decrease in the positive interference pressure region overshadows the upper surface effect.

Finally, it should be noted that the conclusion that the lift augmentation provided by the positive interference pressure region forward of the jet may be induced by a downwash field is contrary to the opinions of Williams and Wood. ${ }^{23}$ Commenting on their preliminary work with a jet issuing from a plain rectangular wing at a $\lambda=4$, they expressed the opinion that no surface vorticity distribution could be expected to account for the observed increases in the positive interference pressure region on the wing forward of the jet which had no counterpart in the corresponding flat plate pressure distribution.

\section{Comparison with Theoretical Results}

Wooler, and Wasson and Kao provided theoretical results for the $\mathrm{x}_{\mathrm{j}} / \mathrm{c}=0.45$

*The assistance of P. G. Wooler and of Wasson and Kao, all of the Northrop Corporation, Aircraft Division, in providing theoretical calculations for one of the experimental test cases is gratefully acknowledged. Methods used for these calculations were developed for the Air Force Flight Dynamics Laboratory, WrightPatterson Air Force Base, Ohio, under contract F-33615-69-C-1602. 
and $d_{j} / c=0.10$ configuration. Calculations were made at $\alpha=0^{\circ}$ for $\lambda=2,4$, and 8. The results of Wooler utilize the method of Reference 29 .

A comparison with experiment is complicated somewhat by the fact that the method of Reference 29 presents results as interference pressure loadings. This quantity differs from the interference surface pressure coefficient primarily because any tangential velocity induced by the jet on the wing surface is not included. Furthermore, the loading distribution is a result of the incremental difference on the upper and lower surface pressures. Wooler ${ }^{29}$ indicates that the induced tangential velocity is identical on the upper and lower surface and hence the tangential velocity effect is cancelled in the interference loading distribution. Since the present experimental results indicate only small changes in the interference pressure distribution on the wing upper surface for $d_{j} / c=0.10$, it was considered appropriate to compare the experimental interference pressure distribution on the lower surface directly with the theoretical interference pressure loadings. The chordwise interference pressure loadings supplied by Wooler were plotted by machine to obtain contours using the technique described in Chapter III.

The comparison is made for $\lambda=8$ in Figure $46 \mathrm{a}$, for $\lambda=4$ in Figure $46 \mathrm{~b}$, and for $\lambda=2$ in Figure $46 \mathrm{c}$. At $\lambda=8$ the theoretical calculation fails to adequately predict the positive interference pressure contours forward of the jet. The agreement in the immediate vicinity of the jet is poor and Wooler ${ }^{29}$ states that the theoretical model is such that a good representation of the flow in this region should not be expected. The comparison at $\lambda=4$ exhibits characteristics that are much the same. The theoretical results for $\lambda=2$ predict the existence of the region of 
positive interference pressure forward of the jet. This fact indicates that the downwash field produced by the jet which induces these positive interference pressures may be amenable to a theoretical treatment. Wooler's theory is essentially an application of conventional lifting surface theory once the downwash field induced by the jet is predicted by replacing the jet with a sink-doublet distribution.

Finally, a comparison with the theoretical calculations of Wasson and Kao 35 is presented in Figure 47. In this case, the interference chordwise pressure disributions supplied were used to determine the contours which were again machine plotted. The essence of this theoretical model is the calculation of the complex velocity potential function required to negate the jet induced velocity field over sections of the wing. The agreement with the experimental results can be seen to be unsatisfactory, particularly in regions forward of the jet.

It should be noted that both of these theoretical models are based on a finite span wing. Hence, these calculations were made using an assumed wing span of 50 inches.

A possible reason for the discrepancy in the comparison between Wooler's results and those of the experiment may be in the application of the conventional lifting surface theory which he used. Williams and Wood $^{29}$ have noted that the available theoretical methods for estimating wing loadings seem quite inadequate to cope with the large downwash variations induced by the jet vorticity.

In summary, the comparison of the interference surface pressure contours of the wing with the available theory indicates that considerable improvements in the theoretical models are needed. 


\section{CHAPTER V}

\section{CONCLUSIONS}

An analysis of the data collected in this experiment has led to the following major conclusions:

1) A comparison of the interference surface pressure distribution of the wing with that of the flat plate indicates that such flat plate results are not wholly applicable when the jet efflux is from a lifting surface. The discrepancy in these interference pressures is quite small for values of the effective velocity ratio larger than six. However, for effective velocity ratios less than six in the lifting case a large increase occurs in the extent of the region of positive interference pressure forward of the jet which has no counterpart on the infinite flat plate.

2) Interference lift losses were observed for all jet exit geometries when the effective velocity ratio was greater than six. A lift augmentation in which the favorable interference lift was significantly larger than the installed thrust occurred for effective velocity ratios less than six and was attributed to the extensive increases in the region of positive interference surface pressure forward of the jet. This lift augmentation was accompanied by a large interference drag rise and an adverse (became more positive) increase in the interference pitching moment.

3) The effect of jet operation on the wing upper surface for $d_{j} / c=0.10$ was 
characterized by small induced suction pressures, the level of which were insensitive to changes in effective velocity ratio, angle of attack, or jet exit location. Consequently, changes in the lower surface interference pressure distribution were responsible for the aerodynamic interference behavior observed. Somewhat larger suction pressures were induced on the upper surface for $d_{j} / c=0.20$ which were sufficient to alleviate the lift losses normally observed for $\lambda>6$.

4) Increases in the wing angle of attack result in a decrease in the magnitude of the lift augmentation for $\lambda<6$ because of a corresponding shrinkage in the extent of the positive interference pressure region forward of the jet.

5) The lift augmentation is magnified as the jet exit location is moved aft thus providing an increase in the area available to the positive interference surface pressure region forward of the jet.

6) As the jet diameter/wing chord ratio is increased, the lift augmentation becomes moderately larger due to a wider spreading of the forward region of positive interference pressure for the larger jet.

7) Changes in wing angle of attack or jet exit location have only a moderate effect on the magnitude of the detrimental interference lift losses which occur for values of the effective velocity ratio in excess of six. The data indicates that the interference characteristics predicted from infinite flat plate results are adequate in the $\lambda>6$ range.

8) As the effective velocity ratio is decreased from six, the interaction of the lifting jet efflux and the wing begins to exhibit behavior similar to a jet-flap. The 
"jet-flap effect" is thought to be responsible for the behavior of the positive interference pressure region forward of the jet which is the dominating factor in the lift augmentation.

9) The comparison of the interference surface pressure contours of the wing with the available theory indicates that considerable improvements in the theoretical model are needed. 


\section{CHAPTER VI}

\section{RECOMMENDATIONS}

\section{Present Configuration}

Several improvements in the general test arrangement could be made that would provide an increase in the accuracy and repeatability of the data.

1) The described technique for bridging the wind tunnel balance with the air supply hoses was the apparent source of small shifts in the balance zero. This was particularly significant for the drag and pitching moment components for which the measured loadings were small. An improvement in the balance bridge would be afforded by axially aligning a grounded supply pipe with the existing supply pipe which passes through the pillow block. These pipes would be connected with a U-shaped trapeze--the vertical components being fabricated from wire reinforced vacuum hose and the horizontal link being plain steel pipe with elbows at each end. Such a design would also fully eliminate any momentum tares from the system. ${ }^{*}$ This, of course, would have to be done on both sides of the model and hence the bridge on the tunnel north side would have to be designed around the balance sideforce link.

2) Although the internal balance used to determine the jet thrust was generally

The author is grateful to Mr. C. V. Williams of the Lockheed-Georgia Company for this suggestion. 
accurate to within \pm 2.0 percent of the applied load, problems were occasionally encountered which indicated the floating ribs were binding on the wing spars. This could be eliminated by replacing the pins which permit the floating ribs to rotate with a more sophisticated bearing arrangement. Also, future calibrations of the internal balance should be made with a dental dam seal installed at the jet exit.

3) Finally, an improvement to the jet-off aerodynamic characteristics of the wing at low freestream velocities should be made. The elimination of the laminar separation bubble on the wing upper surface should be a prime objective. This could possibly be accomplished by changing the surface roughness--either by increasing the size of the glass spheroids used as roughness or by increasing the chordwise width of the transition strip or both. Of course, the major difficulties encountered were a result of the choice of a NACA 0021 airfoil profile for the wing, for the reasons which have been discussed. The profile shape would be virtually impossible to modify without essentially redesigning and rebuilding the entire model but were it possible the author recommends consideration be given to a more conventional (perhaps a six series) airfoil shape.

The serendipitous standing vortex at the trailing edge of the wing when $\lambda=2, \mathrm{~d}_{\mathrm{j}} / \mathrm{c}=0.20, \mathrm{x}_{\mathrm{j}} / \mathrm{c}=0.45$ and $\alpha=9^{\mathrm{O}}$ (see Figure 43a) was found at the end of the experiment and prior wind tunnel commitments made it impossible to investigate this phenomenon more thoroughly. Additional testing to determine the conditions at which this vortex occurs and the extent of its influence on the interference flow is 
certainly merited.

It would also be useful. to make some measurements of the flow field off the wing surface with a determination of the centerline of the jet plume and the spreading of the jet being especially interesting. The jet plume could be compared with that measured for the infinite flat plate case to determine any differences.

\section{Modifications for Additional Configurations}

The testing of jet exit geometries different from those used in this experiment would be a straightforward extension, with non-circular jets and multiple jets being of prime interest. In addition to fabricating a nozzle assembly compatible with the existing plenum chamber, each exit geometry would require a new wing panel including the installation of the 48 surface pressure taps in the vicinity of the jet exit.

Investigations of a jet exiting either from a wing pod or a simulated fuselage would also be fairly easy to accomplish. The pod or fuselage must be fabricated to fit the existing two-dimensional model and the major difficulty would be in extending the existing pressure leads from the wing surface to the new pod or fuselage surface.

Lastly, the design of the two-dimensional wing is such that the trailing edge section (aft 2.82 inches of the wing) is completely removable. Hence, a modification to provide a simple flap for future experiments could be easily accomplished. 


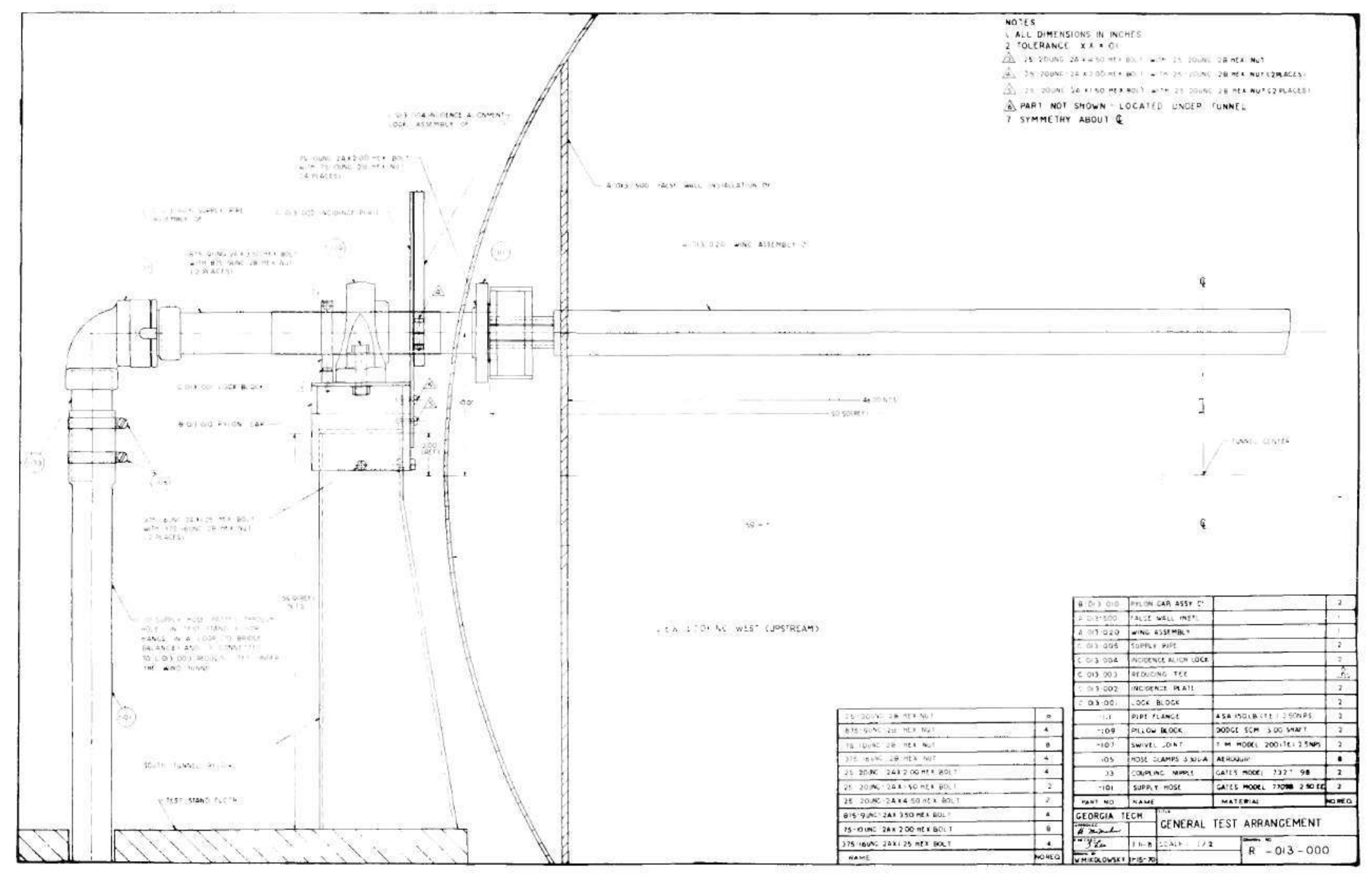

Figure 1. General Test Arrangement. 


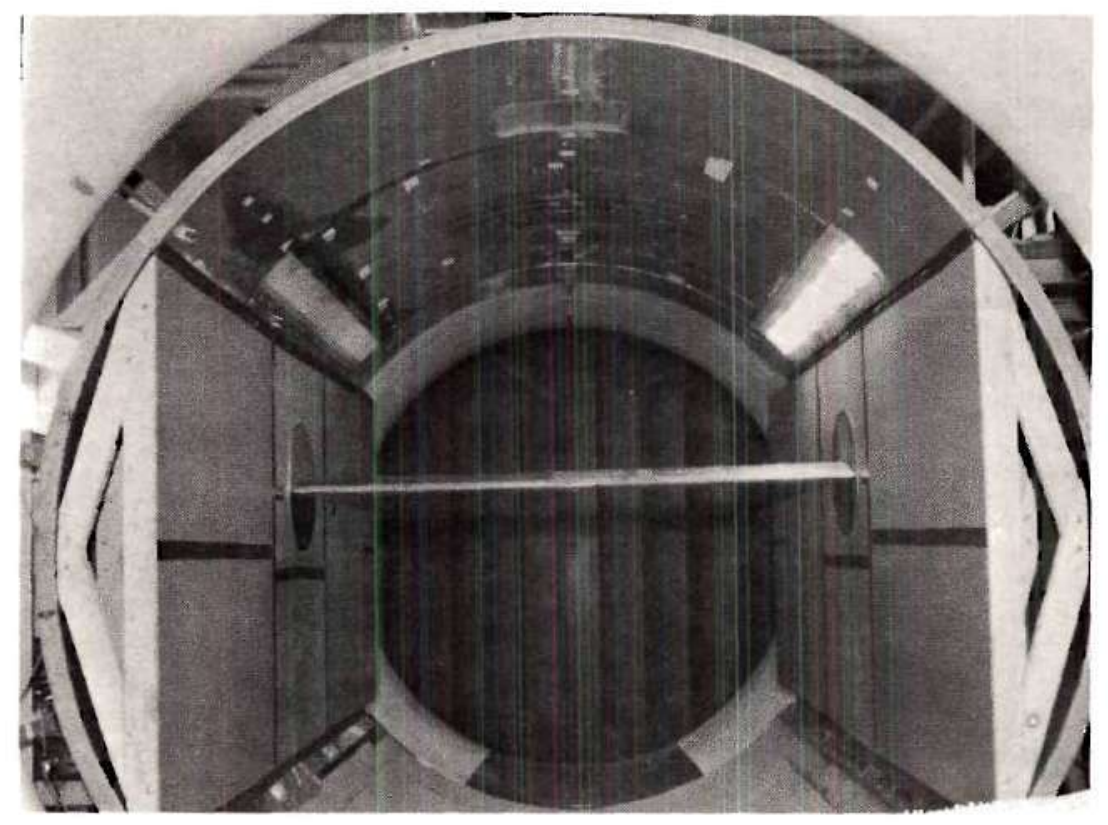

Figure 2. Model as Installed in Tunnel

(View Looking Upstream)

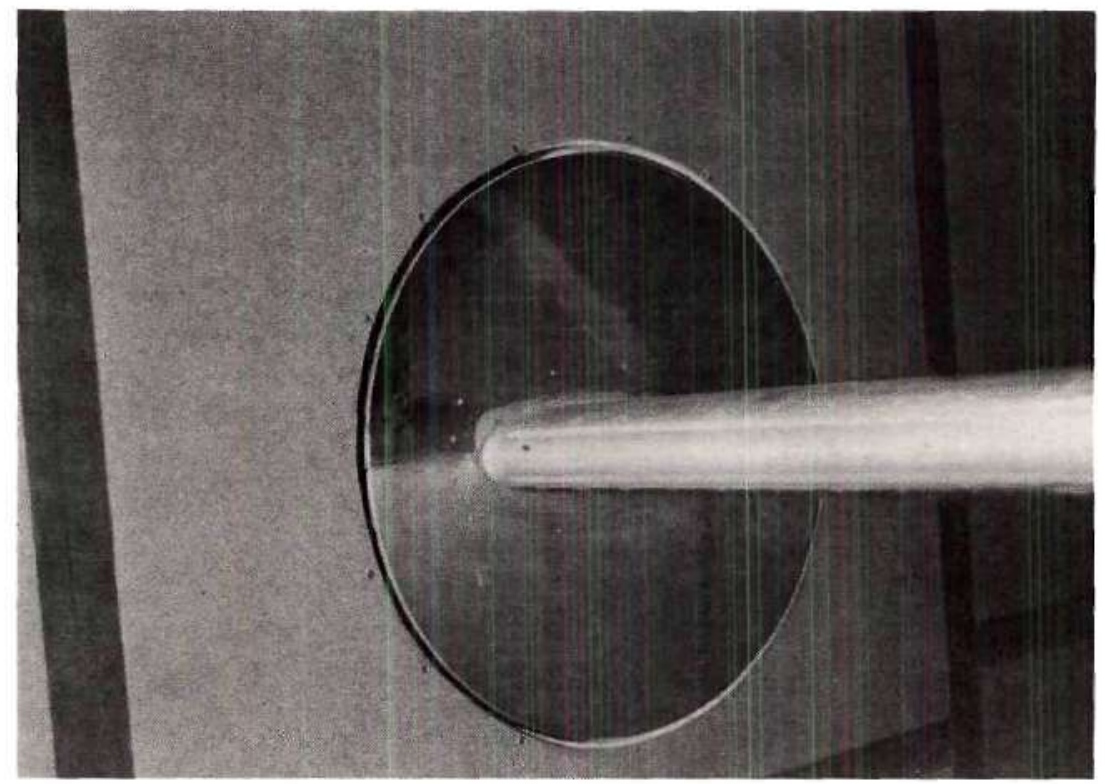

Figure 3. Wing Endplate. 


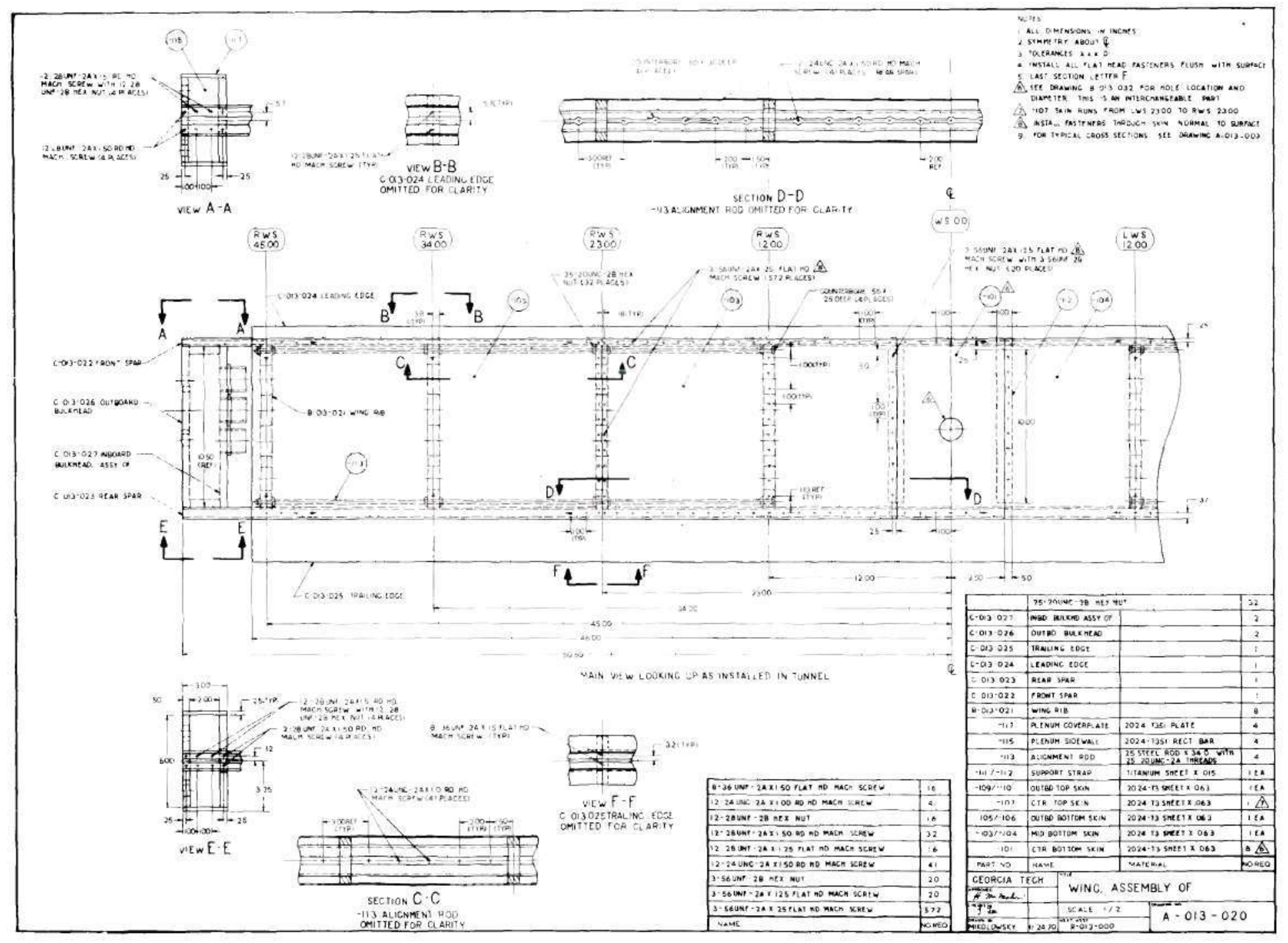

Figure 4. Wing Assembly. 


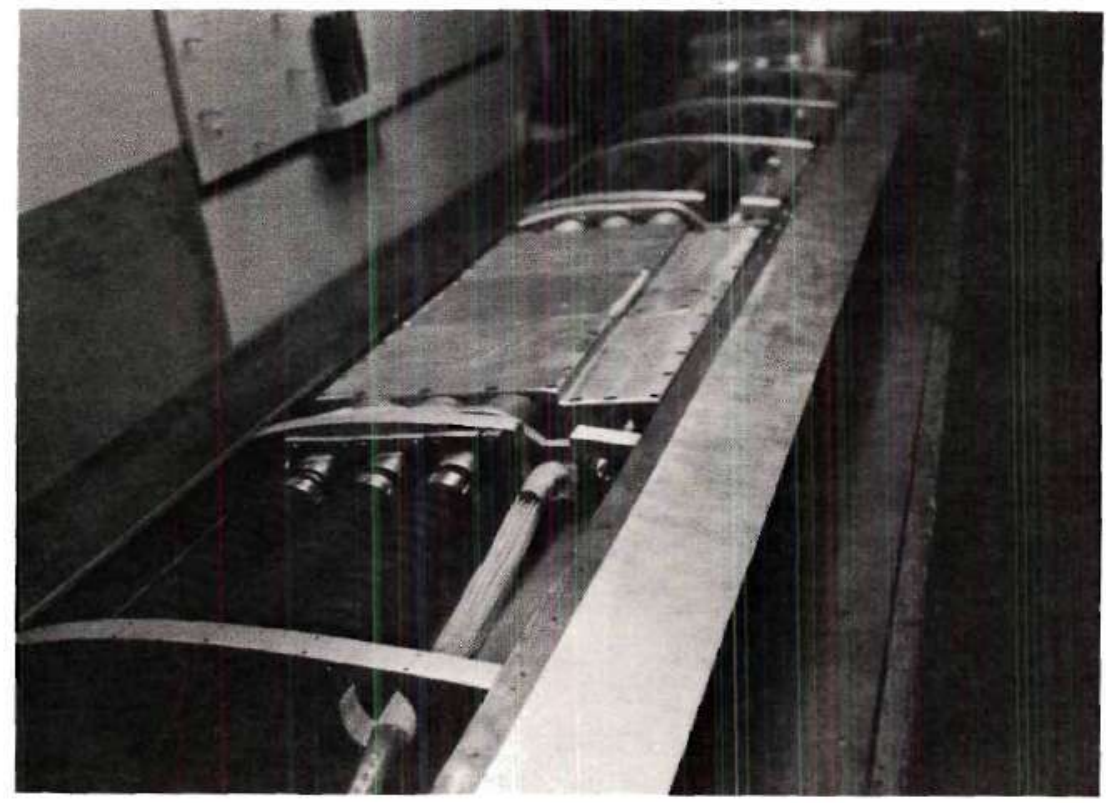

Figure 5. Wing with Skin Panels Removed.

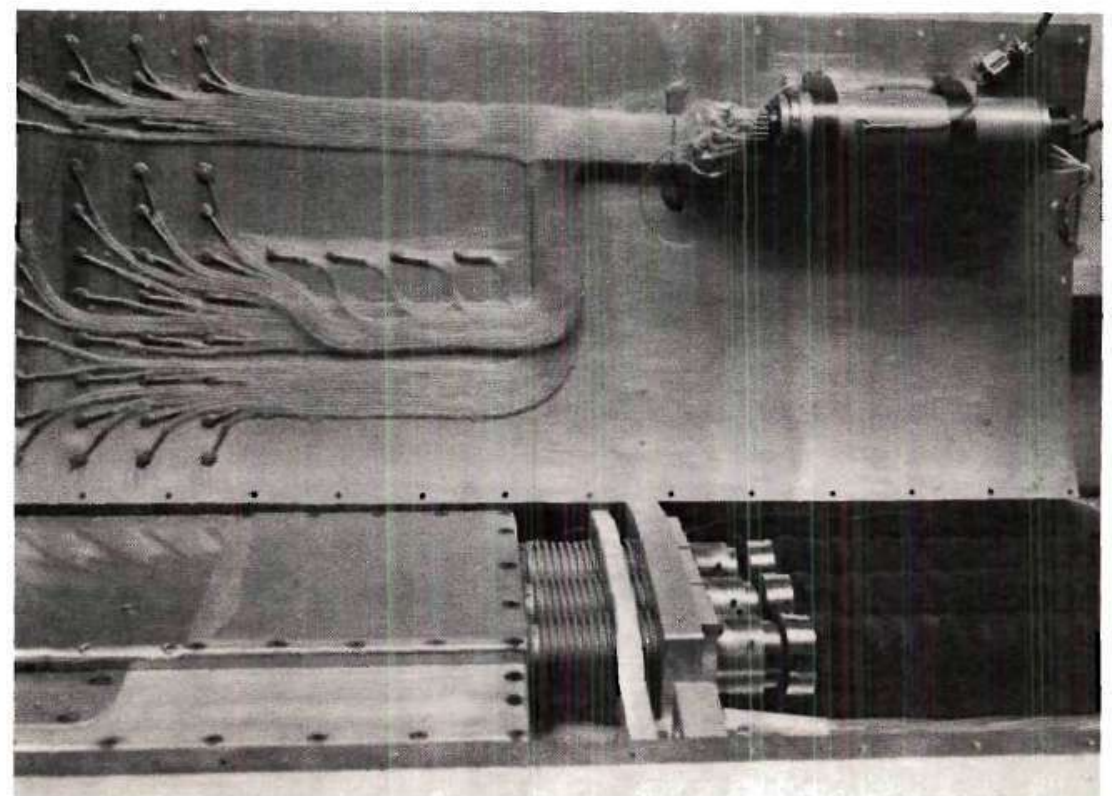

Figure 6. Typical Center Wing Skir Panel. 


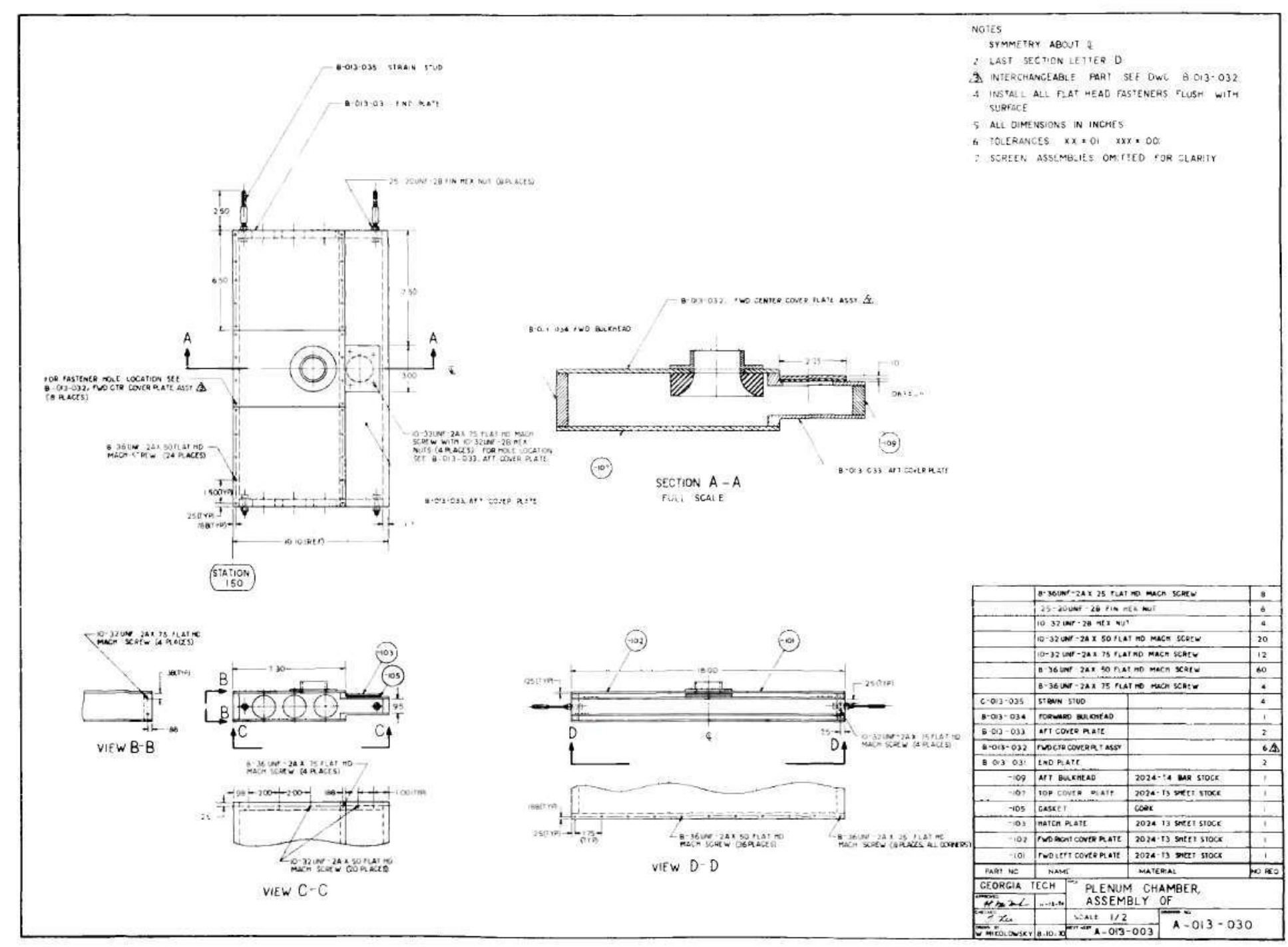

Figure 7. Plenum Chamber Assembly. 


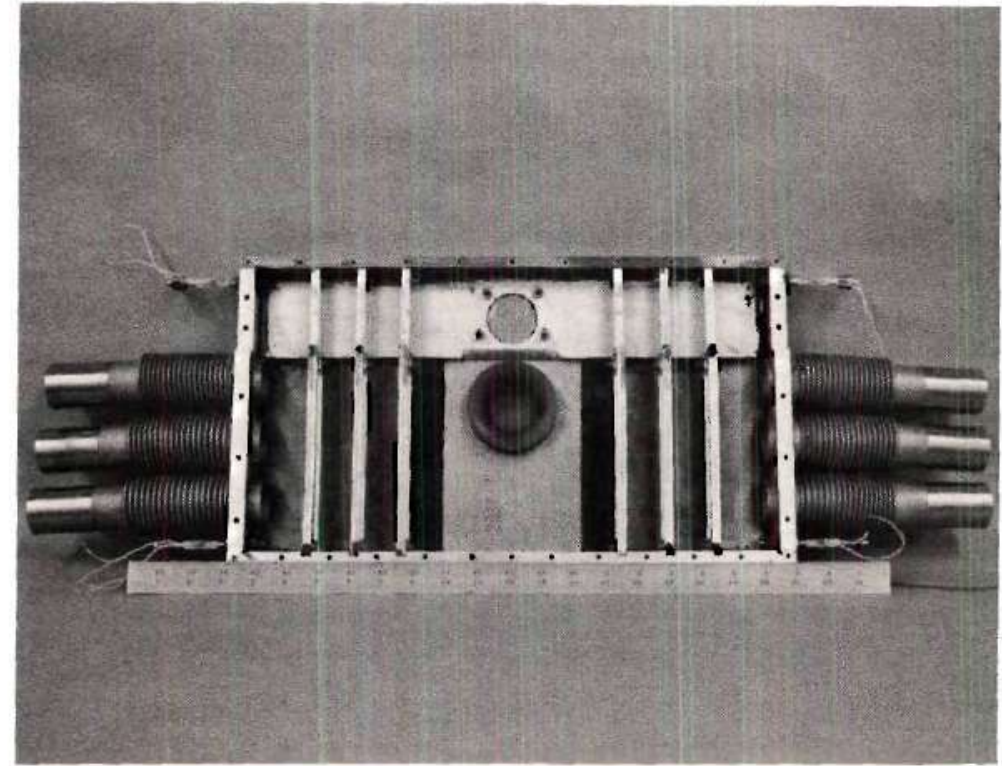

Figure 8. Plenum Chamber with Upper

Surface Removed.

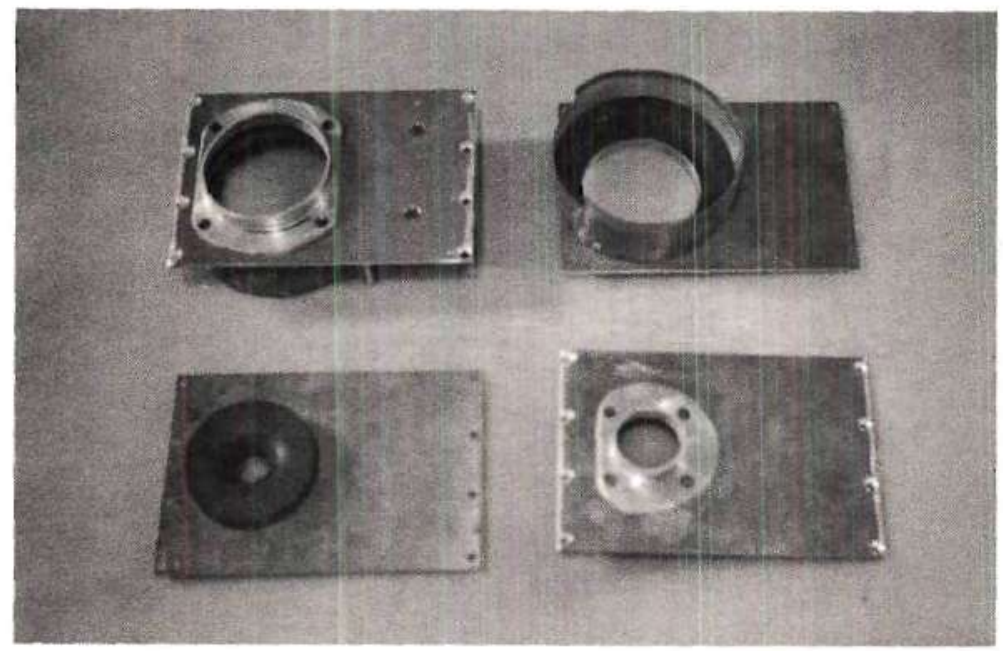

Figure 9. Typical Jet Nozzles. 


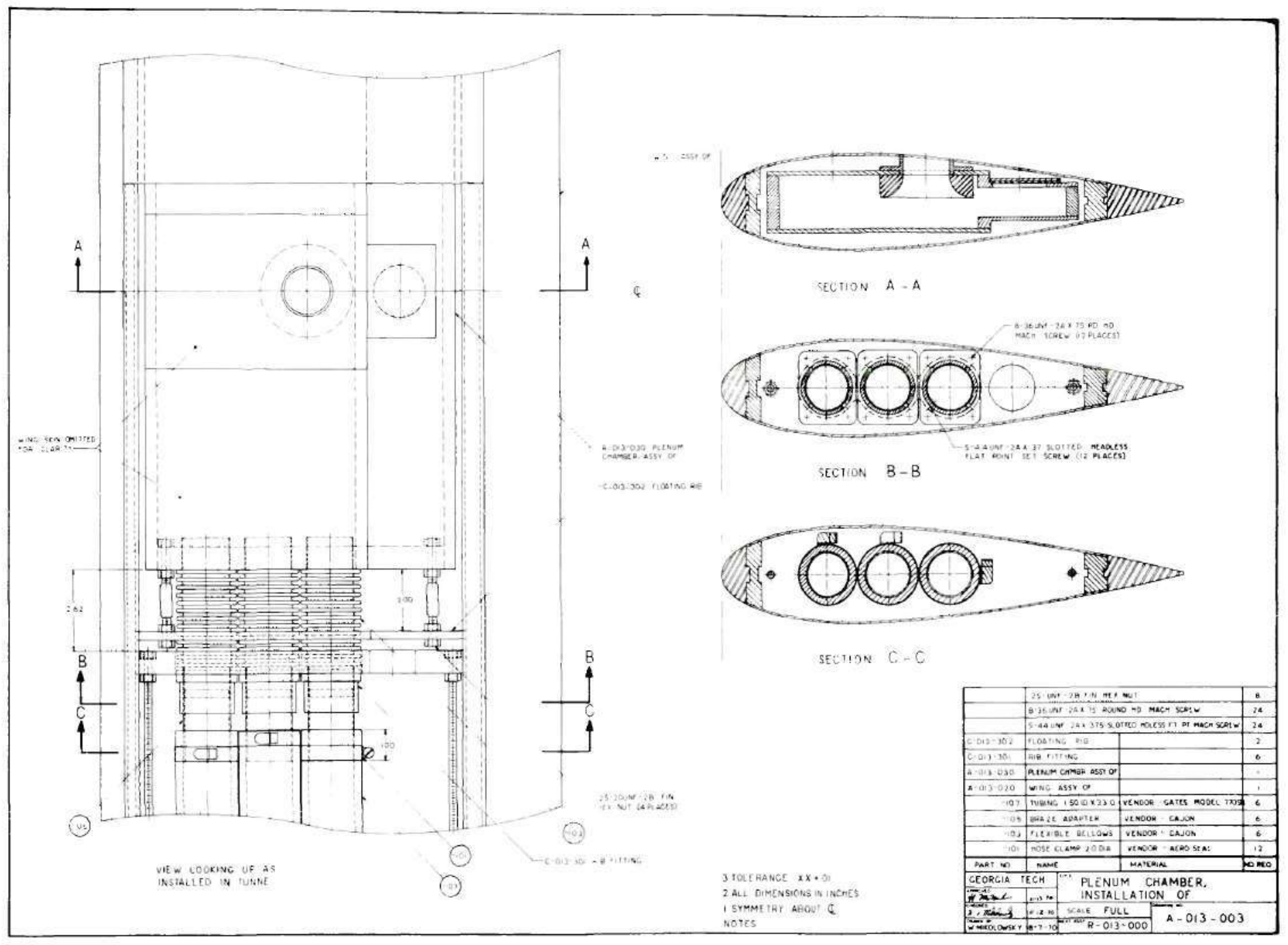

Figure 10. Installation of the Plenum Chamber in Wing. 


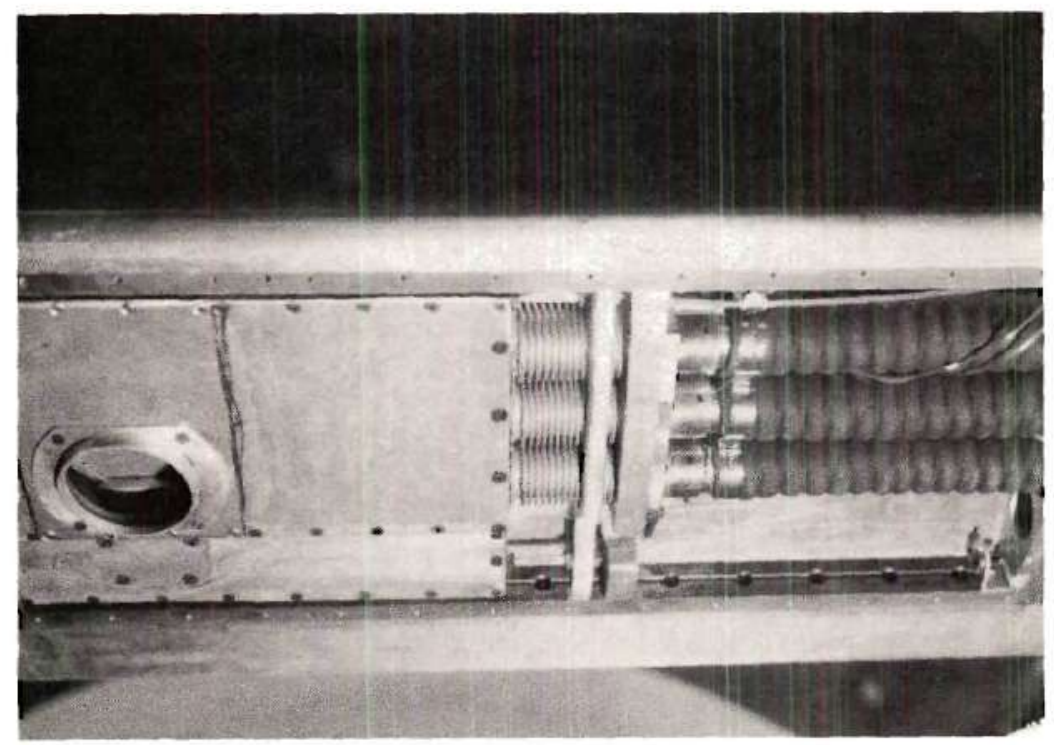

Figure 11. The Installed Plenum Chamber

(Wing Panel Removed, View Looking Downstream).

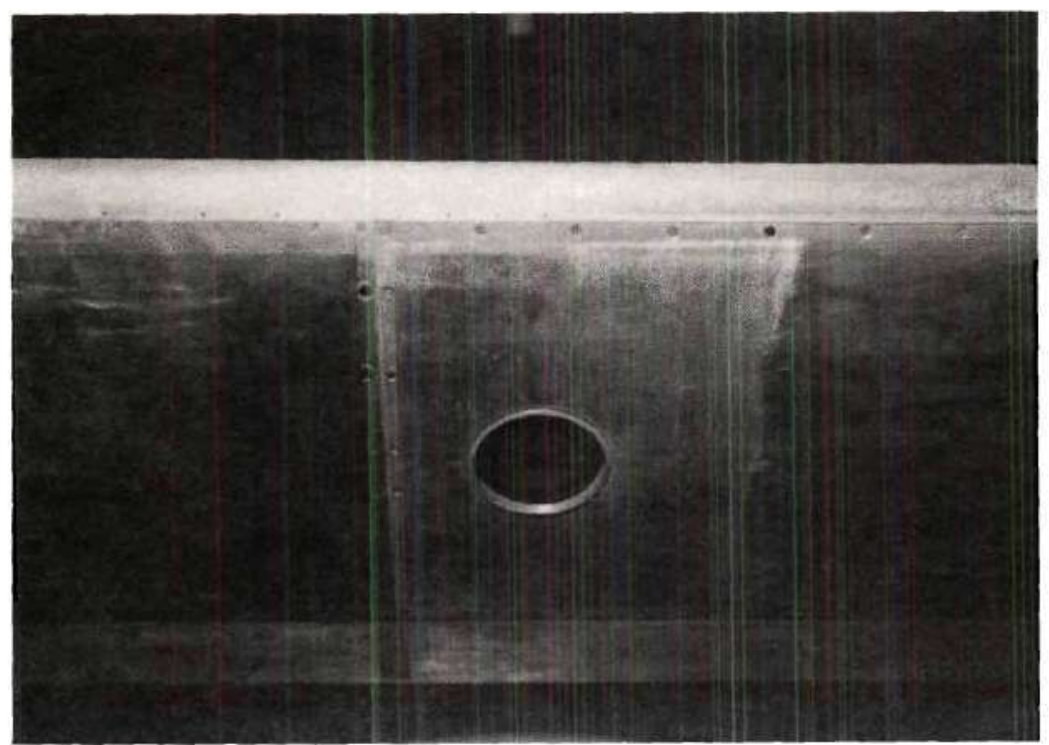

Figure 12. Wing Lower Surface with Three Inch Diameter Jet Installed

(View Looking Downstream). 

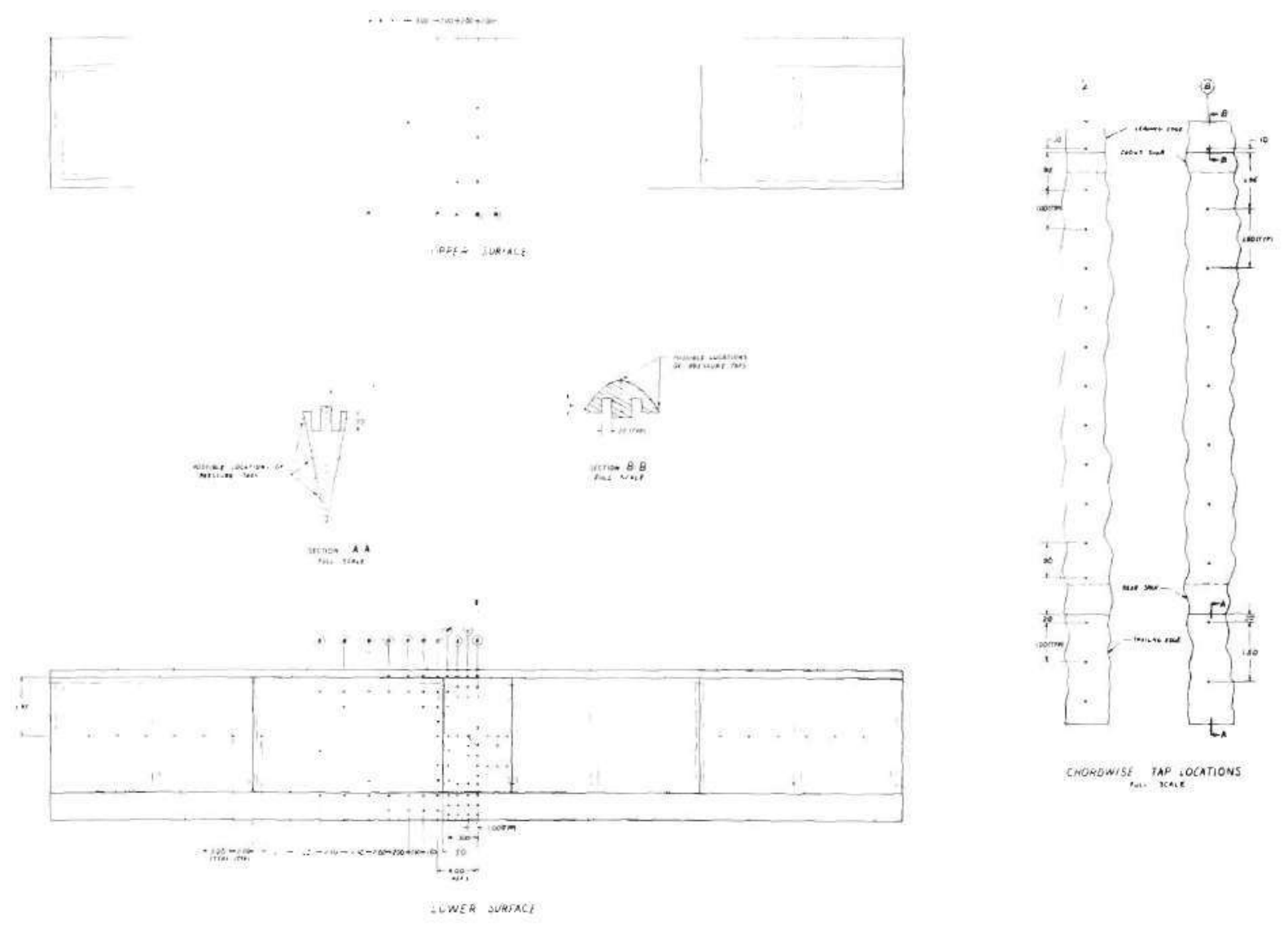

Figure 13. Location of Surface Pressure Taps. 


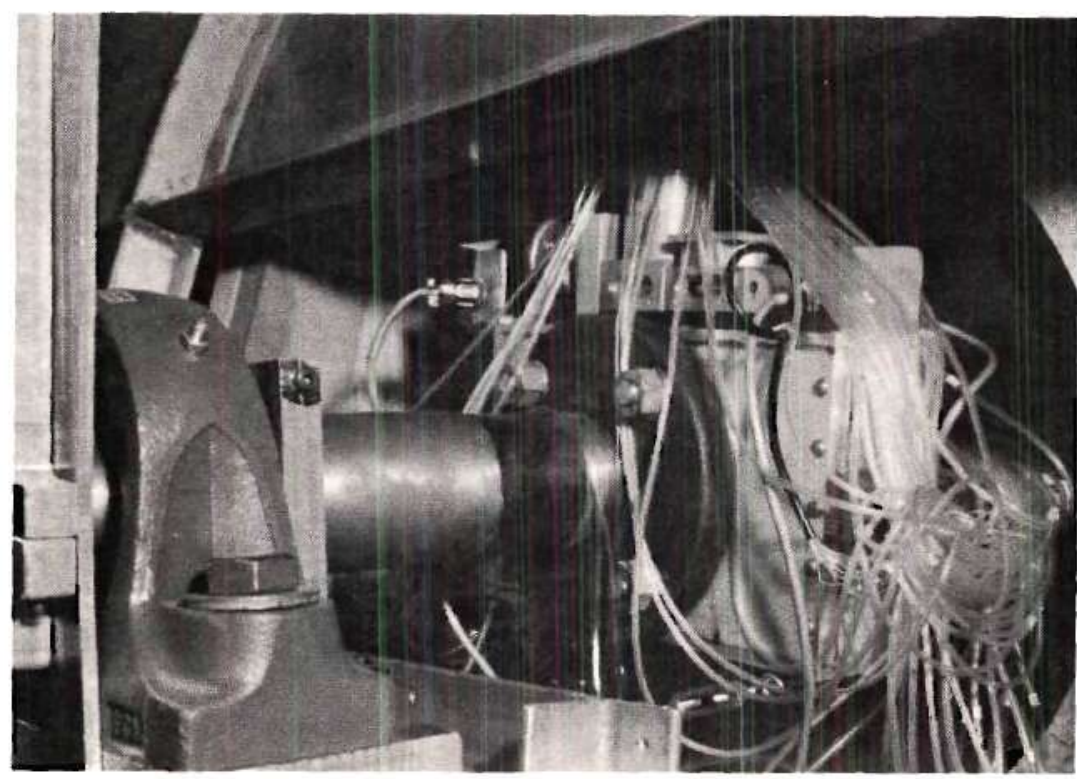

Figure 14. Pressure Lines and Scanivalve Mounted on External Plenum Chamber.

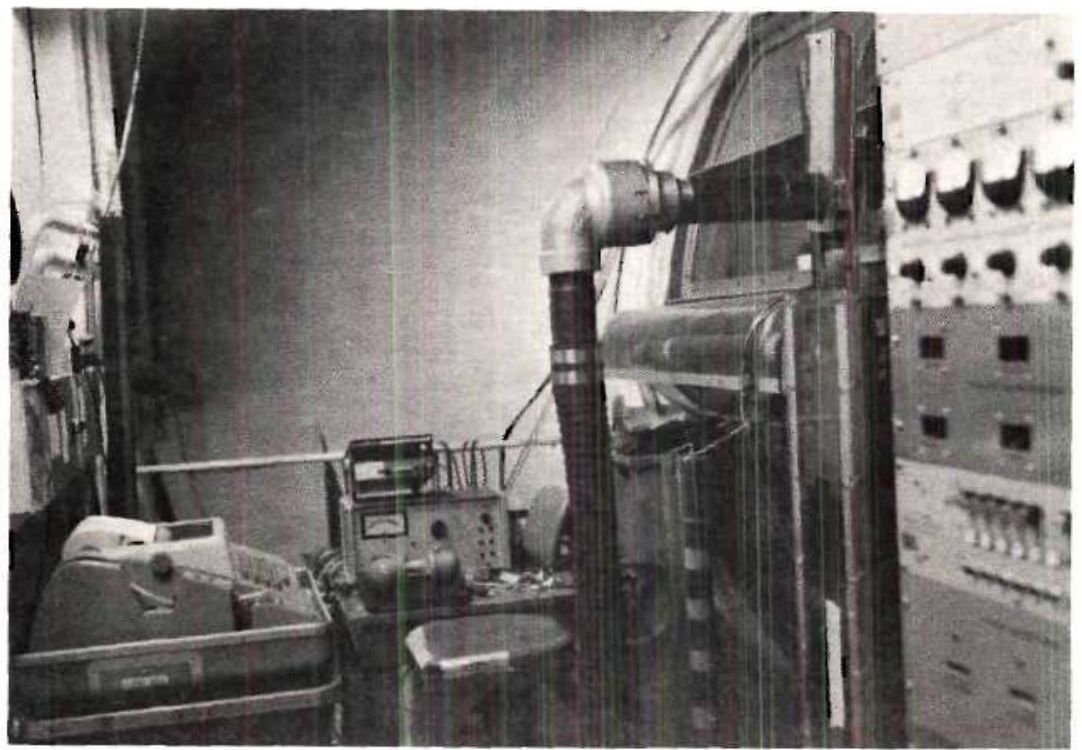

Figure 15. Operator's Area and Pressure Measurement Equipment. 


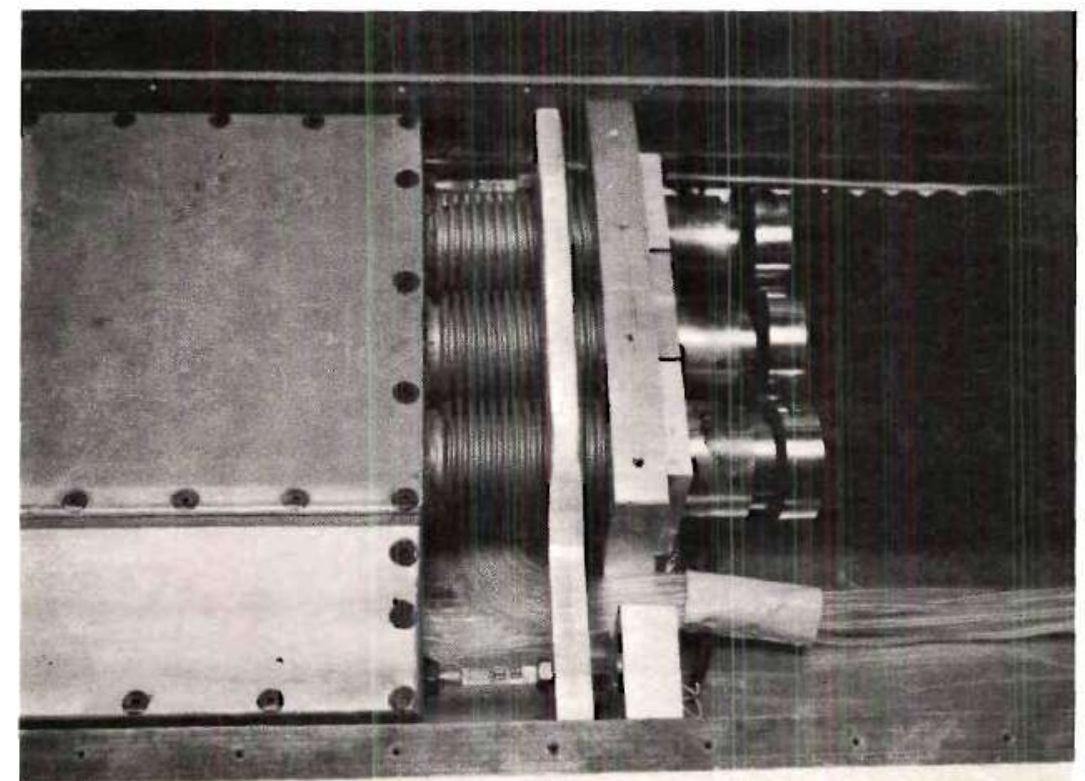

Figure 16. Plenum Chamber Attachment to Floating Rib on Flexures.

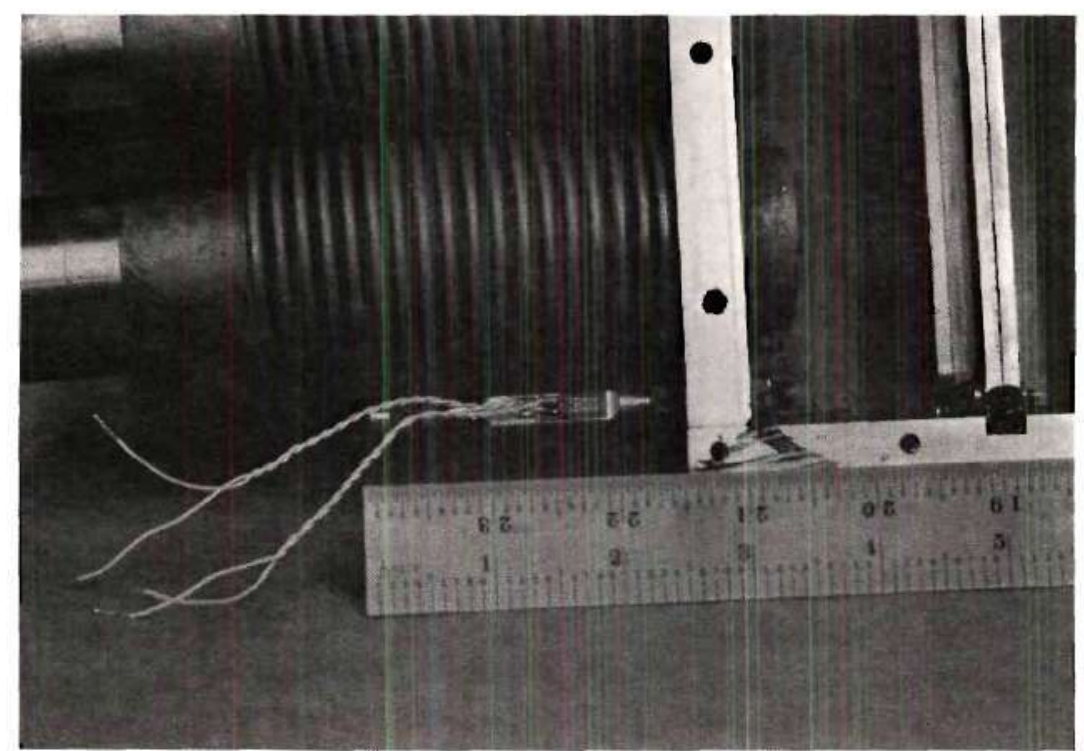

Figure 17. Strain Gages Mounted on Flexures. 


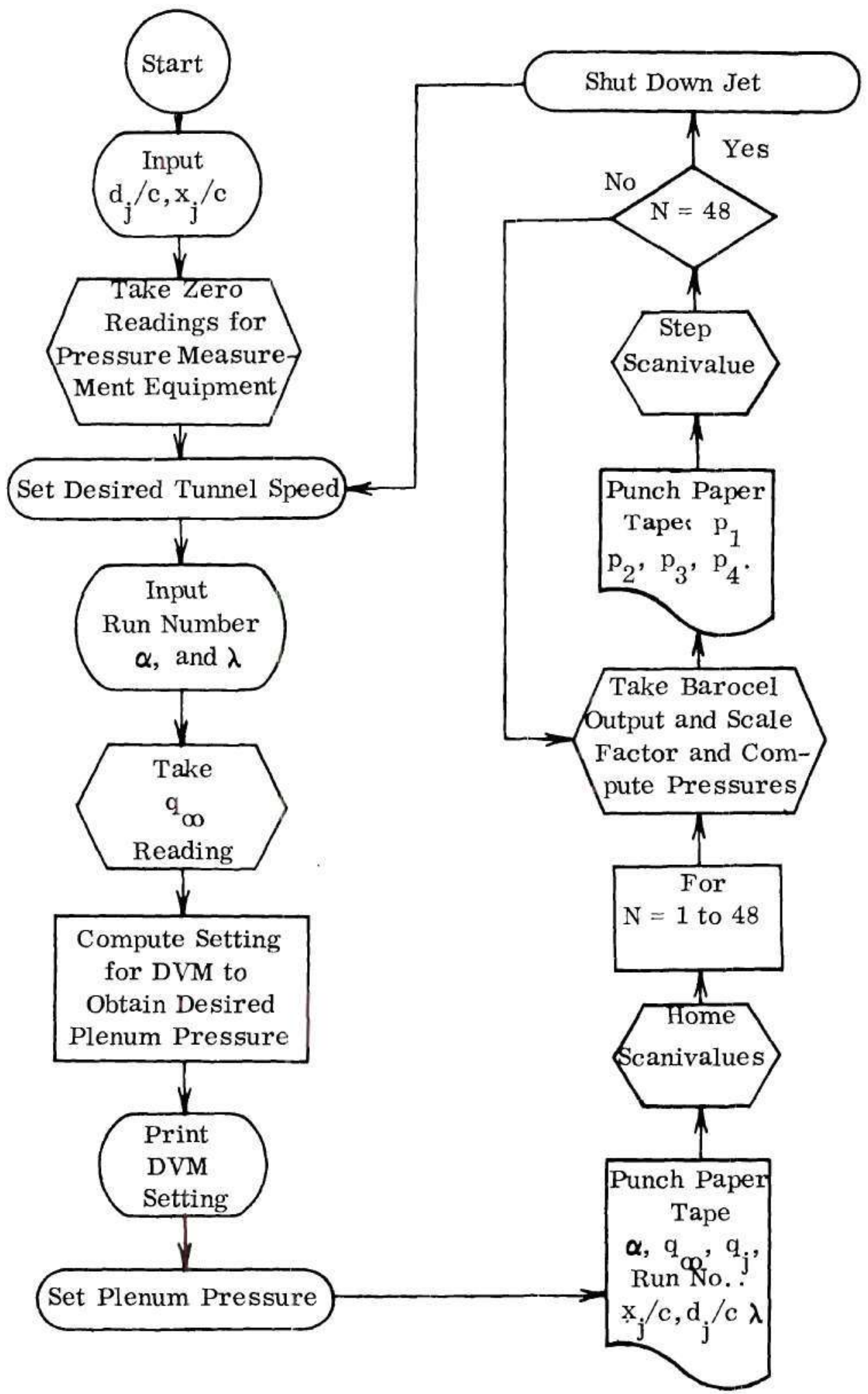

Figure 18. Automated Procedure for Acquiring Surface Pressures. 

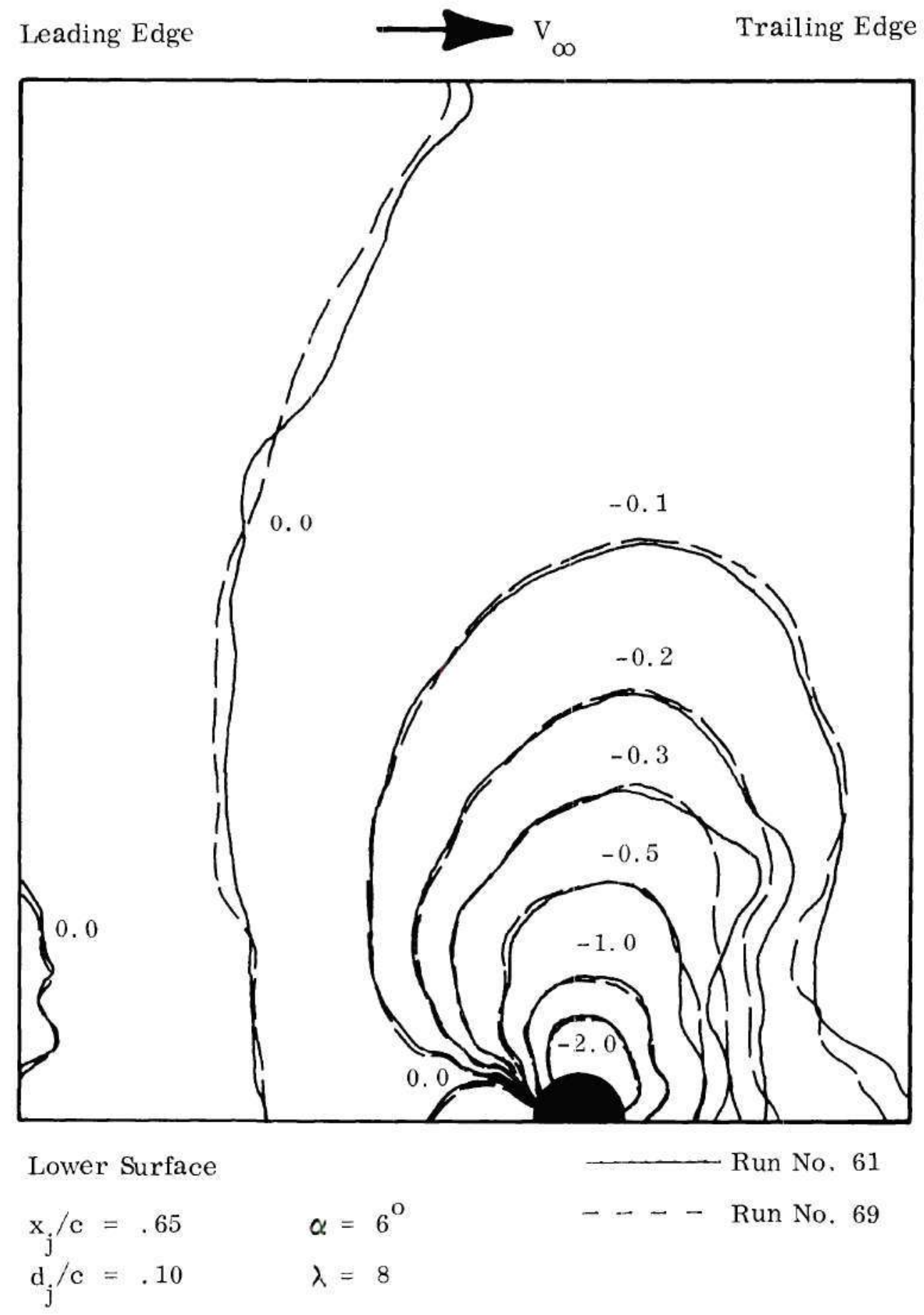

Figure 19. Repeatability of Interference Surface

Pressure Contours. 

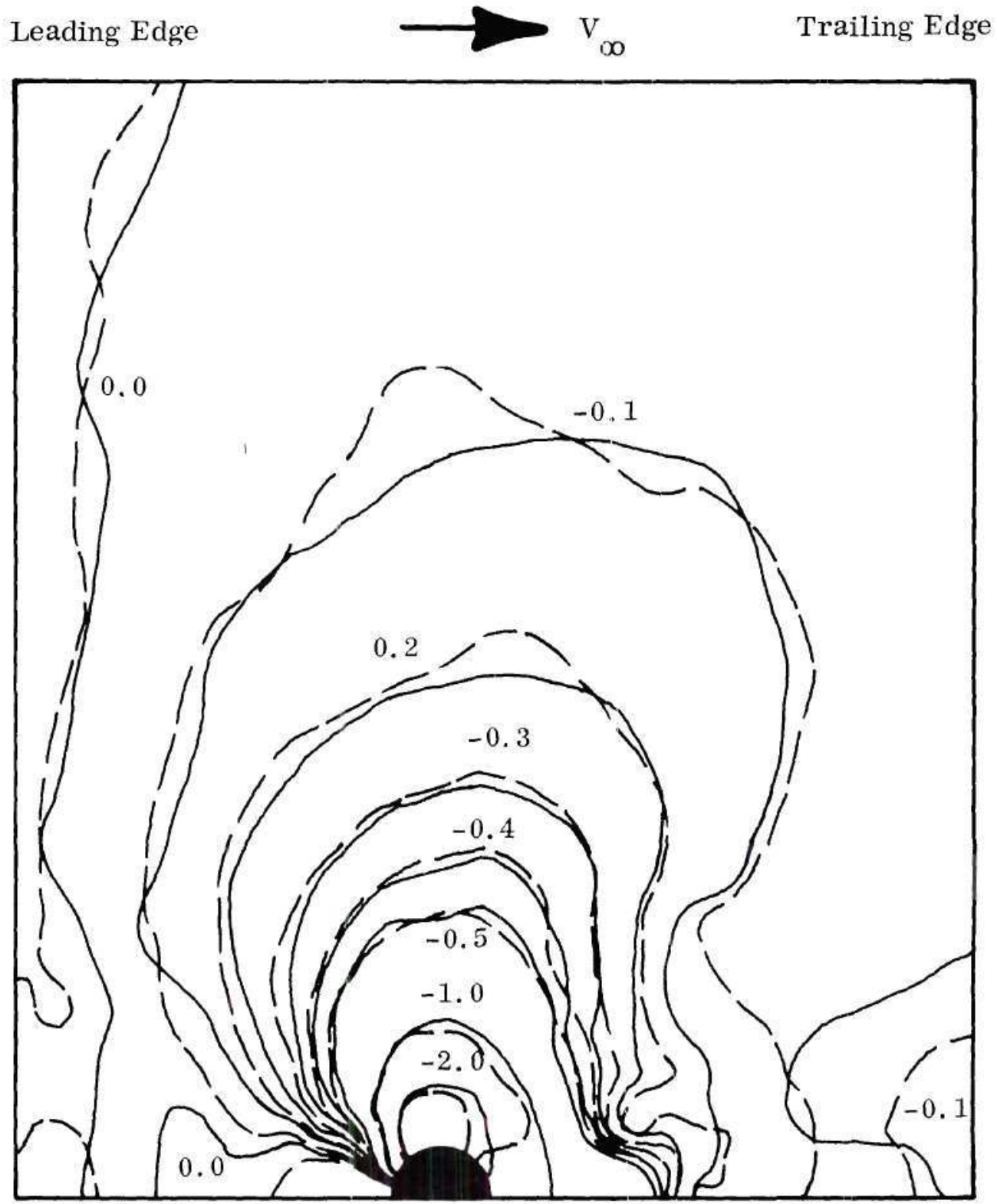

Lower Surface
$x_{j} / c=.45$
$\alpha=0^{\circ}$
$-\mathrm{V}_{\mathrm{i}}=100 \mathrm{Ft} / \mathrm{Sec}$
$\mathrm{d}_{\mathrm{j}} / \mathrm{c}=.10 \quad \lambda=8$
$-\cdots-\mathrm{V}_{\mathrm{i}}=50 \mathrm{Ft} / \mathrm{Sec}$

Figure 20. Interference Surface Pressure Contours for Different Freestream Velocities. 


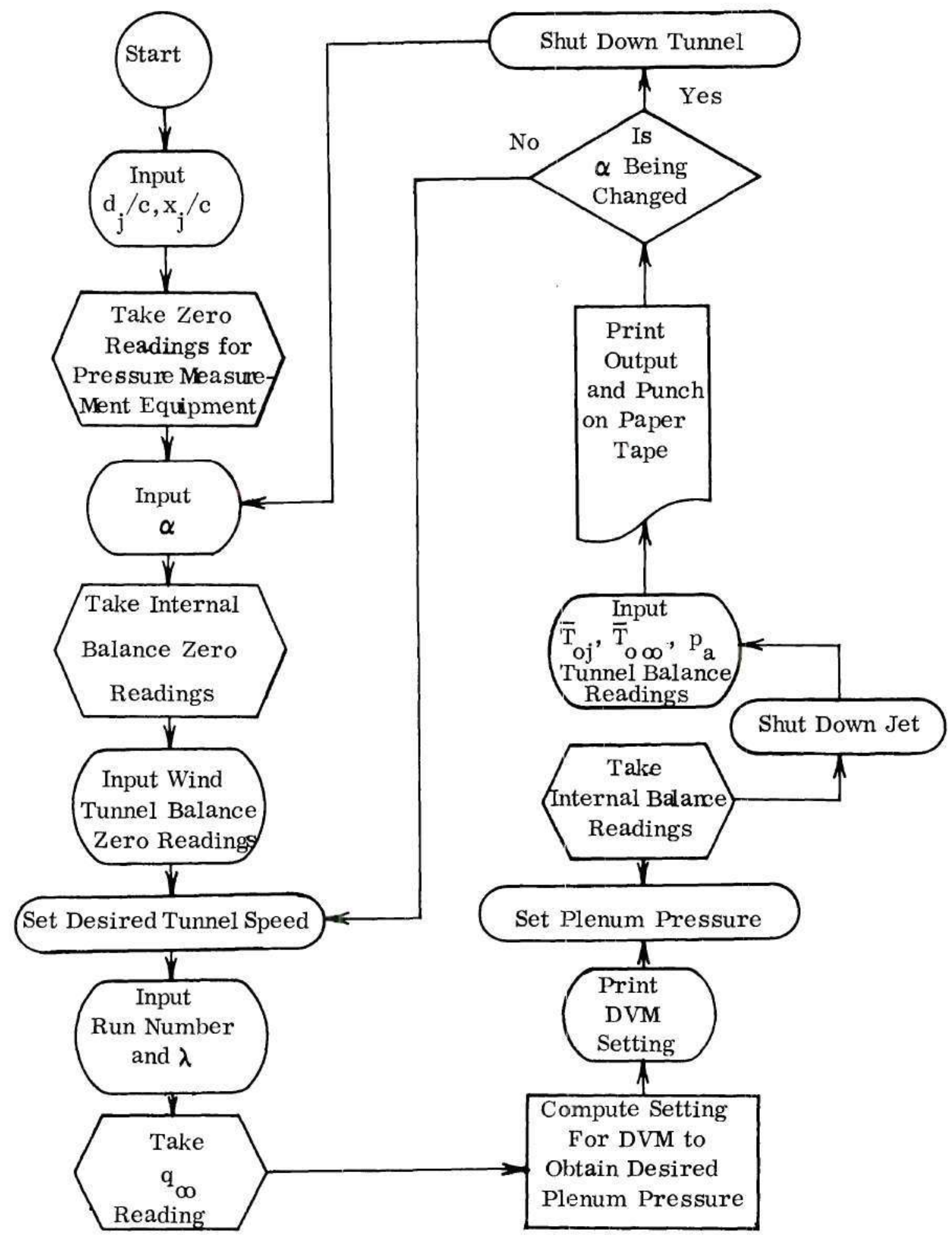

Figure 21. Automated Procedure for Acquiring Force Data. 


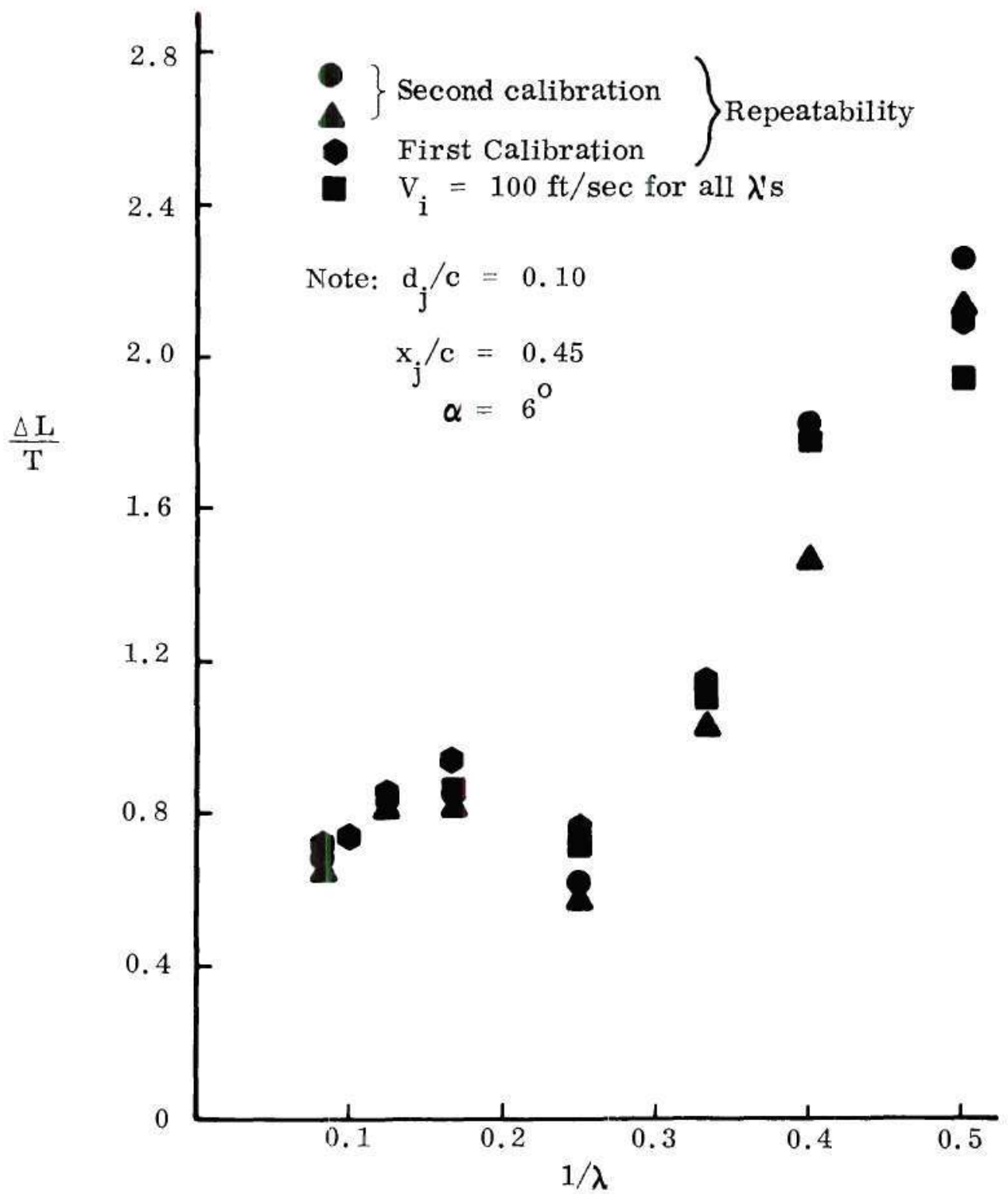

Figure 22. Repeatability of the Interference

Lift Coefficient Data. 


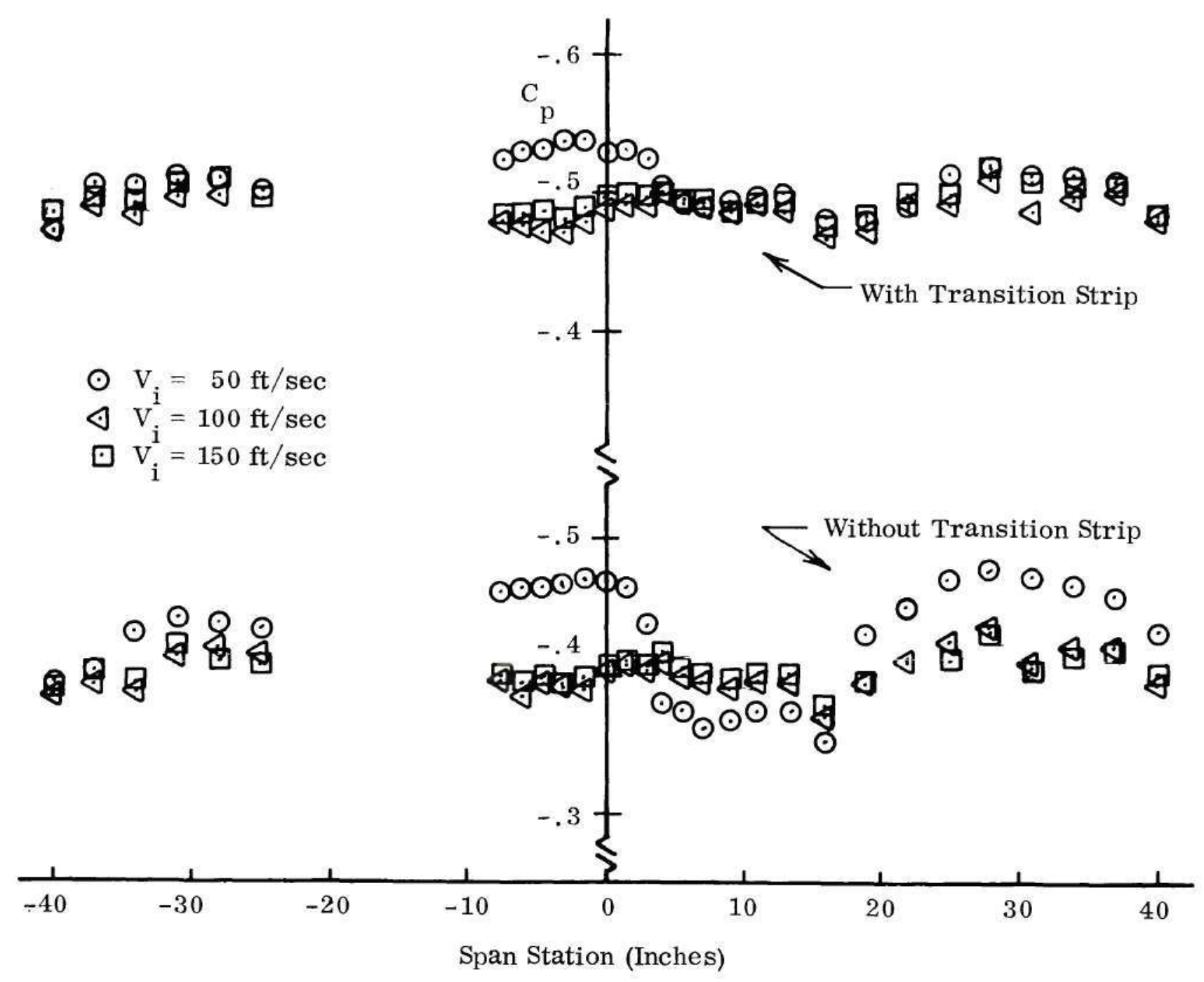

Figure 23. Lower Surface Spanwise Pressure Distributions for $\alpha=0^{\circ}$ at Chord Station 6.75 Inches $(\mathrm{x} / \mathrm{c}=.45)$ Showing Effect of Transition Strip. 


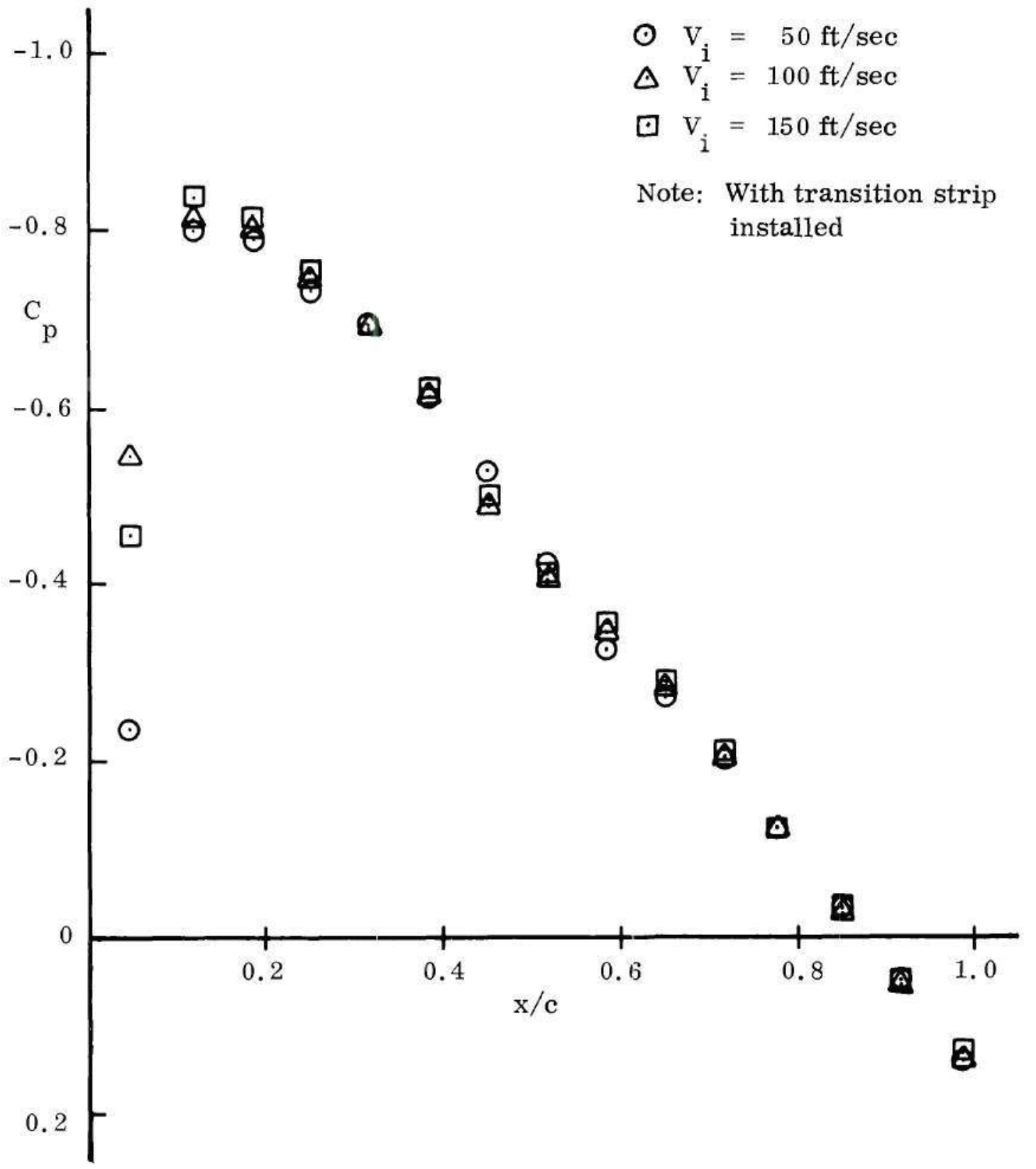

Figure 24. Lower Surface Chordwise Pressure Distributions for $\alpha=0^{\circ}$ at Wing Centerline for Different Tunnel Speeds. 


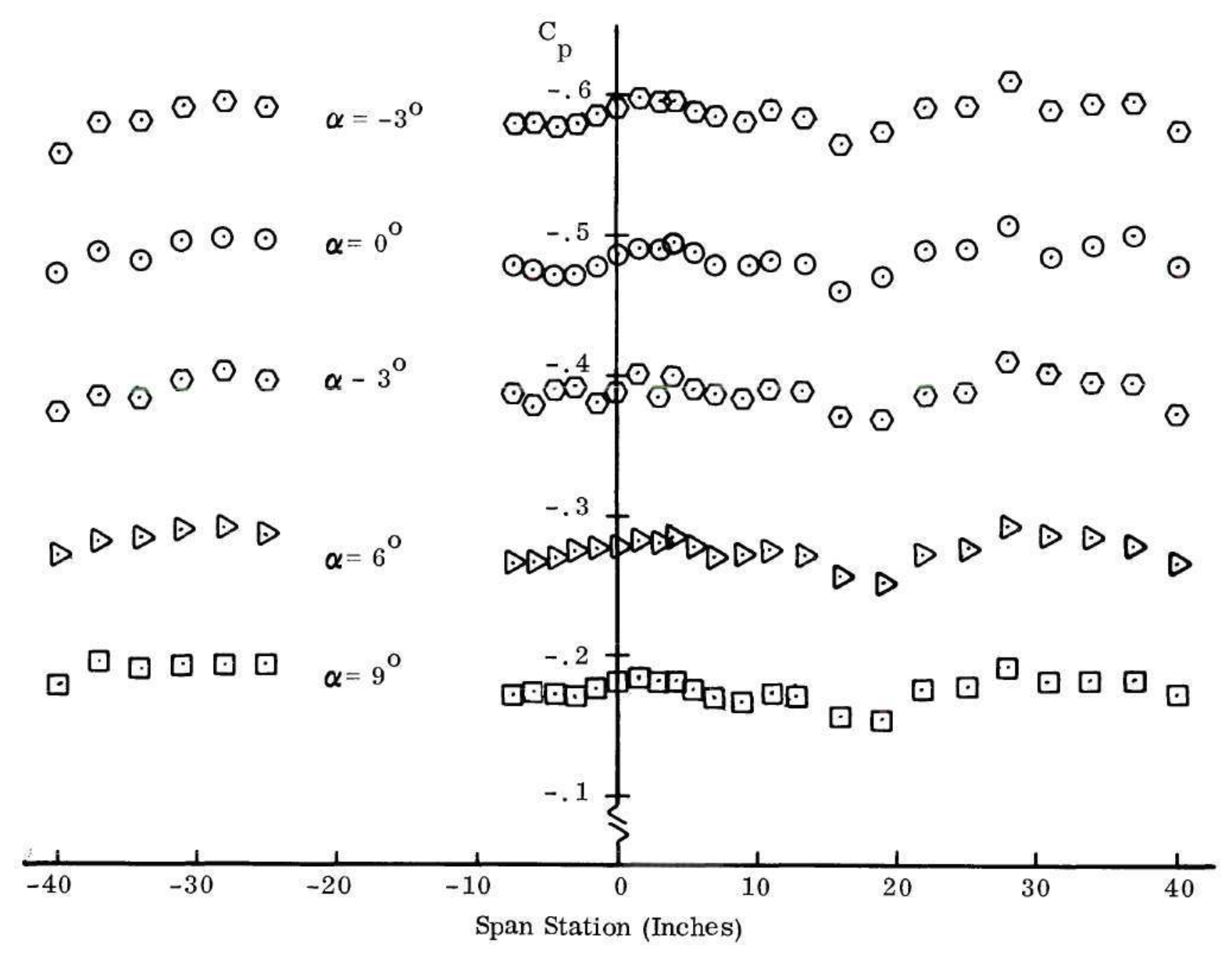

Figure 25. Lower Surface Spanwise Pressure Distributions at Chord Station

6.75 Inches $(x / c=.45)$ for $v_{i}=100 \mathrm{ft} / \mathrm{sec}$. 


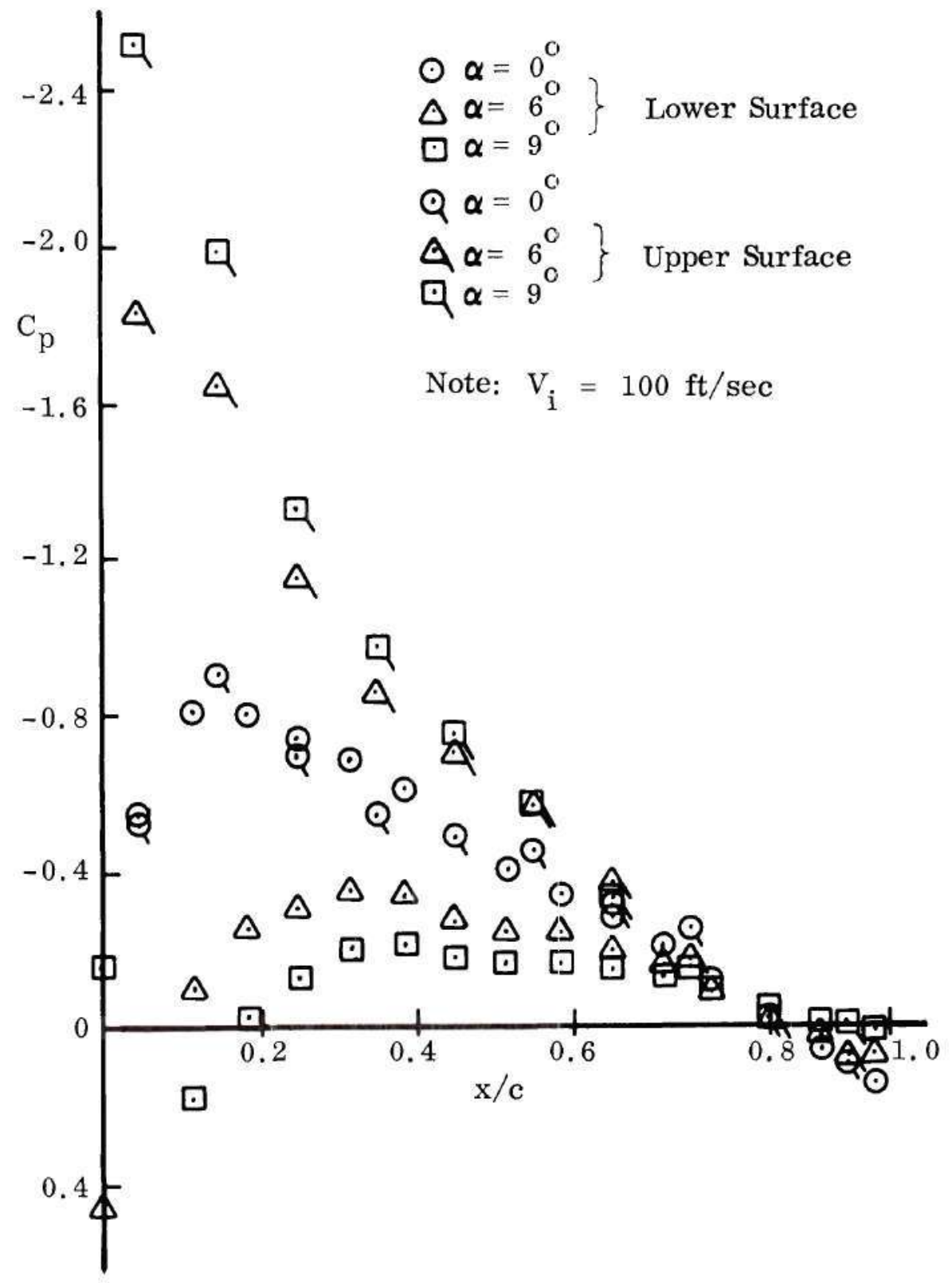

Figure 26. Jet-off Chordwise Pressure Distributions at Wing Centerline for Different Angles of Attack. 


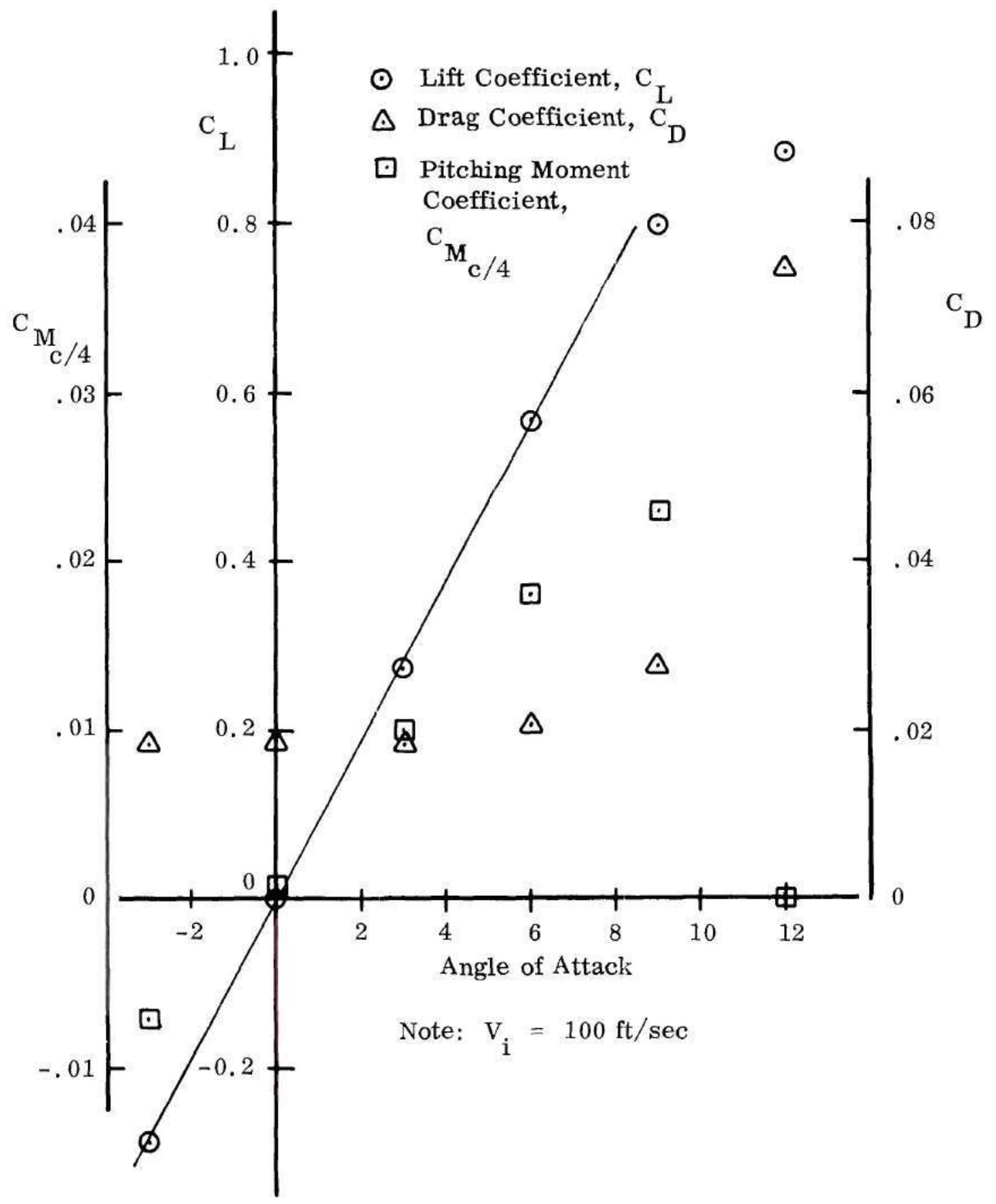

Figure 27. Basic Wing Aerodynamic Coefficients for the Model. 


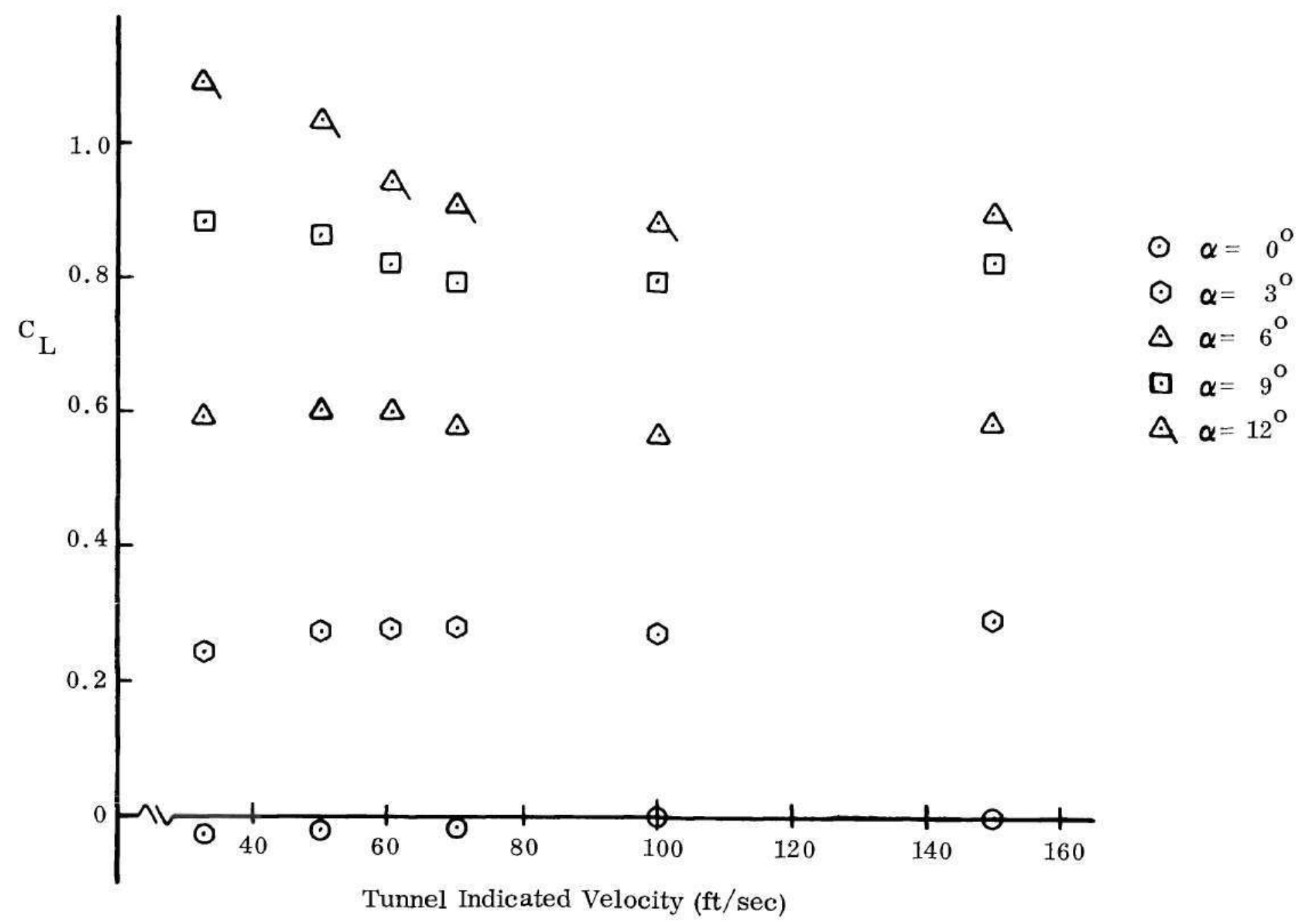

Figure 28. Wing Lift Coefficients for Various Tunnel Speeds. 


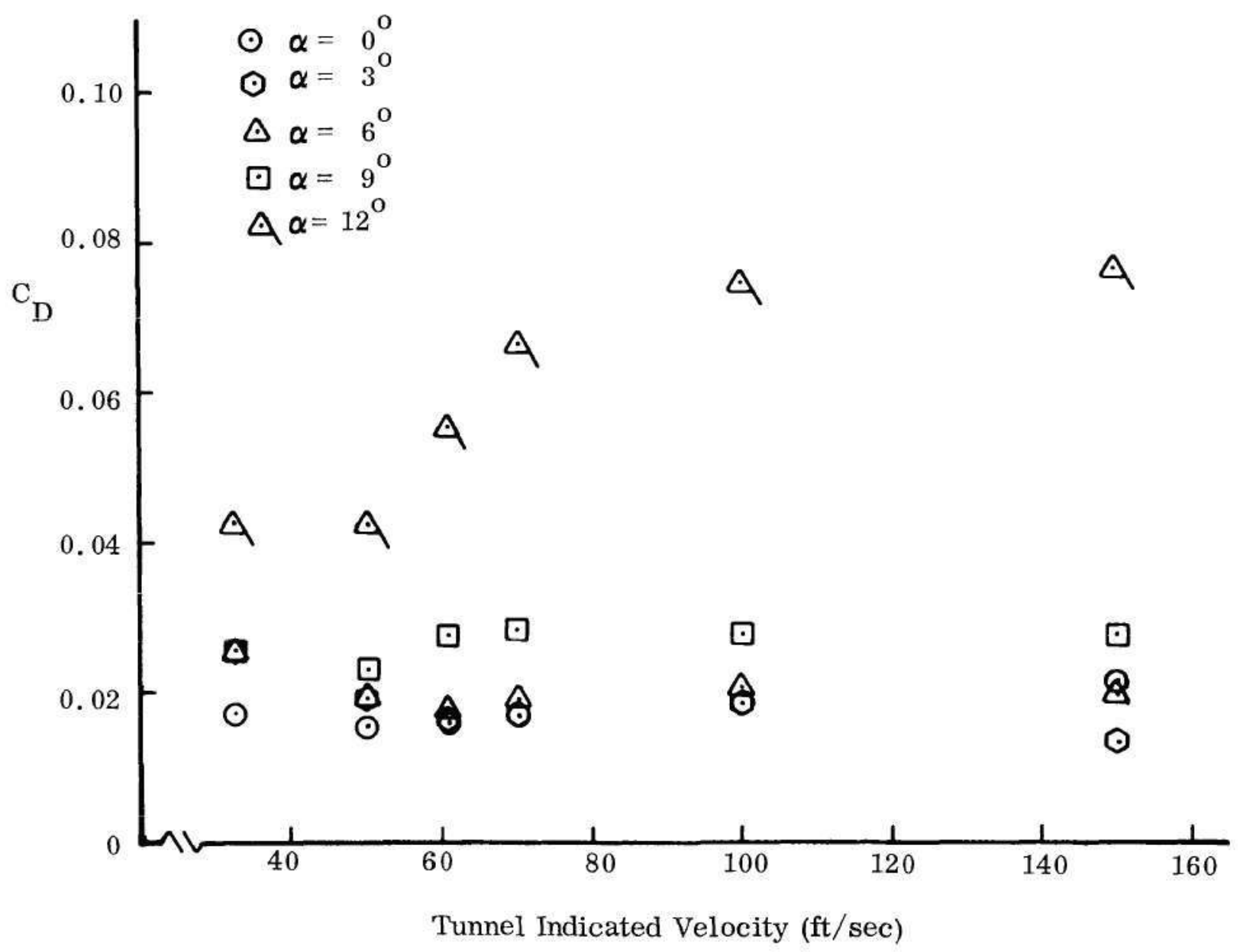

Figure 29. Wing Drag Coefficient for Various Tunnel Speeds. 


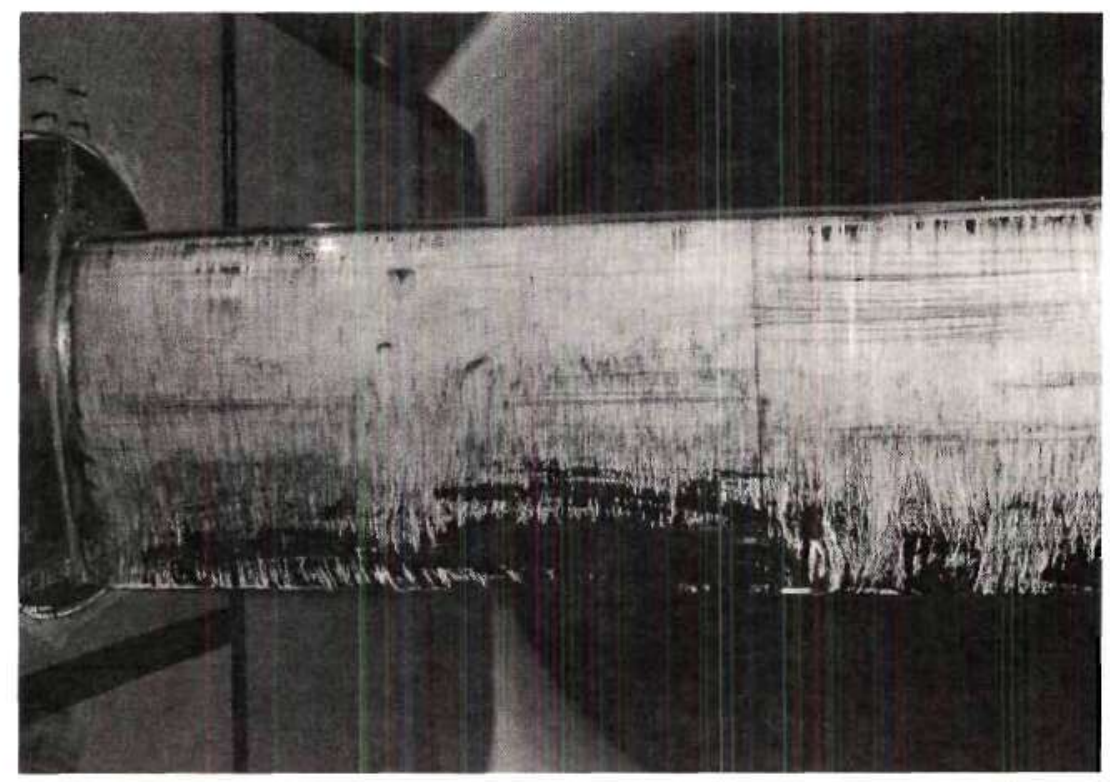

(a) $\mathrm{V}_{\mathrm{i}}=70 \mathrm{Ft} / \mathrm{Sec}$

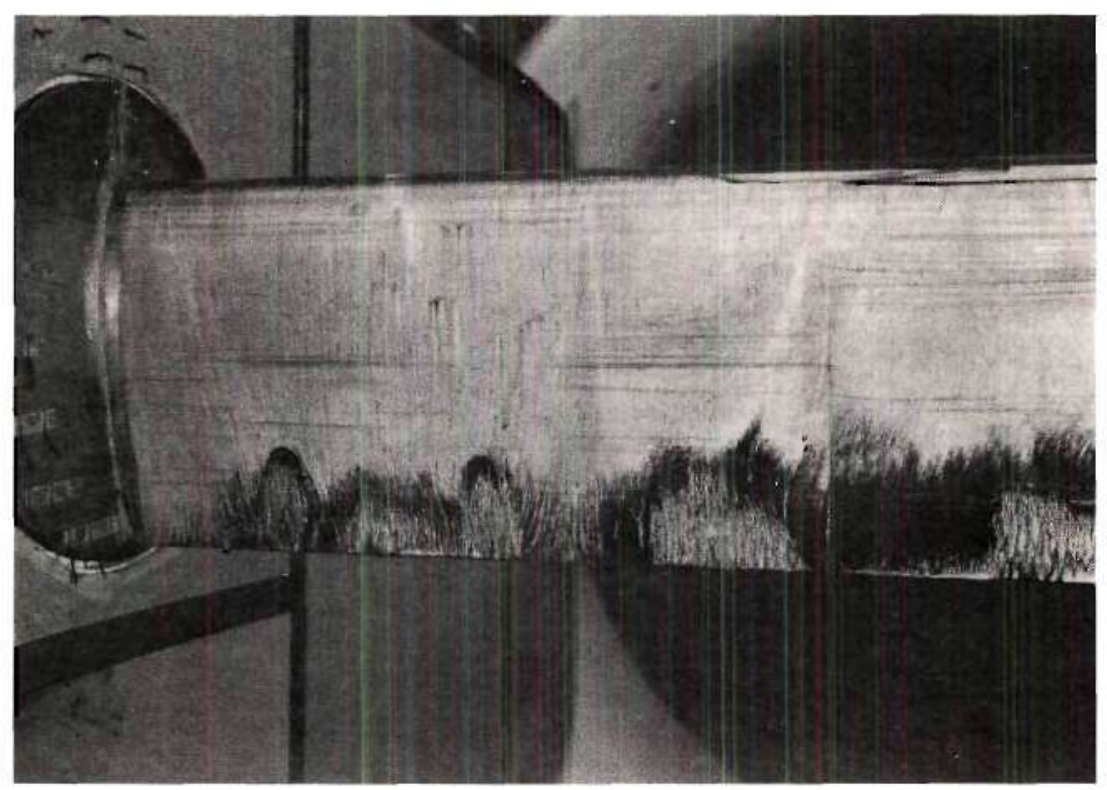

(b) $V_{i}=150 \mathrm{Ft} / \mathrm{Sec}$

Figure 30. Oil Flow on Upper Surface at $\alpha=9^{\circ}$ and $\lambda=0$. 


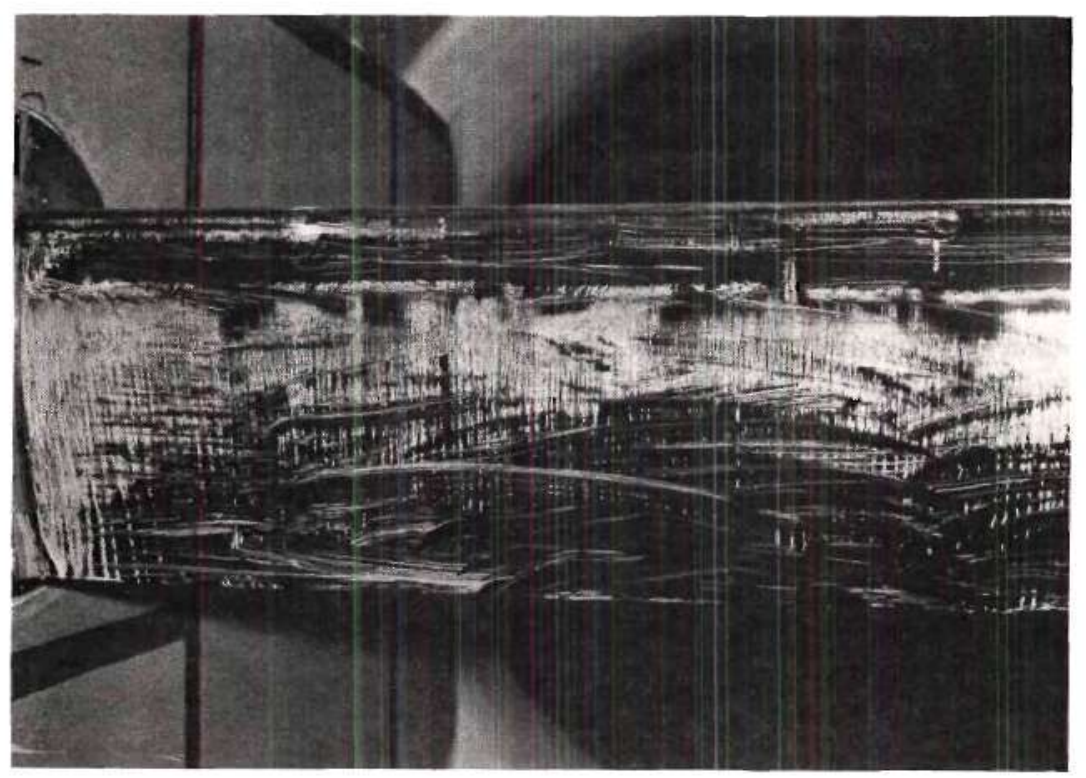

(a) $\mathrm{V}_{\mathrm{i}}=32.8 \mathrm{Ft} / \mathrm{Sec}, \lambda=0$

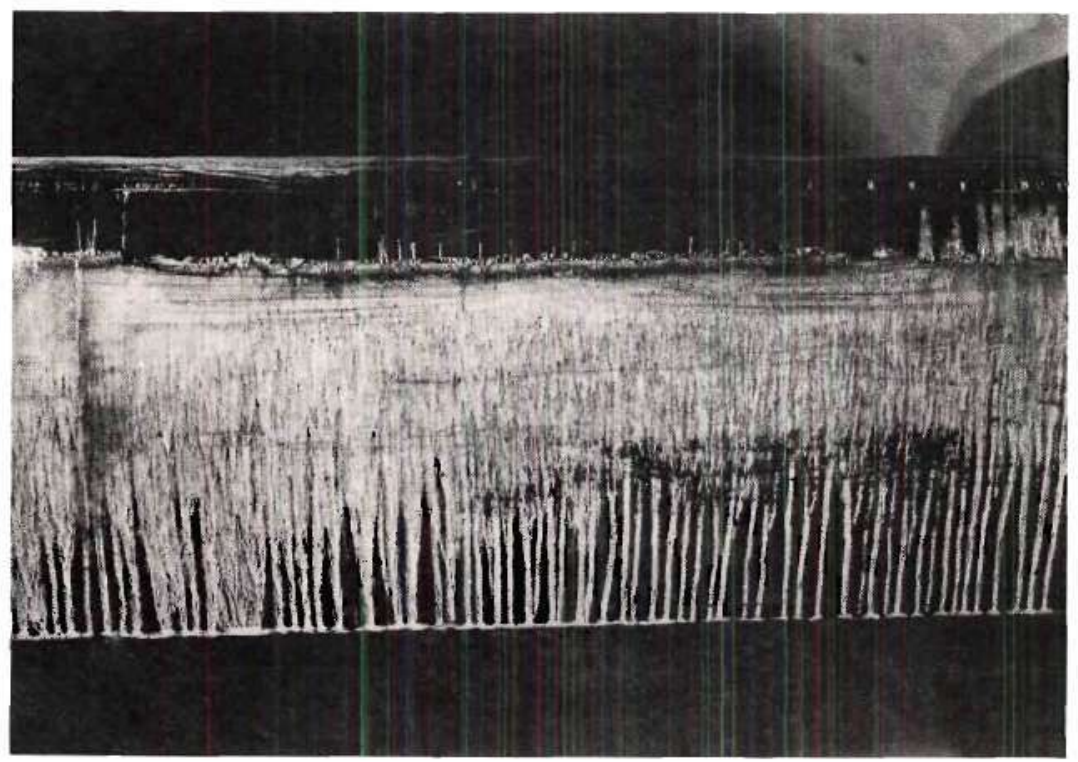

(b) $\mathrm{V}_{\mathrm{i}}=32.8 \mathrm{Ft} / \mathrm{Sec}, \lambda=8$

Figure 31. Oil Flow Exhibiting Laminar Separation Bubble on the Upper Surface at $\alpha=9^{\circ}$. 


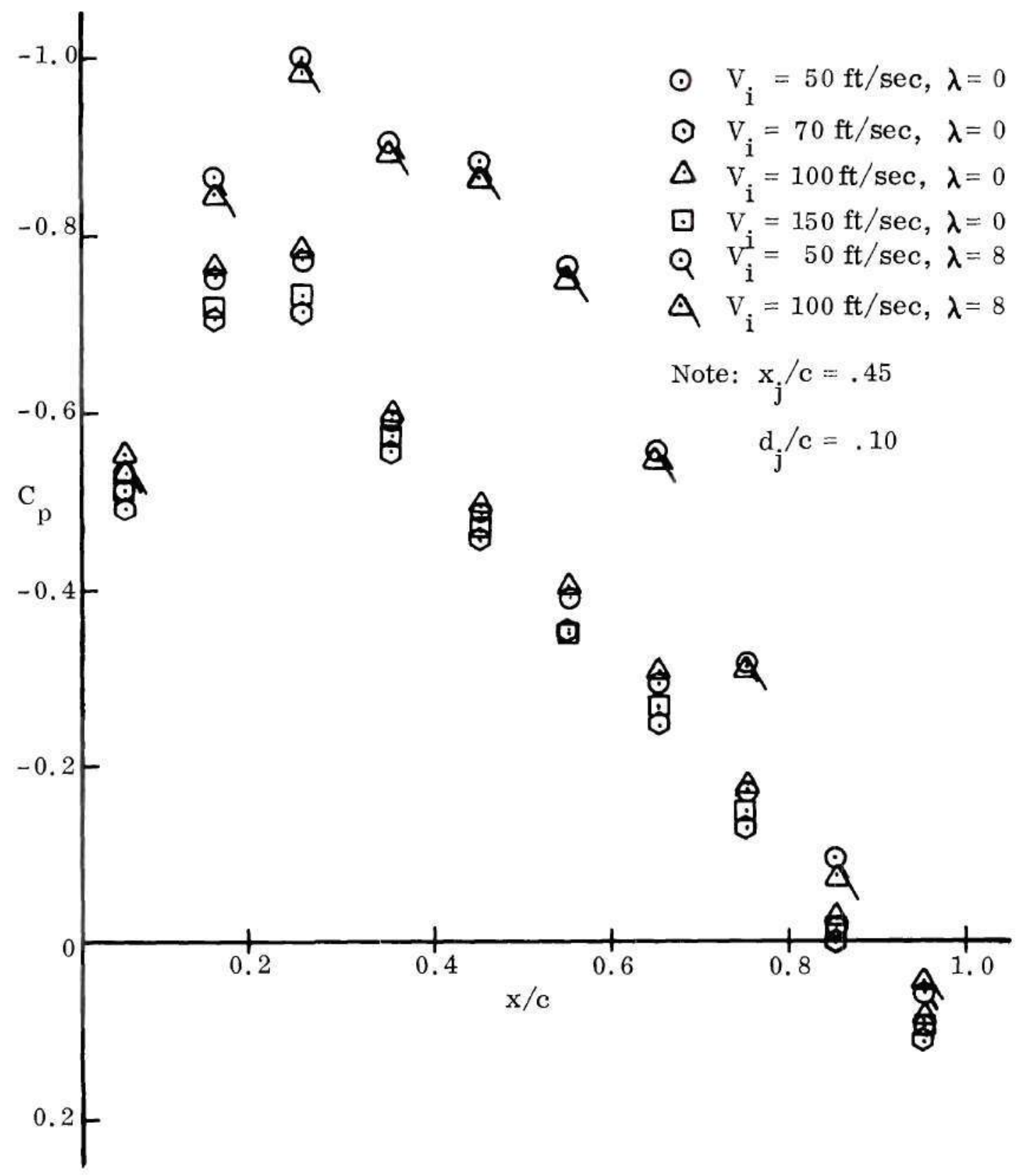

Figure 32. Lower Surface Chordwise Pressure Distributions at Span Station 5.50 Inches for $\alpha=0^{\circ}$ with and without Jet Operation. 


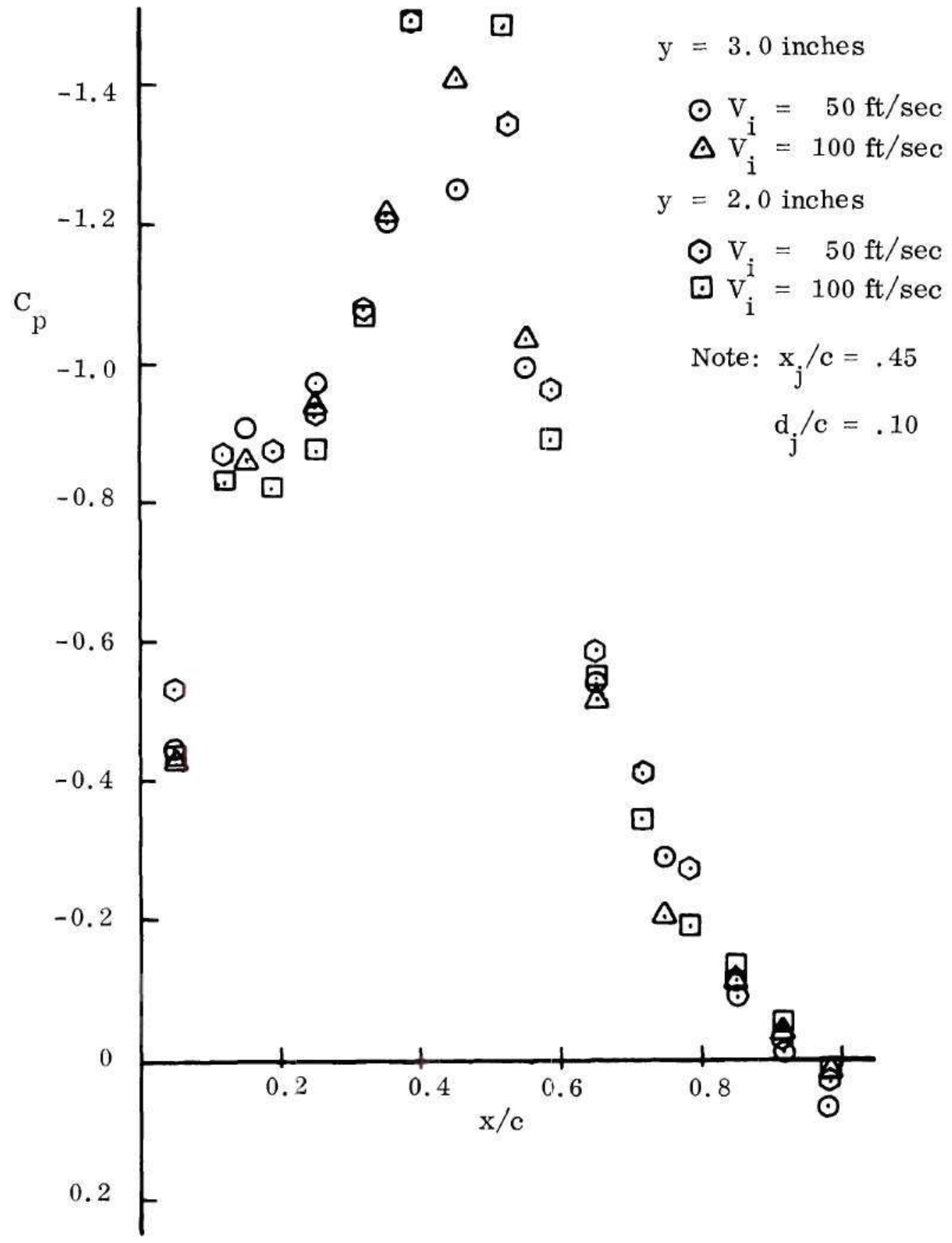

Figure 33. Lower Surface Chordwise Pressure Distributions

$$
\text { for } \boldsymbol{\alpha}=0^{\circ} \text { and } \lambda=8 \text {. }
$$




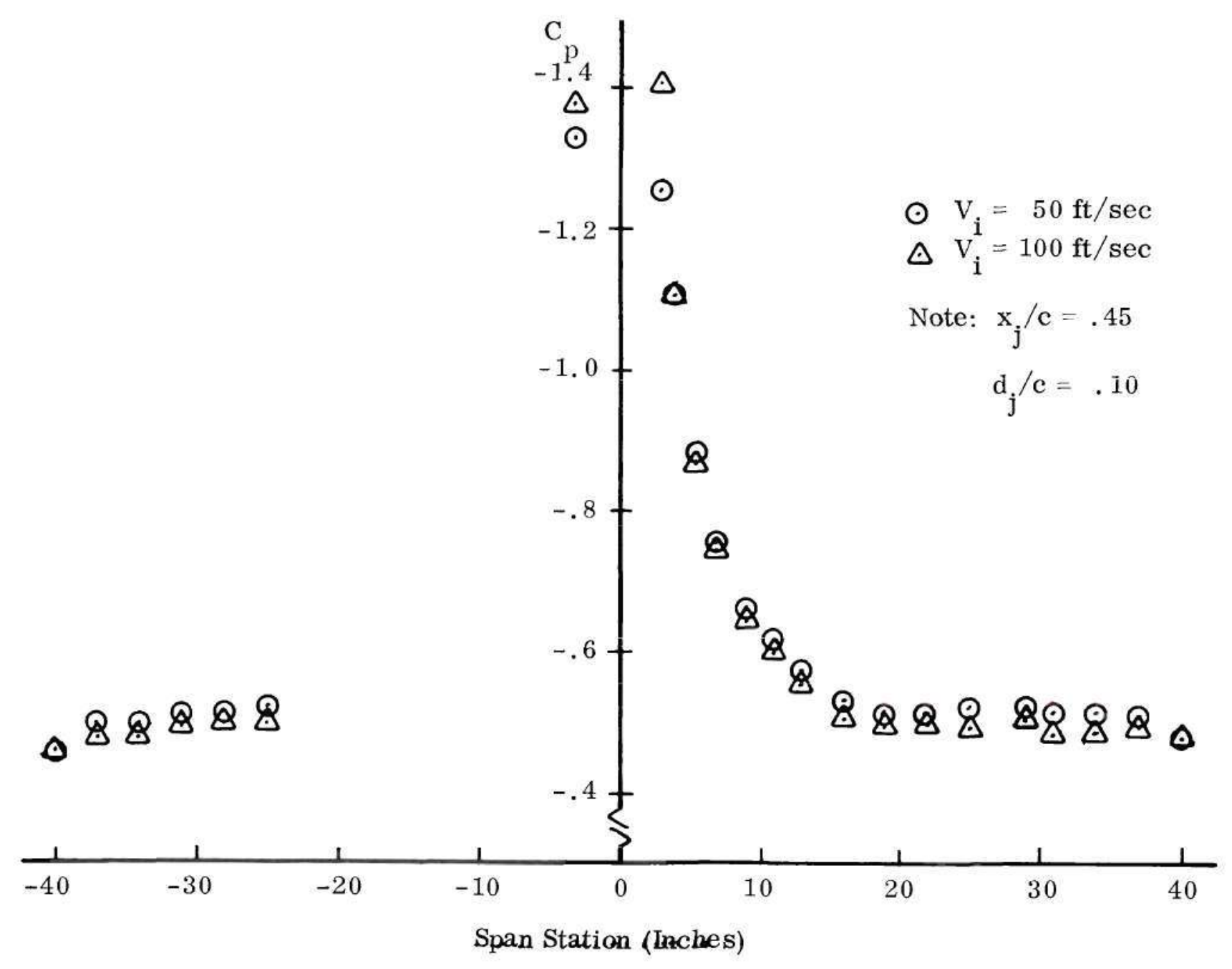

Figure 34. Lower Surface Spanwise Pressure Distributions at Chord Station

6.75 Inches $(\mathrm{x} / \mathrm{c}=0.45)$ for $\alpha=0^{\circ}$ and $\lambda=8$. 

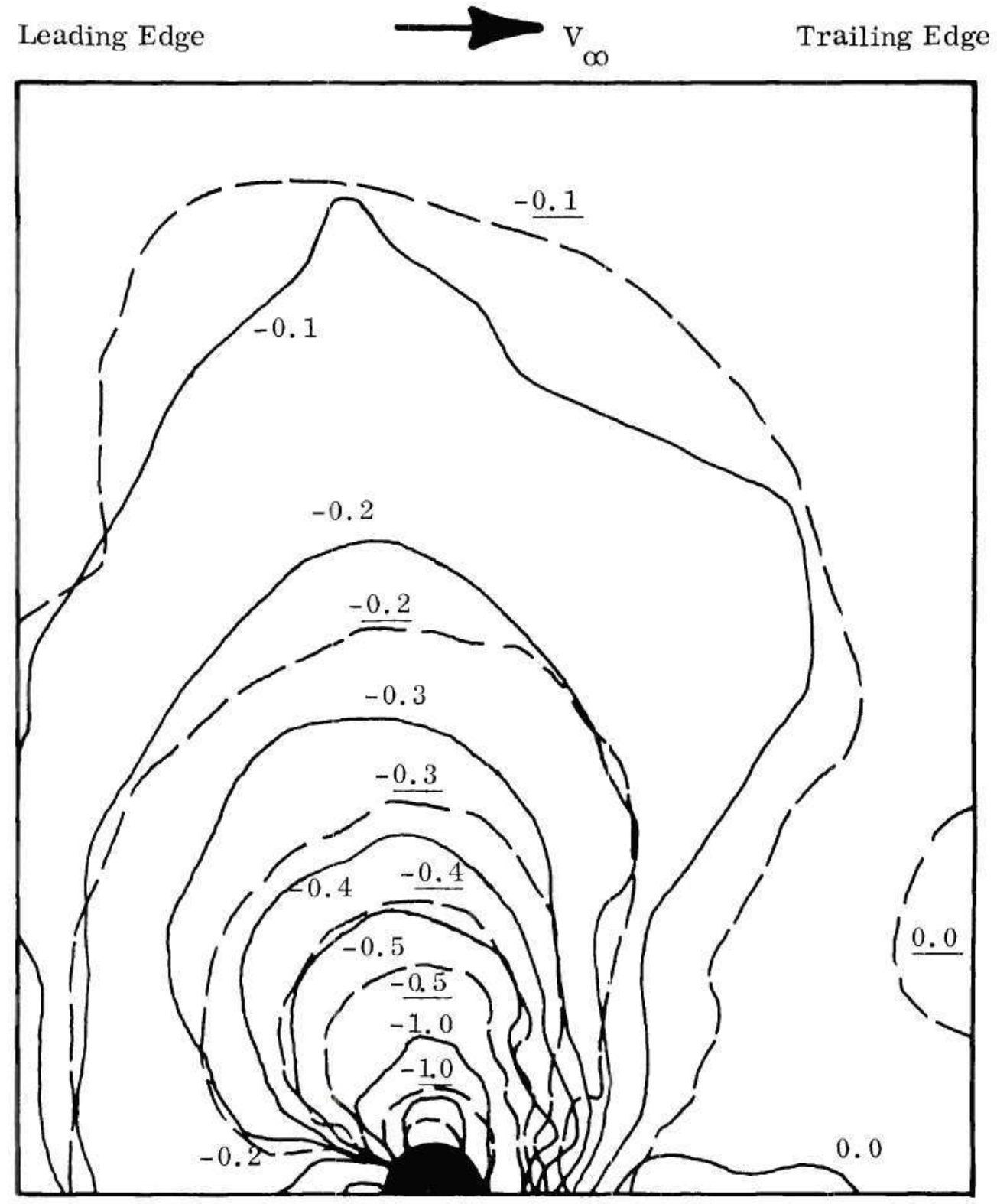

Lower Surface
$\mathrm{x}_{\mathrm{j}} / \mathrm{c}=.45$
$\alpha=0^{\mathrm{o}}$
$\longrightarrow \mathrm{C}_{\mathrm{p}} \longrightarrow$ Wing
$\mathrm{d}_{\mathrm{j}} / \mathrm{c}=.10$
$\lambda=12$
$-\mathrm{C}_{\mathrm{p}}---$ Flat Plate

Figure 35a. Interference Surface Pressure Contours for the Wing and for a Flat Plate. 

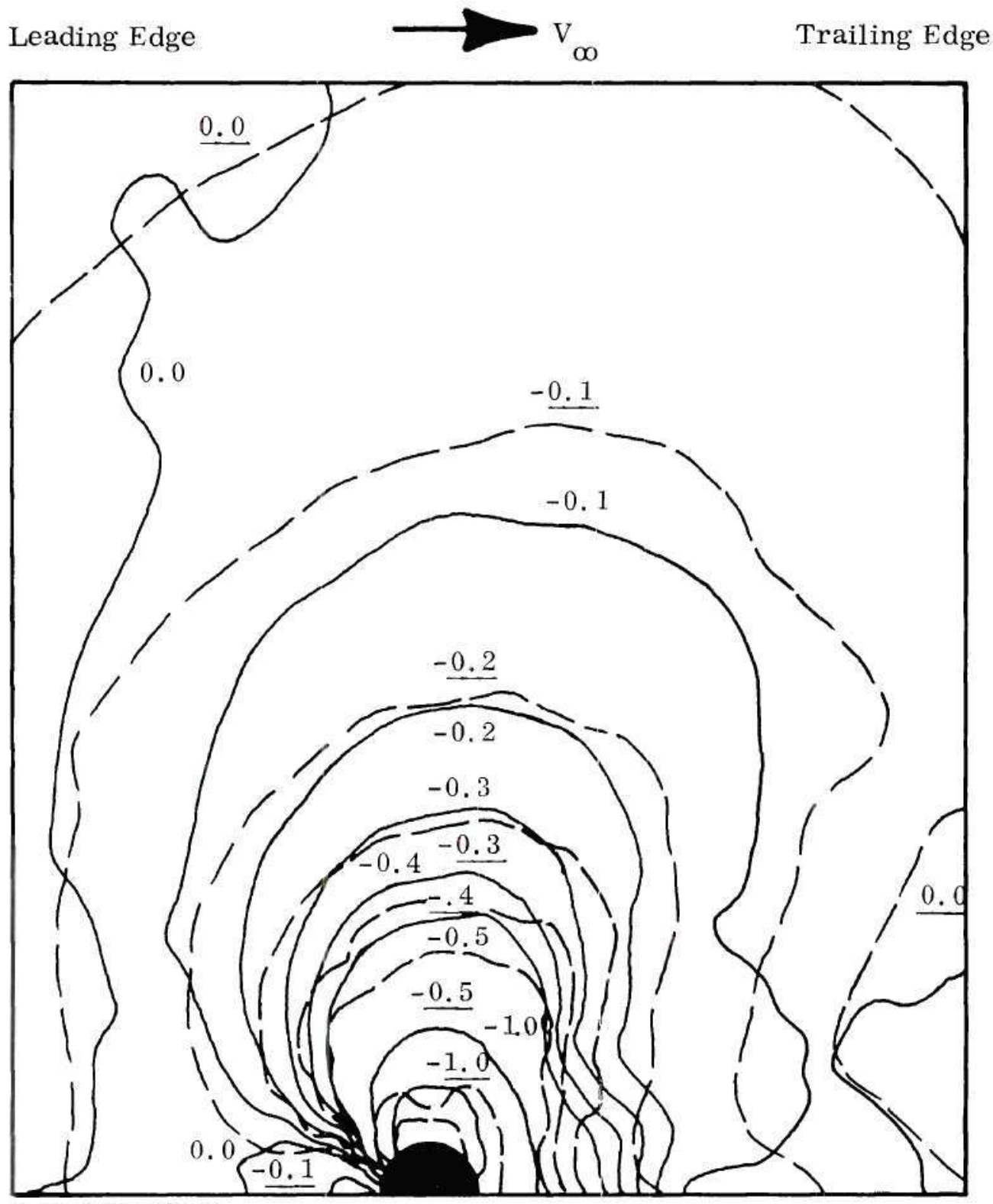

Lower Surface
$x_{j} / c=.45$
$\alpha=0^{\circ}$
$\longrightarrow \mathrm{C}_{\mathrm{p}} \longrightarrow$ Wing
$\mathrm{d}_{\mathrm{j}} / \mathrm{c}=.10$
$\lambda=8$
$-{ }_{\mathrm{p}}^{\mathrm{C}}-\cdots-$ Flat Plate

Figure 35b. Interference Surface Pressure Contours for the Wing and for A Flat Plate. 

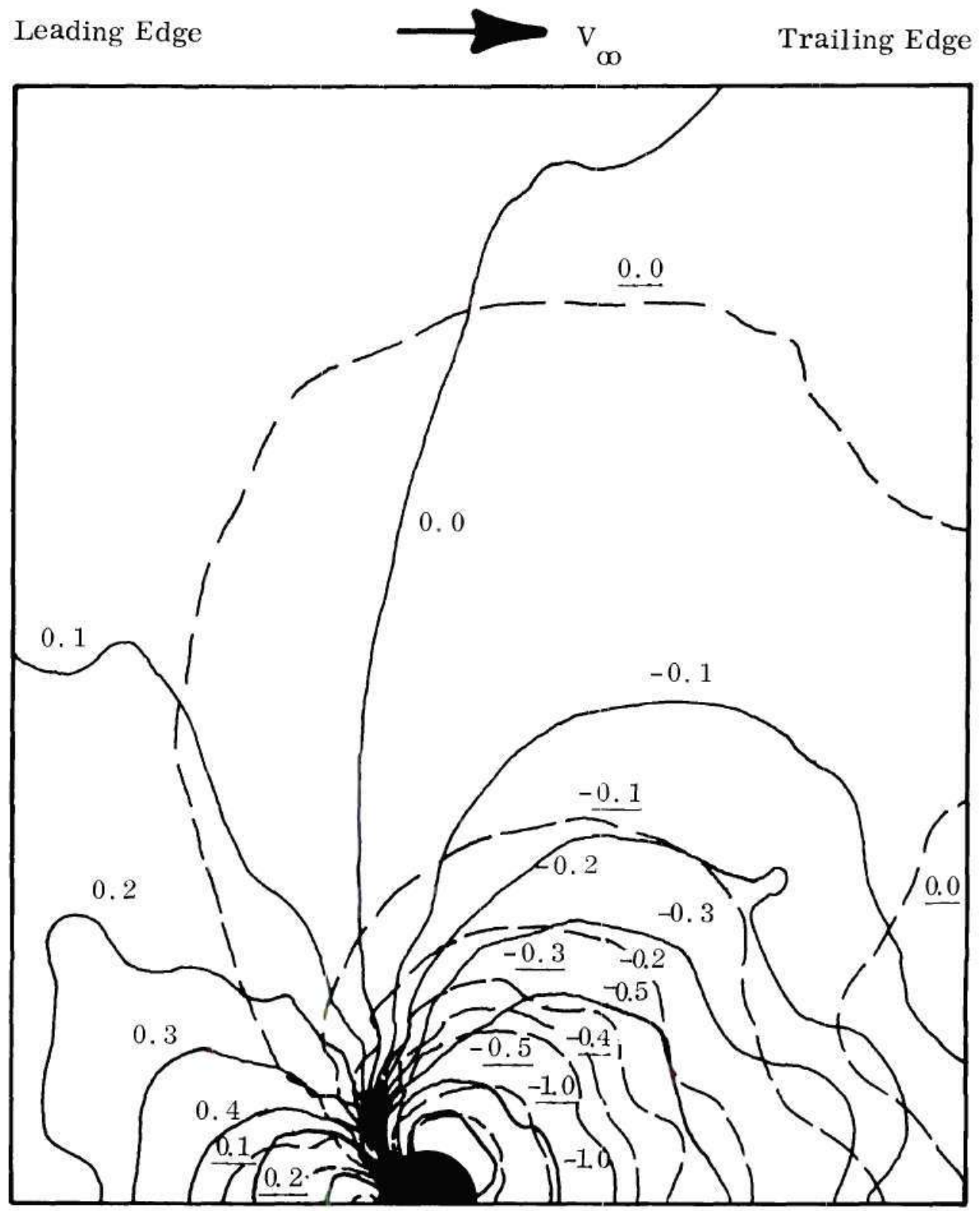

Lower Surface
$\mathrm{x}_{\mathrm{j}} / \mathrm{c}=.45$
$\alpha=0^{\mathrm{O}}$

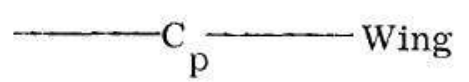
$\mathrm{d}_{\mathrm{j}} / \mathrm{c}=.10$
$\lambda=4$
$-\underset{\mathrm{p}}{-\mathrm{C}}---$ Flat Plate

Figure 35c. Interference Surface Pressure Contours for the Wing and for a Flat Plate. 


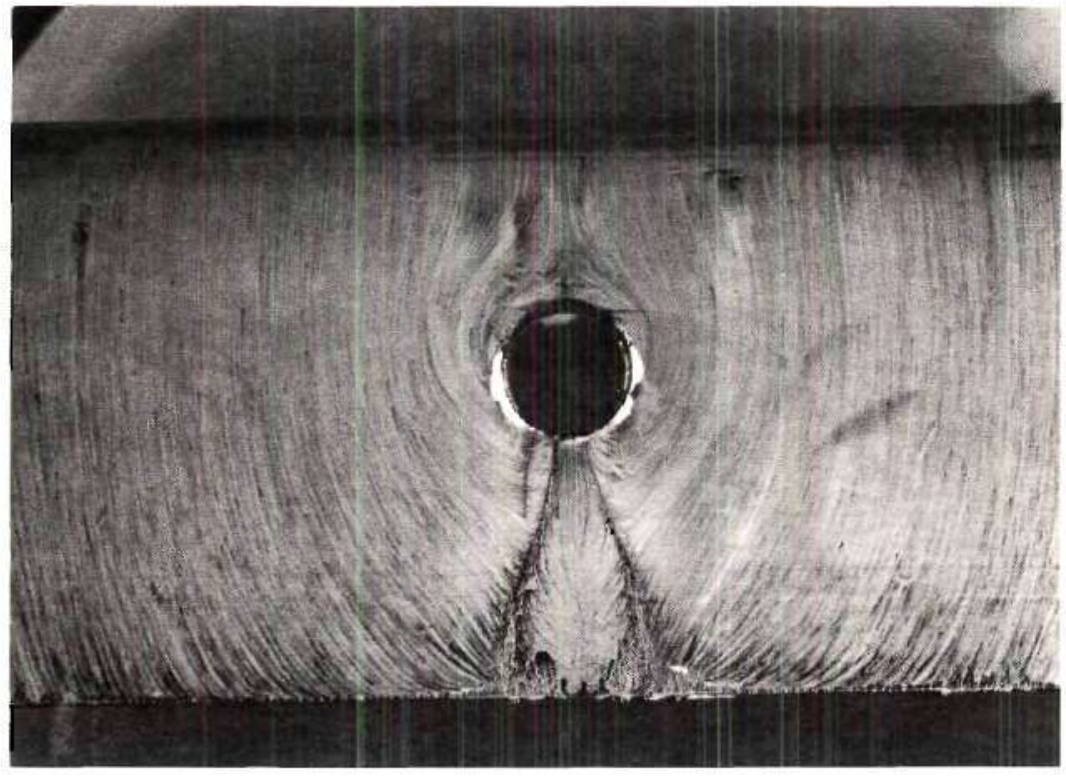

(a) Lower Surface

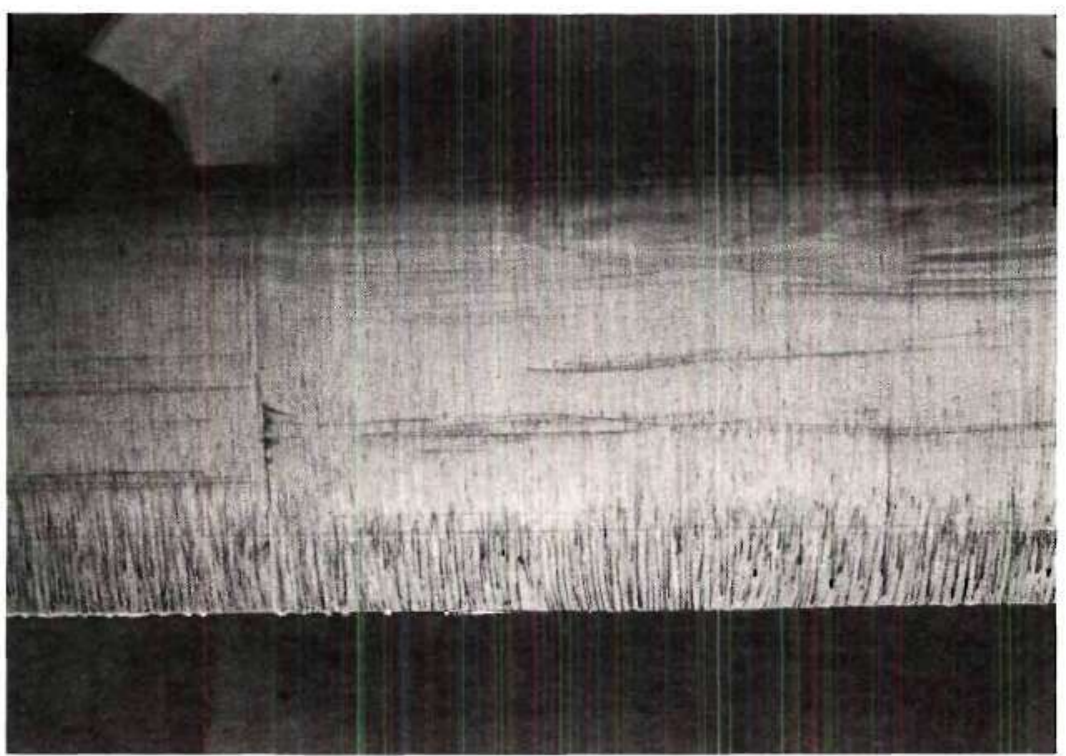

(b) Upper Surface

Figure 36. Oil Flow for the 3. 0 Inch Diameter Jet at

$$
\lambda=4 \text { and } \alpha=0^{\circ} \text {. }
$$




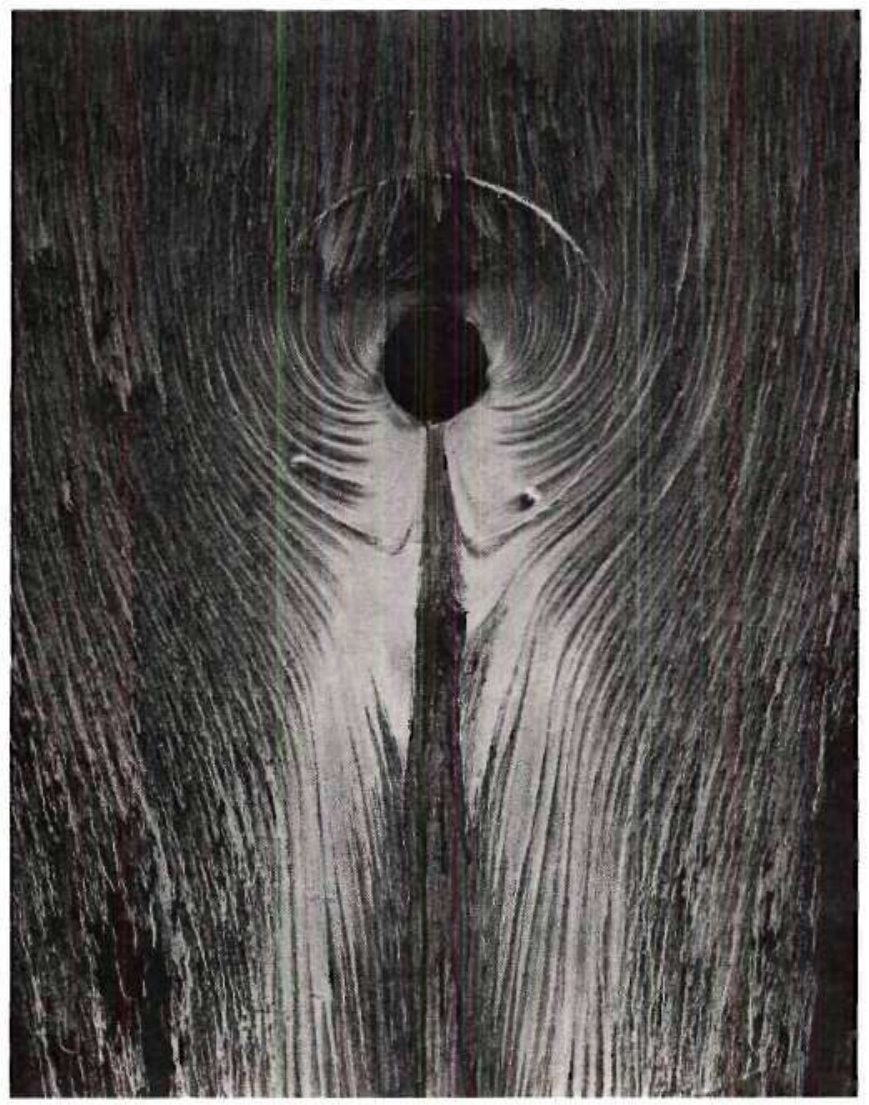

Figure 37. Oil Flow on a Flat Plate with a 2.0 Inch Diameter Jet at $\lambda=4$ (From Reference 15). 


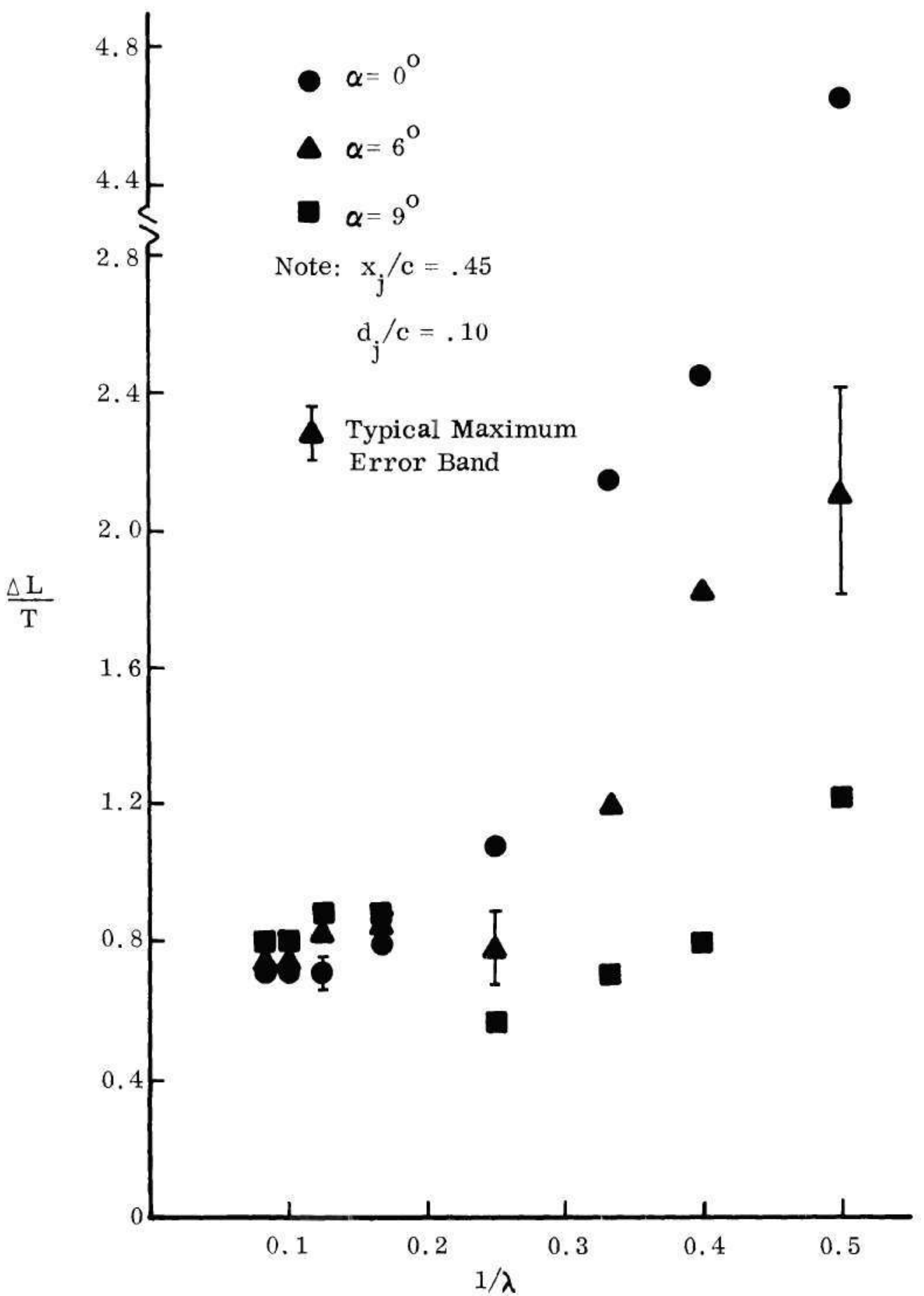

Figure 38. Typical Variation of Interference Lift Coefficient

with Angle of Attack. 


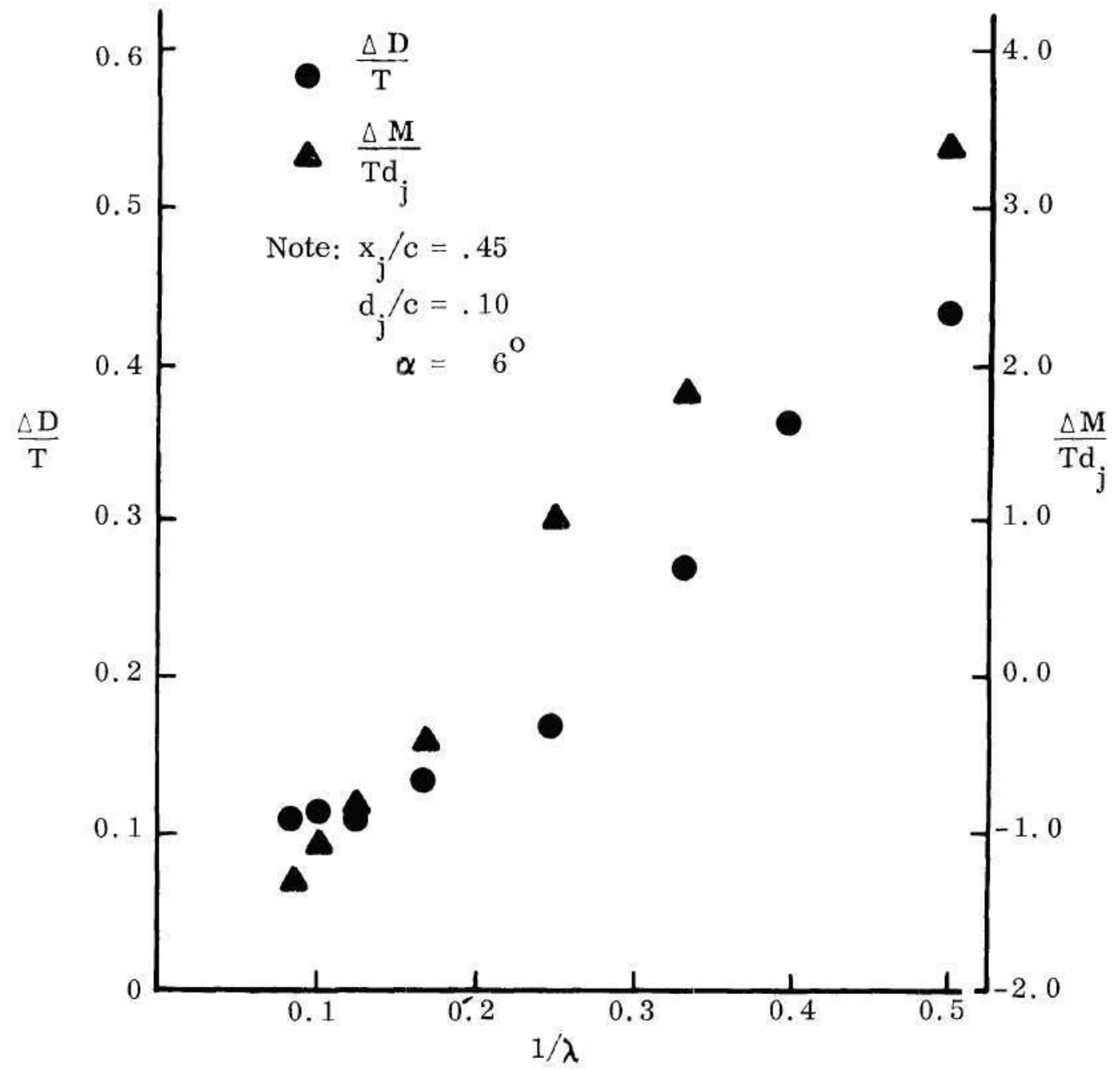

Figure 39. Typical Behavior of the Interference Drag Coefficient and the Interference Pitching Moment Coefficient. 


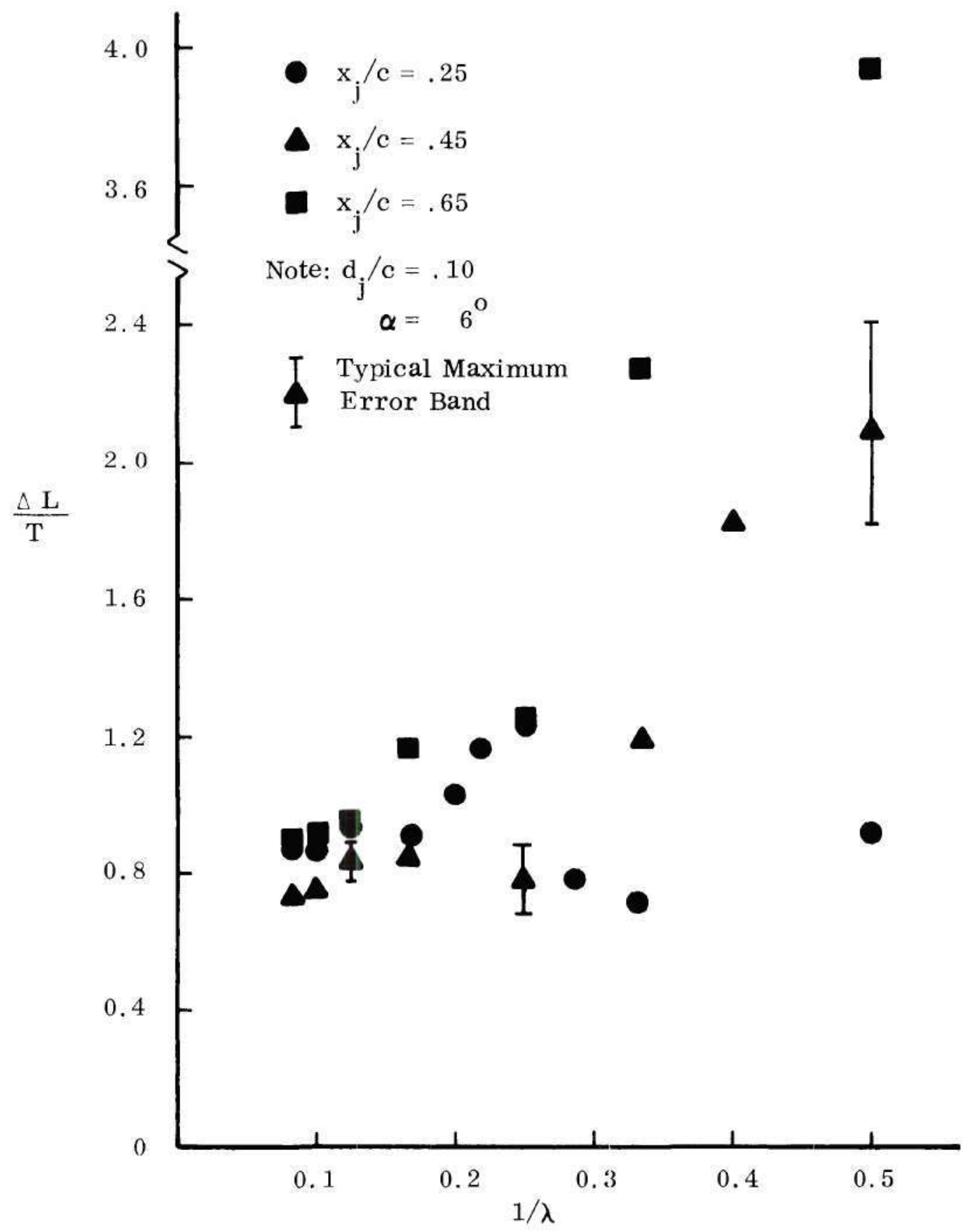

Figure 40. Typical Variation of Interference Lift Coefficient with Jet Exit Location. 


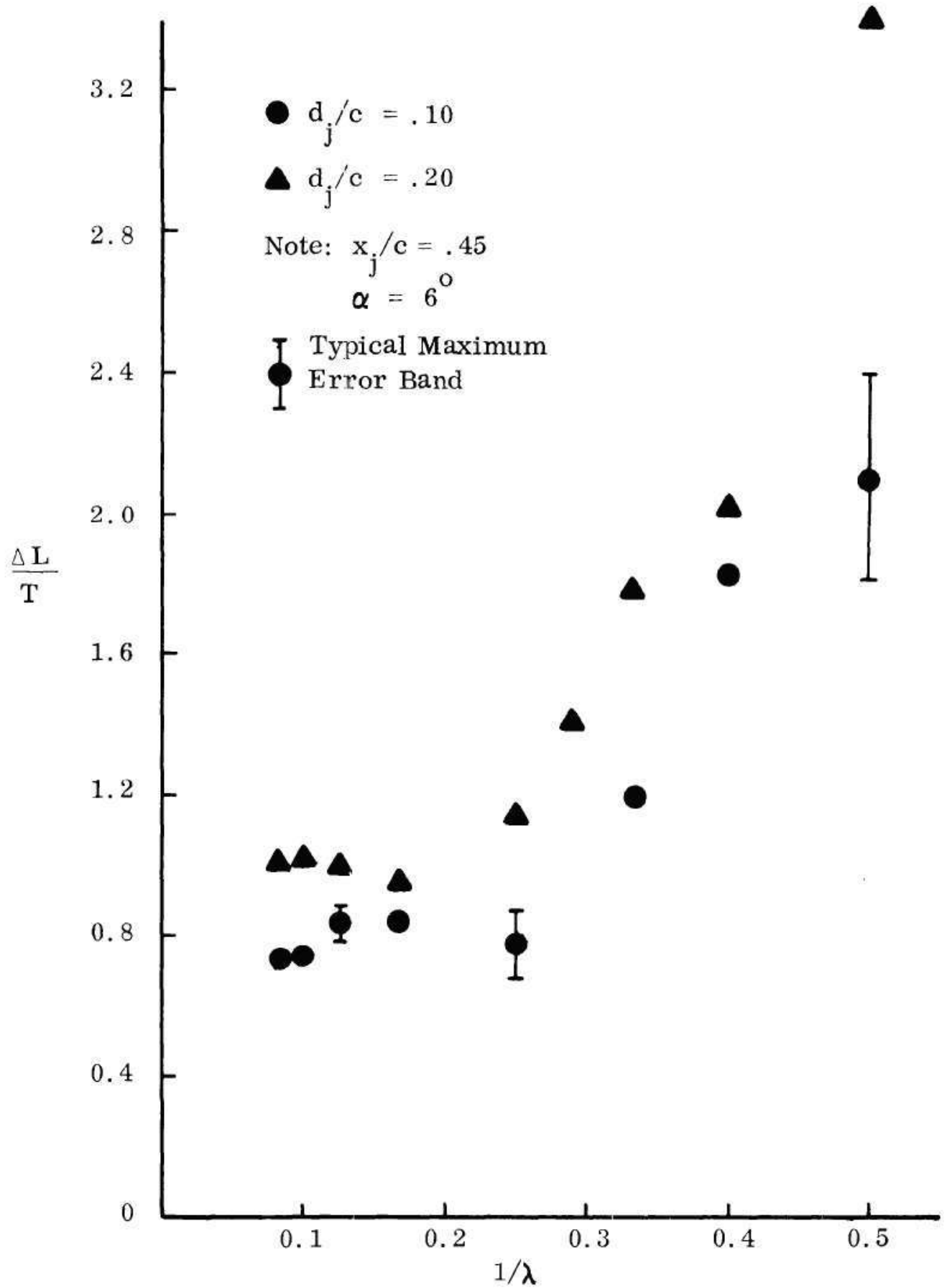

Figure 41. Typical Variation of Interference Lift Coefficient with Jet Exit Size. 


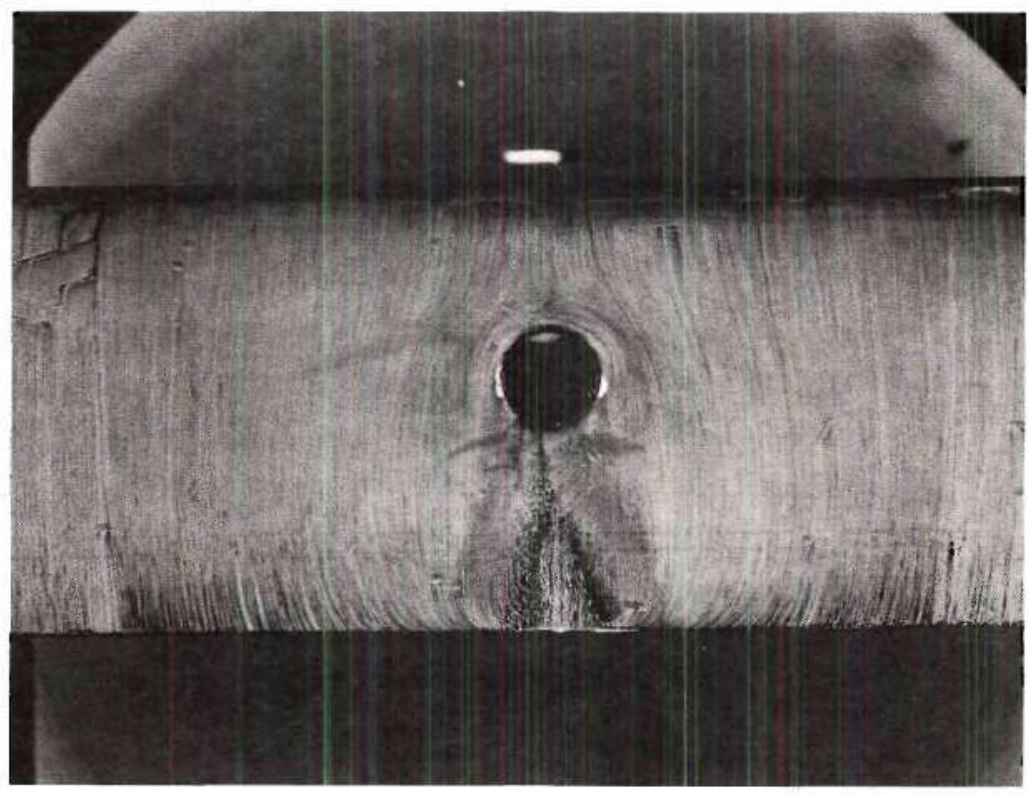

(a) Lower Surface

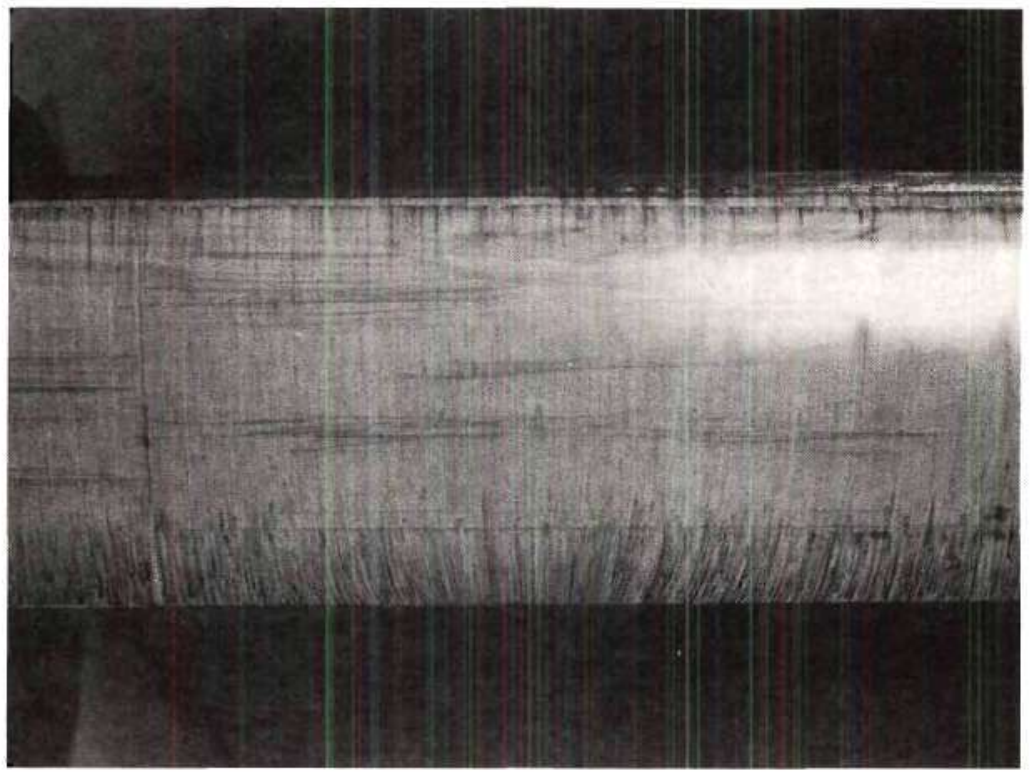

(b) Upper Surface

Figure 42. Oil Flow for the 3.0 Inch Diameter Jet at

$$
\lambda=2 \text { and } \alpha=0^{\circ} .
$$




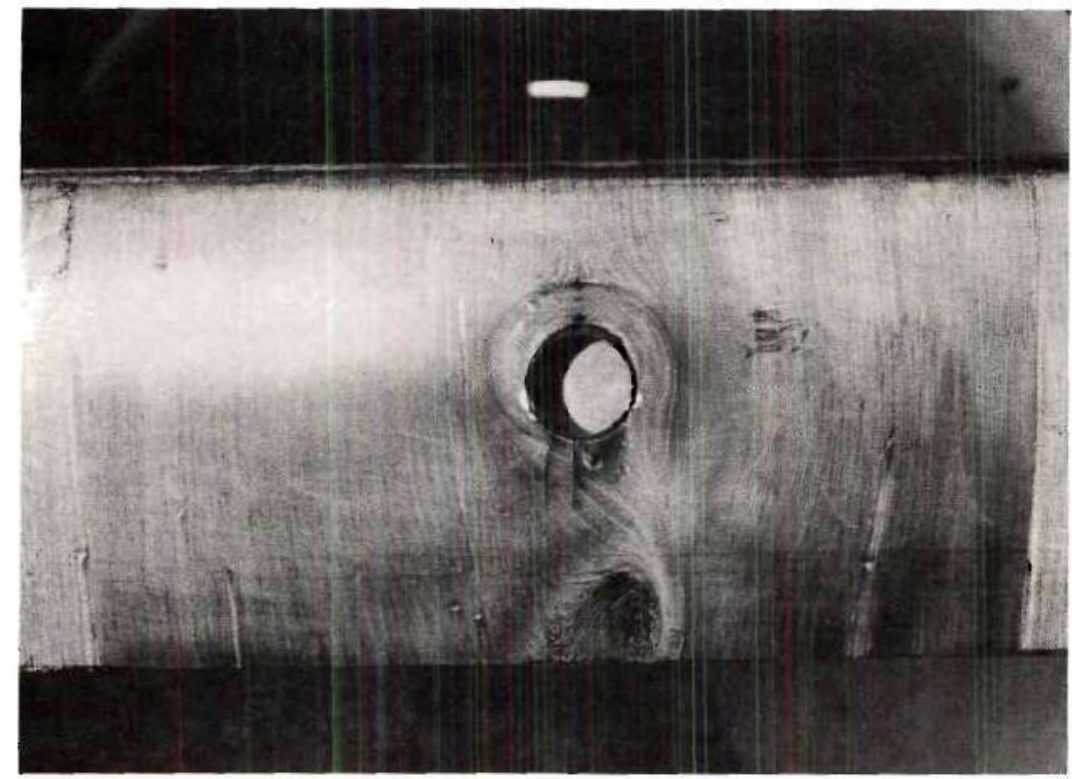

(a) Lower Surface

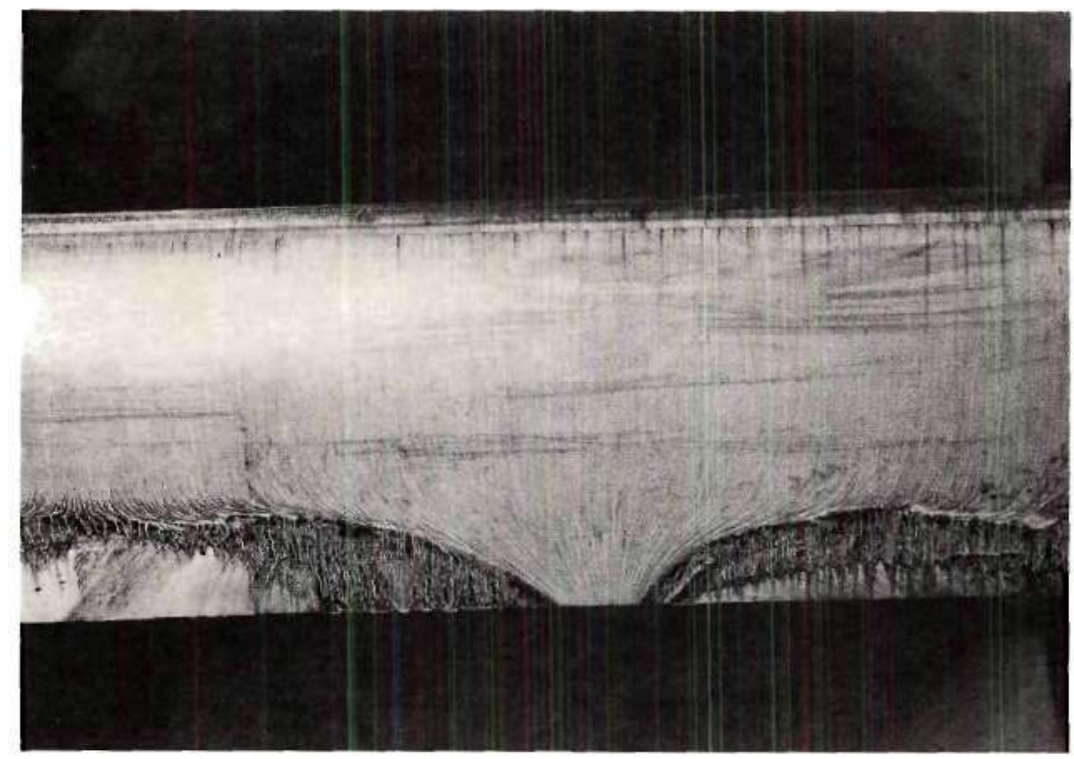

(b) Upper Surface

Figure 43. Oil Flow for the 3.0 Inch Diameter Jet at

$$
\lambda=2 \text { and } \alpha=9^{\circ} \text {. }
$$




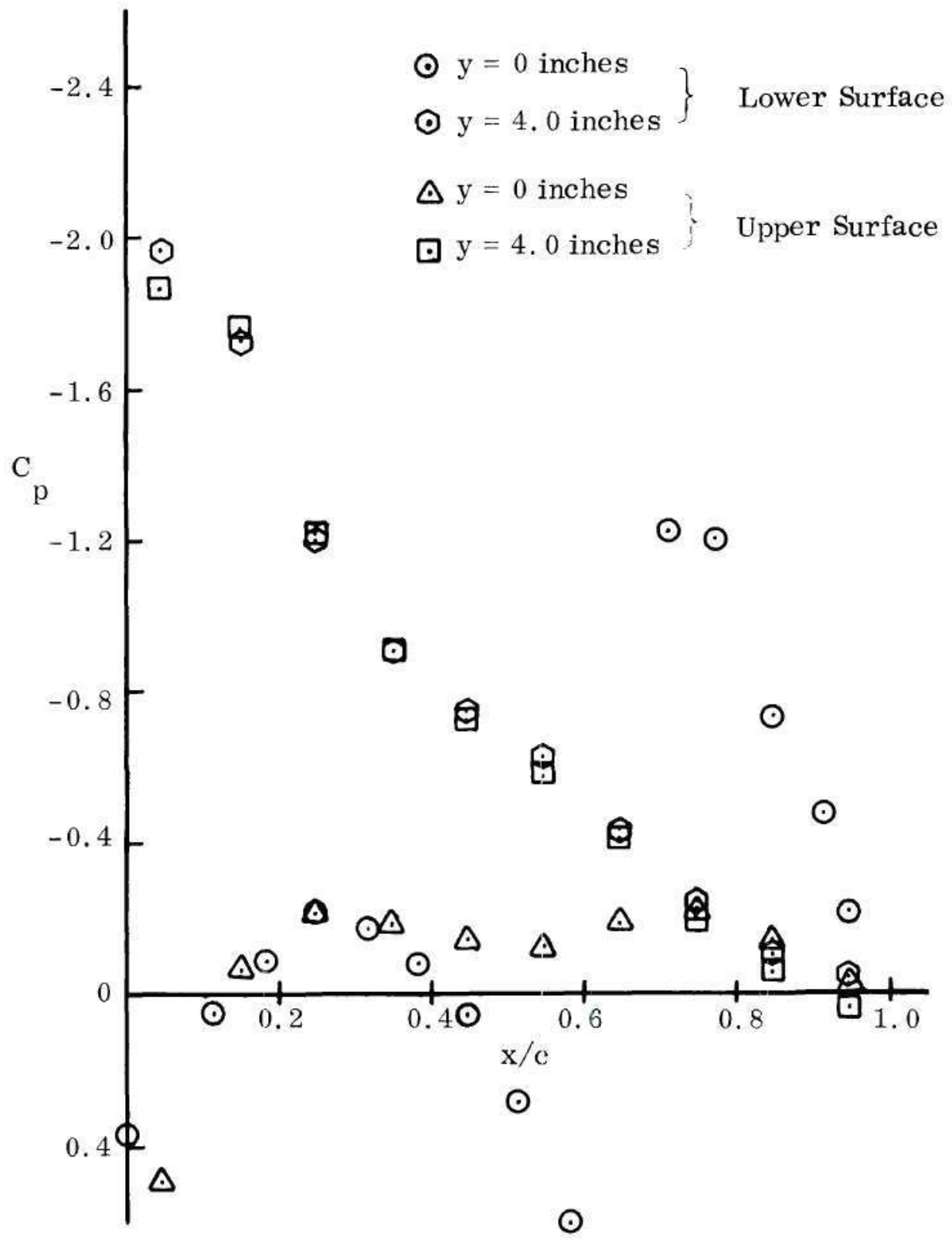

Figure 44. Chordwise Pressure Distributions for $x_{j} / c=0.65, d_{j} / c=0.10$, and $\lambda=2$ at $\alpha=6^{\circ}$. 


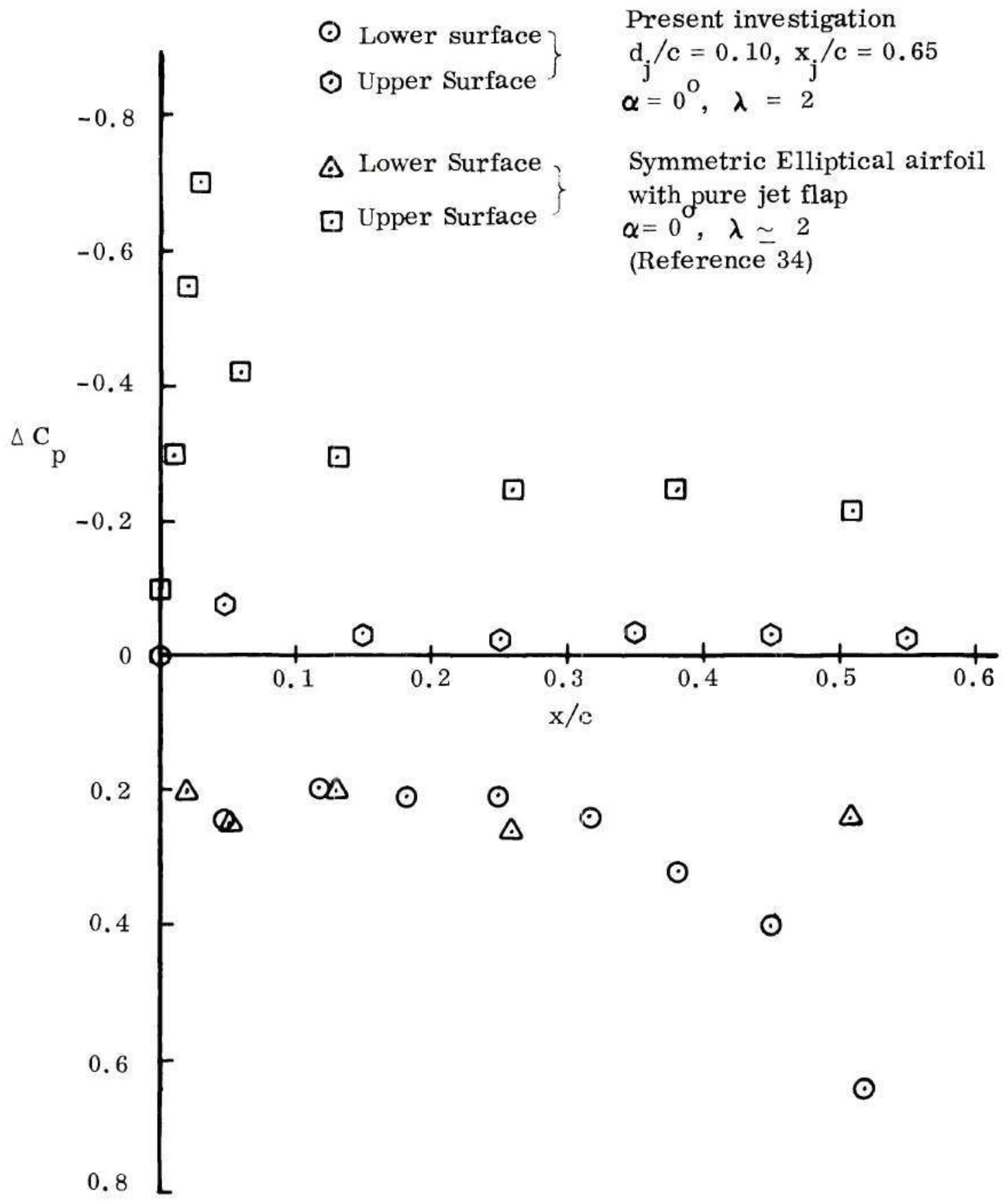

Figure 45. Comparison of the Chordwise Interference Pressure Distribution of the Present Model at the Wing Centerline with that of a Two-Dimensional Symmetric Elliptical Airfoil Equipped with a Pure Jet-Flap (Reference 34). 


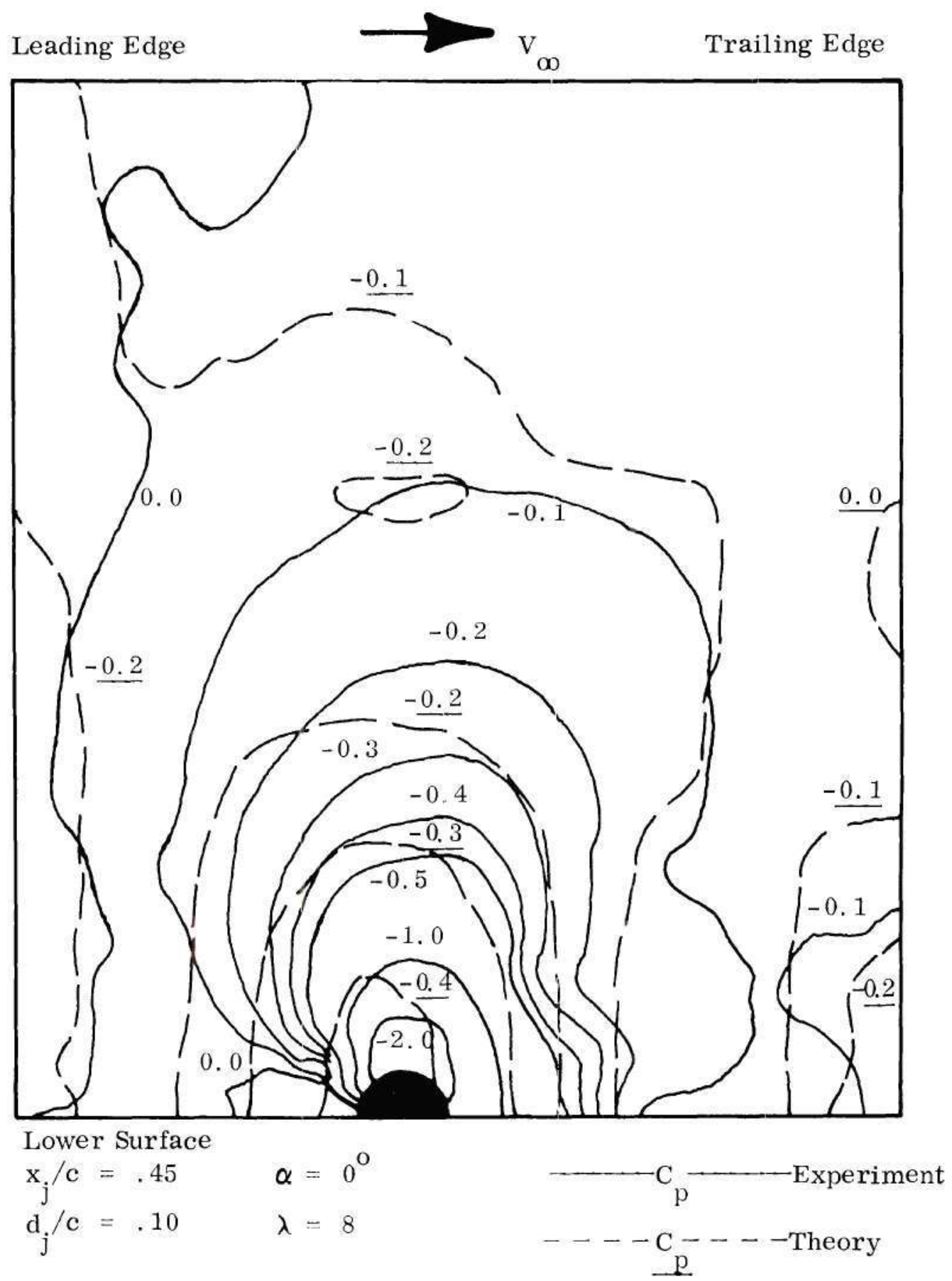

Figure 46a. Comparison of the Wing Interference Surface Pressure Contours with the Theoretical Calculations of Wooler. 


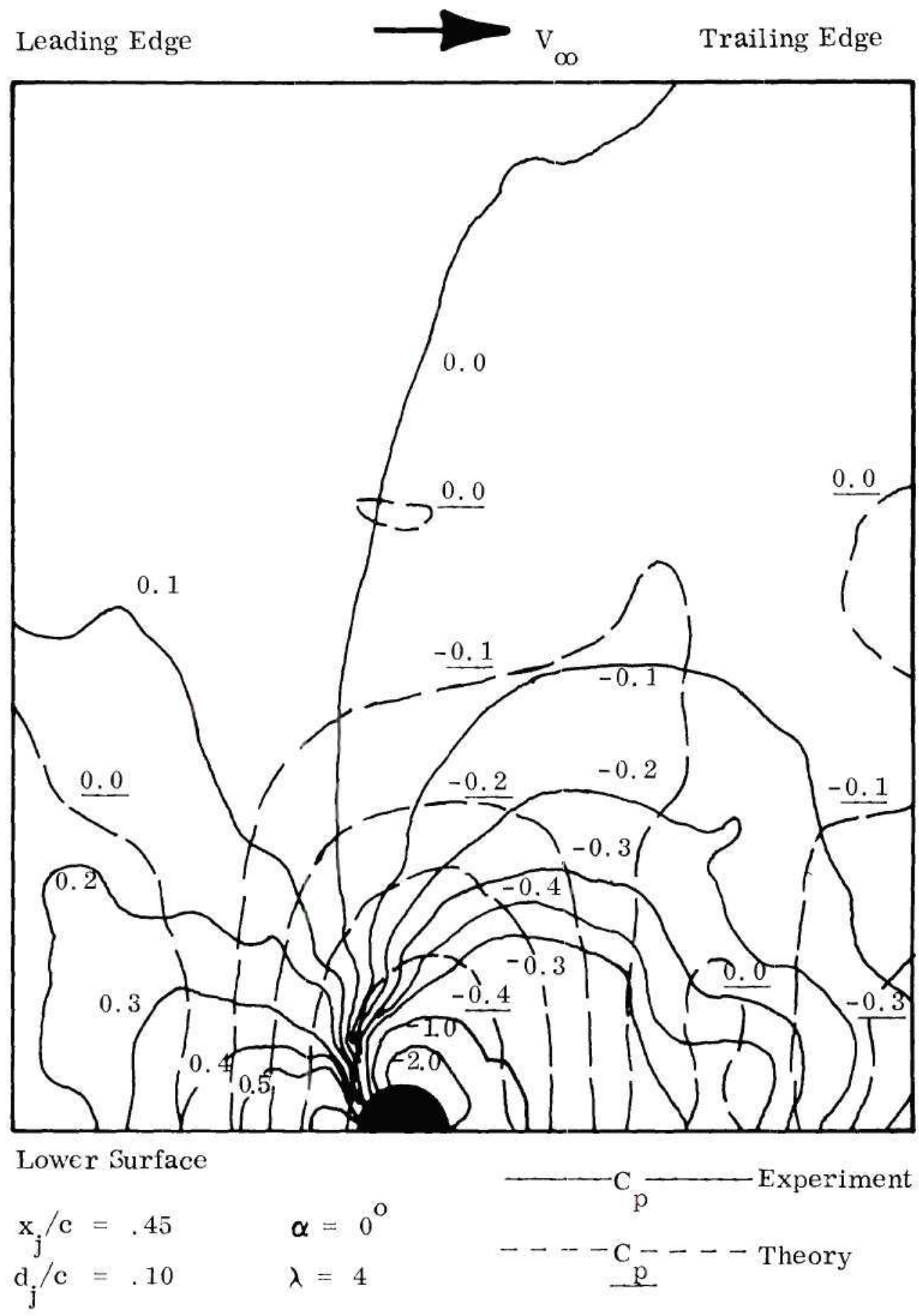

Figure 46b. Comparison of the Wing Interference Surface Pressure Contours with the Theoretical Calculations of Wooler. 
Leading Edge

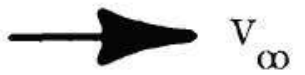

Trailing Edge

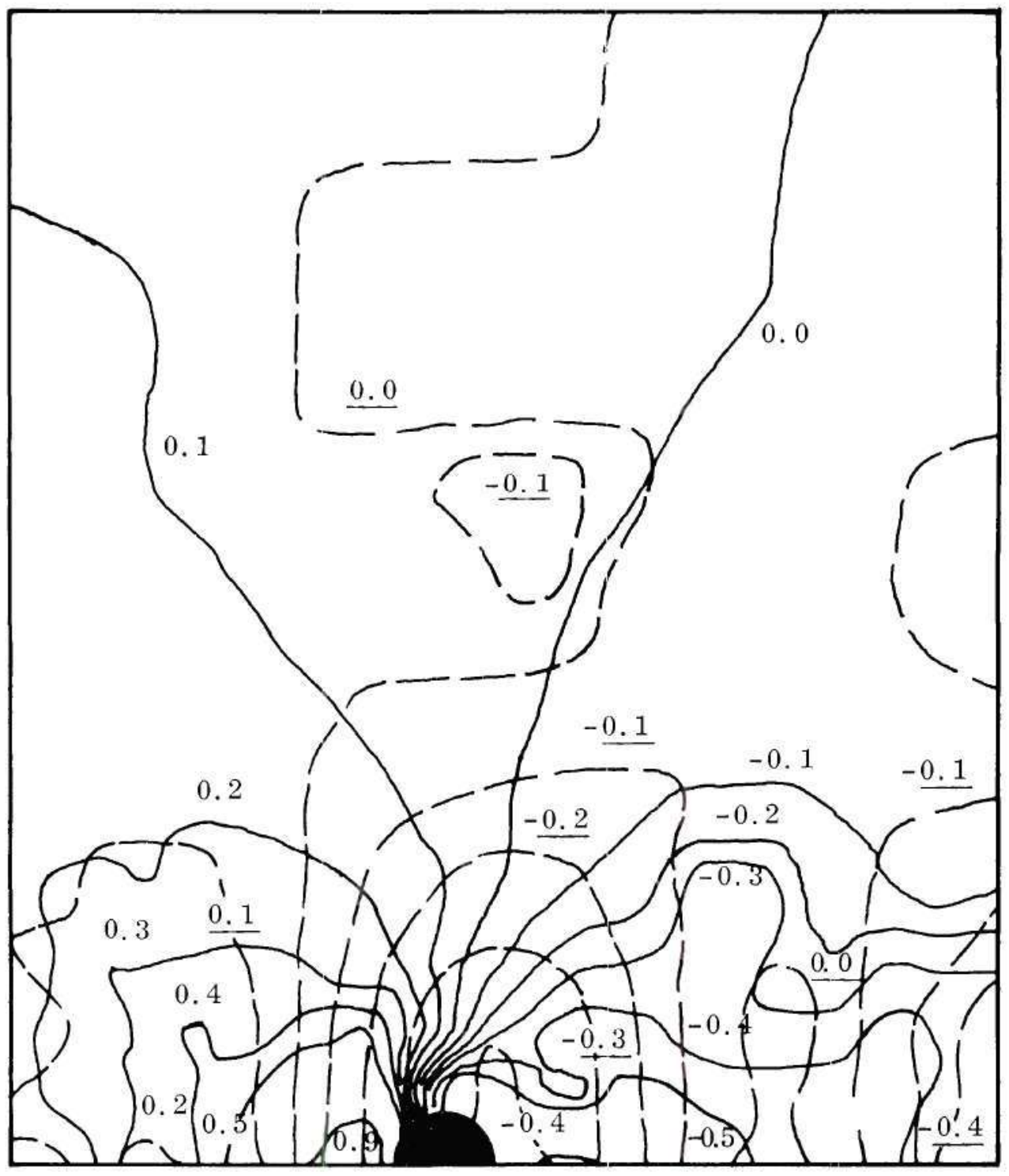

Lower Surface

$$
\begin{array}{ll}
\mathrm{x}_{\mathrm{j}} / \mathrm{c}=.45 & \alpha=0^{\circ} \\
\mathrm{d}_{\mathrm{j}} / \mathrm{c}=.10 & \lambda=2
\end{array}
$$

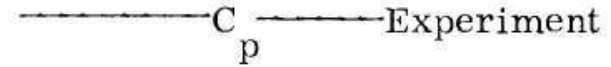

$-\cdots-{ }_{\underline{p}}-\cdots$ Theory

Figure 46c. Comparison of the Wing Interference Surface Pressure

Contours with the Theoretical Calculations of Wooler. 
Leading Edge

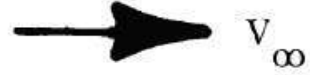

Trailing Edge

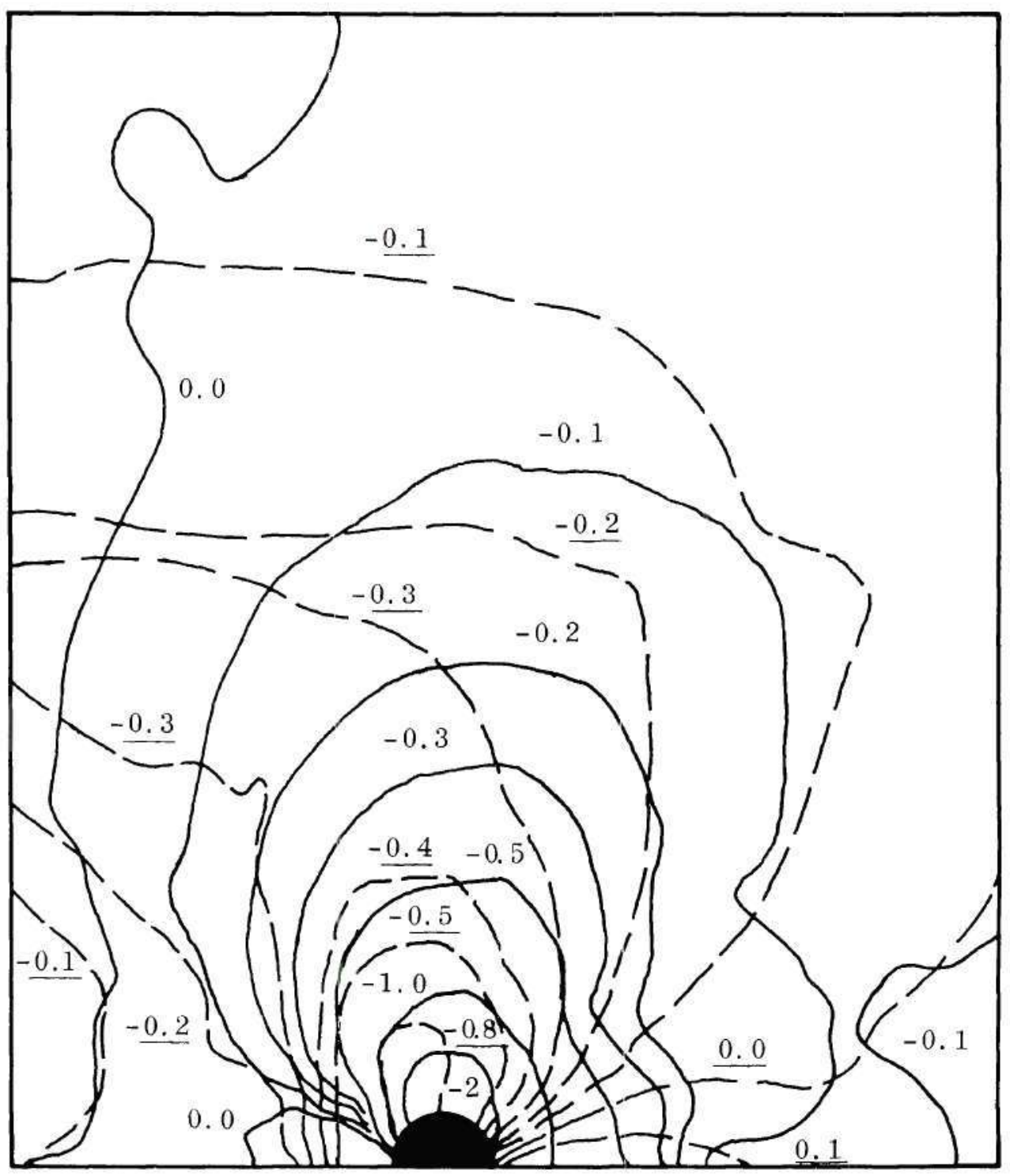

Lower Surface

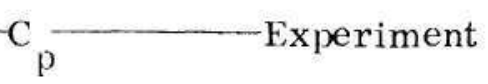

$-\cdots-\mathrm{C}_{\mathrm{p}}-\cdots--$ Theory

$$
\begin{array}{ll}
\mathrm{x}_{\mathrm{j}} / \mathrm{c}=.45 & \boldsymbol{\alpha}=0^{\circ} \\
\mathrm{d}_{\mathrm{j}} / \mathrm{c}=.10 & \lambda=8
\end{array}
$$

Figure 47. Comparison of the Wing Interference Surface Pressure Contours with the Theoretical Calculations of Wasson and Kao. 


\section{APPENDIX A}

\section{CHARACTER OF NOZZLE FLOWS}

Prior to the installation of the plenum chamber in the wing, a test was performed to determine the characteristics of the flow in the nozzles to be used in the experiment. This test consisted of running the nozzles as free jets and making static and total pressure surveys in the jet exit plane as well as determining the jet centerline velocity decay. A measurement of the length and shape of the constant velocity core was also made for one of the jets.

Motivation for this preliminary test was provided by results reported by

Gentry and Margason. ${ }^{36}$ In that work, different nozzles which had varying degrees of uniformity of the jet flow in the exit plane were tested. The results indicated that the aerodynamic interference was strongly dependent on the characteristics of the flow in the nozzles. Hence, for the present investigation, it was desired to insure that all of the jet exit geometries used in the experiment had similarly uniform flow characteristics.

\section{Procedure}

The plenum chamber and air supply hoses were assembled and placed in an area where the jet could exhaust vertically some 20 feet before encountering any obstruction. The air supply system was identical to that eventually installed in the model. 
A modified micromanometer stand served as the traversing mechanism for measurements in the jet exit plane. A total pressure probe and a static pressure probe were interchangeable in the device and could be positioned anywhere in the jet exit plane with an error of less than \pm 0.010 inch. Above the jet exit plane, the micromanometer stand was placed on a mechanical lifter and the vertical height of the probes above the jet exit was set to within $\pm 1 / 16$ inch.

Pressure measurements were made utilizing the Barocel Electronic Manometer System. Pressure lines were connected to two variable capacitance transducers and the output was displayed on a digital voltmeter. One channel was used to monitor plenum chamber total pressure while the other was used for the pressure probe measurement. In both cases, the reference side of the transducer was exposed to ambient pressure. Plenum chamber stagnation temperature was monitored using the chromel-alumel thermocouple installed in the chamber.

Because the jet air is heated by compression in the centrifugal blower supplying the air, the test technique employed was to set the desired plenum total pressure after the plenum stagnation temperature had risen to an equilibrium value. For the 1.5 inch jets this was $130^{\circ} \mathrm{F}-140^{\circ} \mathrm{F}$; and for the 3.0 inch jet about $160^{\circ} \mathrm{F}$. The pressure survey in the exit plane was then made from one edge of the jet to the other.

In each case, at least two different nozzle radials were traversed. (Usually these were $\beta=45^{\circ}$ and $\beta=-45^{\circ}$ or $0^{\circ}$.) Ambient conditions and plenum total pressure were recorded before and after each traverse and any changes were accounted for in the data reduction by averaging. 


\section{Jet Exit Velocity Profiles}

The free jet data was reduced by using the measured total pressure, $p_{o j}$, and static pressure, $\mathrm{p}_{\mathbf{s j}}$, to compute local jet velocities. This was done by first computing the local Mach number given by

$$
M_{j}=\left\{\frac{2}{\gamma-1}\left[\left(\frac{p_{o j}}{p_{s j}}\right)^{\frac{\gamma-1}{\gamma}}-1\right]\right\}^{\frac{1}{2}},
$$

then computing the local speed of sound

$$
c_{j}^{\prime}=\left[\gamma \bar{R} \frac{\bar{T}_{o j}}{\left(1+\frac{\gamma-1}{2} M_{j}^{2}\right)}\right]^{-\frac{1}{2}}
$$

and then finally the local velocity

$$
V_{j}=c_{j}^{\prime} M_{j}
$$

These local velocities were non-dimensionalized by computing an isentropic jet exit velocity, $\mathrm{V}_{\text {jo }}$, based on the plenum total pressure and assuming that the jet static pressure at the exit was equal to the ambient pressure.

Figures A-1 and A-2 summarize the exit velocity profiles of the jet geometries which were tested in the wing. The profiles were judged to be satisfactorily uniform. Note, however, that in some cases (Figure A-2b, for example) the average local jet velocity at the exit plane is significantly different from the velocity computed based on the plenum chamber total pressure. This discrepancy, which 
arises because the flow in the plenum chamber/nozzle is non-isentropic, was taken into account during the actual crossflow testing where the jet exit velocity was set by a suitable correction to the plenum chamber stagnation pressure.

Several of the jet exit geometries displayed unacceptable velocity profiles when first tested and modifications internal to the plenum chamber were required. The velocity profiles of the 1.5 inch jet at the most aft exit location are shown in Figure A-3 before and after modification. The poor original profile was attributed to the step in the plenum chamber just forward of the bellmouth at this location. A satisfactory profile was obtained by installing a false bottom in the plenum chamber such that the step no longer existed.

The larger 3.0 inch jets presented a more difficult problem primarily because of the limited clearance height available to the bellmouth. After considerable experimentation with a 3.0 inch jet exiting at the 25 percent chord location (not used in this series of tests), the combination of a cylindrical fine mesh screen extending from the bellmouth to the opposite side of the plenum chamber and a baffle the width of the bellmouth and located just aft of it (extending again to the opposite side) yielded results which were deemed satisfactory. A comparison between the original and improved velocity profiles is shown in Figure A-4. In light of these results, a cylindrical screen was also installed on the 3.0 inch jet at the 45 percent chord exit location. The velocity profile in this case (Figure A-2b) was excellent.

Quality of the Free Jet

To further delineate the quality of the free jets, decay characteristics were 
measured. The variation of jet dynamic pressure with distance normal to the jet exit plane is shown in Figures A-5 and A-6. The jet centerline dynamic pressure, $\mathrm{q}_{\mathrm{z}}$, was non-dimensionalized by the jet exit dynamic pressure.

The earlier decay characteristics of the 1.5 inch jet at the 65 percent chord exit and the 3.0 inch jet are most certainly due to the limited clearance between the jet nozzles and the plenum chamber surface. However, the decay was considered acceptable since the data indicated that similar turbulence levels existed for all jets in the exit plane. This resulted in the jet exit plane velocity being maintained for at least a few diameters from the exit.

Finally, a detailed survey was made to determine the length and shape of the constant velocity core for the $1.5 \mathrm{inch}$ jet at the 45 percent chord exit location. This locus of velocities equal to the jet exit velocity is shown in Figure A-7. The traverses were made on the $0^{\circ}$ radial and the $45^{\circ}$ radial of the jet with virtually identical results (see Figure A-8 for example) and hence this shape can be considered to be symmetrical.

In summary, the characteristics of the jet exit flows are quite similar for all exit geometries and thus comparisons of the resulting aerodynamic interference under crossflow conditions between the respective geometries should be meaningful. 

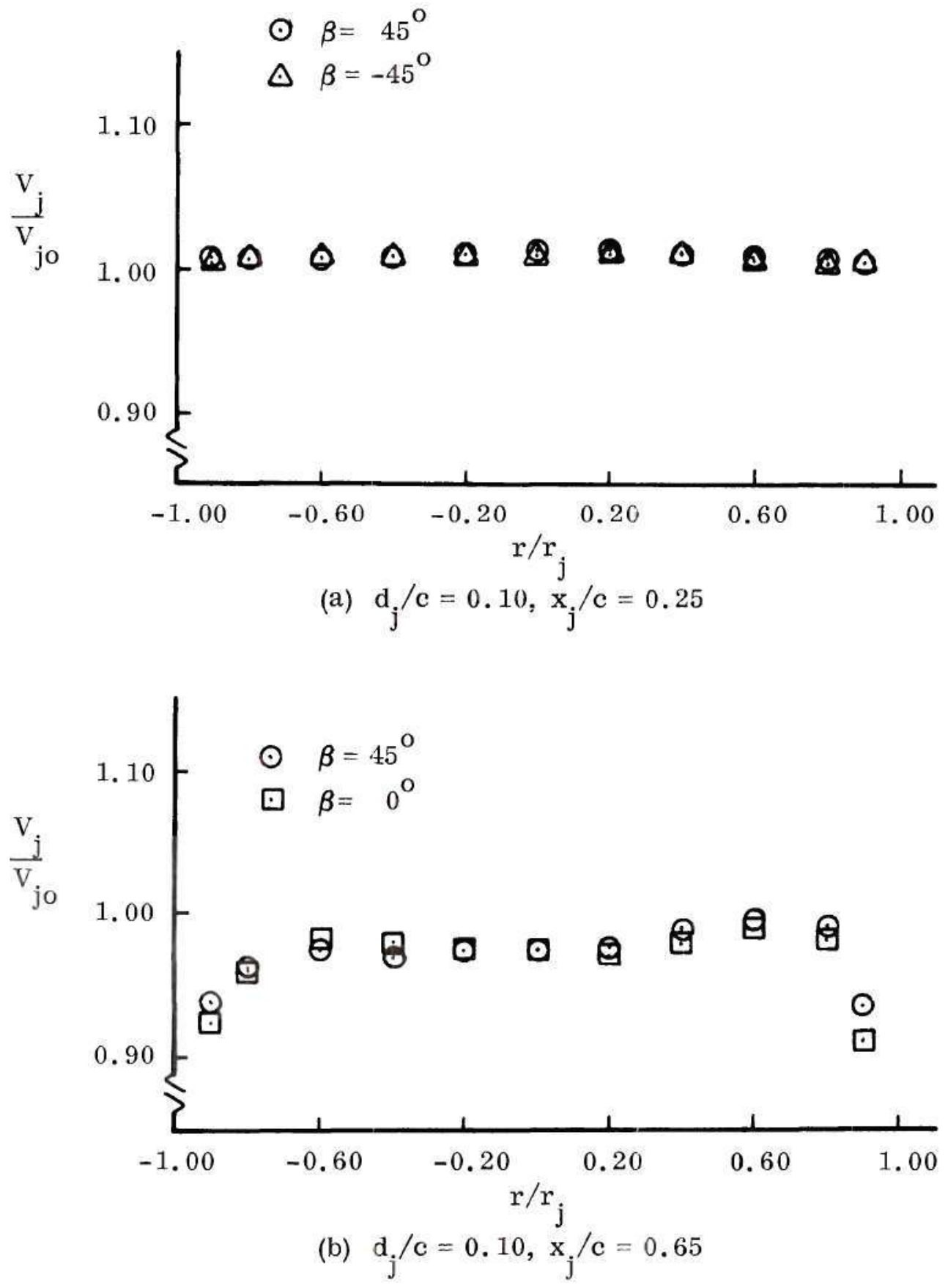

Figure A-1. Free Jet Exit Plane Velocity Profiles for the 1.5 Inch Jet at the 25 and 65 Percent Chord Exit Locations. 


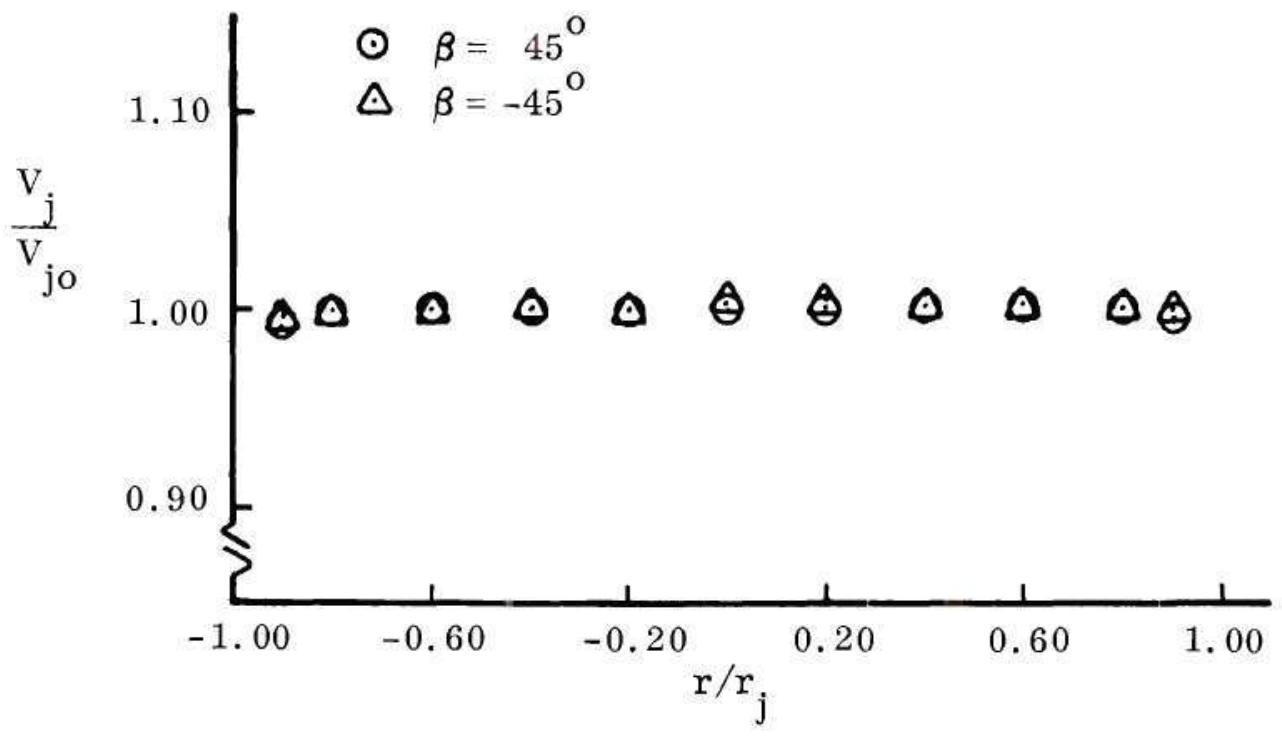

(a) $\mathrm{d}_{\mathrm{j}} / \mathrm{c}=0.10, \mathrm{x}_{\mathrm{j}} / \mathrm{c}=0.45$

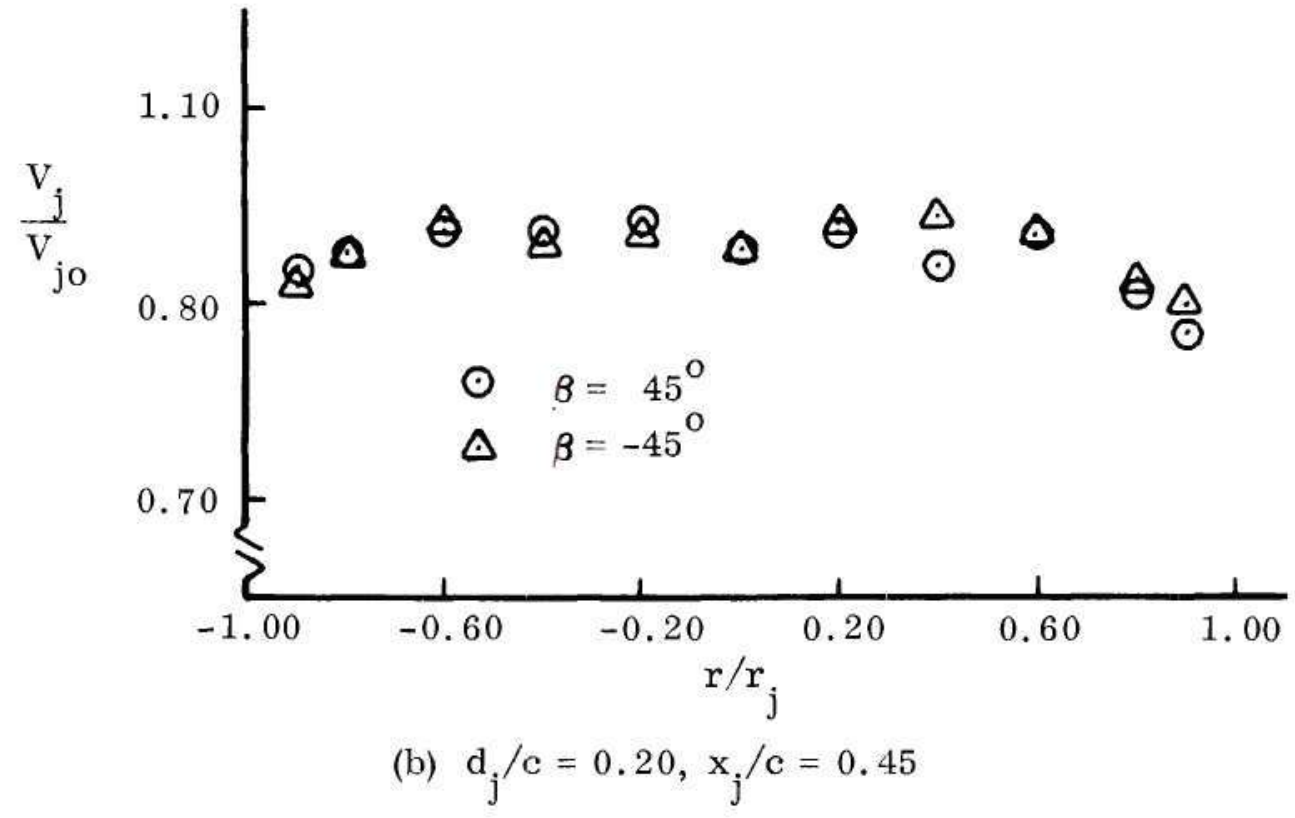

Figure A-2. Free Jet Exit Plane Velocity Profiles for the 1.5 Inch Jet and the 3.0 Inch Jet at the 45 Percent Chord Exit Location. 


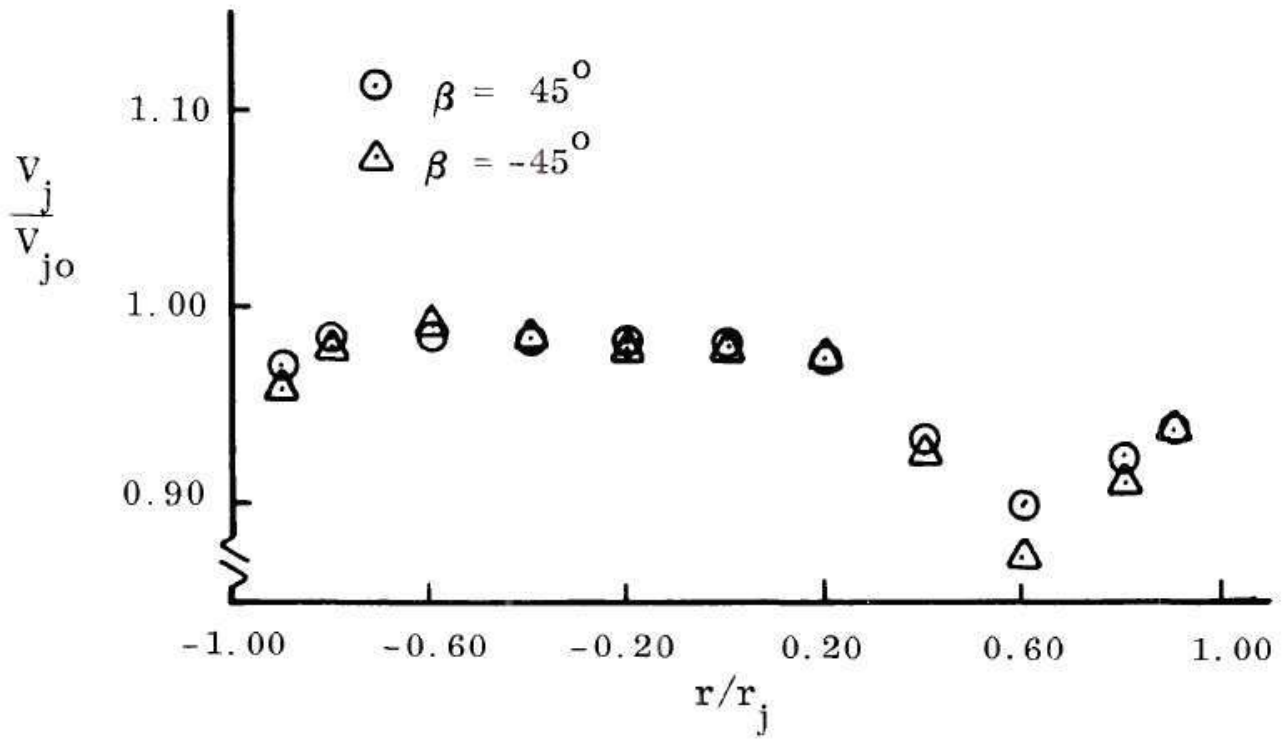

(a) Before Modification

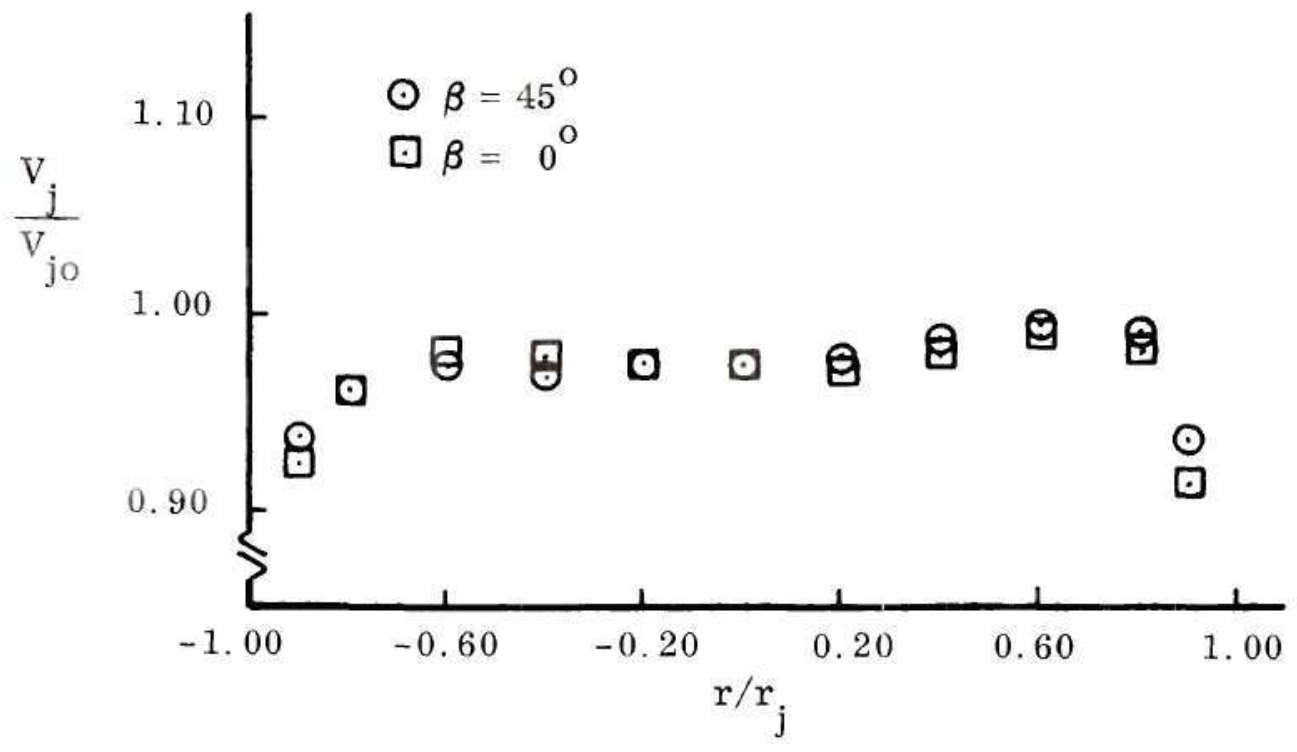

(b) After Modification

Figure A-3. Free Jet Exit Plane Velocity Profiles for the 1.5 Inch Jet at the 65 Percent Chord Exit Location Before and After Modification. 


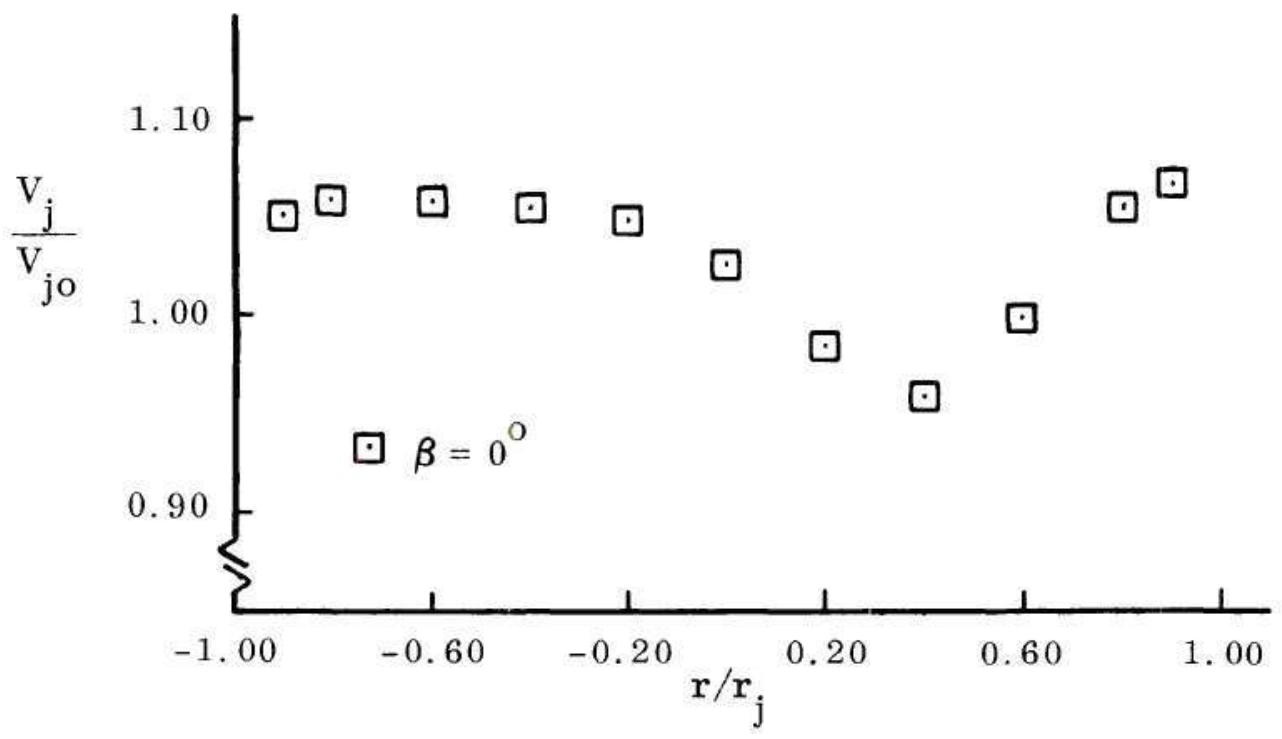

(a) Before Modification

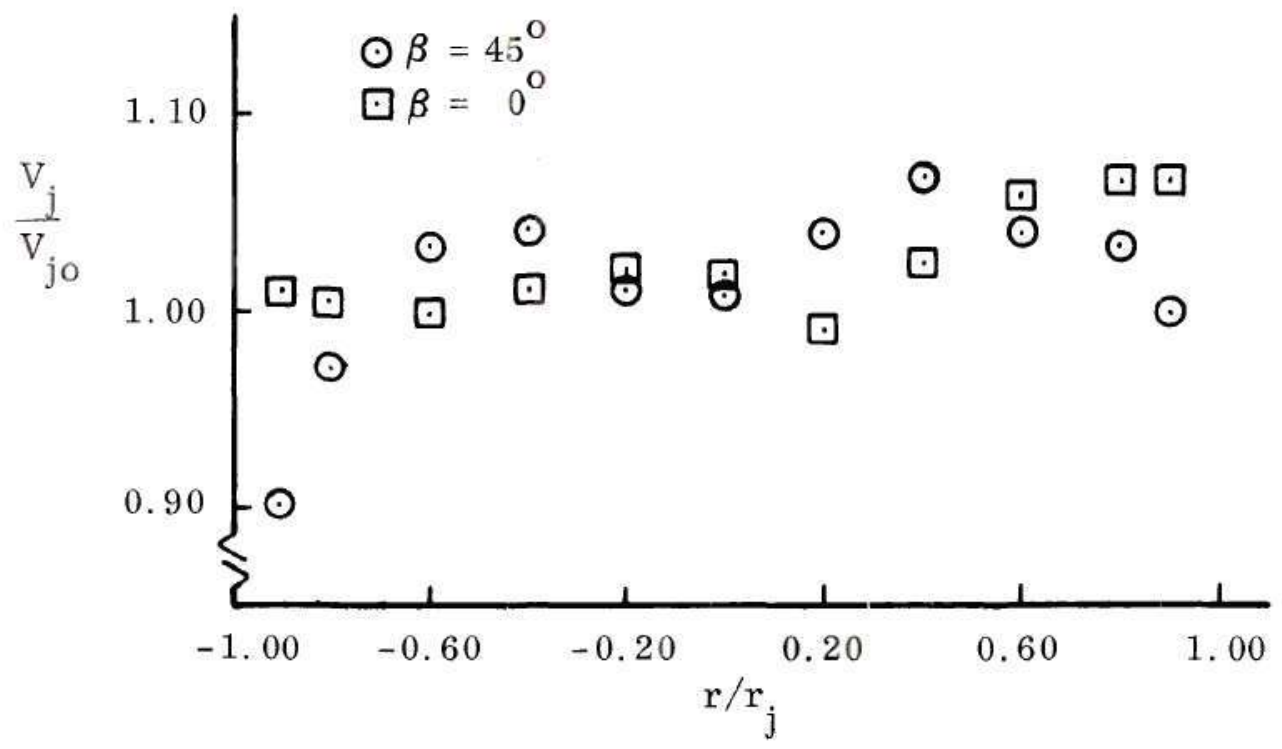

(b) After Modification

Figure A-4. Free Jet Exit Plane Velocity Profiles for the 3.0 Inch Jet at the 25 Percent Chord Exit Location Before and After Modification. 


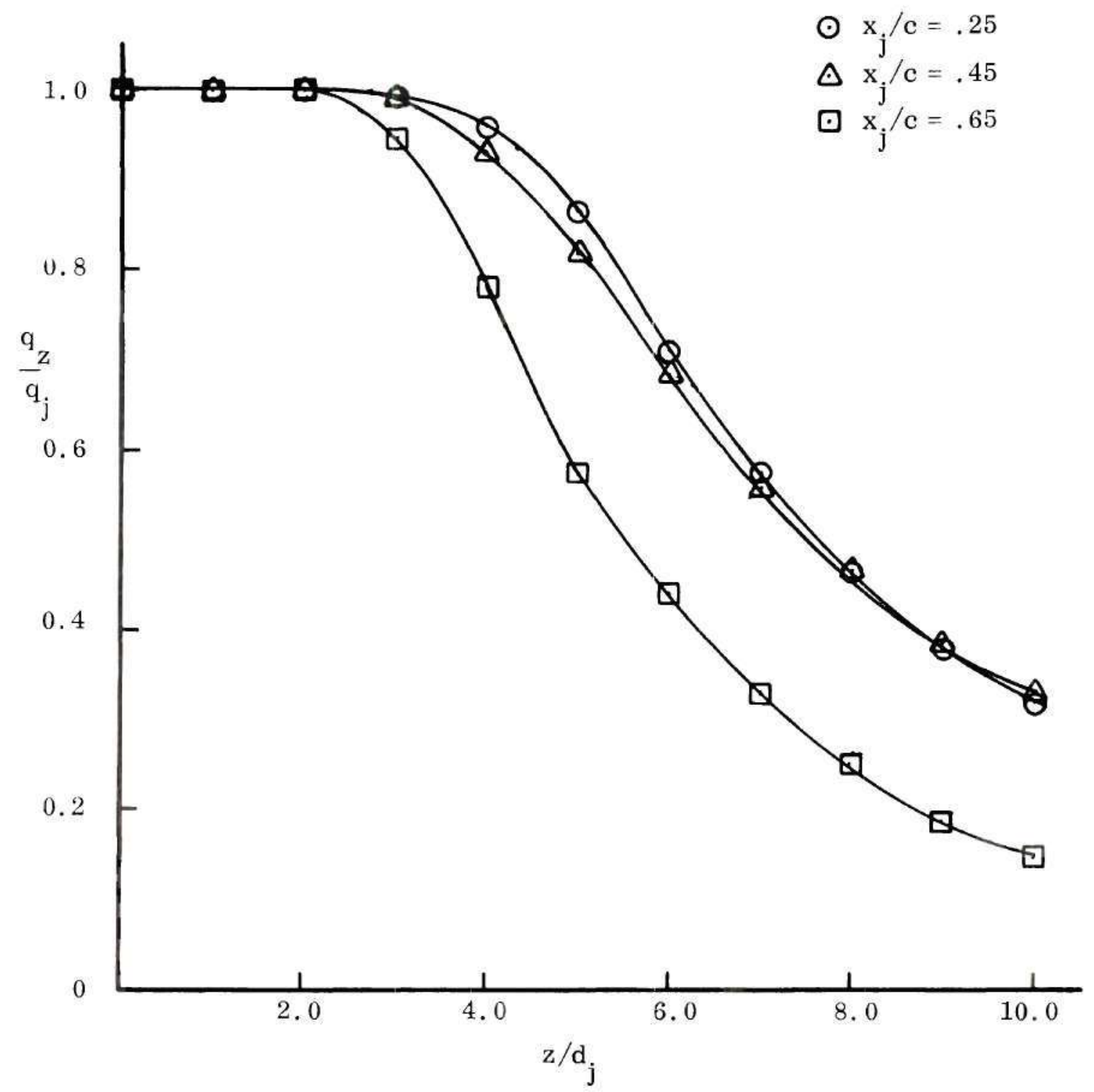

Figure A-5. Free Jet Decay Characteristics for the 1.5 Inch Jets. 


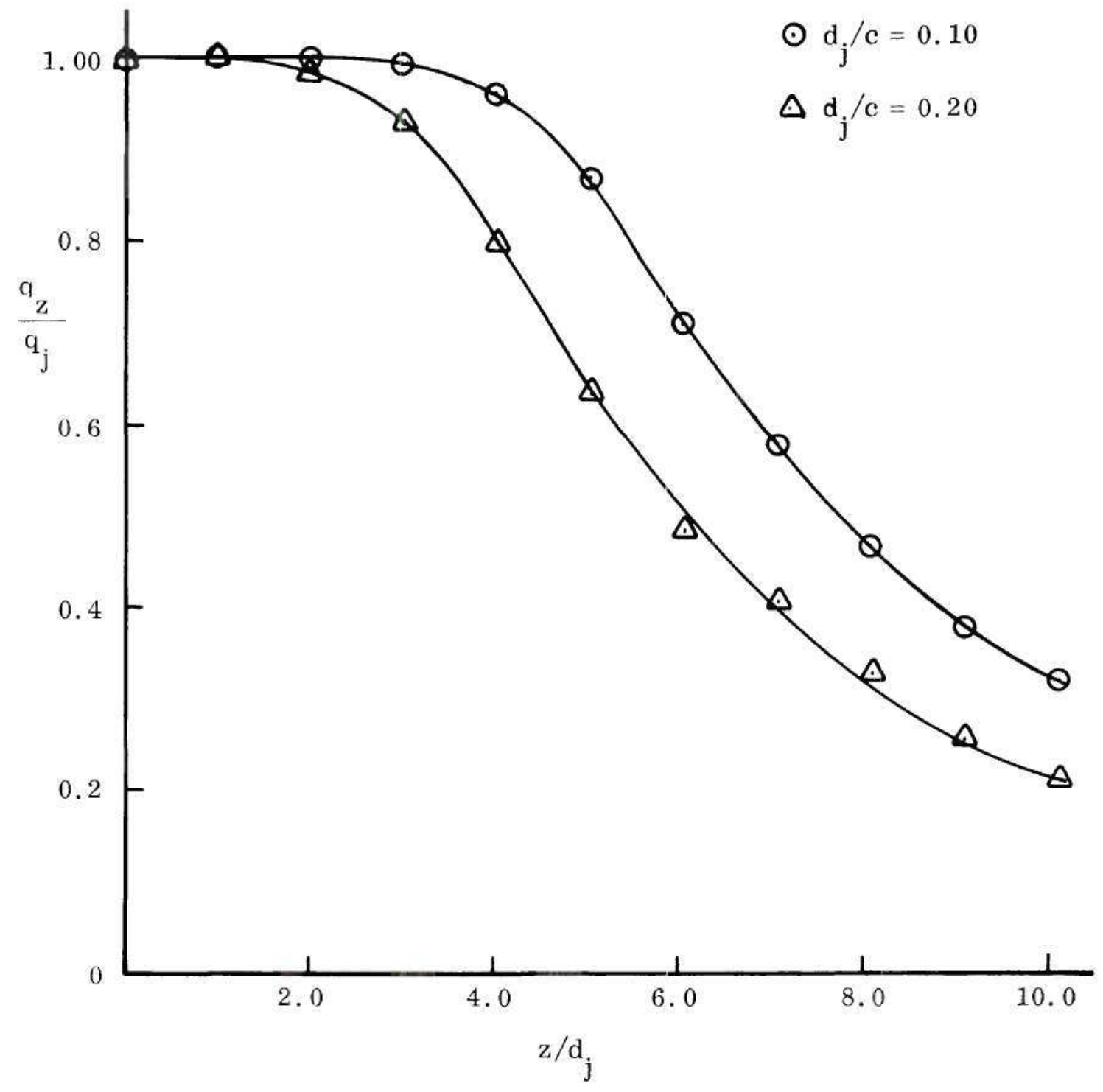

Figure A-6. Free Jet Decay Characteristics for the Jets at the 45 Percent Chord Exit Location. 


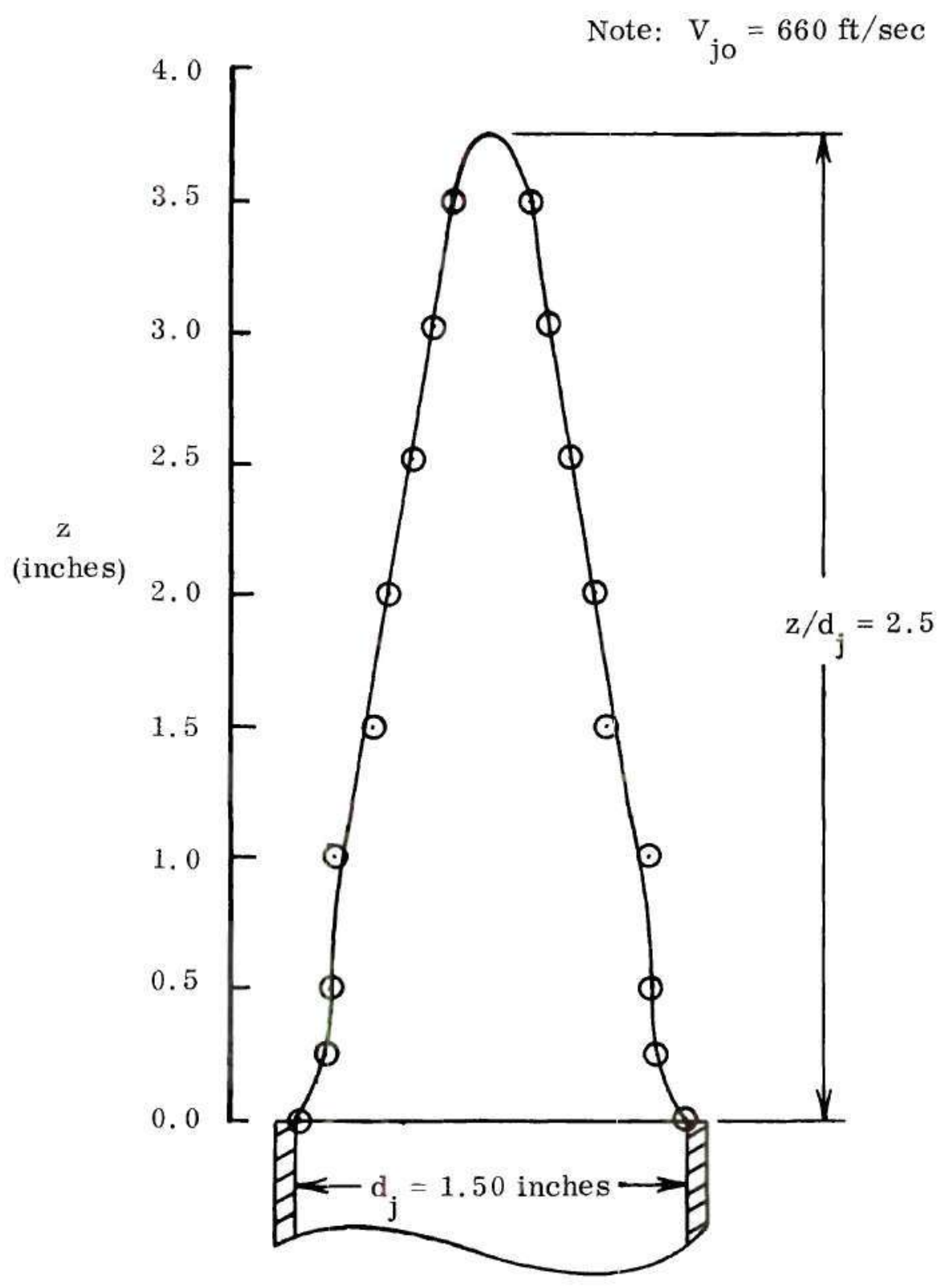

Figure A-7. Free Jet Constant Velocity Core for the 1.5 Inch Jet at the 45 Percent Chord Exit Location. 


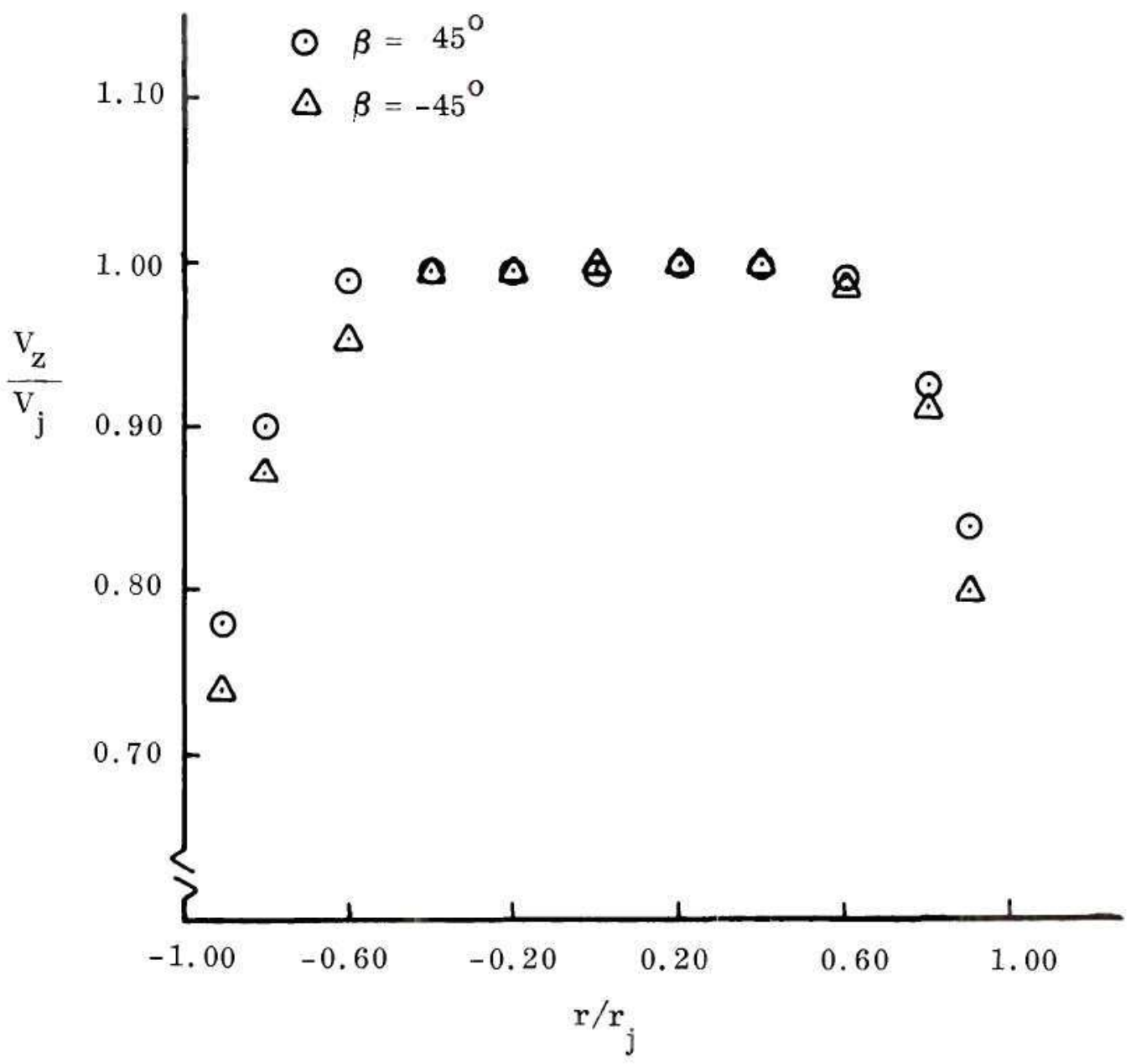

Figure A-8. Free Jet Velocity Profiles for the 1.5 Inch Jet at the 45 Percent Chord Exit Location 2.5 Inches From the Jet Exit Plane. 


\section{APPENDIX B}

\section{CALIBRATION OF TEST INSTRUMENTATION AND TEST FACILITY}

The calibration techniques used to check out and to determine the characteristics of the instrumentation used in the wind tunnel tests are described in this appendix. Results are presented for the wind tunnel balance system, the internal balance system, and the pressure measurement system. The calibration of the wind tunnel freestream after modification of the test section is also included.

\section{Wind Tunnel Balance System}

A calibration of the tunnel balance system was necessary because the model was mounted ten inches above the balance centerline and also to determine the effectiveness of the balance bridge arrangement which was described in Chapter II. Two such calibrations were made--one prior to the start of testing and another when the program was approximately two-thirds completed. The second calibration was motivated by a desire to strengthen the validity of the unexpected interference lift results obtained during the test.

The calibration procedure consisted of a static loading of the model in lift, drag, and then pitch. The balance readings were recorded utilizing the automatic eight channel printer. To facilitate the static loading, a bar was constructed to fit on the wing contour. This bar enabled lift to be loaded on the wing trunnion axis (balance centerline) and drag in the plane of the wing chordline. A pitching moment was applied by loading in the lift direction at a point one foot aft of the tunnion axis. 
The system was loaded in the lift direction to 250 pounds, to 50 pounds in drag, and to 50 foot-pounds in pitch. The individual weights used were accurate to within \pm .01 pounds and no more than five separate weights were required for any loading. Balance readings were taken during both the loading and the unloading of the model to check for any hysteresis.

The results for both lift calibrations are plotted in Figure B-1. The straight lines shown in all figures are determined by a least squares calculation. Figures B-2 and B-3 give the results of the drag and pitching moment calibrations respectively. Since the model was mounted above the balance centerline, there was a significant interaction of drag into pitching moment as shown in figure B-2. The slopes obtained from the least squares straight line fit thus lead to the following equations for the calibrated forces and moment which were used in the data reduction:

$$
\begin{aligned}
\mathrm{L}_{\mathrm{C}} & =.997 \mathrm{~L}_{\mathrm{B}} \\
\mathrm{D}_{\mathrm{C}} & =.998 \mathrm{D}_{\mathrm{B}} \\
\mathrm{PM}_{\mathrm{C}} & =1.008 \mathrm{PM}_{\mathrm{B}} \cdot .823 \mathrm{D}_{\mathrm{C}}
\end{aligned}
$$

where the subscript B indicate the balance readout. These equations are for the second balance calibration. The balance readings indicated no interactions other than that mentioned.

An examination of Figure B-1 indicates only a small difference between the two calibrations. However, by presenting the balance reading as a deviation from 
the applied load as shown in Figure B-4, the difference between the two calibrations becomes distinct. The initial phase of the second calibration consisted of systematically removing components of the installed model which might foul the balance system and making a lift calibration at each stage until finally a free balance was attained. It was discovered that the inner tube seal at the false sidewall/model endplate interface was the only component of the balance bridge significantly comprising the balance. Hence, this seal was permanently removed (see Chapter II). The removing of this seal was justified since there were no discernible differences in either surface pressure measurements or force measurements for the same conditions whether the seal was inflated or deflated, even at $\alpha=9^{\circ}$. Furthermore, tufts placed on the endplates and on the sidewalls in the immediate vicinity revealed no indication of an induced flow through the gap after the seal had been removed. An examination of Figure B-4 reveals that the balance bridge arrangement used in the second calibration provided results as good as the free balance for this static loading situation.

Since all calibrations were performed with the jet off, it was necessary to determine the balance tares resulting from hose pressurization and flux of momentum. The pressurization tares were obtained by sealing the plenum chamber and then pressurizing the system from zero to six pounds per square inch. Balance readings were recorded at each incremental pressure. The results indicated no pressure tares (i.e. no changes in the balance reading) for the lift component. The tares for drag and pitching moment are presented in Figure B-5 and were repeatable to within $\pm 0.1 \mathrm{lbs}$. The following analytical approximations were then made: 


$$
\begin{gathered}
\mathrm{D}_{\mathrm{T}}=0.1 \mathrm{p}_{\text {op }} \\
\mathrm{PM}_{\mathrm{T}}= \begin{cases}0 \quad & \mathrm{p}_{\text {op }}<3.75 \\
.3 \mathrm{p}_{\mathrm{op}}-1.1, & \mathrm{p}_{\mathrm{op}} \geq 3.75\end{cases}
\end{gathered}
$$

where $p_{o p}$ is the numerical value of the plenum chamber stagnation pressure in pounds per square inch. These approximations were considered adequate in light of the reading uncertainty $( \pm 0.1$ pounds or foot--pounds) for these small loads.

The determination of the momentum tares was more difficult. To accomplish this, it was necessary to construct a pipe (1.5 inches in diameter) that would extend approximately two feet beneath the model which then formed a straight tee with one-foot long arms extending in the upstream and downstream directions. The tee was fabricated to mate with the 1.5 inch diameter nozzle assembly for attachment to the plenum chamber. With this arrangement it was possible to have a jet exit flow which should not have produced any external effect on the balance lift, drag, and pitching moment readings. The momentum tares (after removing the corresponding pressure tares) proved to be negligible in all cases (i.e., the balance readings did not change with the jet on).

Hence, the final equations used in the data reduction were

$$
\begin{aligned}
\mathrm{L} & =.997 \mathrm{~L}_{\mathrm{B}} \\
\mathrm{D} & =.998\left(\mathrm{D}_{\mathrm{B}}-.1 \mathrm{p}_{\mathrm{op}}\right) \\
\mathrm{PM} & = \begin{cases}1.008 \mathrm{PM}_{\mathrm{B}}-.823 \mathrm{D} & , \mathrm{p}_{\mathrm{op}}<3.75 \\
1.008\left(\mathrm{PM}_{\mathrm{B}}-.3 \mathrm{p}_{\mathrm{op}}+1.1\right)-.823 \mathrm{D} \quad, \mathrm{p}_{\mathrm{op}} \geq 3.75\end{cases}
\end{aligned}
$$


where $\mathrm{p}_{\text {op }}$ is the numerical value of the plenum chamber stagnation pressure in psi. These equations are the combined form of the calibration and pressure tare results.

\section{Internal Balance}

Before installing the model in the wind tunnel, it was necessary to determine the characteristics of the internal balance which was used to measure jet thrust. It was hoped to be able to ascertain the pitching and rolling moments in addition to the normal force that the jet would produce on the plenum chamber. For the calibration, the instrumentation set-up and the plenum chamber installation were identical to that of the final model arrangement as described in Chapter II.

A schematic diagram of the plenum chamber showing the assigned locations of the pitching and rolling axis is shown in Figure B-6. Assuming the strain gage outputs are linear allows the following equations to be written for the balance readout,

$$
\begin{aligned}
& F_{N}=\frac{\partial F_{N}}{\partial N} N+\frac{\partial F_{N}}{\partial R} R+\frac{\partial F_{N}}{\partial P} P \\
& F_{R}=\frac{\partial F_{R}}{\partial N} N+\frac{\partial F_{R}}{\partial R} R+\frac{\partial F_{R}}{\partial P} P \\
& F_{P}=\frac{\partial F_{P}}{\partial N} N+\frac{\partial F_{P}}{\partial R} R+\frac{\partial F_{P}}{\partial P} P,
\end{aligned}
$$

where

$$
\mathrm{F}_{\mathrm{N}}=\mathrm{E}_{1}+\mathrm{E}_{2}+\mathrm{E}_{3}+\mathrm{E}_{4}
$$




$$
\begin{aligned}
& \mathrm{F}_{\mathrm{R}}=\left(\mathrm{E}_{1}+\mathrm{E}_{2}\right)-\left(\mathrm{E}_{3}+\mathrm{E}_{4}\right) \\
& \mathrm{F}_{\mathrm{P}}=\left(\mathrm{E}_{1}+\mathrm{E}_{4}\right)-\left(\mathrm{E}_{2}+\mathrm{E}_{3}\right) .
\end{aligned}
$$

The goal of the calibration was then to determine the partial derivatives in the above equations. This was accomplished by applying static loads of $1.32,2.64$, 3.96, and 5.28 pounds in the positive thrust direction at each of the nine load points shown in Figure B-6 and reading (using the computer) the strain gage outputs. These results were then used to compute the partial derivatives through a modification of the method of Reference 37. The modification consisted of using a least squares straight line fit to determine slopes in lieu of the recommended graphical technique. The results of this procedure were

$$
\begin{aligned}
& \mathrm{F}_{\mathrm{N}}=3.349 \mathrm{~N}-.0143 \mathrm{R}+.0173 \mathrm{P} \\
& \mathrm{F}_{\mathrm{R}}=-.0092 \mathrm{~N}+.3136 \mathrm{R}+.0215 \mathrm{P} \\
& \mathrm{F}_{\mathrm{P}}=.4173 \mathrm{~N}+.0242 \mathrm{R}+.0710 \mathrm{P}
\end{aligned}
$$

Hence, given the output of the four bridges, an inversion of the above matrix yields the normal force and pitching and rolling moments.

An initial check on this calibration was provided by statically loading the plenum chamber at the central load point. The result for the normal force output is presented in Figure B-7 and was considered to be satisfactory. The results for the pitching and rolling moment were disappointing in that they were grossly inaccurate and these measurements could not be made. The reason for this is thought 
to be the non-repeatable coupling of strain gage bridge pairs one-two and threefour. That is, these pairs were mounted to the same "floating" rib (see Chapter II) and attempts to measure differences in their outputs were made impossible by the large degree of coupling resulting from the rib rotation.

A verification of the internal balance system calibration was made by measuring actual jet thrust with the wing mounted in the tunnel and a zero crossflow velocity. This was done for the range of thrust levels encountered during actual testing using the 1.5 inch jet at the 45 percent chord exit location with the dental dam seal in place. The results were compared with theoretical thrust calculations using

$$
T=2 q_{j} A_{j}
$$

where $q_{j}$ was determined using the method described in Appendix A. The results are given in Figure B-8 and were considered acceptable. Note that the largest deviation in Figure B- 8 occurred for the lowest thrust level. This discrepancy was attributed to the effects of the dental dam seal which was not installed for the static calibration.

\section{Pressure Measurement System}

The variable capacitance transducers and the associated electronics used to measure surface pressures (see Chapter I) were calibrated before and after the wind tunnel testing. A dead-weight tester manufactured by Consolidated Electrodynamics Corporation (Type 6-201-0001) was used to supply a reference pressure. This tester is a primary laboratory standard and is stated to be accurate within 
0.025 percent of reading.

For the calibration, the pressure system was identical to that of the actual testing arrangement. A modification of the program for the Hewlett-Packard computer using the same logic as that for acquiring the pressure readings was also used. The results of the calibration for the four $1000 \mathrm{~mm}$ full scale Barocel transducers is presented in Figure B-9. It was necessary to use the $1000 \mathrm{~mm}$ full scale transducers on all channels since surface static pressures in excess of $10 \mathrm{~mm}$ of $\mathrm{Hg}$ which was the maximum range of the only other transducers available in the laboratory over the reference ambient pressures frequently occurred. The range of the calibration (0-20 $\mathrm{mm}$ of $\mathrm{Hg}$ ) covered the vast majority of pressure differences which were measured. However, special care in the calibration was taken to assure that the $1000 \mathrm{~mm}$ full scale transducers were accurate for pressure differentials less than five $\mathrm{mm}$ of $\mathrm{Hg}$.

The results presented in Figure B-9 indicated essentially identical results for the two calibrations. The maximum deviation in slope for any of the Barocel transducers is seen to be about one percent. Agreement in the small pressure differential range is also seen to be good. These results were considered satisfactory and corrections due to slope deviations were considered to be so small that they were not included in the data reduction.

It should also be noted that a separate Barocel transducer ( 7.5 psi full scale) was used to monitor plenum stagnation pressure. This eliminated having to read large pressures (greater than five psi) with the $1000 \mathrm{~mm}$ transducers used for surface pressure measurements which might have resulted in a hysteresis effect. The 
maximum slope deviation of this transducer was also within one percent.

\section{Wind Tunnel Freestream}

The installation of the flat sidewalls in the wind tunnel changed the crosssectional area of the test section, thus necessitating a recalibration of the tunnel freestream flow. Also, flow visualization techniques were employed to check the effectiveness of the two-dimensional wing endplate at the sidewalls.

The freestream calibration consisted of a dynamic pressure survey from wall to wall in the plane of the wind chordline. This was accomplished by installing a bar horizontally through the test section approximately eight inches aft of the balance centerline. A dynamic pressure probe was affixed to a slide assembly on the bar which allowed for manual horizontal traversing. This resulted in the tip of the probe being approximately four inches forward of the eventual model leading edge point. Figure B-10 is a photograph of the probe installation. Pressure measurements were made using an inclined alcohol micromanometer.

The dynamic pressure surveys were made at indicated velocities of 50, 100, and 150 feet per second. The results are presented in Figure B-11 in terms of true airspeed. The only anomaly in this result is a slightly higher velocity on the tunnel south side near the wall and a lower velocity on the north side. (The south side is the outboard of the continuous flow tunnel) This discrepancy, which manifested itself particularly at the higher speeds, was attributed to centrifugal effects which amounted to less than one percent deviation in the transverse uniformity. The results were deemed acceptable.

It was also necessary to determine the piezometer setting as measured on 
a Betz water manometer as a function of freestream dynamic pressure so that test section velocities could be set by the tunnel operator. For this purpose, the probe was set at the tunnel centerline and dynamic pressure was measured. The result is presented in Figure B-12 where again the straight line was determined from a least squares fit.

After the model was installed in the tunnel, a tuft study was made of the flow field in the region of the model endplates. This study indicated uniform and well behaved streamlines for all areas forward of the model centerline. However, with the model at angle of attack, a locally separated region occurred on the sidewalls in an area aft and slightly above the model. A slight downflow on the sidewalls aft and below the model was also noticed for this condition. Neither of these effects were considered serious. The tuft study did not reveal any flow through the endplate/sidewall interface even with the inner tube seal removed. 


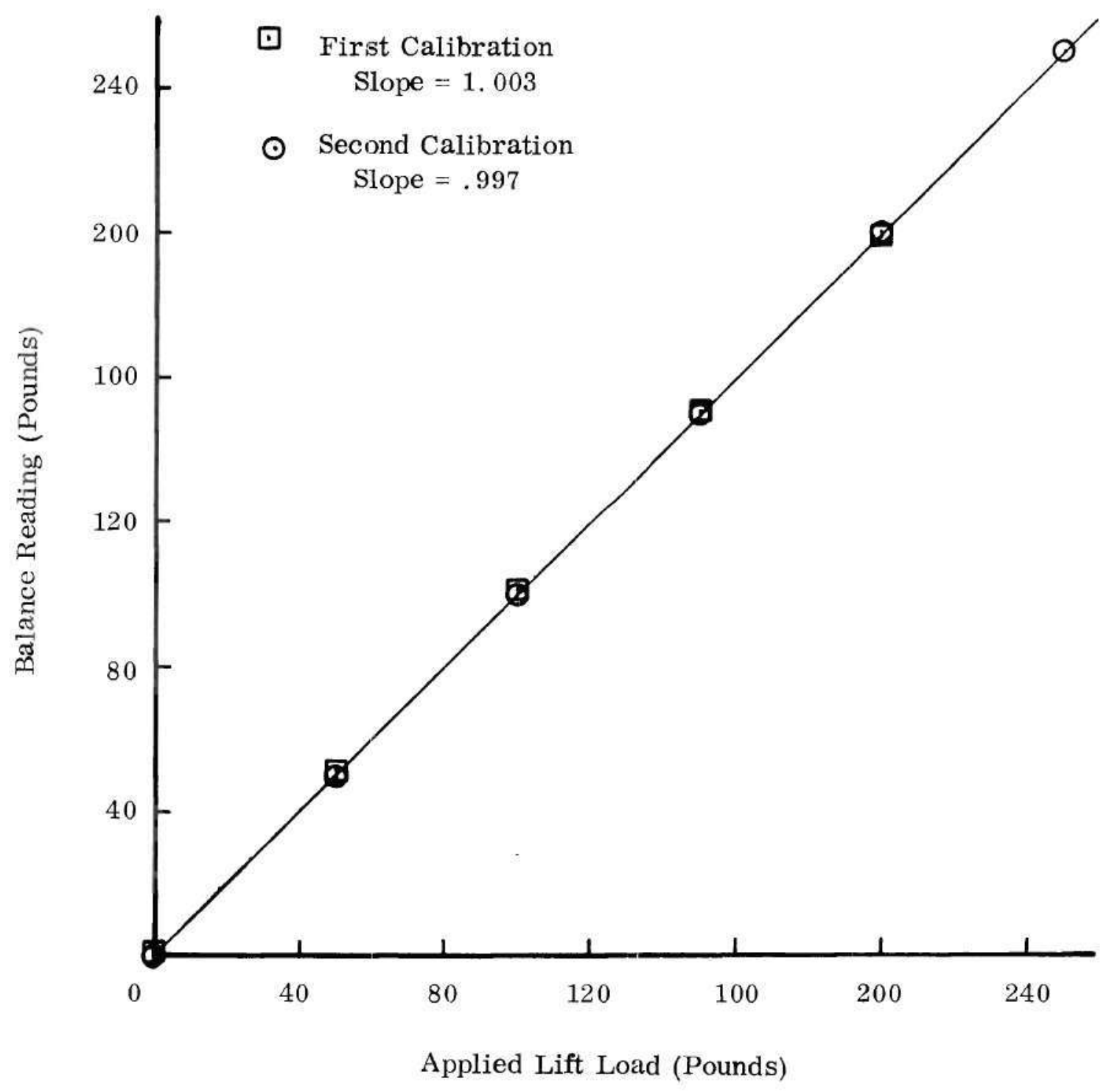

Figure B-1. Comparigon of First and Second Balance Calibrations for the Lift Component. 


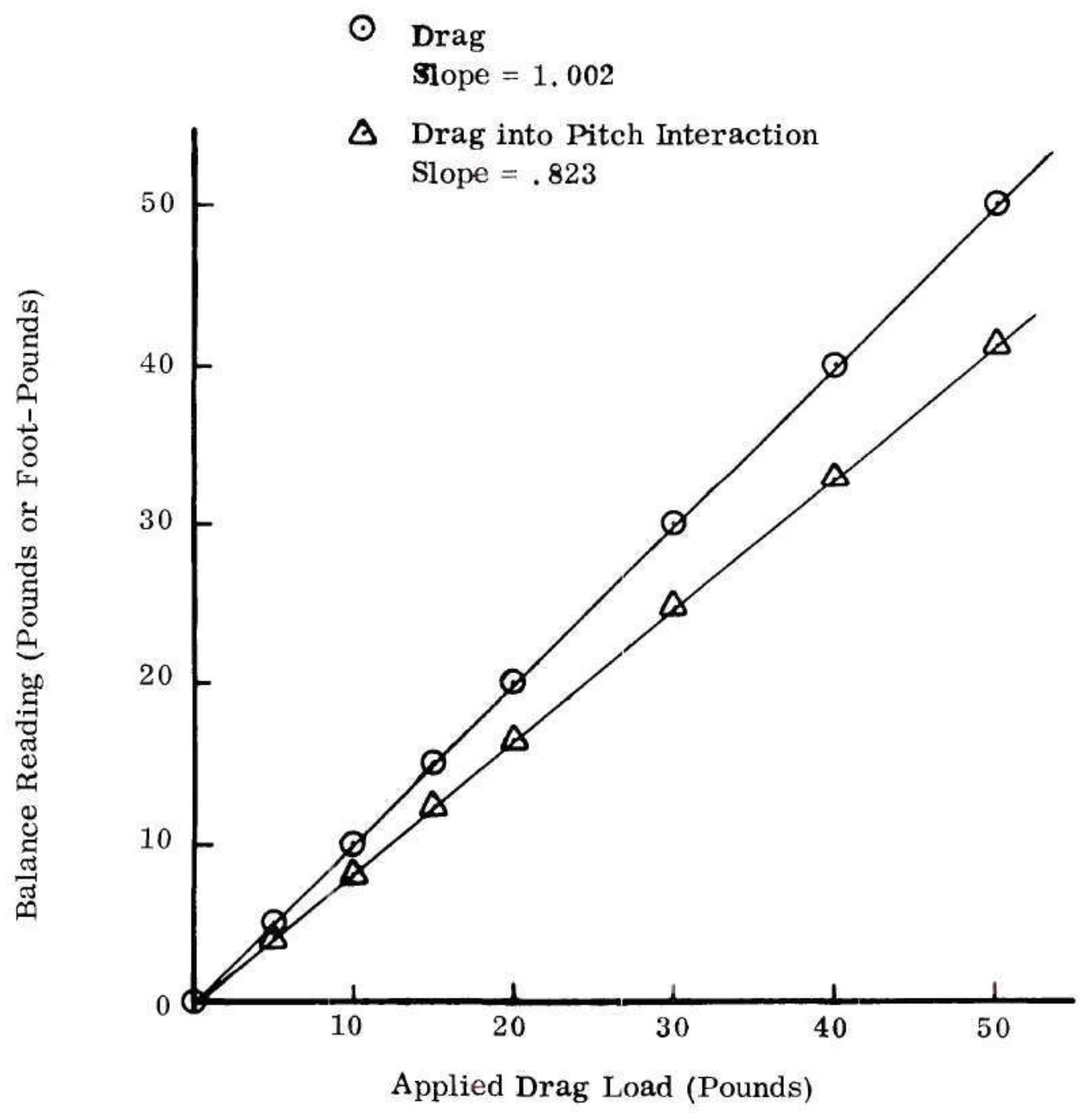

Figure B-2. Balance Calibrations for Drag and Drag Into Pitching Moment Interaction. 


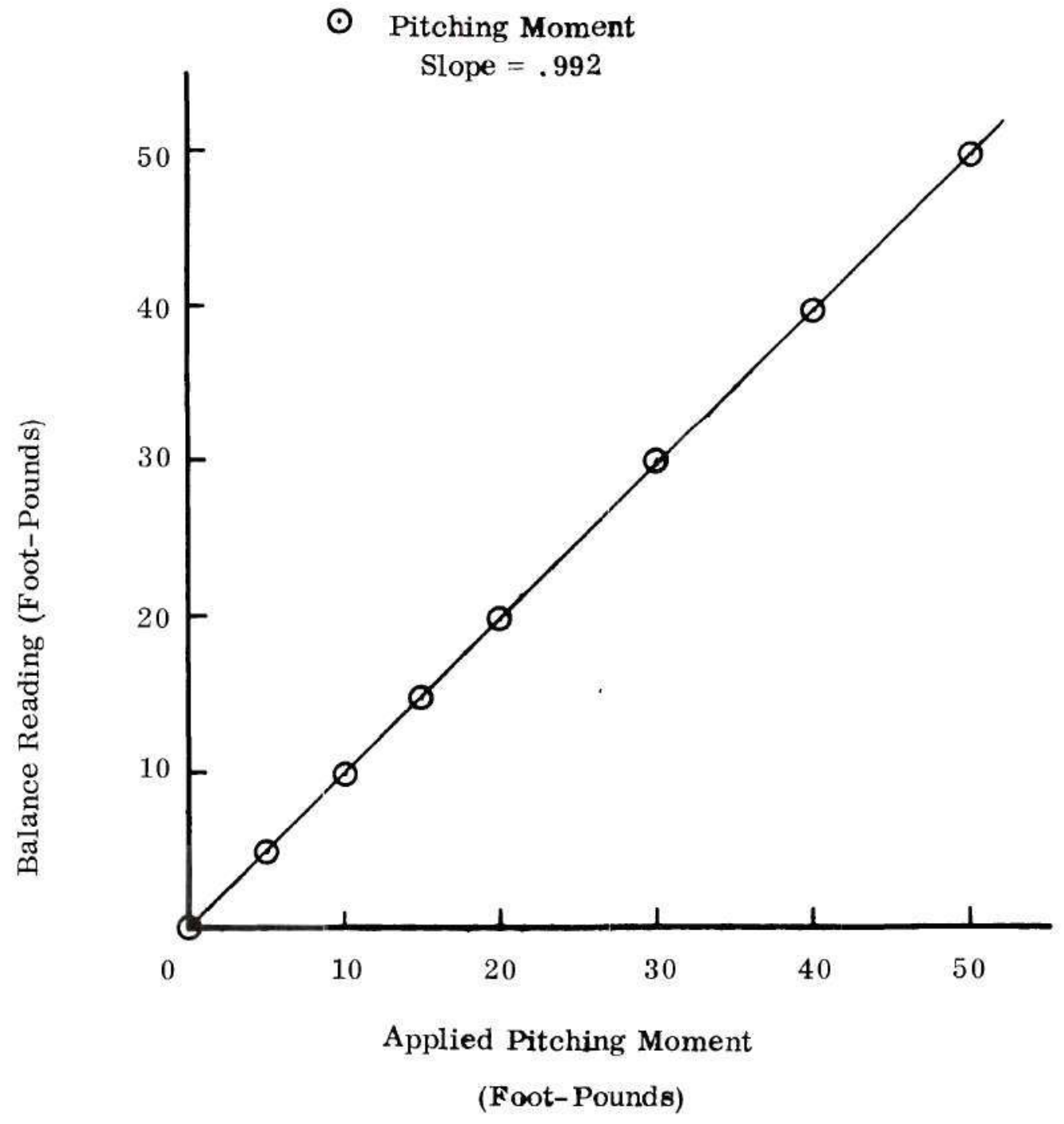

Figure B-3. Balance Calibration for Pitching Moment. 


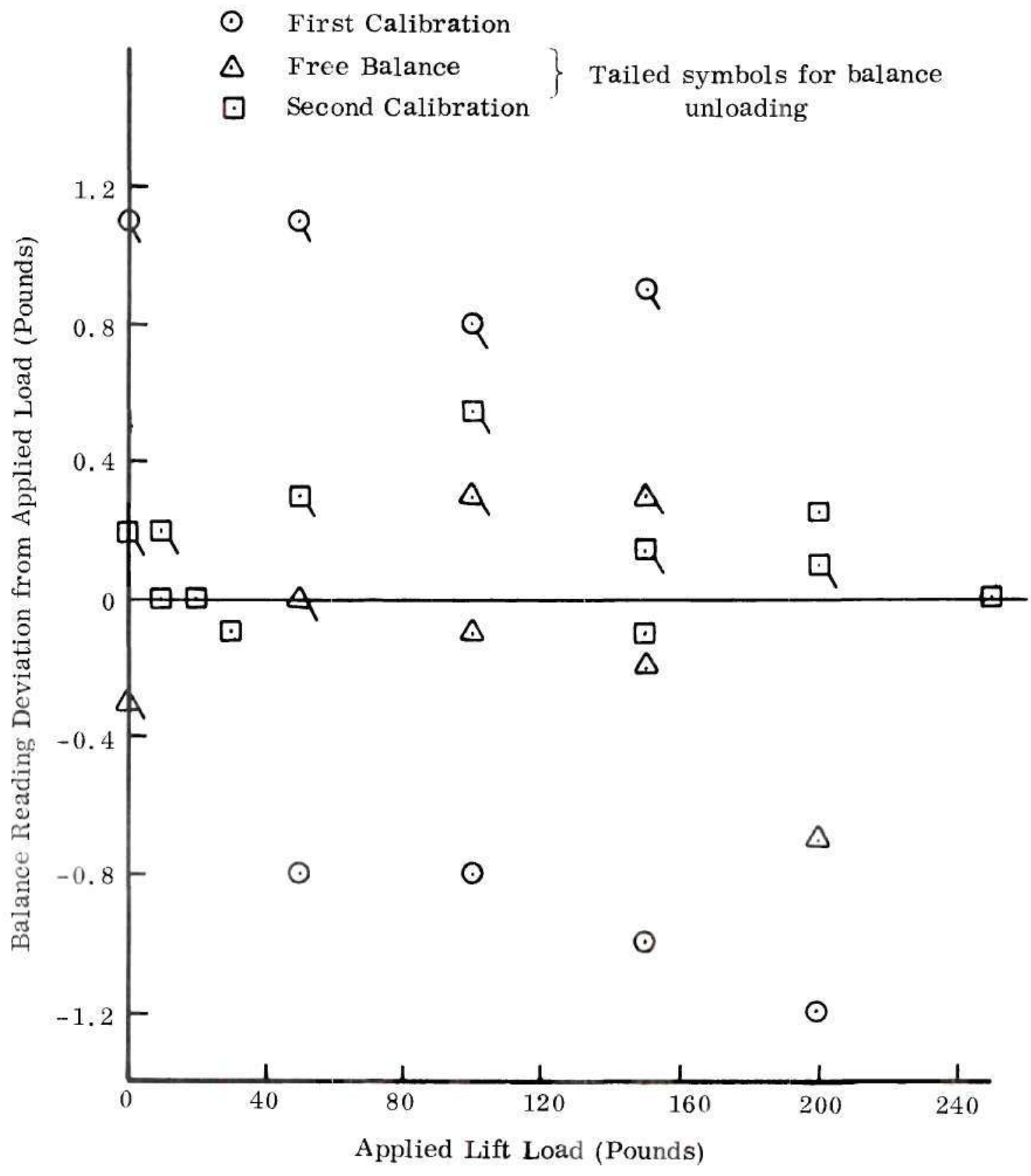

Figure B-4. Comparison of Lift Calibration for Different Balance Bridging Arrangements. 


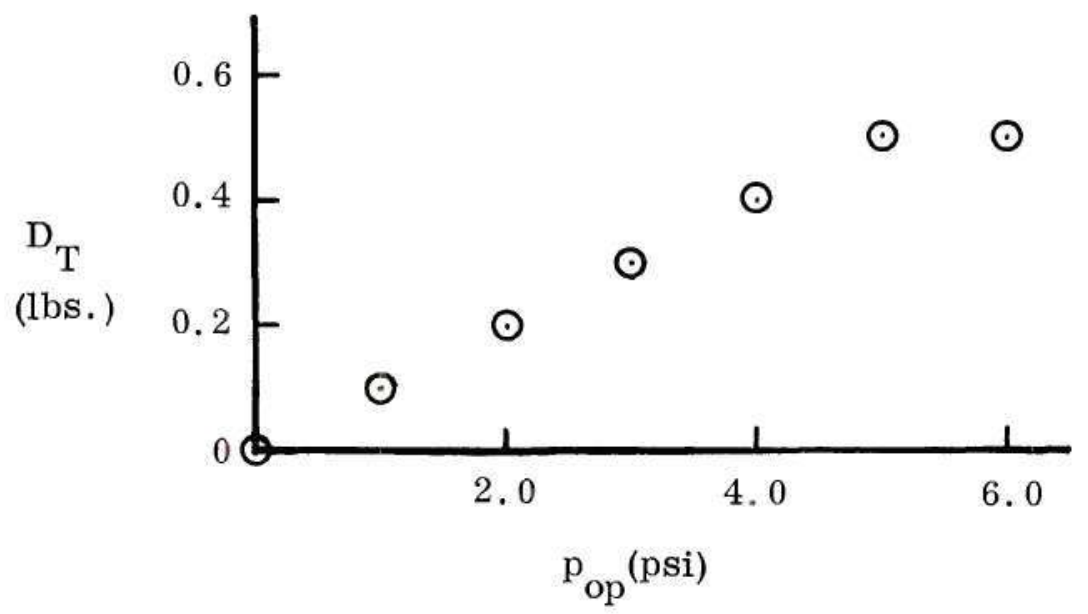

(a) Drag Tare

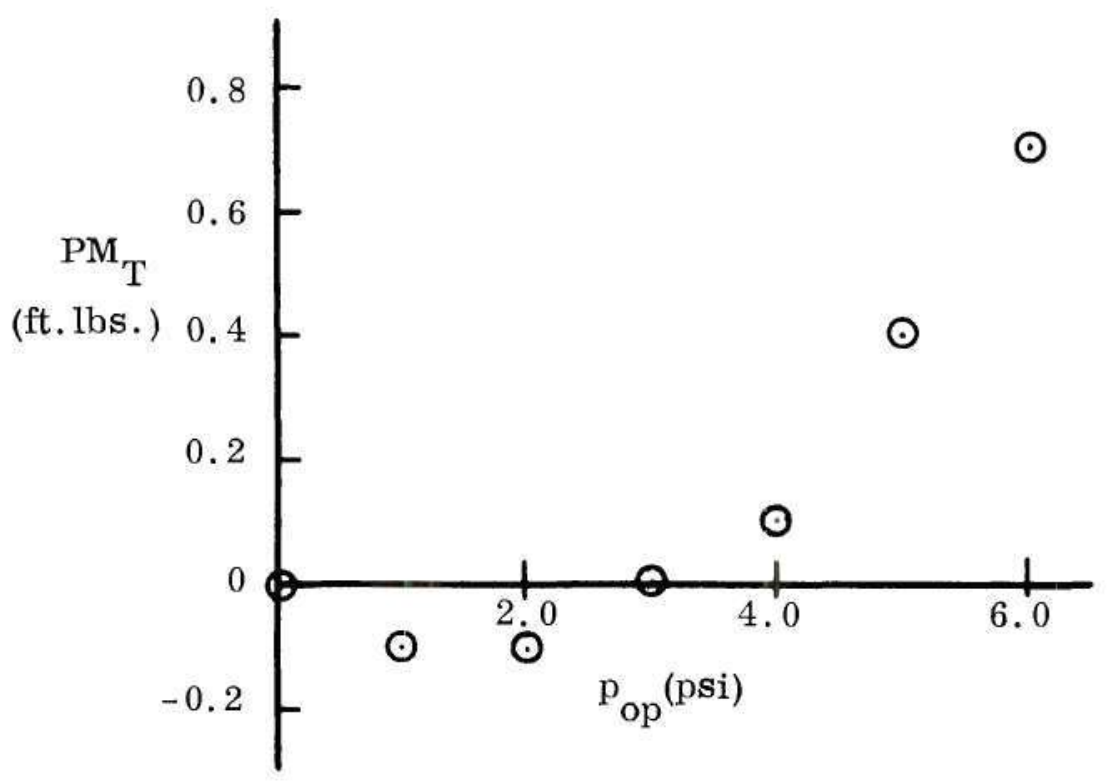

(b) Pitching Moment Tare

Figure B-5. Balance Tares Resulting from

System Pressurization. 
VIEW LOOKING DOWN

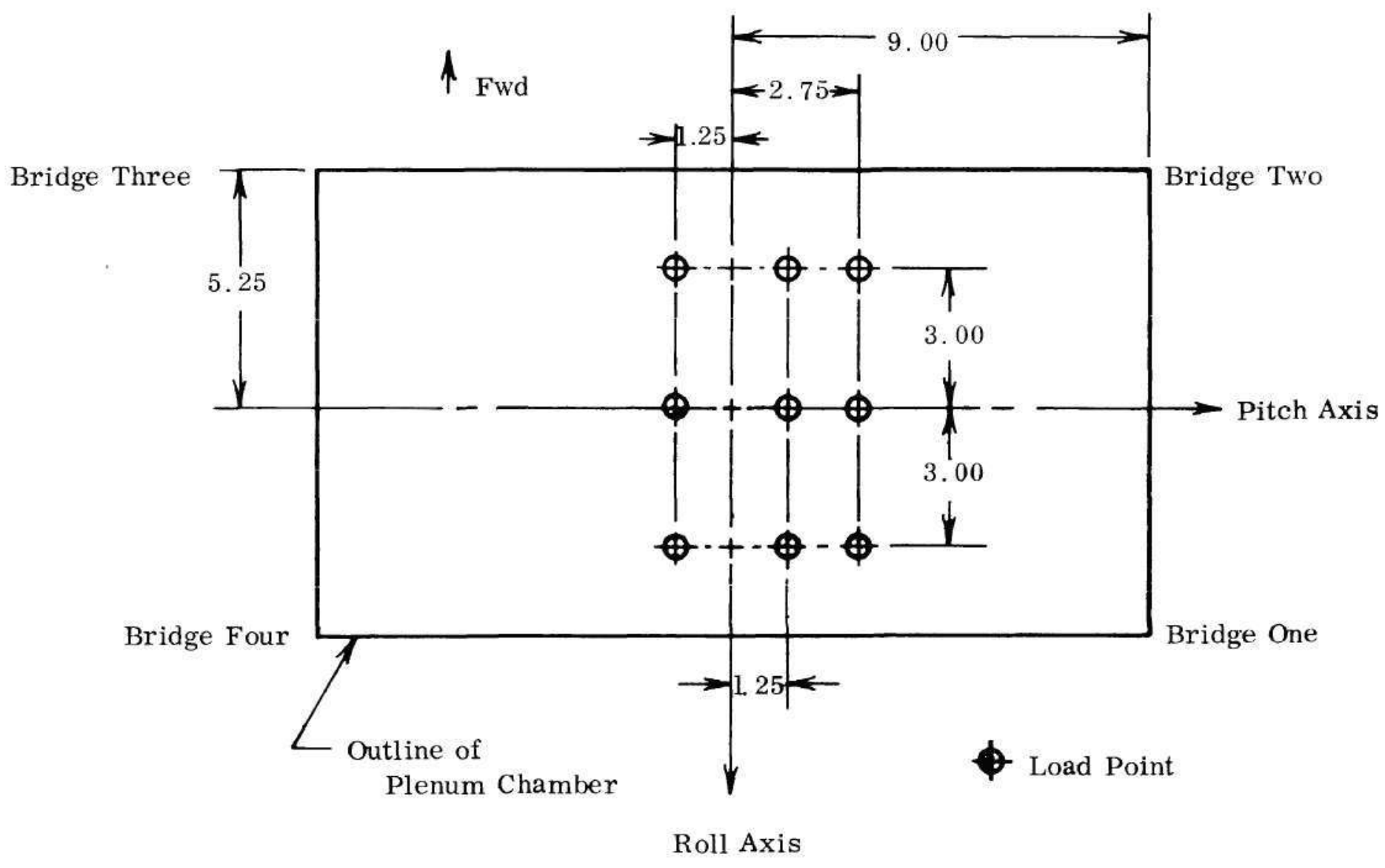

Figure B-6. Schematic Diagram of Plenum Chamber Showing Calibration Axes and Location of Load Points. 


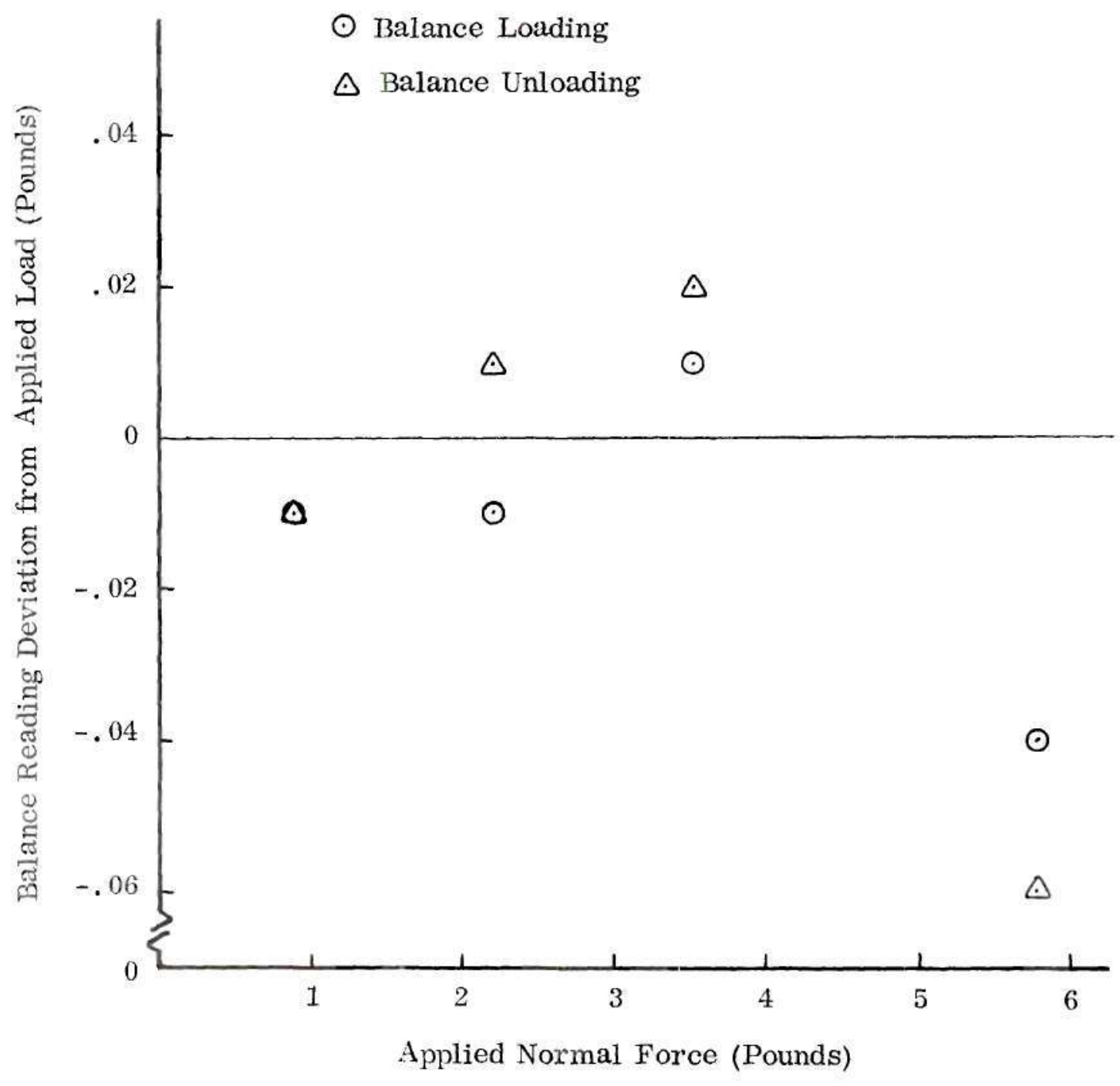

Figure B-7. Internal Balance Normal Force Output in Response to a Static Loading. 


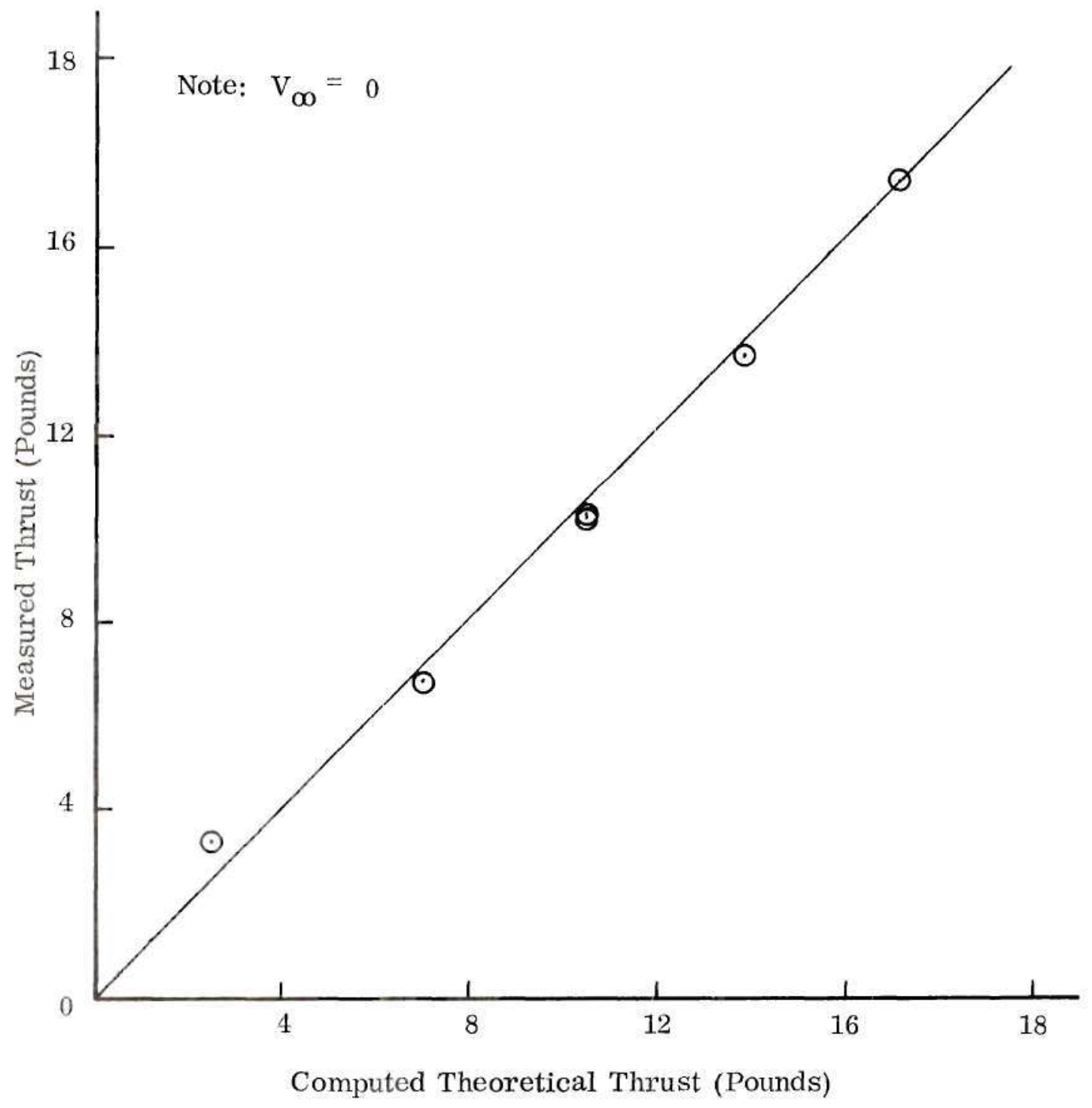

Figure B-8. Thrust as Measured on Internal Balance System Compared with Theoretical Values. 
Transducer Channel 1

Serial No. 1051

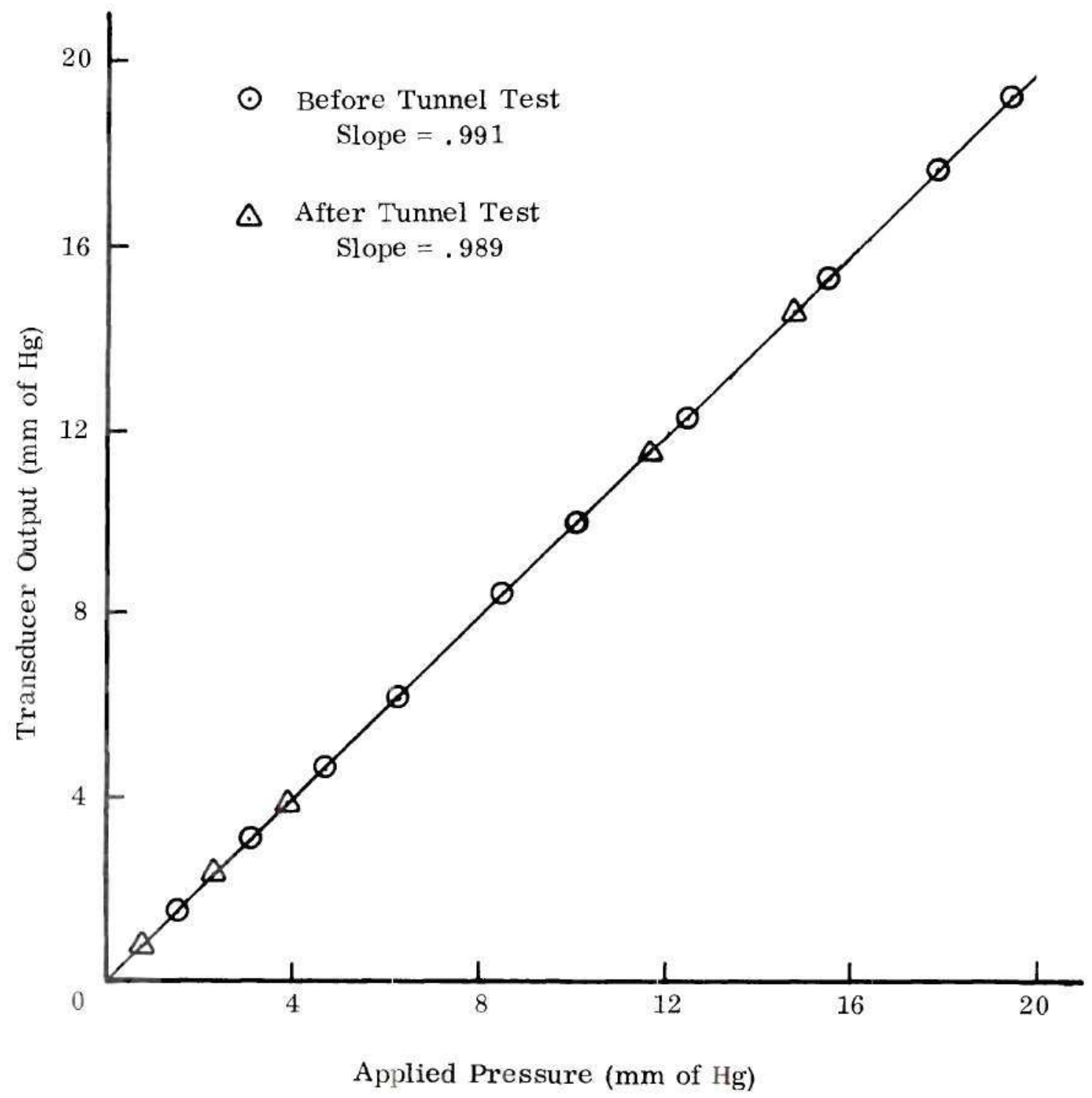

Figure B-9a. Calibration of Pressure Measurement System Against the Dead-Weight Tester. 
Transducer Channel 2

Serial No. 1606

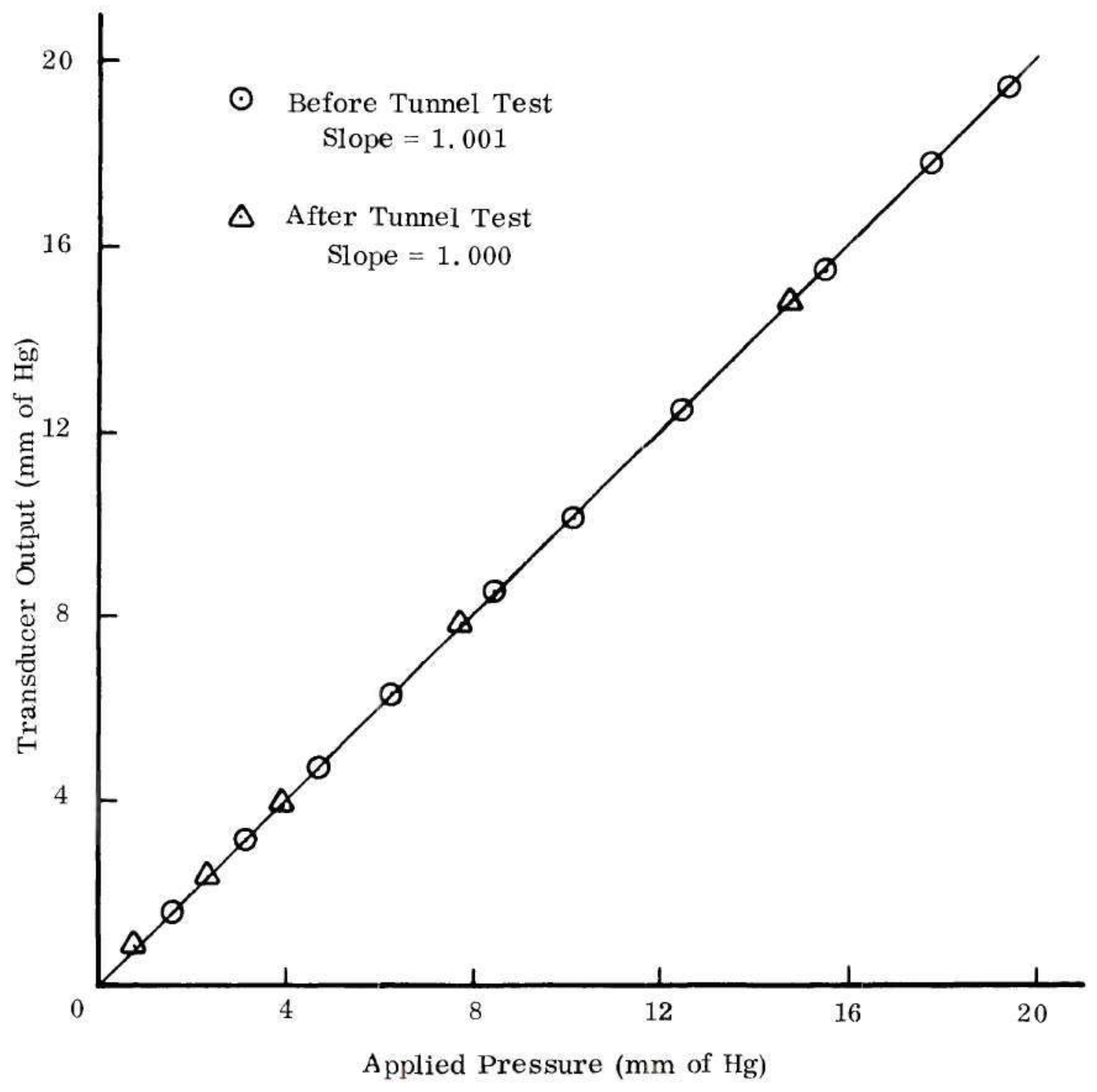

Figure B-9b. Calibration of Pressure Measurement System Against the Dead-Weight Tester. 
Transducer Channel 3

Serial No. 1363

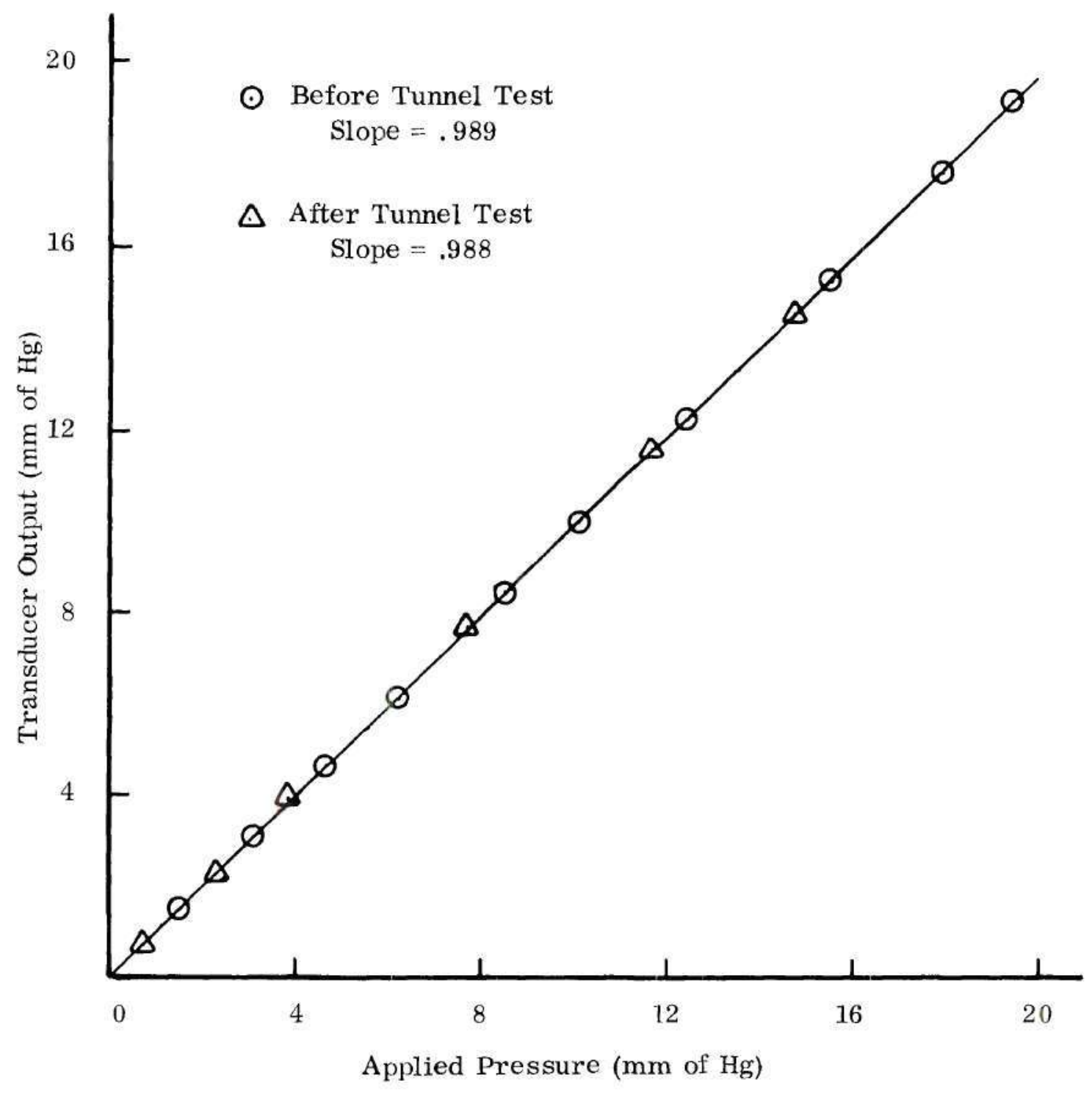

Figure B-9c. Calibration of Pressure Measurement System

Against the Dead-Weight Tester. 
Transducer: Channel 4

Serial No. 1642

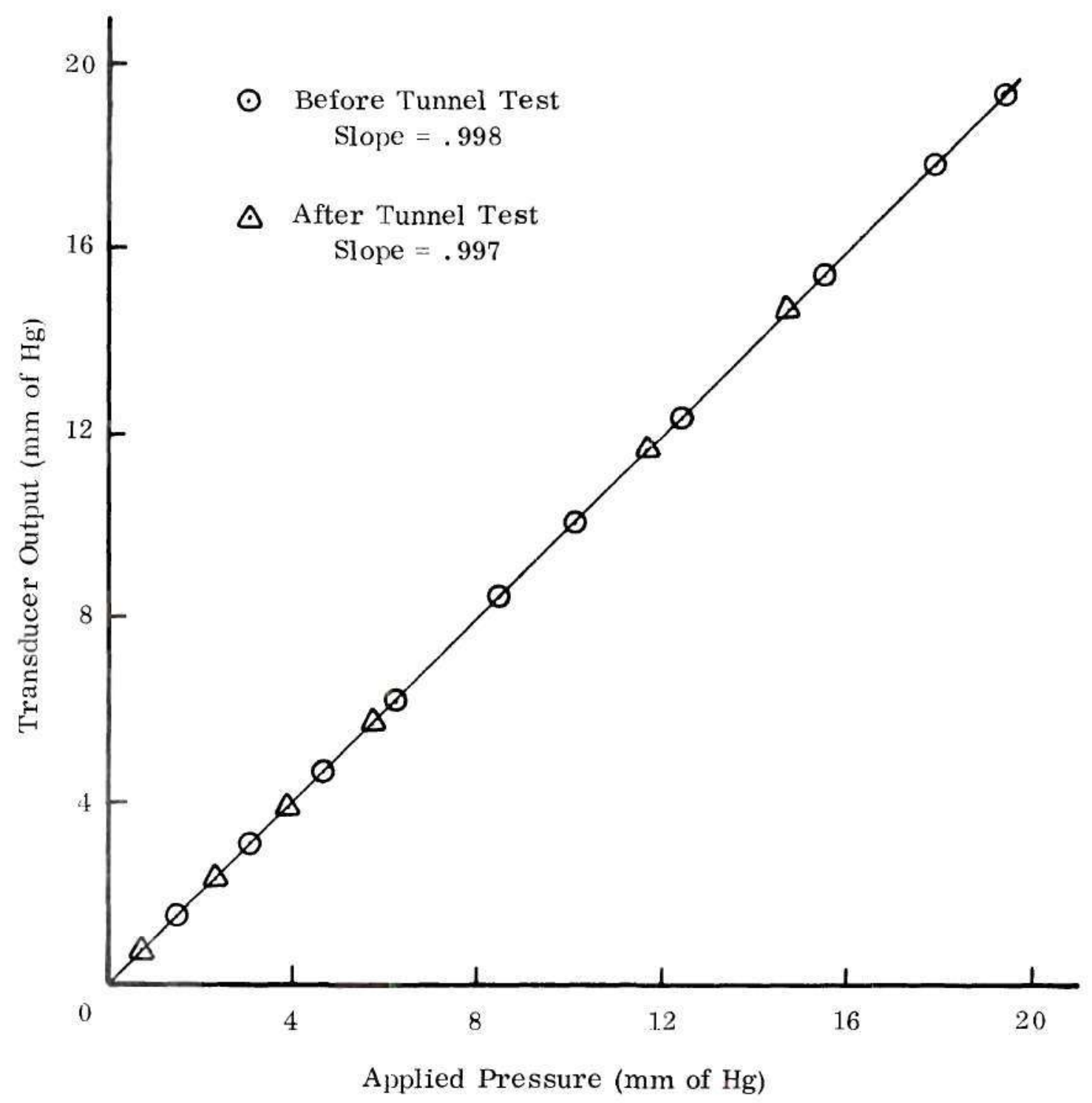

Figure B-9d. Calibration of Pressure Measurement System

Against the Dead-Weight Tester. 


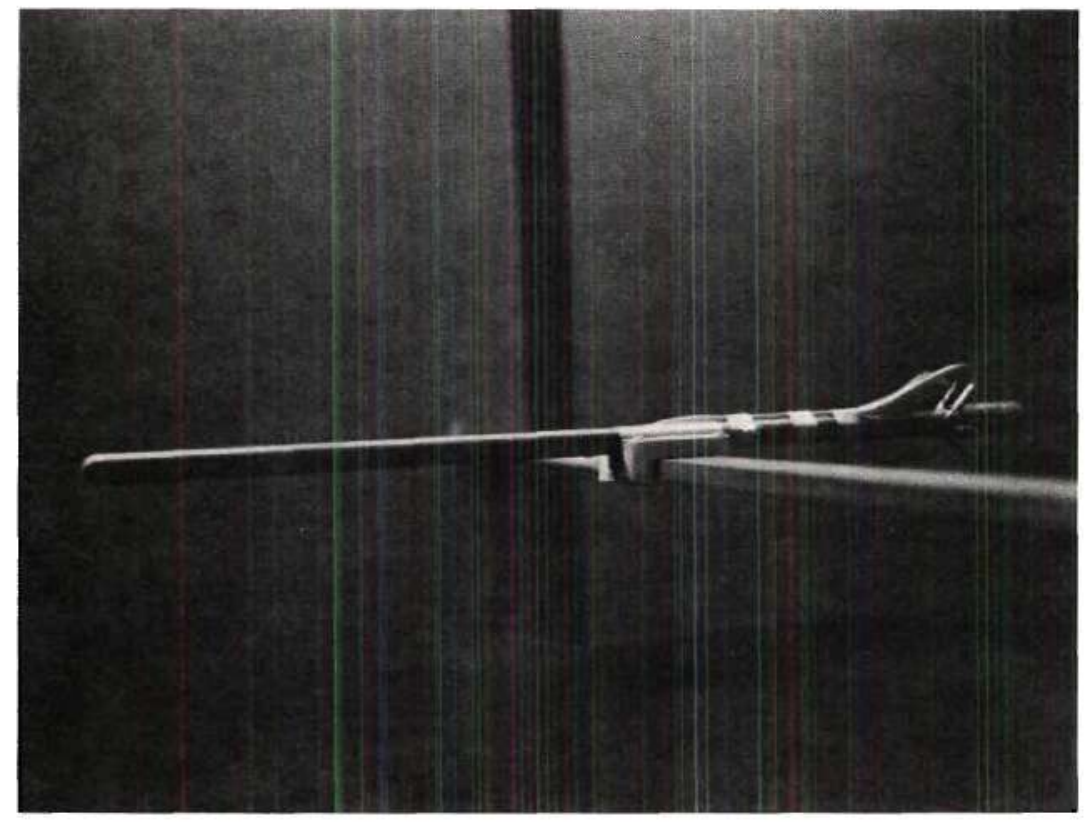

Figure B-10. Wind Tunnel Freestream Dynamic Pressure Probe Arrangement. 


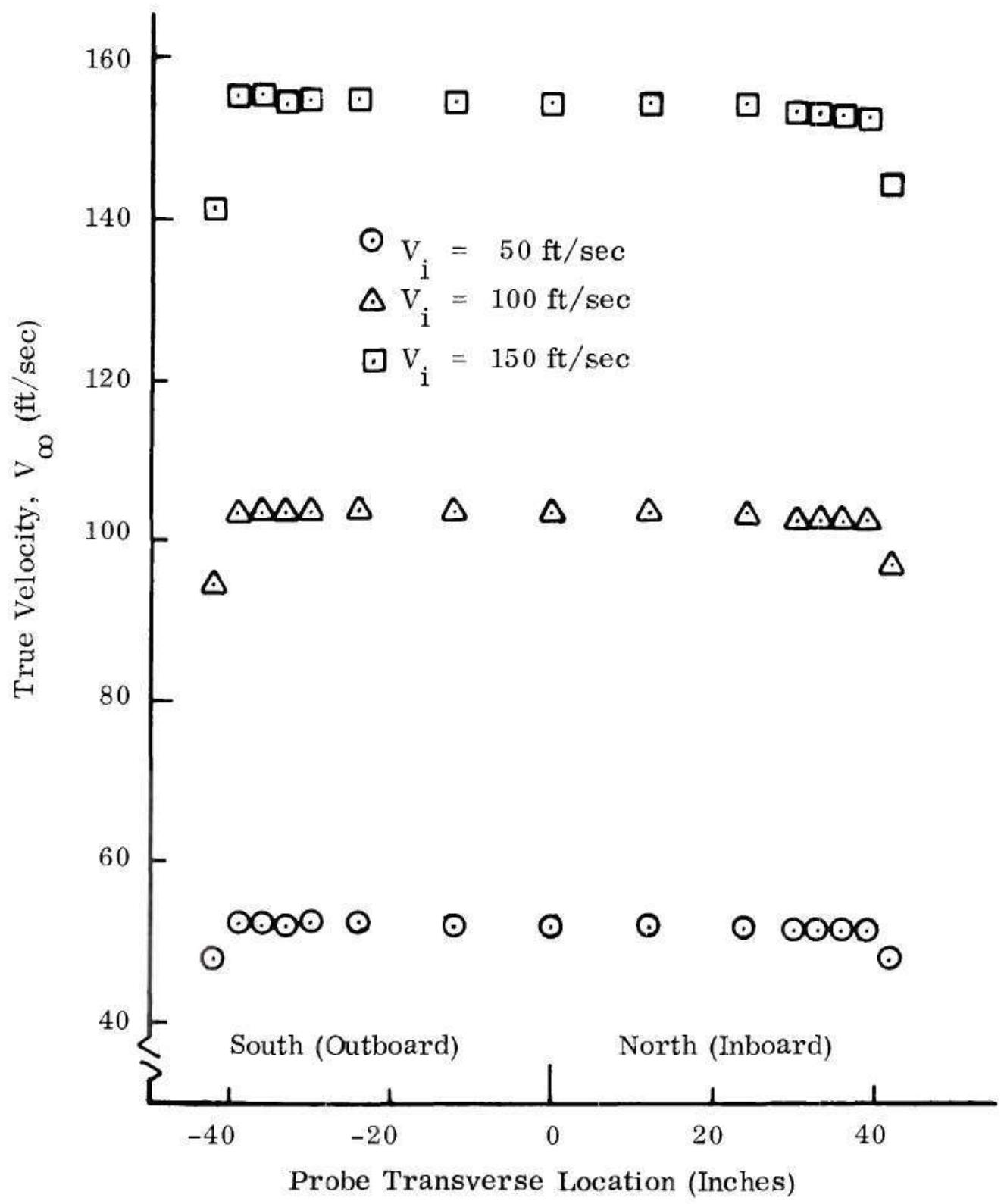

Figure B-11. Results of Dynamic Pressure Survey

of Wind Tunnel Freestream. 


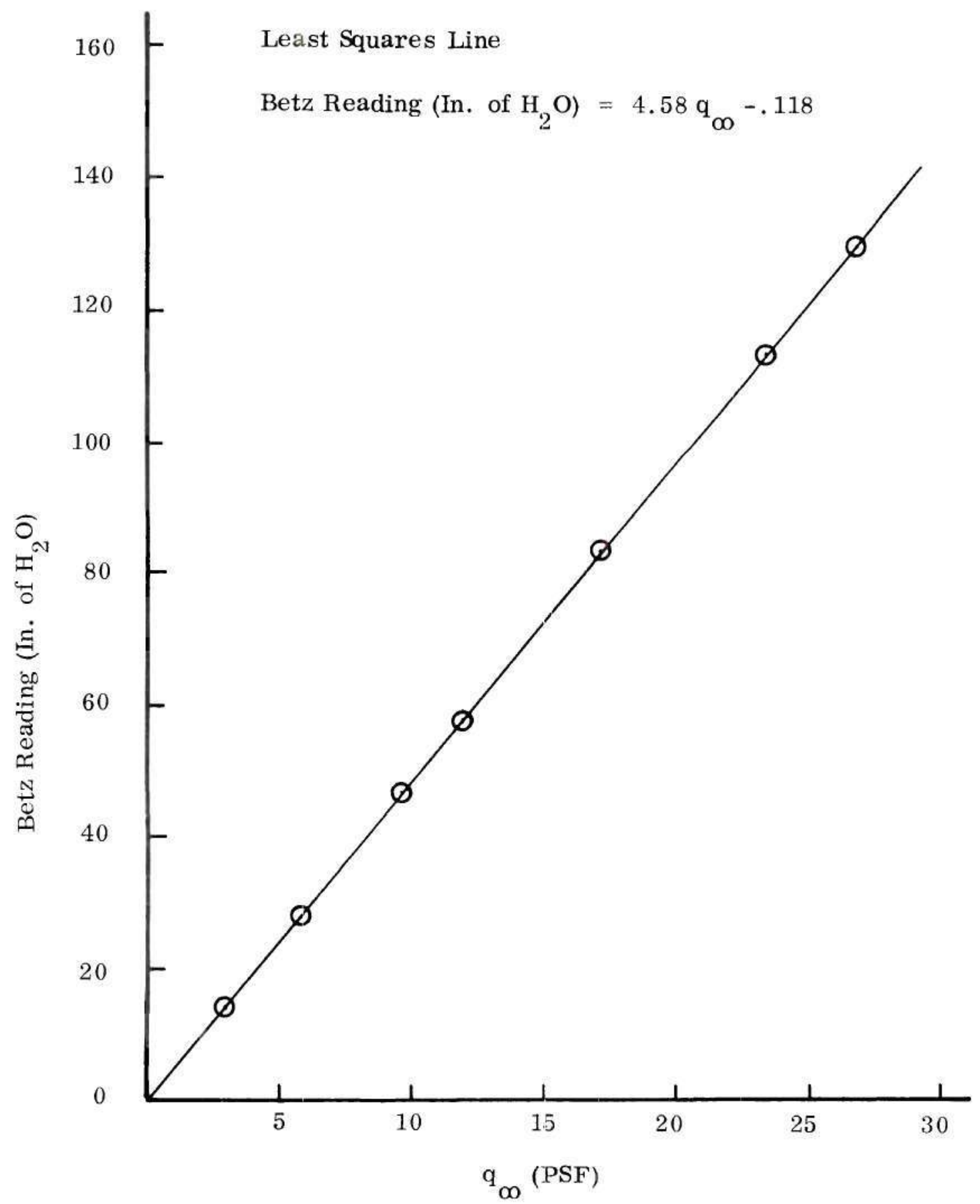

Figure B-12. Piezometer Setting for Desired

Freestream Dynamic Pressure. 


\section{APPENDIX C}

\section{INTERFERENCE SURFACE PRESSURE DISTRIBUTIONS}

This appendix contains the contour plots of the interference pressure distributions on the wing lower surface for the conditions investigated in the experiment. Interference pressure distributions on the upper surface for some cases of interest are also presented.

The interference pressure coefficient was computed as

$$
\Delta \mathrm{C}_{\mathrm{p}}=\mathrm{C}_{\mathrm{p} \text { jet-on }}-\mathrm{C}_{\mathrm{p} \text { jet-off }}
$$

where

$$
\mathrm{C}_{\mathrm{p}}=\frac{\mathrm{p}_{\mathrm{s}}-\mathrm{p}_{\infty}}{\mathrm{q}_{\infty}}
$$

Several surface pressure taps did not have identical locations for tests with the jet on and the jet off. (This situation occurred for the pressure taps located on the interchangeable wing skin panel which contained the jet exit.) For these cases, an average chordwise pressure distribution was computed by utilizing four spanwise stations near the wing centerline from the appropriate jet-off data. The jet-off pressure coefficient for the $\Delta \mathrm{C}_{\mathrm{p}}$ calculation was then obtained for the tap location in question by using an Aitken second order interpolation. Sample computer printouts of the pressure distribution and the interference pressure distribution for both wing surfaces are given in Tables $\mathrm{C}-1$. 
Table C-2 is a summary of the configurations and conditions for which pressure measurements were recorded. The appropriate figure number for each case is also given in Table C-2. Note that upper surface interference pressure distributions are presented for the 3.0 inch jet exit diameter. Only minor changes in the upper surface pressure distribution were observed in the machine plots for jet-on operation with the 1.5 inch diameter jet exits and hence this data is not presented.

Since the contour pressure distribution plots were all made by machine, some suggestions on interpreting these plots are in order. The computer program was coded such that the contours would be shown for every 0.10 increment in interference pressure coefficient. In addition, every 0.50 increment is shown as a double line and, if space permits, is labeled. Except for those double width lines, contour levels closer than 0.01 inches (on the plotted page) are suppressed. With a little practice, the values of each contour level on the interference surface pressure distribution can easily be determined if the above rules are kept in mind.

As a further interpretation aid, regions of positive and negative interference pressures (as bounded by $\Delta \mathrm{C}_{\mathrm{p}}=0$ ) are indicated on some plots. Unless otherwise noted, positive contour levels become larger counterclockwise with reference to the jet exit from the $\Delta \mathrm{C}_{\mathrm{p}}=0$ line and negative contour levels become smaller (more negative) clockwise.

It should also be noted that the interference surface pressure contours were drawn to scale. That is, the horizontal extent corresponds to the 15.37 inch wing chord and the vertical extent to the spanwise section from $y=0$ inches to $y=18.0$ inches. 
Table C-1a. Sample Computer Printout of Surface

Pressure Coefficients.

\begin{tabular}{|c|c|c|c|c|c|c|c|c|}
\hline & $\begin{array}{l}\text { JET EXIT } \\
\text { ANGLE OF }\end{array}$ & $\begin{array}{l}\text { JET IO } \\
\text { LOCATION = } \\
\text { ATTACK = }\end{array}$ & $\begin{array}{l}\text { SSUING FF } \\
=.65 \mathrm{PC} \\
6.0 \mathrm{DEGF}\end{array}$ & $\begin{array}{l}\text { ROM A WING } \\
\text { T. CHORA } \\
\text { REES }\end{array}$ & $\begin{array}{l}\text { CROSSFLO } \\
\text { JET DIAME } \\
\text { VELOCITY }\end{array}$ & $\begin{array}{l}\text { W } \\
\text { IETER/C } \\
\text { RATIO }\end{array}$ & $\begin{array}{l}\text { RUiv } \\
\text { ORU }= \\
=4.0\end{array}$ & $\begin{array}{l}\text { NO. } \\
10\end{array}$ \\
\hline$x$ & $Y$ & $\begin{array}{l}\text { UPPER } \\
C P\end{array}$ & $\underset{x}{\text { SURFACE }}$ & $\underset{Y}{\text { PRESSURE }}$ & $\begin{array}{l}\text { DISTRIBUTION } \\
\text { CP }\end{array}$ & $x$ & $Y$ & $C P$ \\
\hline $\begin{array}{r}.00 \\
.70 \\
2.25 \\
3.75 \\
5.25 \\
6.75 \\
8.25 \\
9.75 \\
11.25 \\
12.75 \\
14.25 \\
.00 \\
.70 \\
2.25 \\
3.75 \\
5.25\end{array}$ & $\begin{array}{l}.00 \\
.00 \\
.00 \\
.00 \\
.00 \\
.00 \\
.00 \\
.00 \\
.00 \\
.00 \\
.00 \\
1.00 \\
2.00 \\
2.00 \\
2.00 \\
2.00\end{array}$ & $\begin{array}{r}.343 \\
-2.036 \\
-1.739 \\
-1.207 \\
-.907 \\
-.742 \\
-.014 \\
-.427 \\
-.226 \\
-.071 \\
.032 \\
.369 \\
-1.846 \\
-1.749 \\
-1.224 \\
-.916\end{array}$ & $\begin{array}{r}6.75 \\
9.25 \\
9.75 \\
11.25 \\
12.75 \\
.00 \\
.70 \\
2.25 \\
3.75 \\
5.25 \\
6.75 \\
9.25 \\
9.75 \\
11.25 \\
12.75 \\
14.25\end{array}$ & $\begin{array}{l}2.00 \\
2.00 \\
2.00 \\
2.00 \\
2.00 \\
3.00 \\
4.00 \\
4.00 \\
4.00 \\
4.00 \\
4.00 \\
4.00 \\
4.00 \\
4.00 \\
4.00 \\
4.00\end{array}$ & $\begin{array}{r}-.743 \\
-.591 \\
-.421 \\
-.012 \\
-.063 \\
.337 \\
-1.887 \\
-1.767 \\
-1.222 \\
-.926 \\
-.741 \\
-.588 \\
-.426 \\
-.213 \\
-.061 \\
.037\end{array}$ & $\begin{array}{r}3.75 \\
6.75 \\
9.75 \\
12.75 \\
3.75 \\
6.75 \\
9.75 \\
2.25 \\
3.75 \\
5.25 \\
6.75 \\
8.25 \\
11.25\end{array}$ & $\begin{array}{r}7.00 \\
7.00 \\
7.00 \\
7.00 \\
11.00 \\
11.00 \\
11.00 \\
-2.00 \\
-2.00 \\
-2.00 \\
-2.00 \\
-2.00 \\
-2.00\end{array}$ & $\begin{array}{r}-1.194 \\
-.740 \\
-.409 \\
-.055 \\
-1.201 \\
-.750 \\
-. .386 \\
-1.734 \\
-1.206 \\
-.915 \\
-.732 \\
-.5589 \\
-. .228\end{array}$ \\
\hline
\end{tabular}


Table C-1b. Sample Computer Printout of Surface

Pressure Coefficients

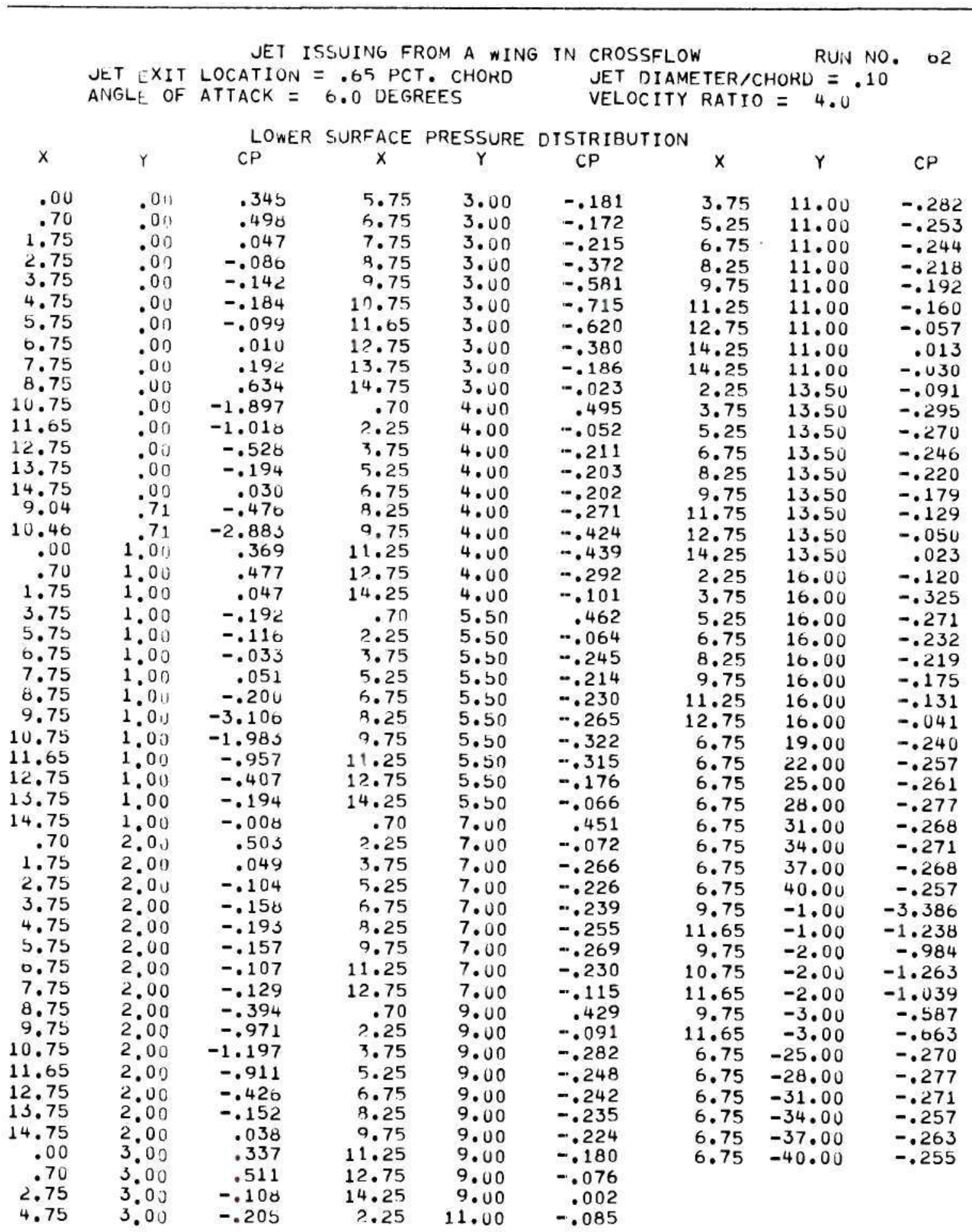


Table C-1c. Sample Computer Printout of Surface

\author{
Pressure Coefficients
}

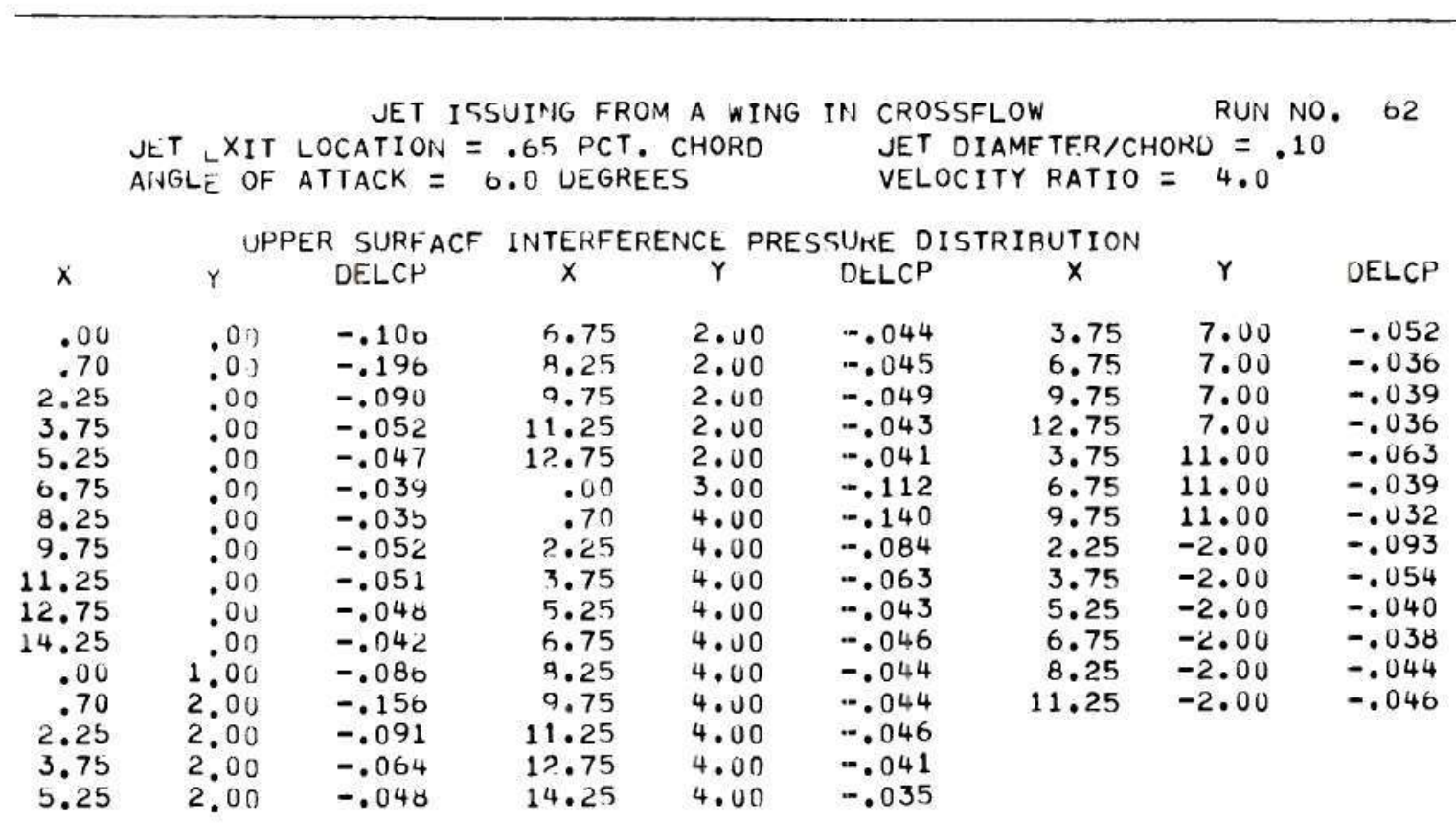


Table C-1d. Sample Computer Printout of Surface

\author{
Pressure Coefficients
}

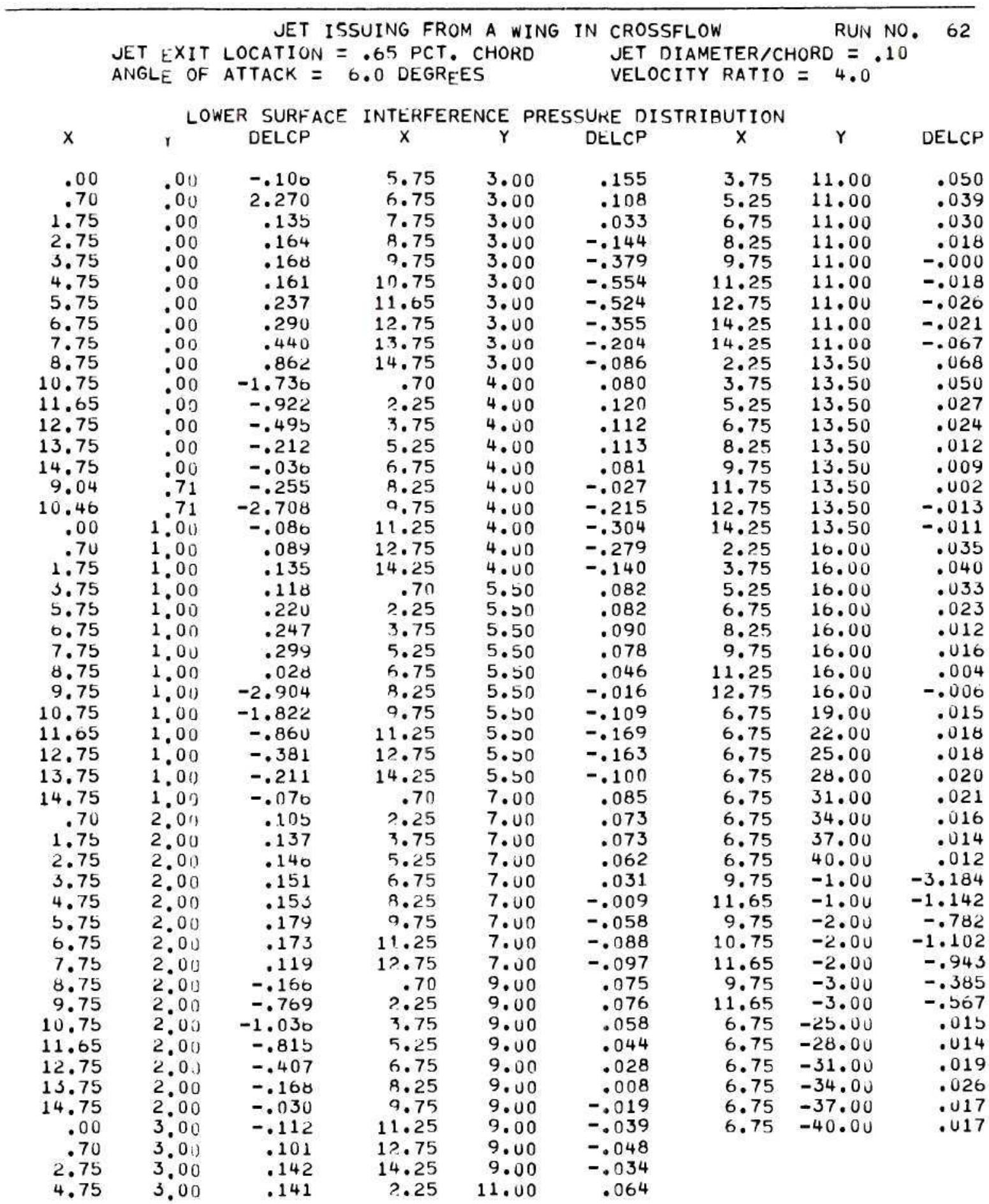


Table C-2. Summary of Interference Surface Pressure Distributions

\begin{tabular}{|c|c|c|c|c|}
\hline $\mathrm{d}_{\mathrm{j}} / \mathrm{c}$ & $x_{j} / c$ & $\alpha$ & $\lambda$ & Figure Number \\
\hline 0.10 & 0.25 & $0^{\circ}$ & 2 & $C-1 a$ \\
\hline 0.10 & 0.25 & $0_{0}^{\circ}$ & 4 & $\mathrm{C}-1 \mathrm{~b}$ \\
\hline 0.10 & 0.25 & $0^{\circ}$ & 8 & $C-1 c$ \\
\hline 0.10 & 0.25 & $0_{0}^{0}$ & 12 & $C-1 d$ \\
\hline 0.10 & 0.25 & $6^{\circ}$ & 2 & $C-1 e$ \\
\hline 0.10 & 0.25 & $6^{\circ}$ & 4 & $C-1 f$ \\
\hline 0.10 & 0.25 & $6^{0}$ & 8 & $C-1 \mathrm{~g}$ \\
\hline 0.10 & 0.25 & $9^{\circ}$ & 2 & $\mathrm{C}-1 \mathrm{~h}$ \\
\hline 0.10 & 0.25 & $9^{\circ}$ & 4 & $C-1 i$ \\
\hline 0.10 & 0.25 & $9^{\circ}$ & 8 & $C-1 j$ \\
\hline 0.10 & 0.45 & $0_{0}^{\circ}$ & 2 & $C-2 a$ \\
\hline 0.10 & 0.45 & $0_{0}^{\circ}$ & 4 & $C-2 b$ \\
\hline 0.10 & 0.45 & $0^{\circ}$ & 8 & $\mathrm{C}-2 \mathrm{c}$ \\
\hline 0.10 & 0.45 & $0^{\circ}$ & 12 & $C-2 d$ \\
\hline 0.10 & 0.45 & $6^{\circ}$ & 2 & $\mathrm{C}-2 \mathrm{e}$ \\
\hline 0.10 & 0.45 & $6_{0}^{0}$ & 4 & $C-2 f$ \\
\hline 0.10 & 0.45 & $6^{\circ}$ & 8 & $C-2 g$ \\
\hline 0.10 & 0.45 & $9^{\circ}$ & 2 & $\mathrm{C}-2 \mathrm{~h}$ \\
\hline 0.10 & 0.45 & $9_{0}^{\circ}$ & 4 & $\mathrm{C}-2 \mathbf{i}$ \\
\hline 0.10 & 0.45 & $9_{0}^{\circ}$ & 8 & $C-2 j$ \\
\hline 0.10 & 0.65 & $0_{0}^{\circ}$ & 2 & $C-3 a$ \\
\hline 0.10 & 0.65 & $0^{\circ}$ & 4 & $C-3 b$ \\
\hline 0.10 & 0.65 & $0^{\circ}$ & 8 & $\mathrm{C}-3 \mathrm{c}$ \\
\hline 0.10 & 0.65 & $0^{\circ}$ & 12 & $C-3 d$ \\
\hline 0.10 & 0.65 & $6^{\circ}$ & 2 & $\mathrm{C}-3 \mathrm{e}$ \\
\hline 0.10 & 0.65 & $6^{\circ}$ & 4 & $C-3 f$ \\
\hline 0.10 & 0.65 & $6_{0}^{\circ}$ & 8 & $\mathrm{C}-3 \mathrm{~g}$ \\
\hline 0.10 & 0.65 & $9_{0}^{\circ}$ & 2 & $\mathrm{C}-3 \mathrm{~h}$ \\
\hline 0.10 & 0.65 & $9_{0}^{\circ}$ & 4 & $\mathrm{C}-3 \mathbf{i}$ \\
\hline 0.10 & 0.65 & 9 & 8 & $\mathrm{C}-3 \mathbf{j}$ \\
\hline 0.20 & 0.45 & $0^{\circ}$ & 4 & $C-4 a, b$ \\
\hline 0.20 & 0.45 & $0^{\circ}$ & 5.9 & C- $4 c, d$ \\
\hline 0.20 & 0.45 & $0_{0}^{0}$ & 8 & $\mathrm{C}-4 \mathrm{e}, \mathrm{f}$ \\
\hline 0.20 & 0.45 & $0_{0}^{\circ}$ & 12 & C-4g, h \\
\hline 0.20 & 0.45 & $6_{0}^{\circ}$ & 4 & $\mathrm{C}-4 \mathrm{i}, \mathrm{j}$ \\
\hline 0.20 & 0.45 & $6^{\circ}$ & 5.9 & $\mathrm{C}-4 \mathrm{k}, 1$ \\
\hline 0.20 & 0.45 & $6^{\circ}$ & 8 & $\mathrm{C}-4 \mathrm{~m}, \mathrm{n}$ \\
\hline
\end{tabular}


Table C-2. (Concluded)

\begin{tabular}{lllll}
\hline $\mathrm{d}_{\mathrm{j}} / \mathrm{c}$ & $\mathrm{x}_{\mathrm{j}} / \mathrm{c}$ & $\alpha$ & $\lambda$ & Figure Number \\
\hline 0.20 & 0.45 & $9^{\mathrm{o}}$ & 4 & $\mathrm{C}-40, \mathrm{p}$ \\
0.20 & 0.45 & $9^{\mathrm{o}}$ & 5.9 & $\mathrm{C}-4 \mathrm{q}, \mathrm{r}$ \\
0.20 & 0.45 & $9^{\mathrm{o}}$ & 8 & $\mathrm{C}-4 \mathrm{~s}, \mathrm{t}$ \\
0.20 & 0.45 & $12^{\mathrm{o}}$ & 4 & $\mathrm{C}-4 \mathrm{u}, \mathrm{v}$ \\
0.20 & 0.45 & $12^{\mathrm{o}}$ & 5.9 & $\mathrm{C}-4 \mathrm{w}, \mathrm{x}$ \\
0.20 & 0.45 & $12^{\mathrm{o}}$ & 8 & $\mathrm{C}-4 \mathrm{y}, \mathrm{z}$ \\
\hline
\end{tabular}




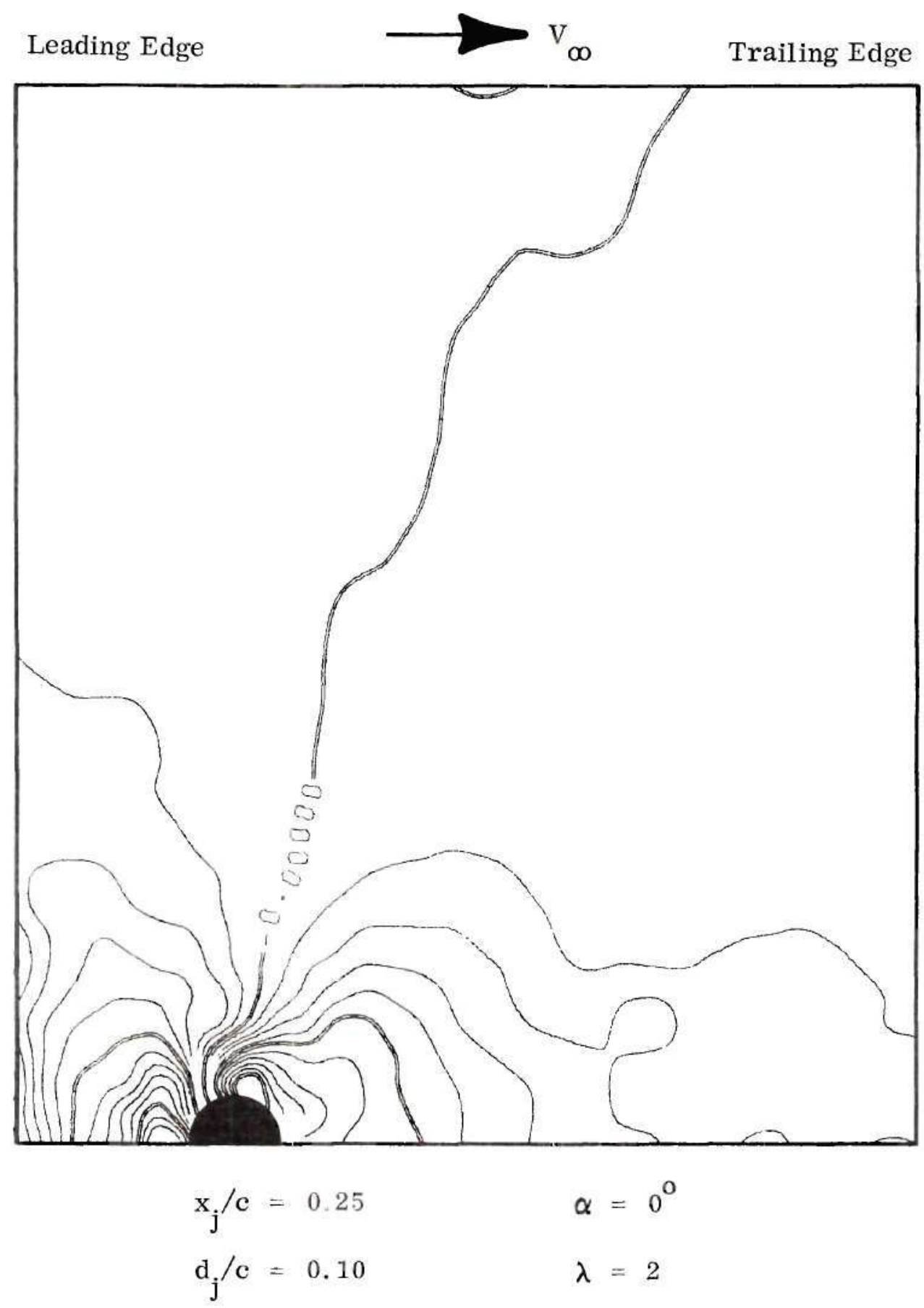

Lower Surface Run No. 53

Figure C-1a. Interference Surface Pressure Distribution. 

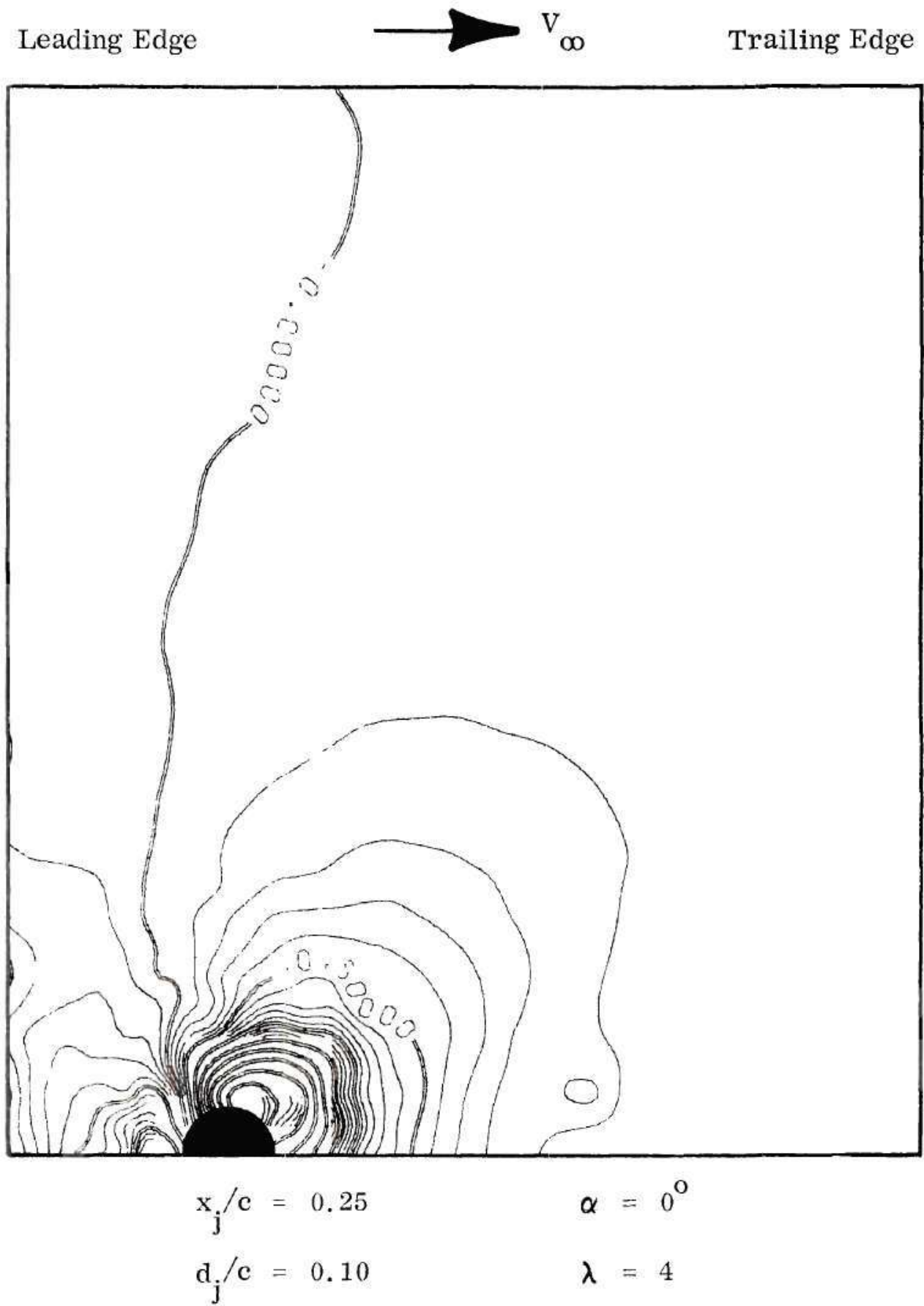

Lower Surface Run No. 42

Figure C-1b. Interference Surface Pressure Distribution. 


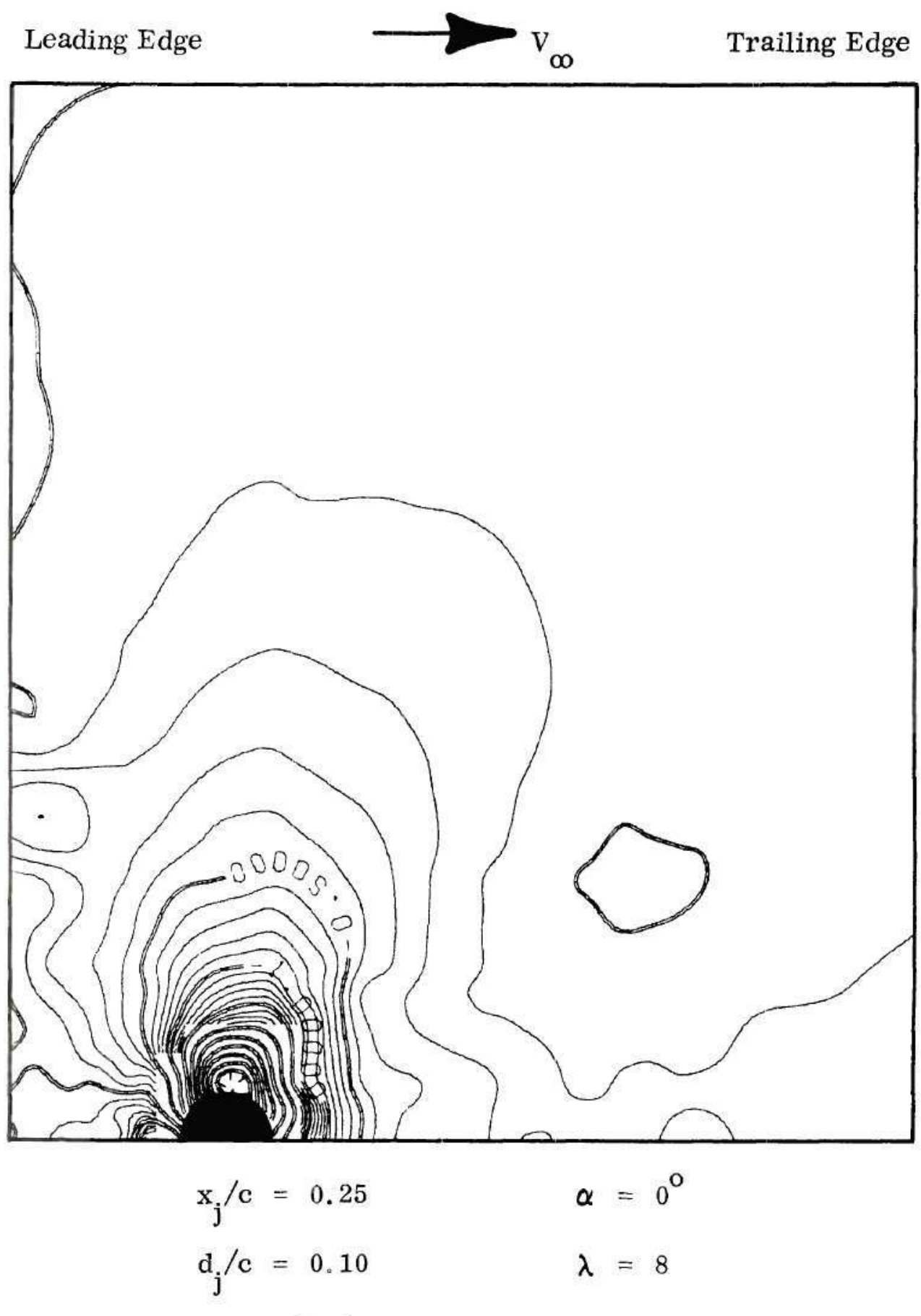

Lower Surface $\quad$ Run No. 41

Figure C-1c. Interference Surface Pressure Distribution. 

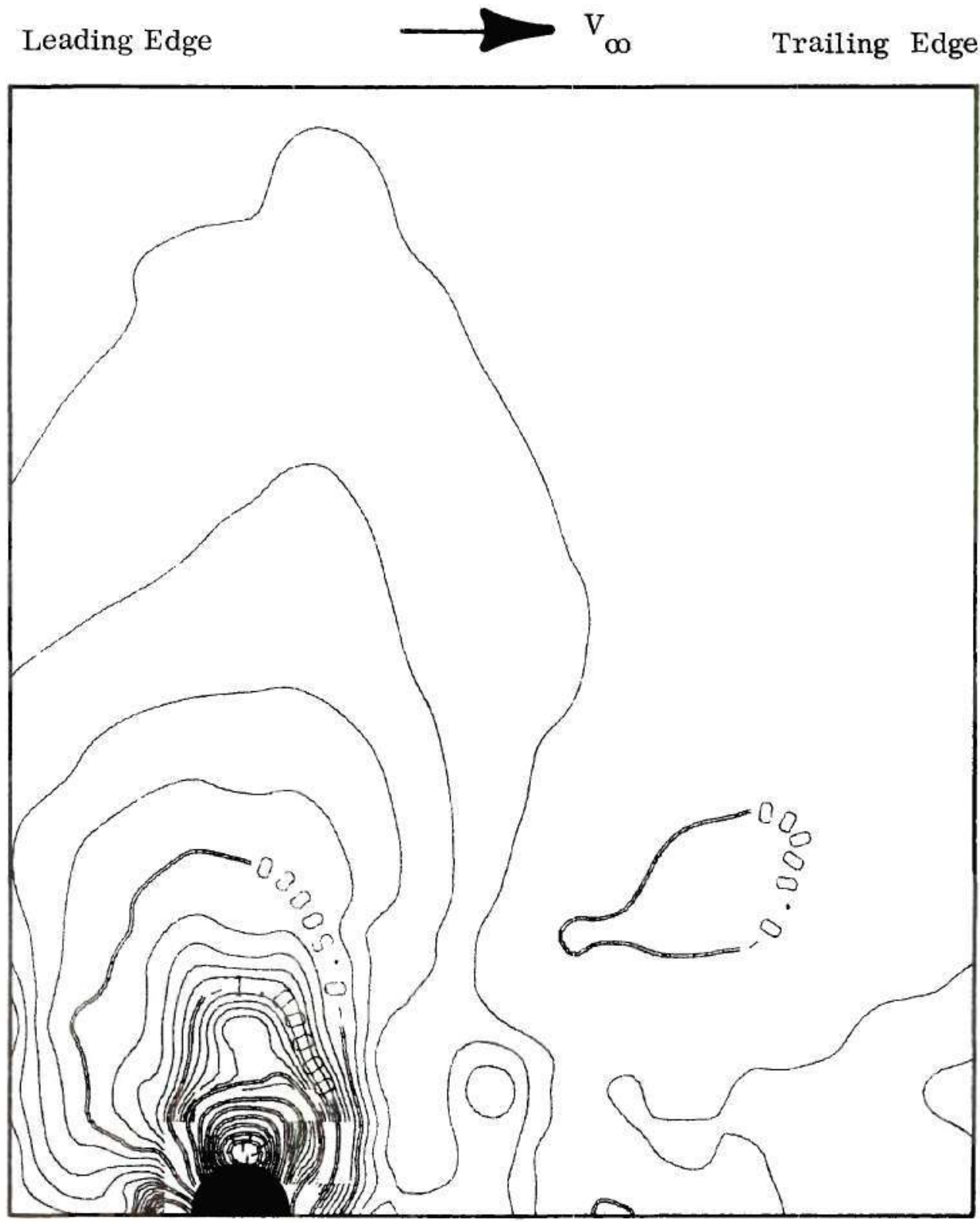

$$
\begin{array}{ll}
\mathrm{x}_{\mathrm{j}} / \mathrm{c}=0.25 & \alpha=0^{\circ} \\
\mathrm{d}_{\mathrm{j}} / \mathrm{c}=0.10 & \lambda=12
\end{array}
$$

Lower Surface

Run No. 52

Figure C-1d. Interference Surface Pressure Distribution. 


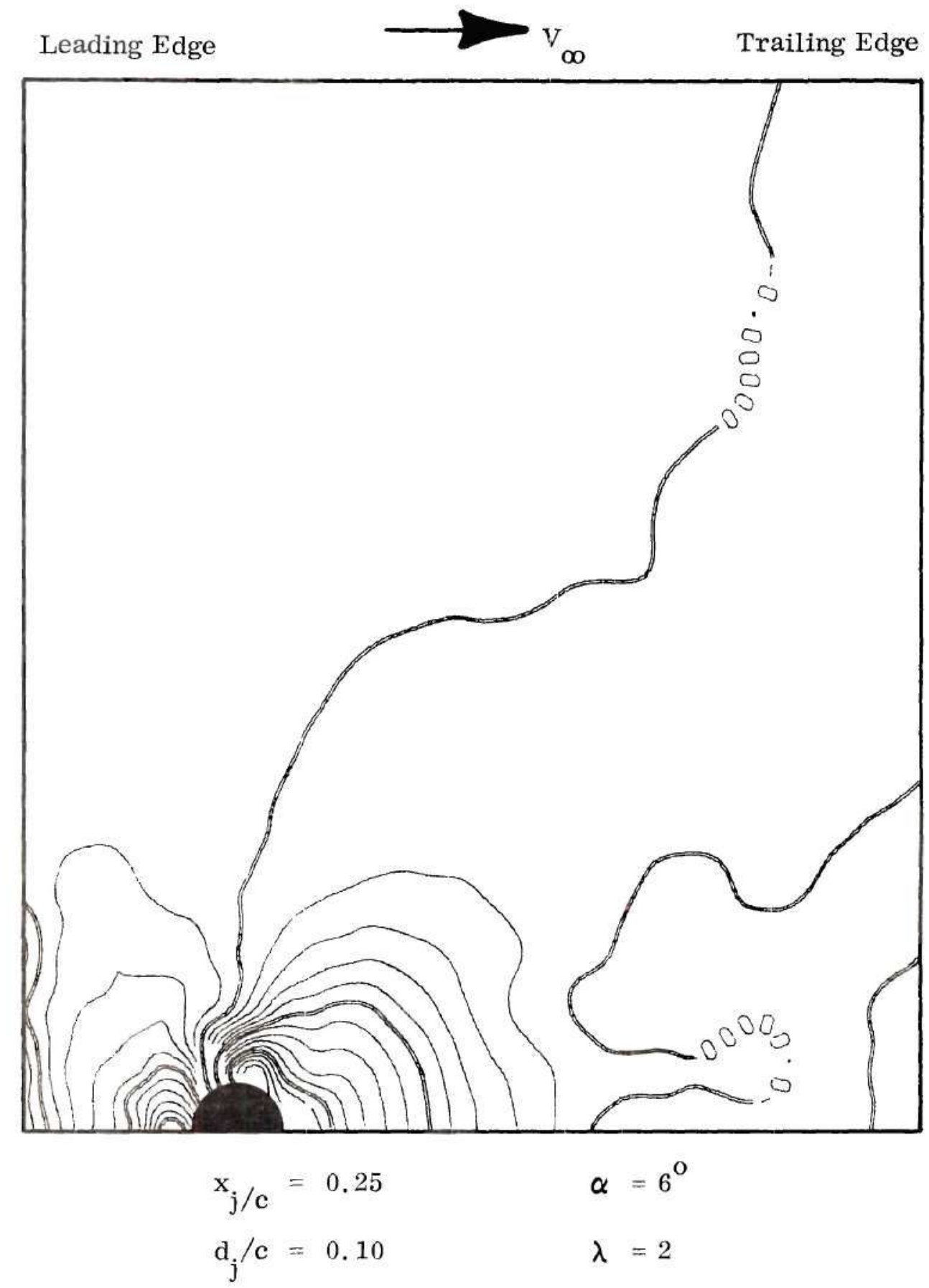

Lower Surface $\quad$ Run No. 46

Figure C-1e. Interference Surface Pressure Distribution. 


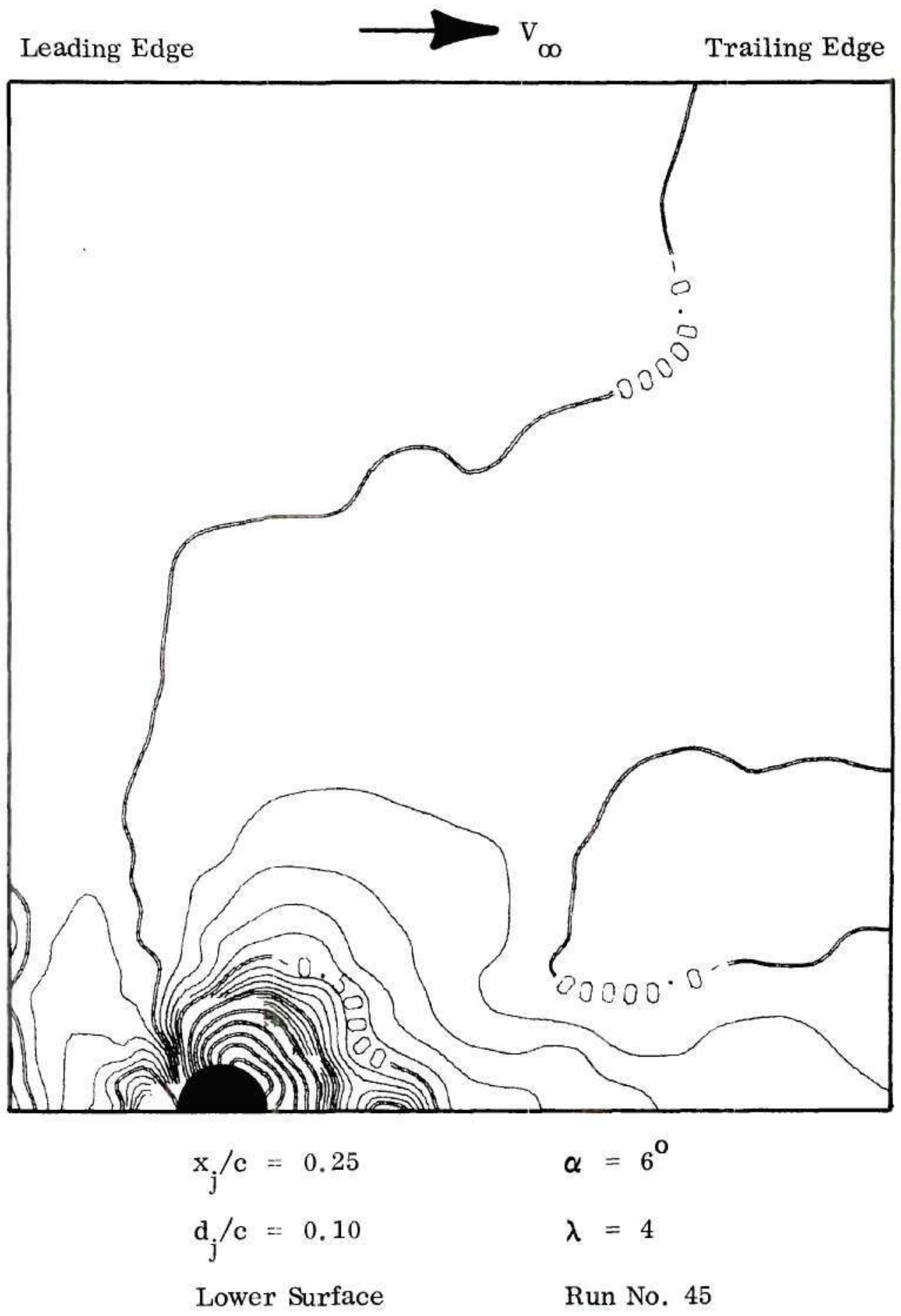

Figure C-1f. Interference Surface Pressure Distribution. 


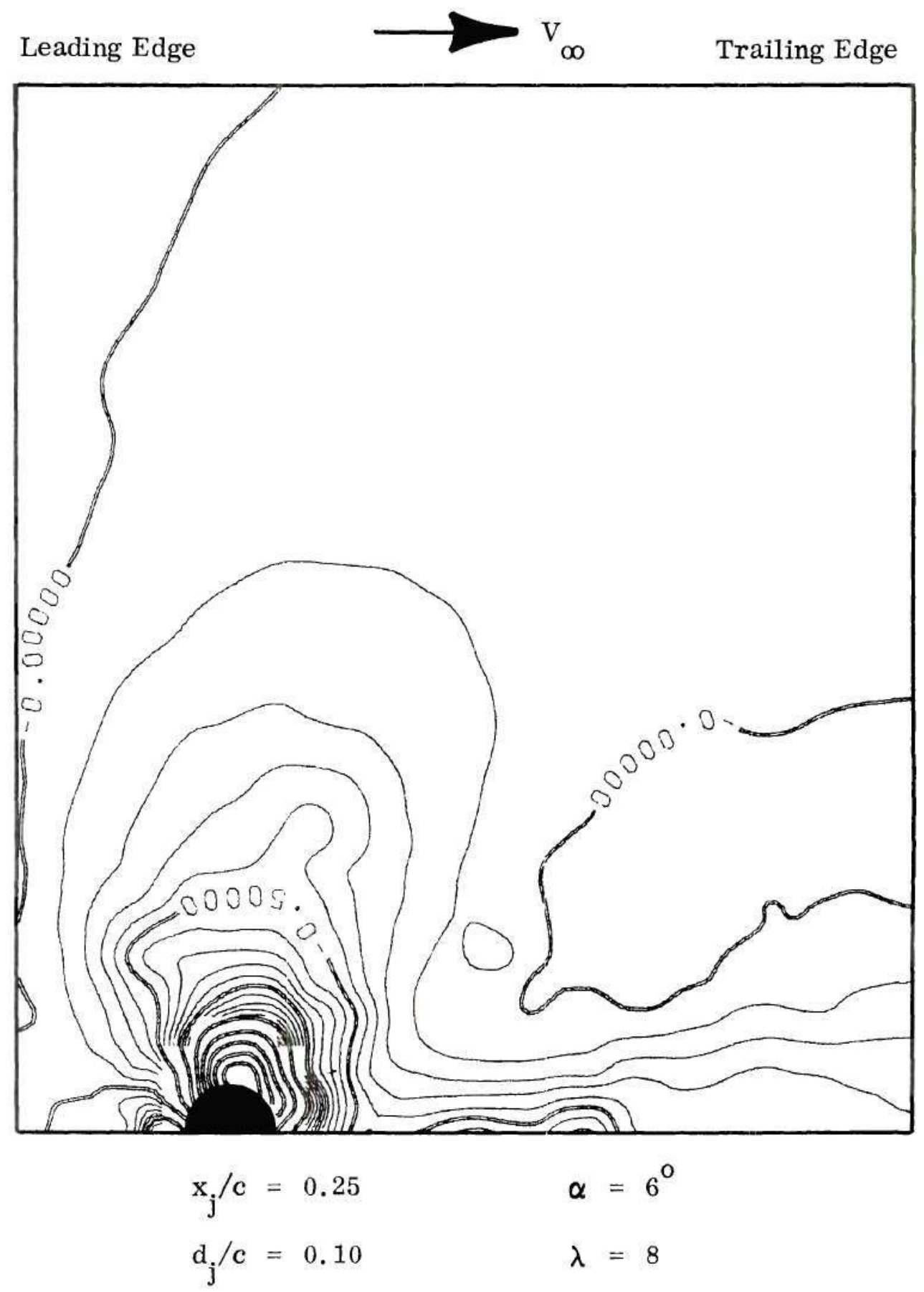

Lower Surface $\quad$ Run No. 44

Figure C-1g. Interference Surface Pressure Distribution. 

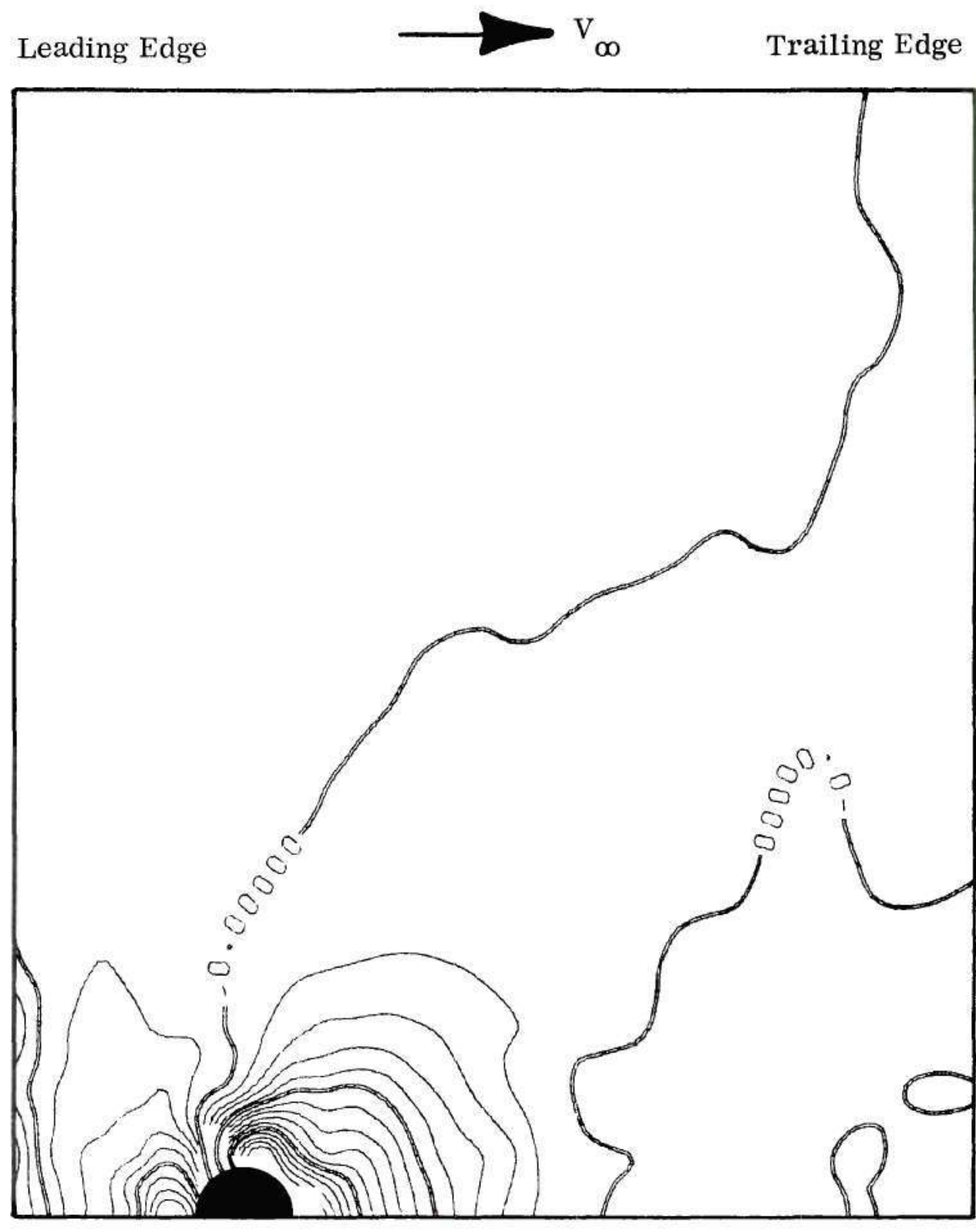

$$
\begin{array}{ll}
\mathrm{x}_{\mathrm{j}} / \mathrm{c}=0.25 & \alpha=9^{\circ} \\
\mathrm{d}_{\mathrm{j}} / \mathrm{c}=0.10 & \lambda=2
\end{array}
$$

Lower Surface Run No. 47

Figure C-1h, Interference Surface Pressure Distribution. 


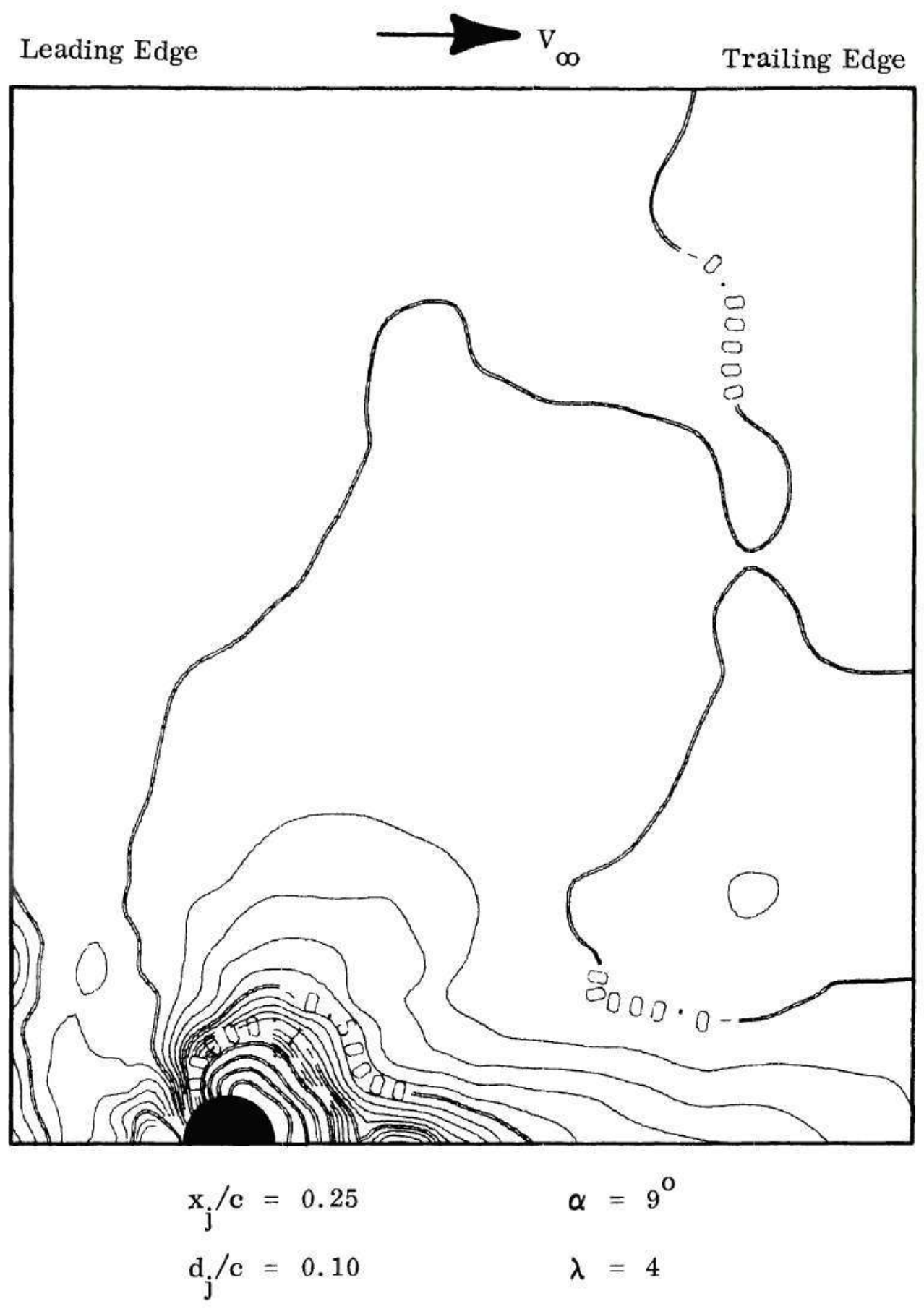

Lower Surface

Run No. 50

Figure C-1i. Interference Surface Pressure Distribution. 

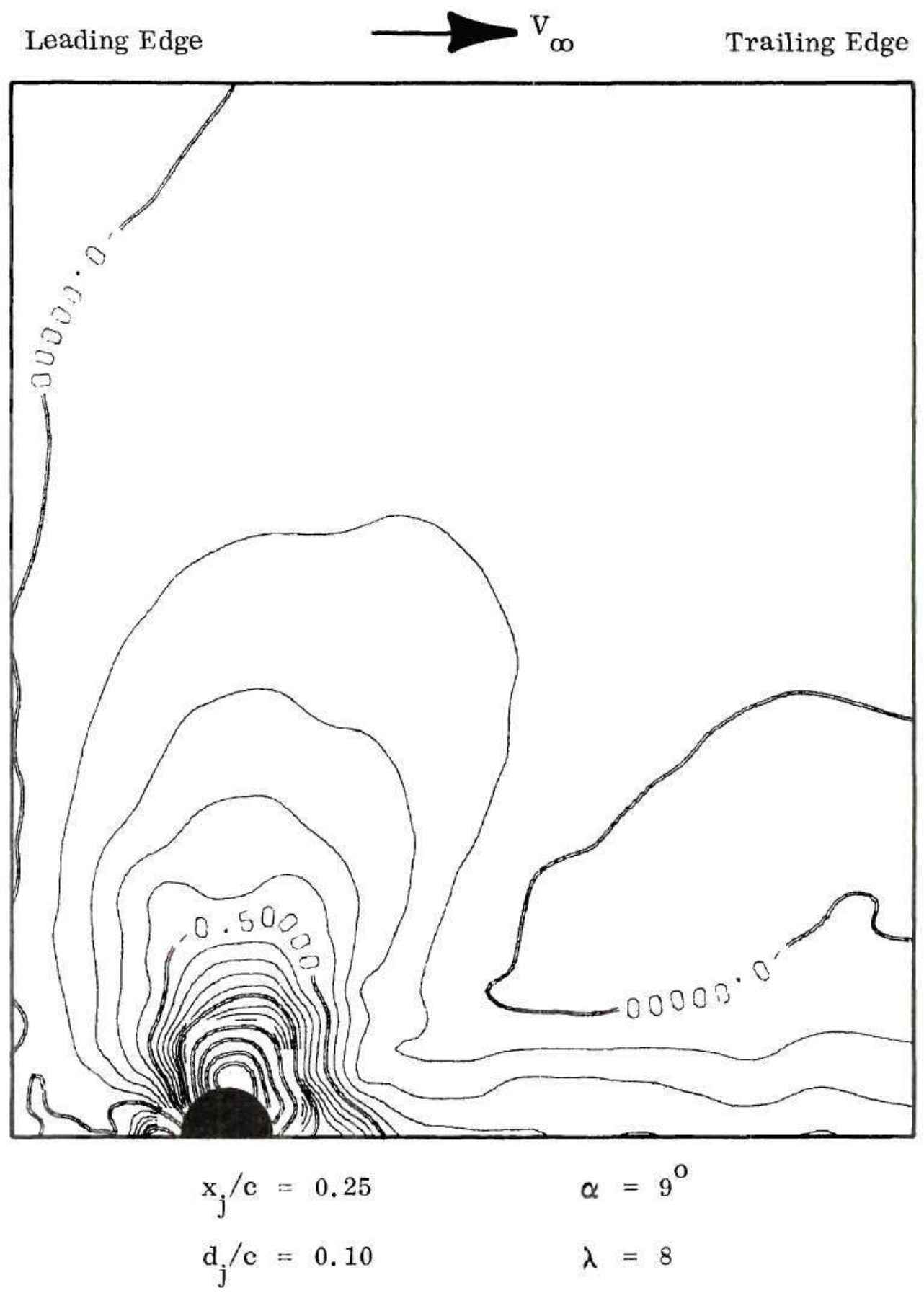

Lower Surface Run No. 49

Figure C-1j. Interference Surface Pressure Distribution. 


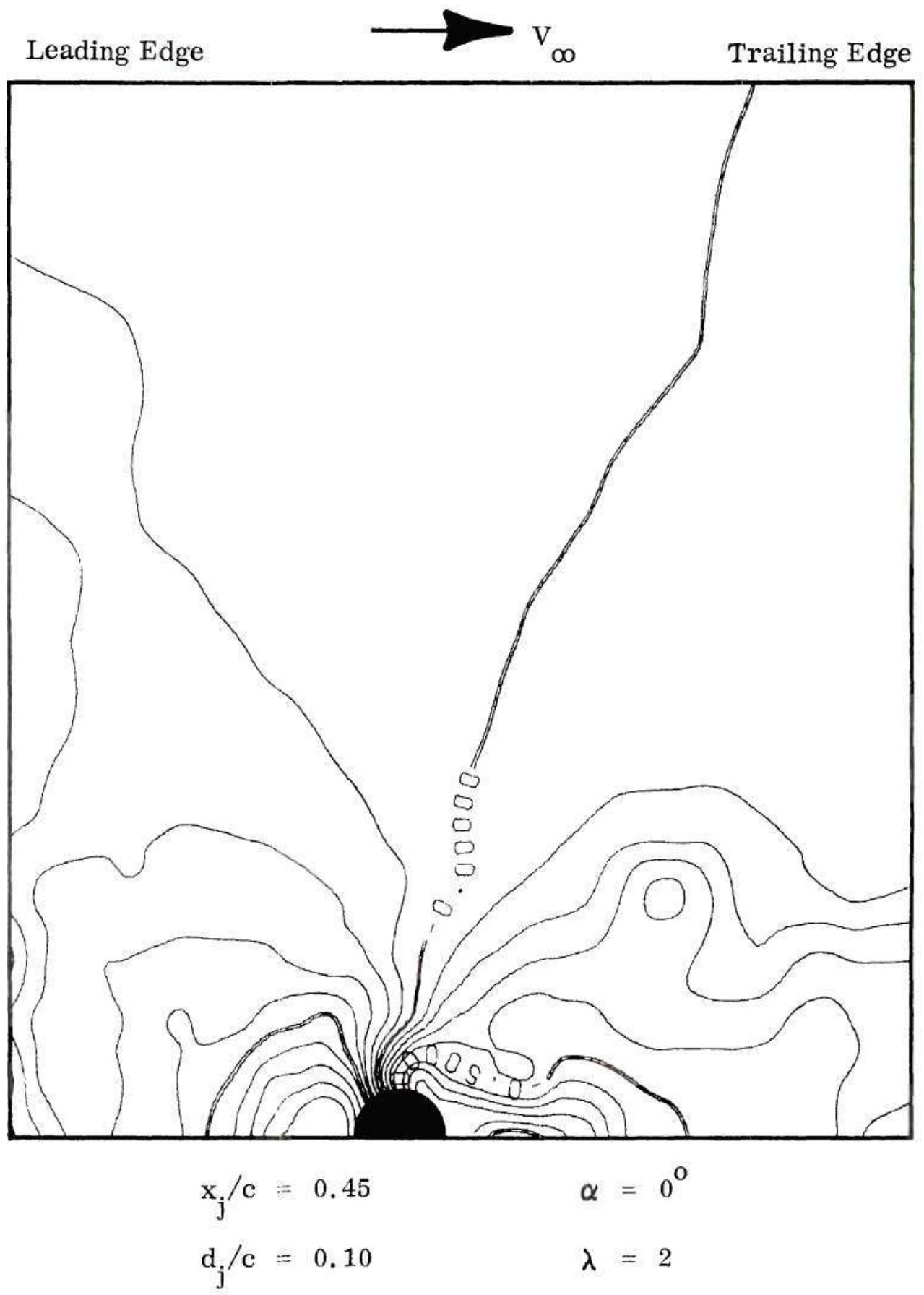

Lower Surface Run No. 30

Figure C-2a. Interference Surface Pressure Distribution. 


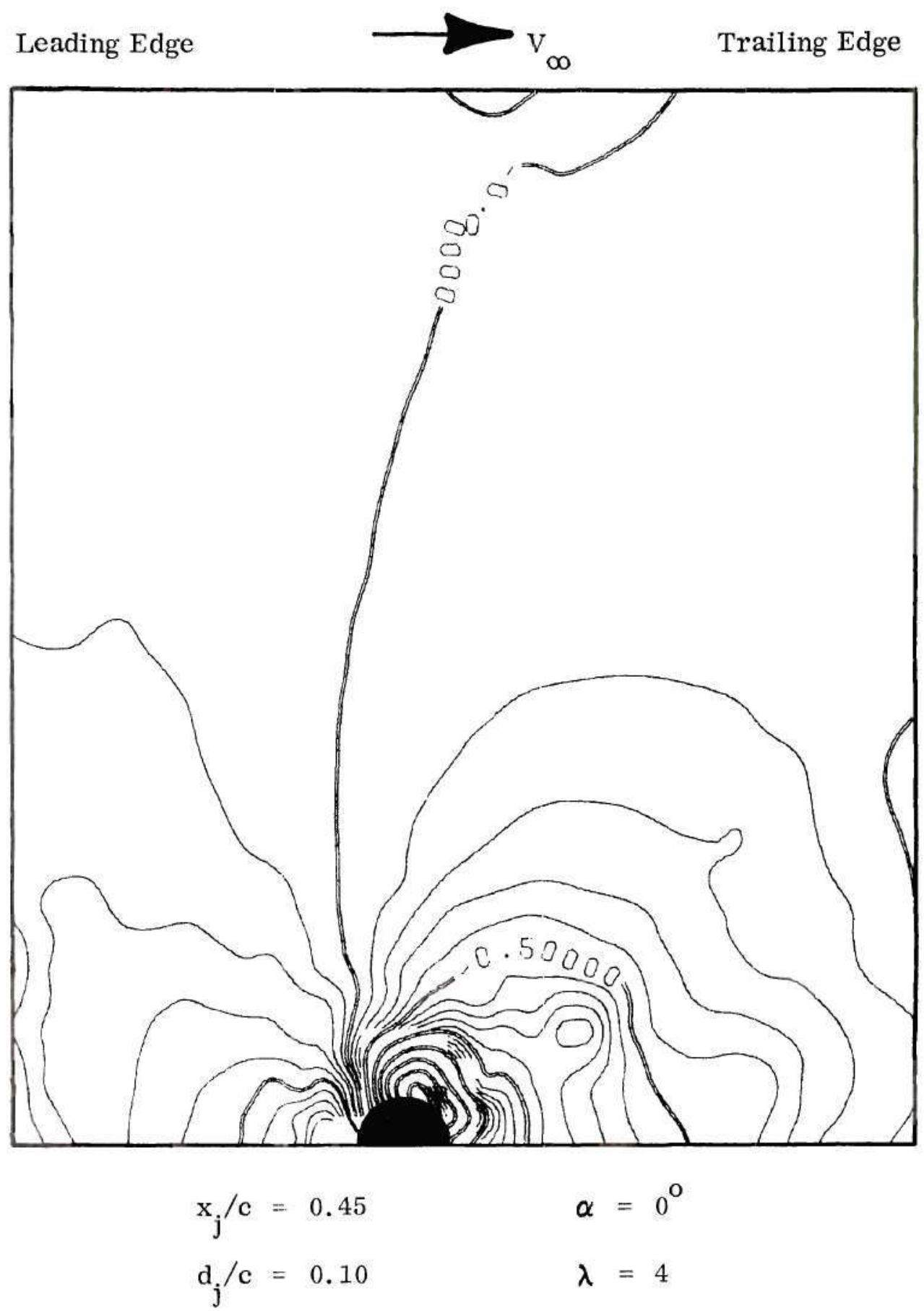

Lower Surface Run No. 29

Figure C-2b. Interference Surface Pressure Distribution. 

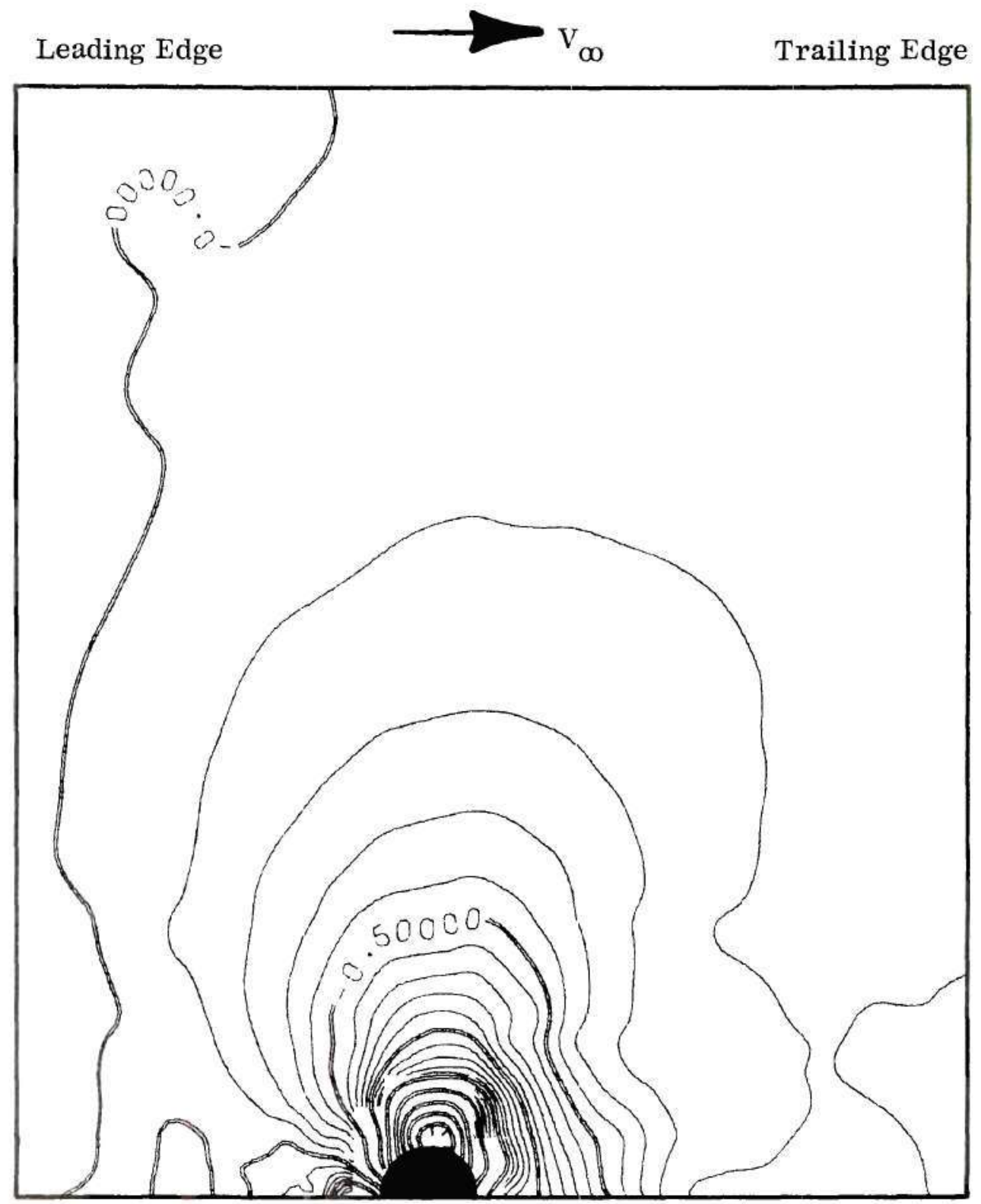
$\mathrm{x}_{\mathrm{j}} / \mathrm{c}=0.45$
$\alpha=0^{\circ}$
$d_{j} / c=0.10$
$\lambda=8$

Lower Surface

Run No. 28

Figure C-2c. Interference Surface Pressure Distribution. 

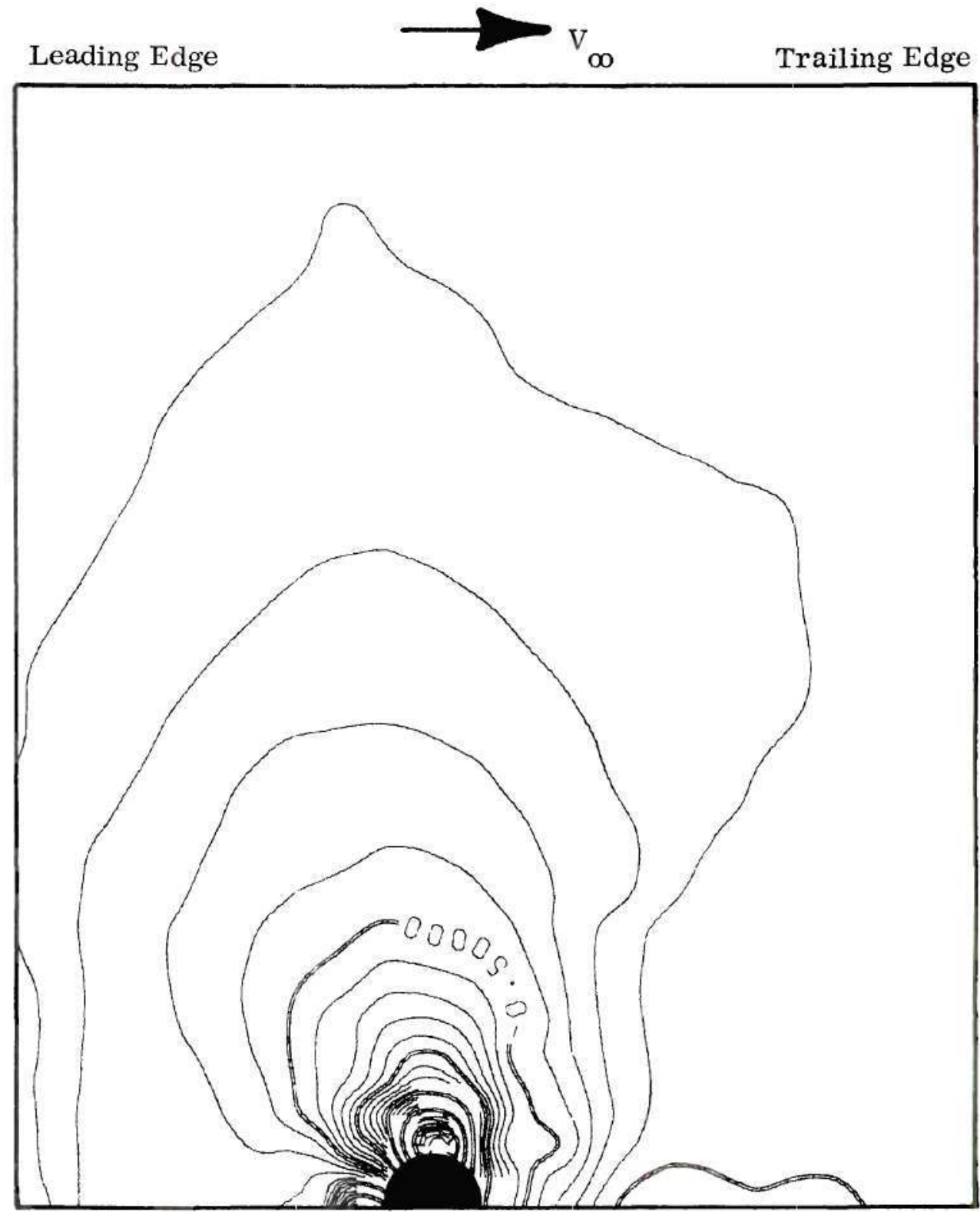
$\mathrm{x}_{\mathrm{j}} / \mathrm{c}=0.45$
$\alpha=0^{0}$
$\mathrm{d}_{\mathrm{j}} / \mathrm{c}=0.10$
$\lambda=12$

Lower Surface

Run No. 38

Figure C-2d. Interference Surface Pressure Distribution. 


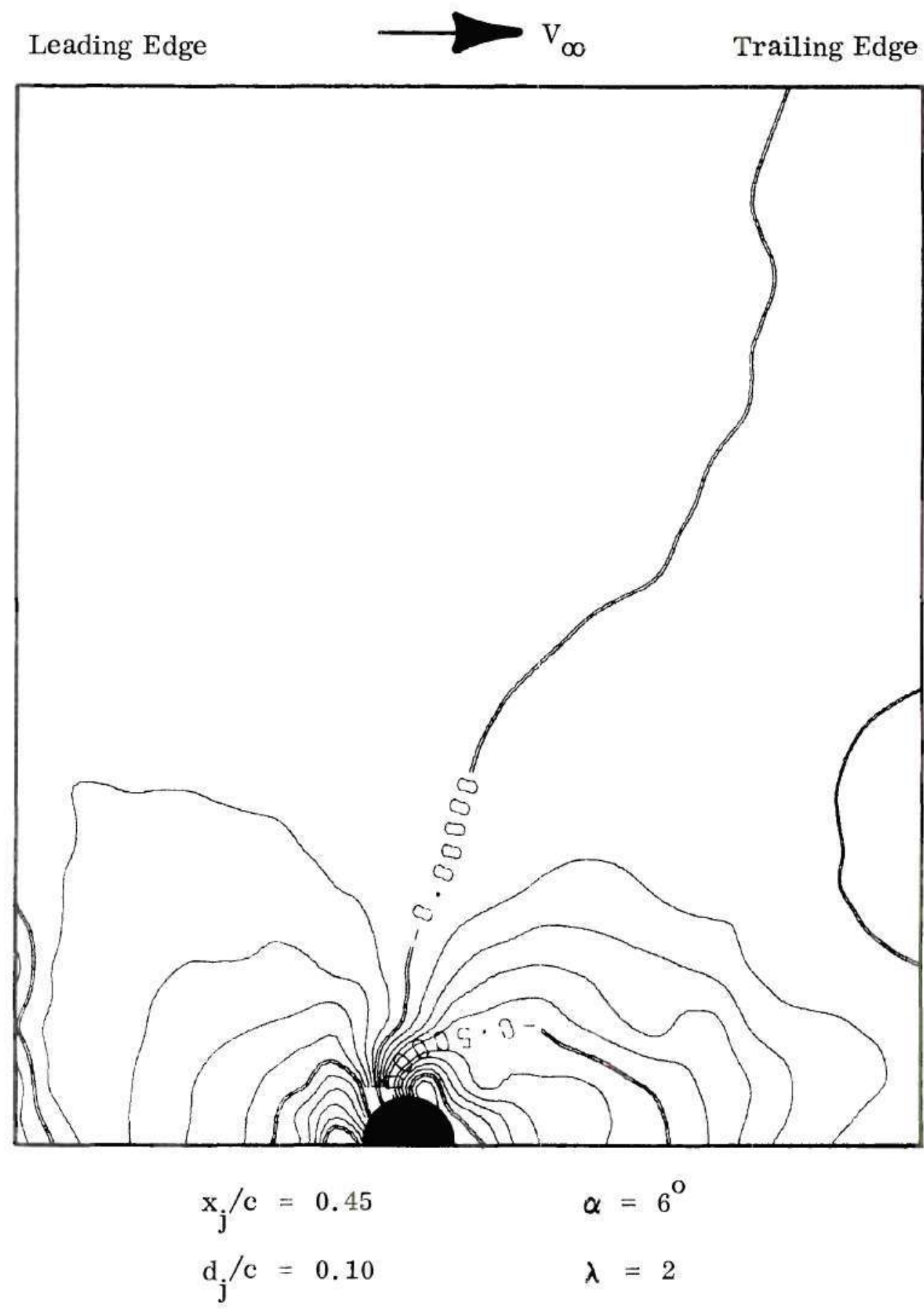

Lower Surface Run No. 33

Figure C-2e. Interference Surface Pressure Distribution. 


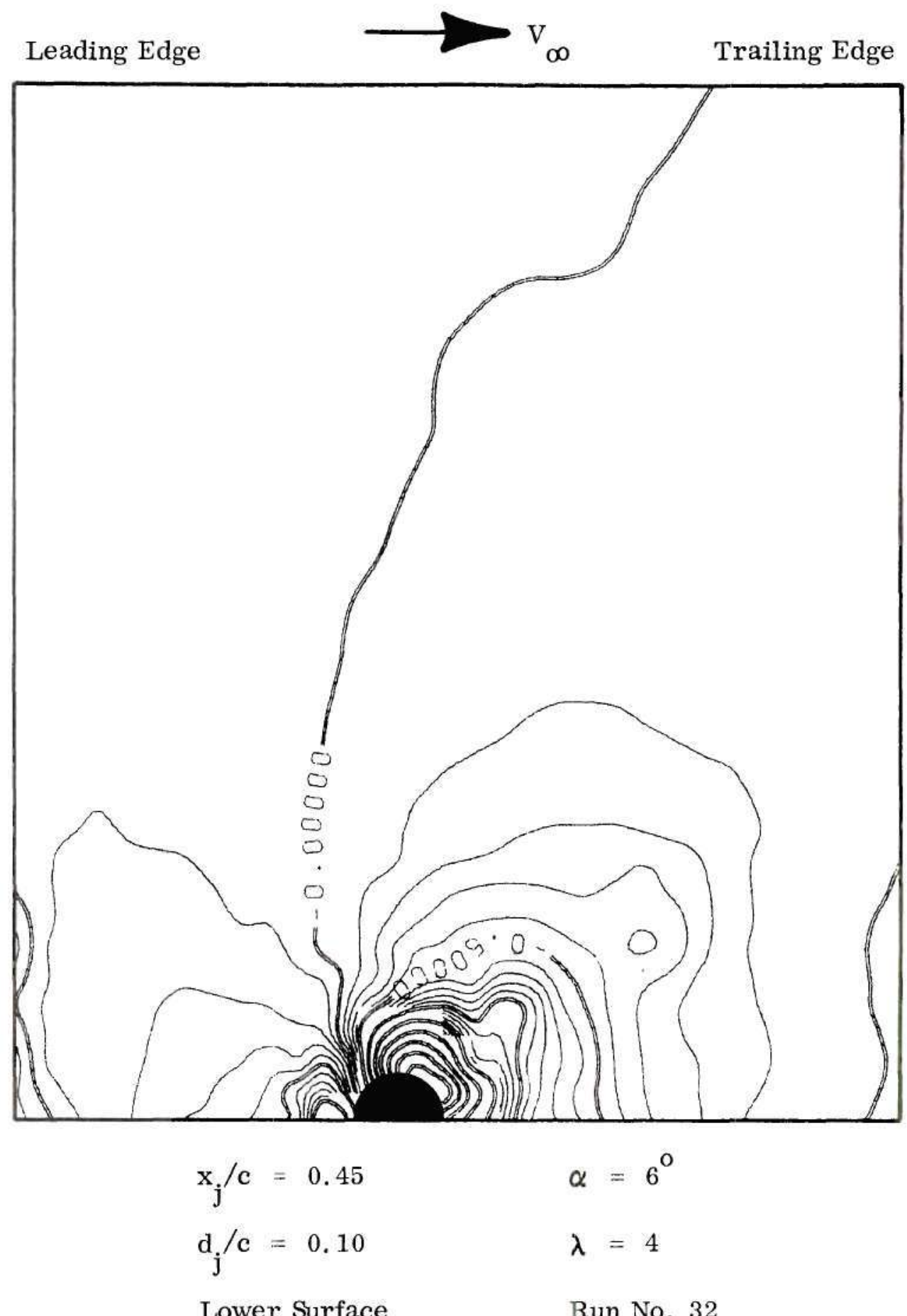

Figure C-2f. Interference Surface Pressure Distribution. 


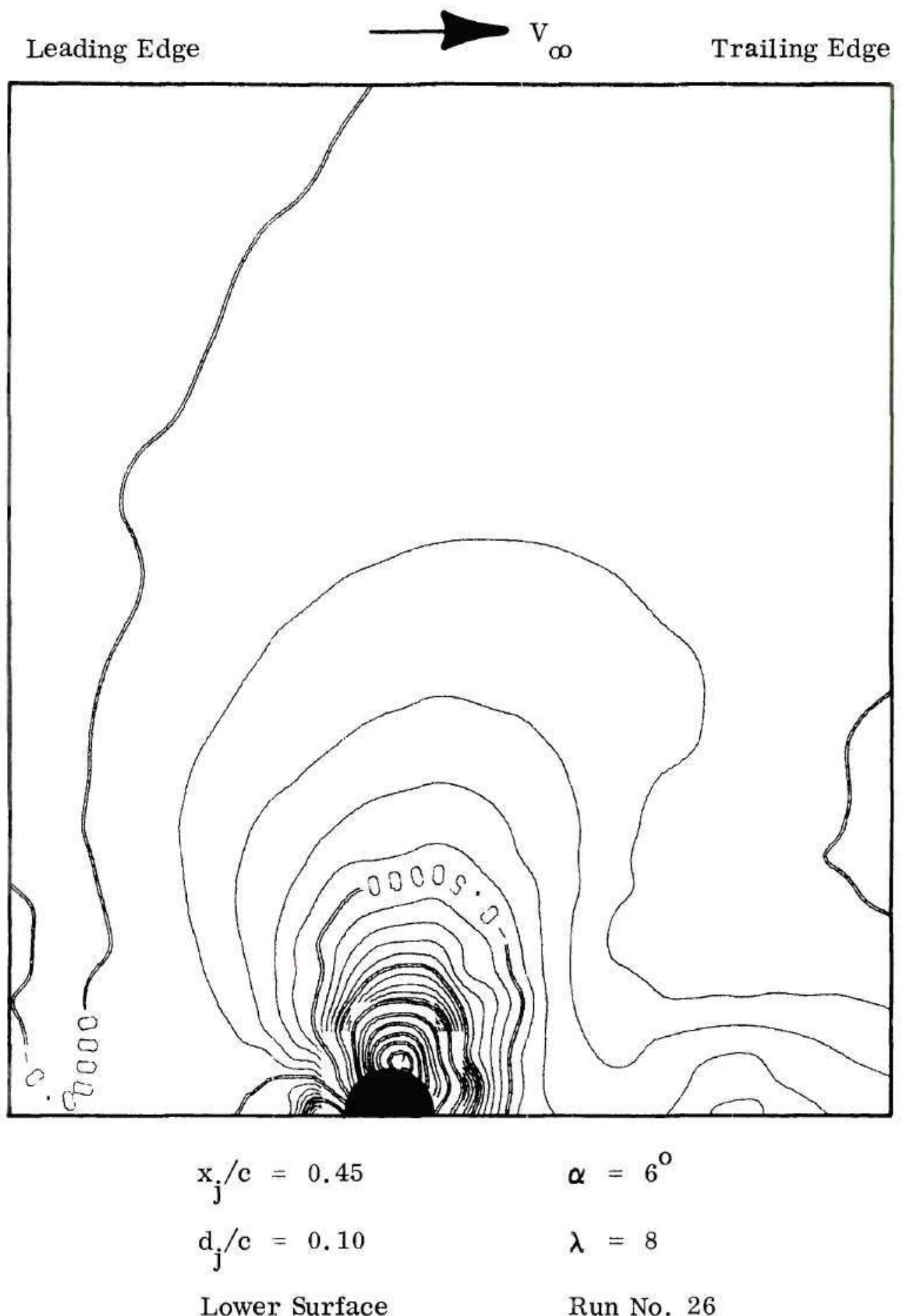

Figure C-2g. Interference Surface Pressure Distribution. 


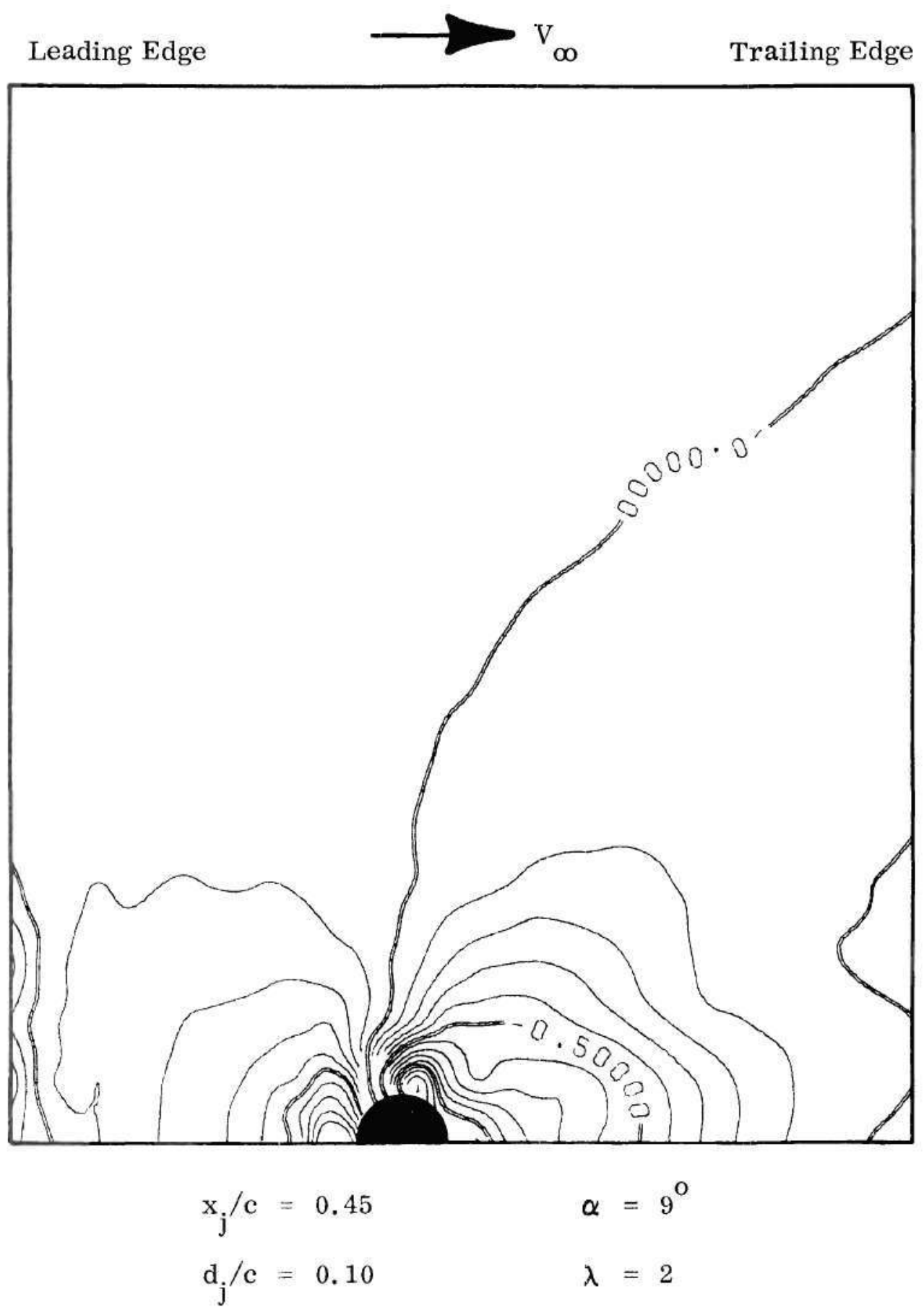

Lower Surface $\quad$ Run No. 37

Figure C-2h. Interference Surface Pressure Distribution. 

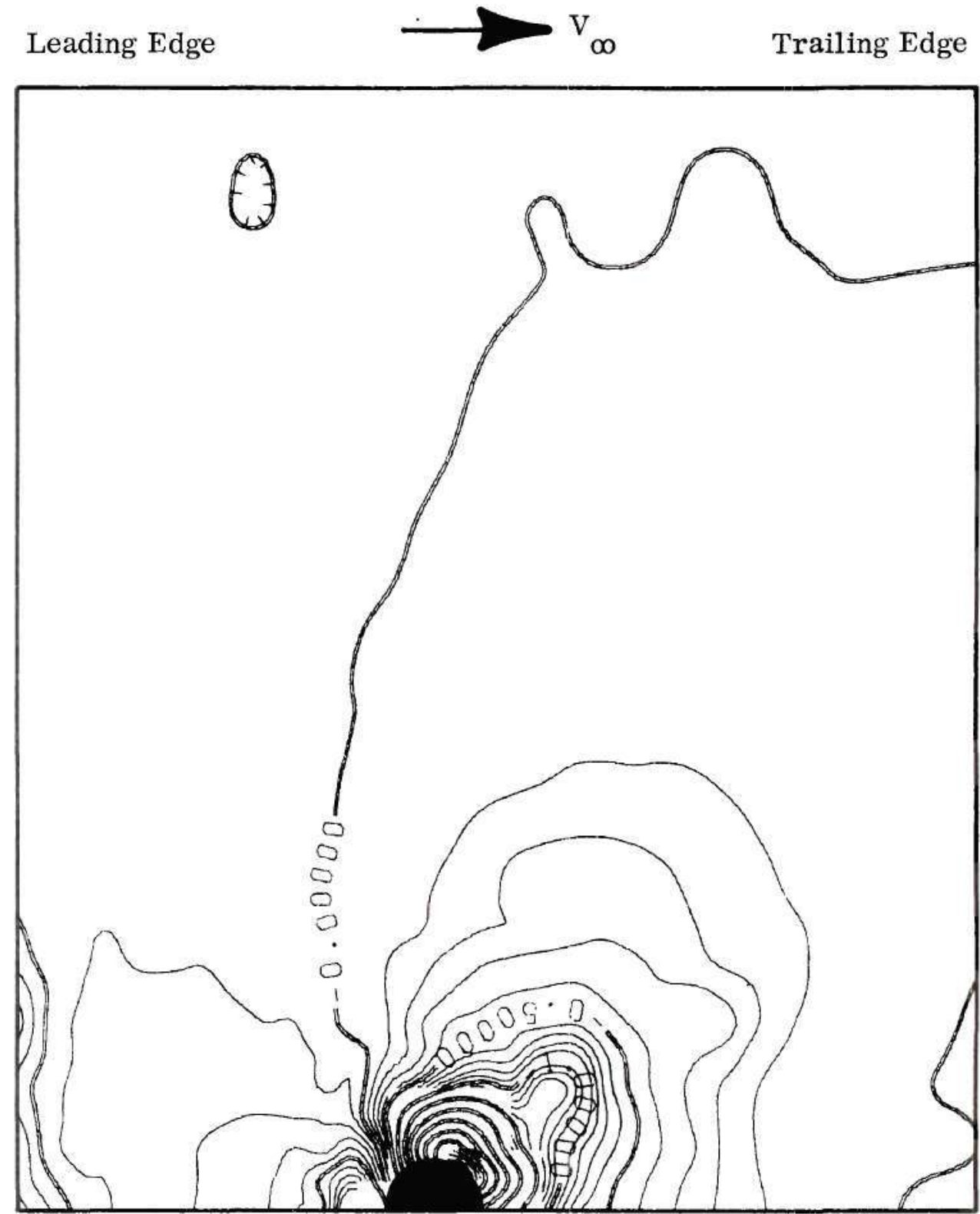

$$
\begin{array}{ll}
\mathrm{x}_{\mathrm{j}} / \mathrm{c}=0.45 & \alpha=9^{\circ} \\
\mathrm{d}_{\mathrm{j}} / \mathrm{c}=0.10 & \lambda=4
\end{array}
$$

Lower Surface

Run No. 36

Figure C-2i. Interference Surface Pressure Distribution. 


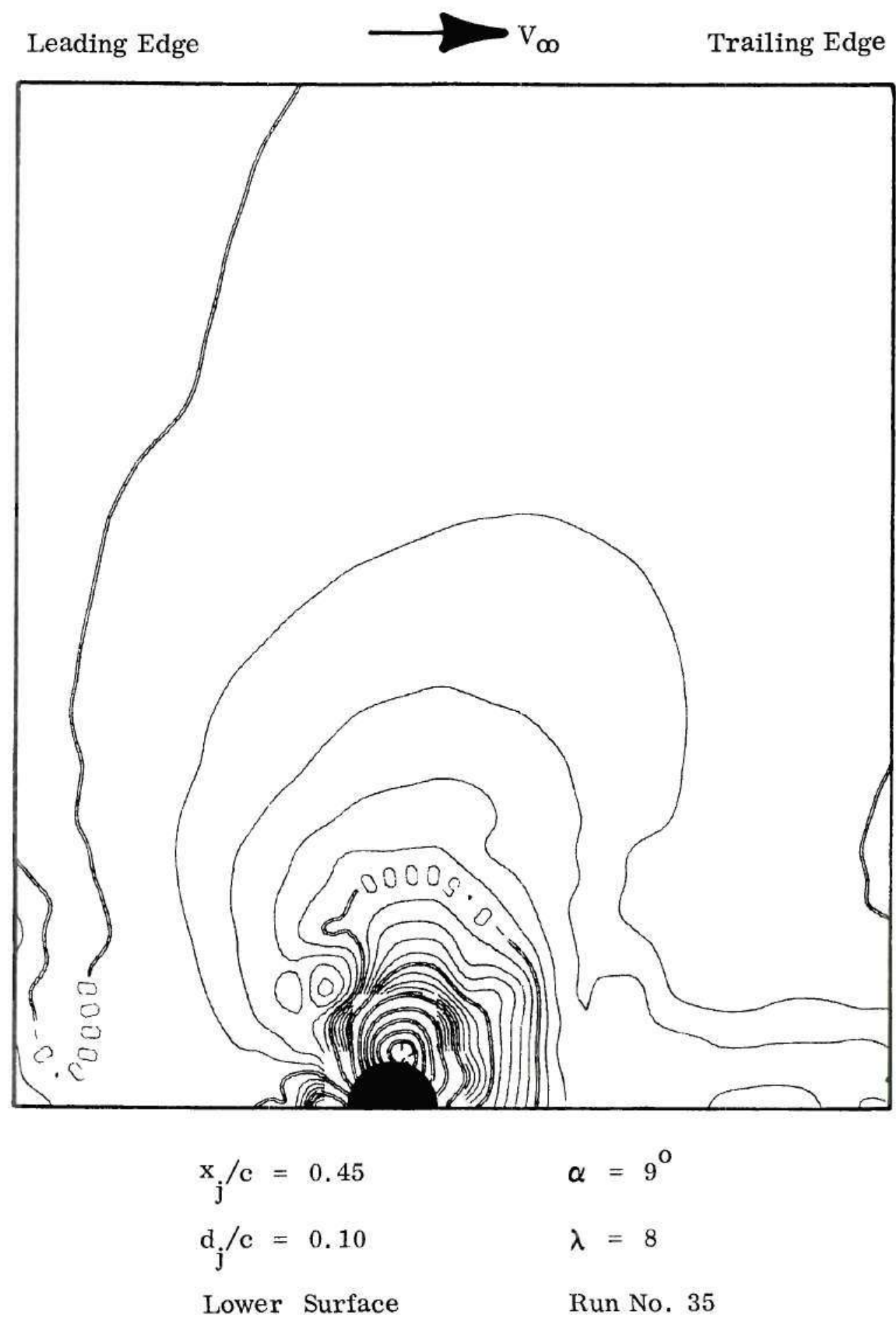

Figure C-2j. Interference Surface Pressure Distribution. 


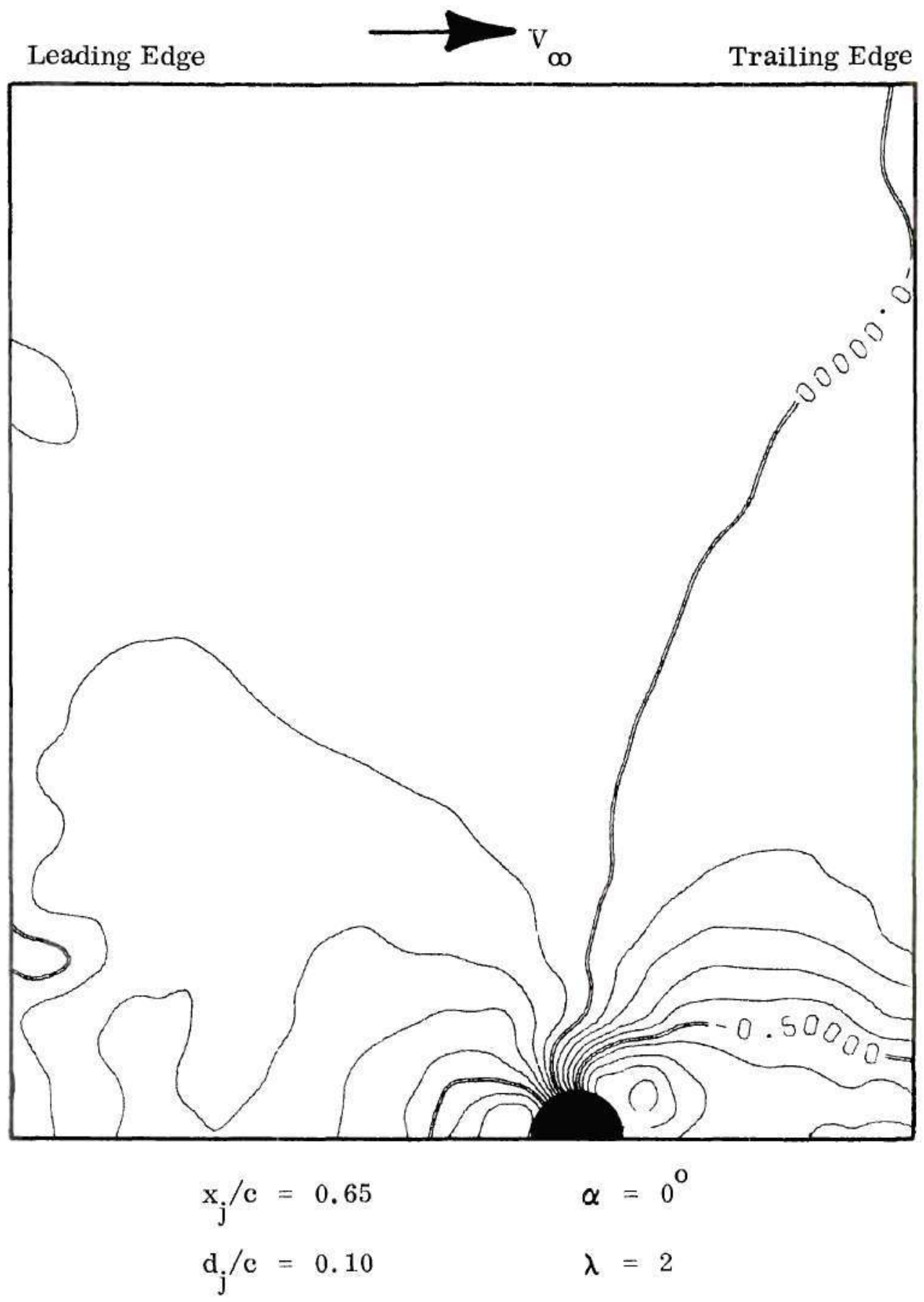

Lower Surface

Run No. 59

Figure C-3a. Interference Surface Pressure Distribution. 


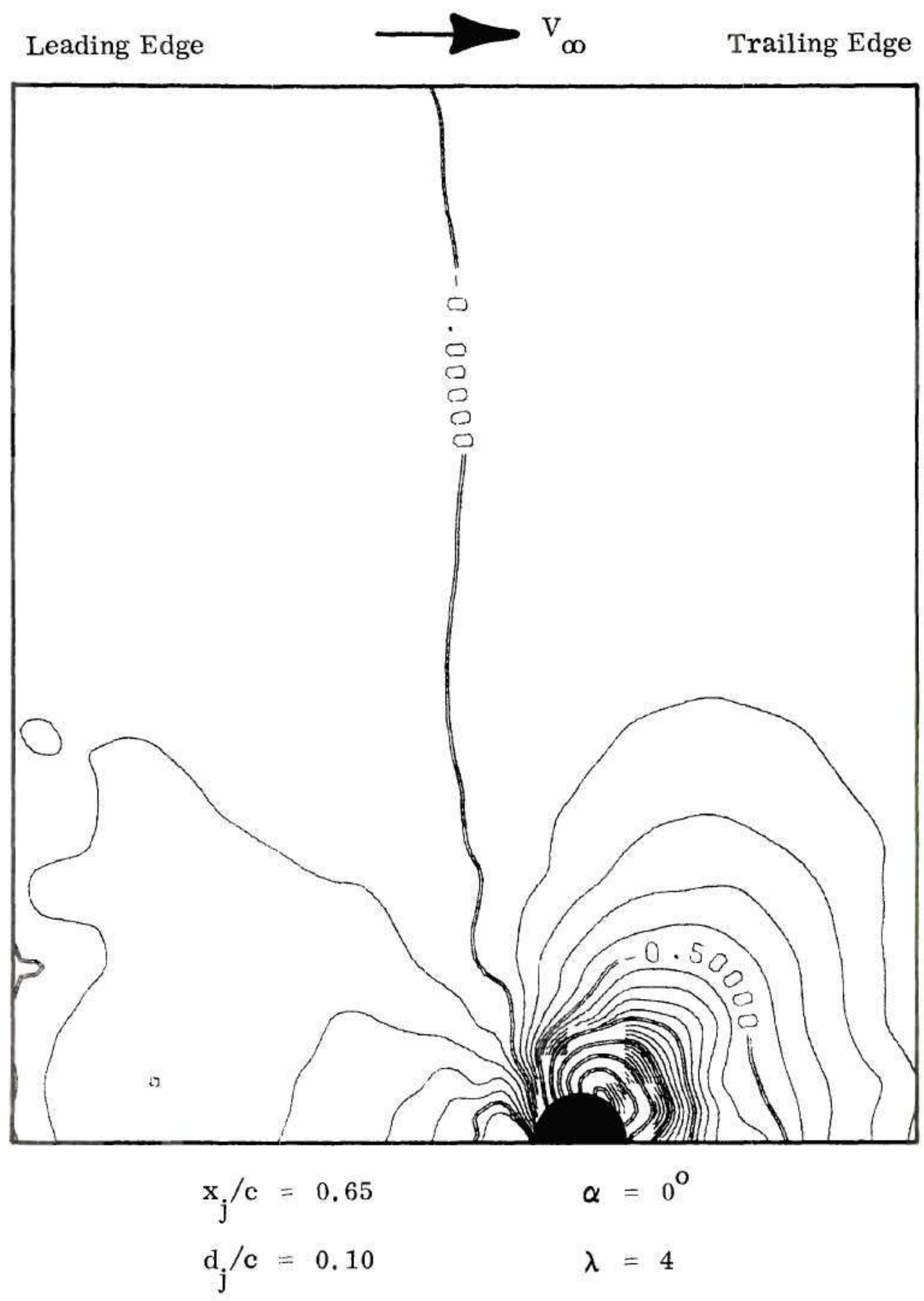

Lower Surface Run No. 57

Figure C-3b. Interference Surface Pressure Distribution. 


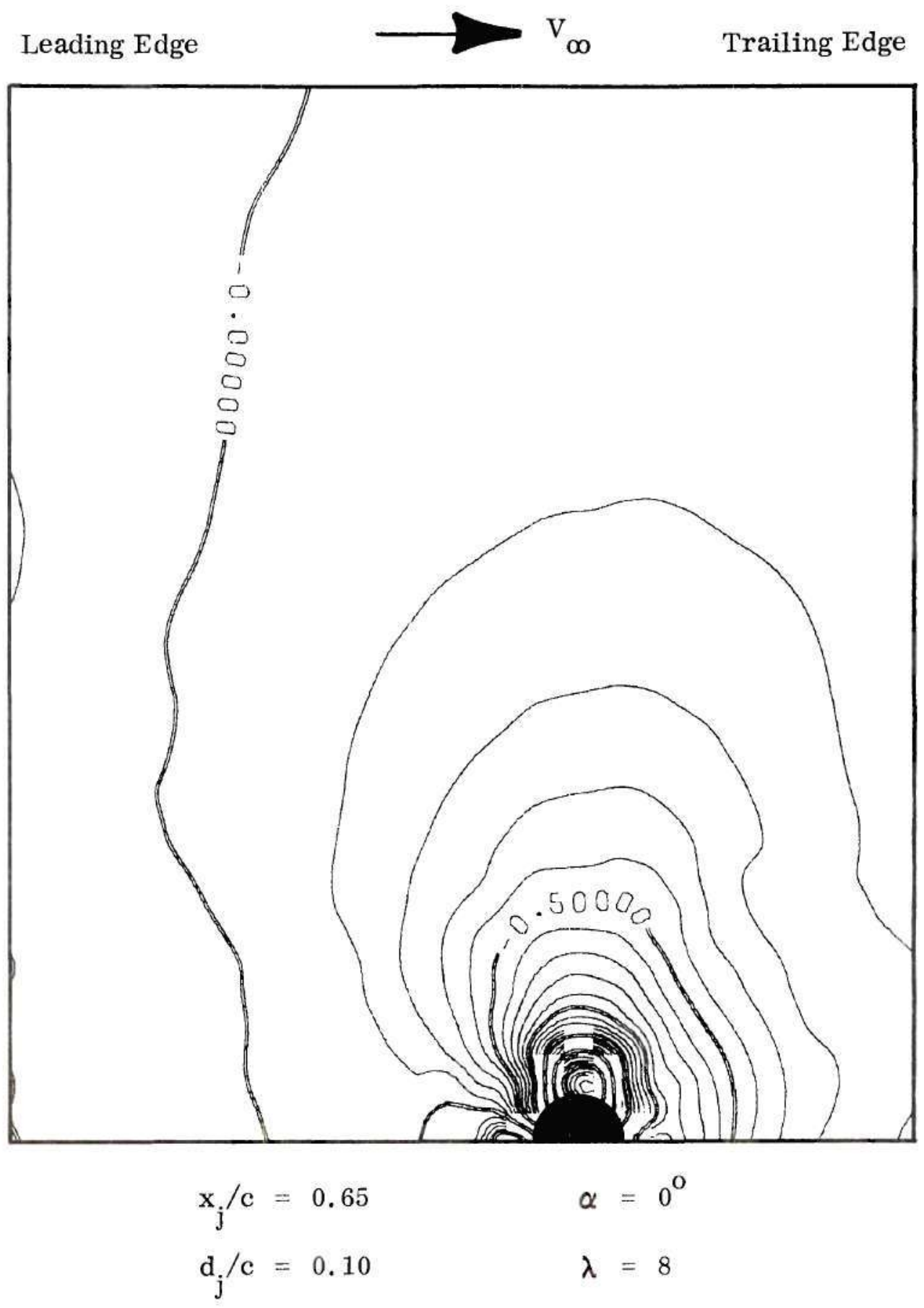

Lower Surface Run No. 55

Figure C-3c. Interference Surface Pressure Distribution. 


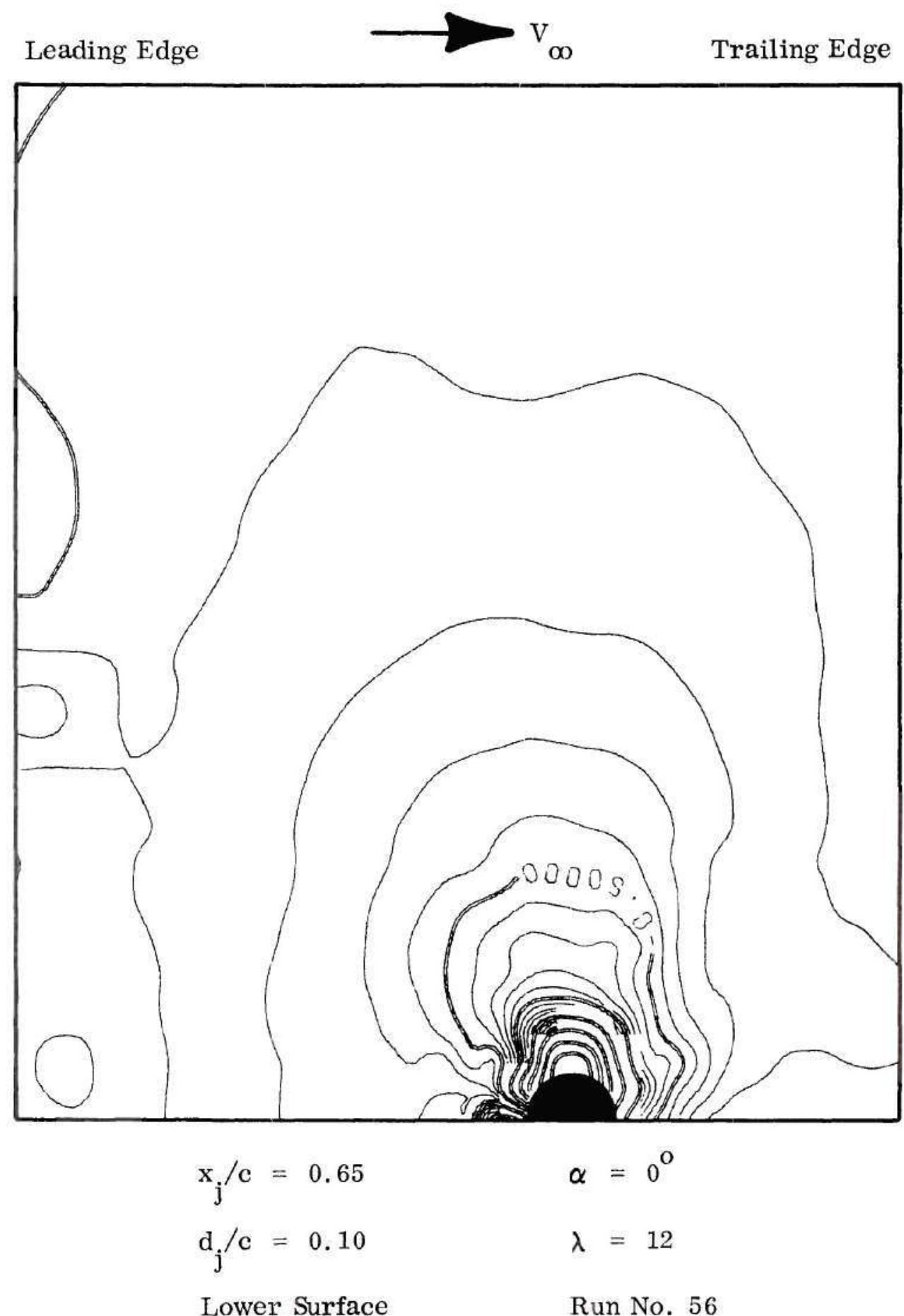

Figure C-3d. Interference Surface Pressure Distribution. 


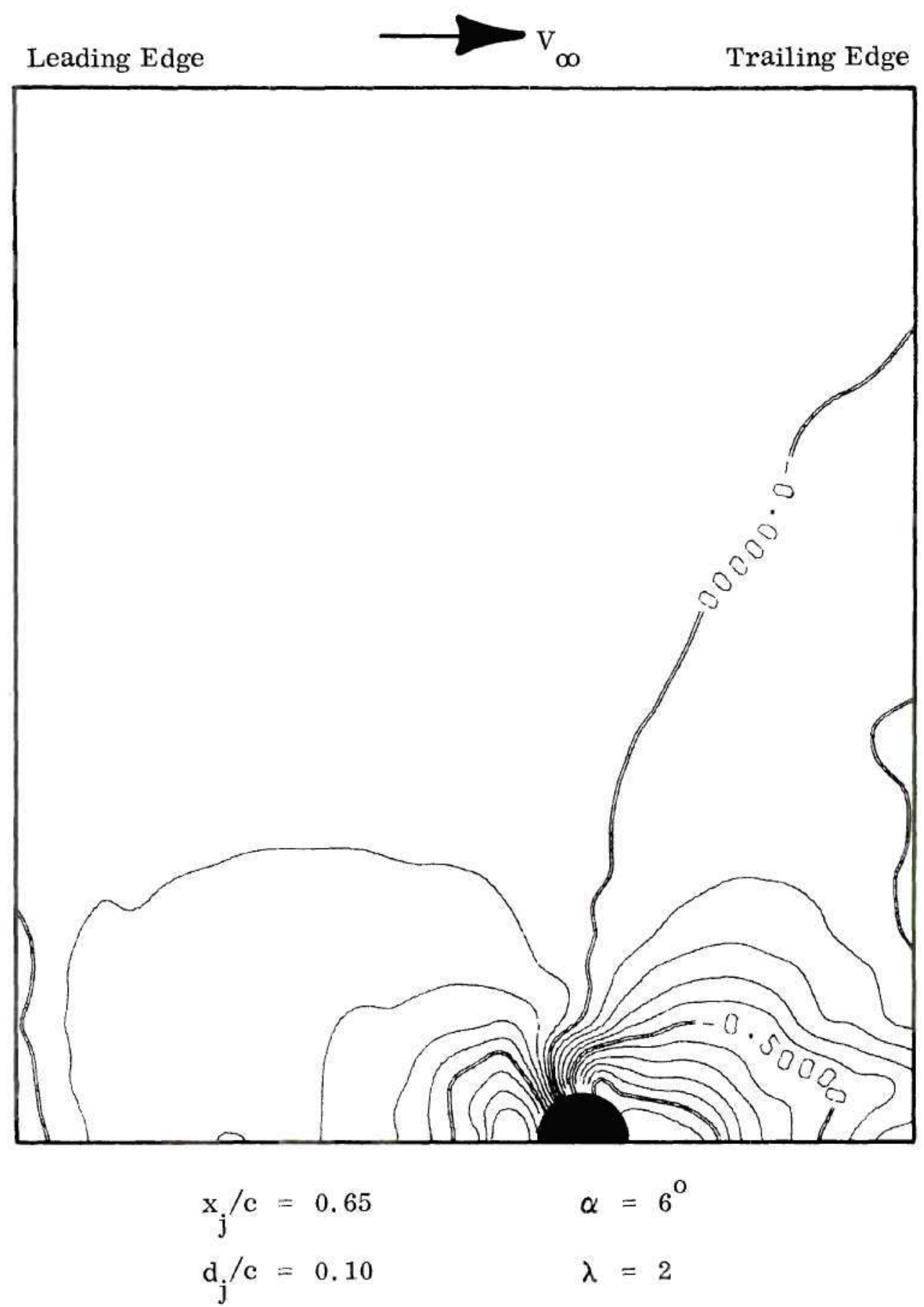

Lower Surface Run No. 63

Figure C-3e. Interference Surface Pressure Distribution. 


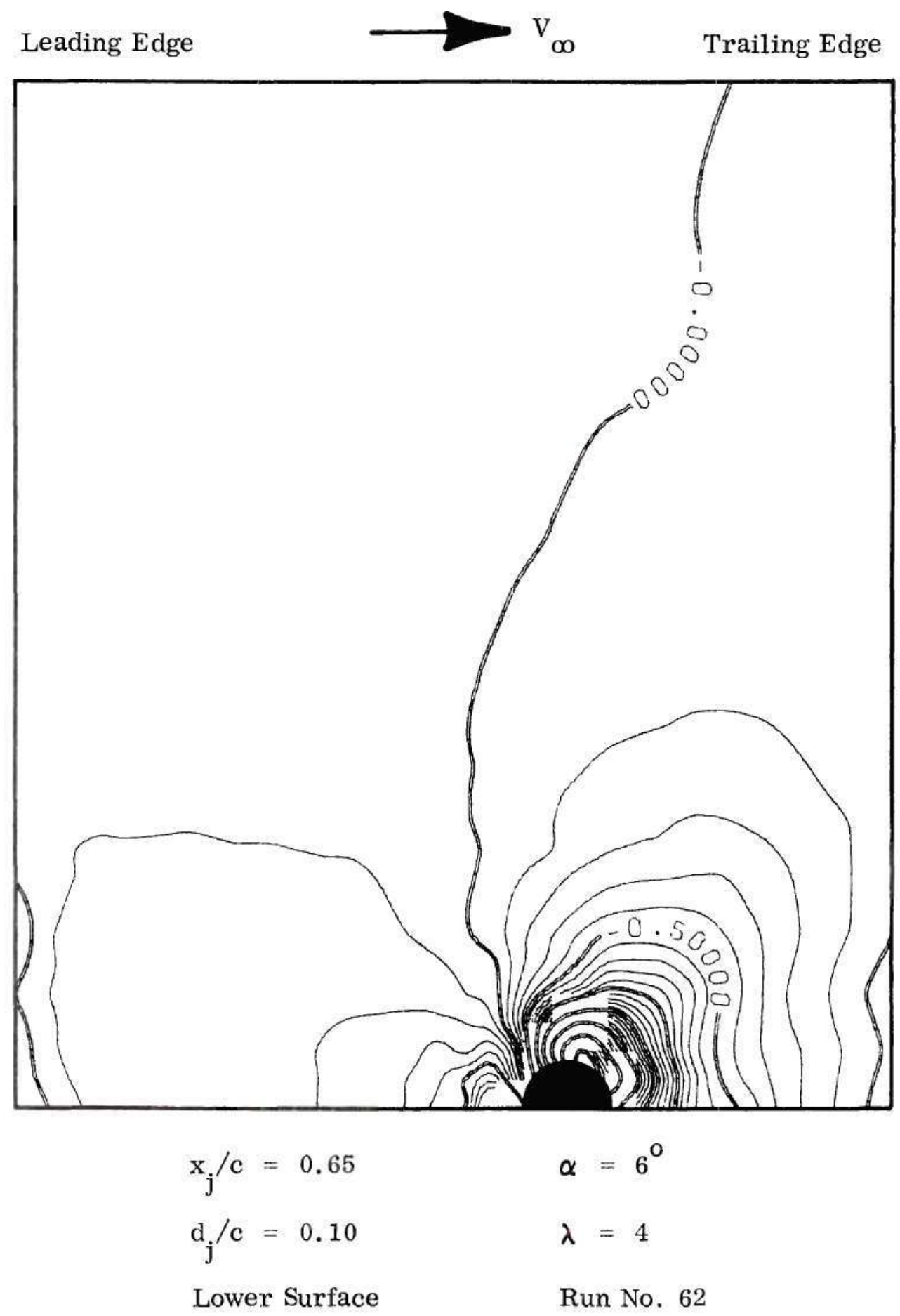

Figure C-3f. Interference Surface Pressure Distribution. 

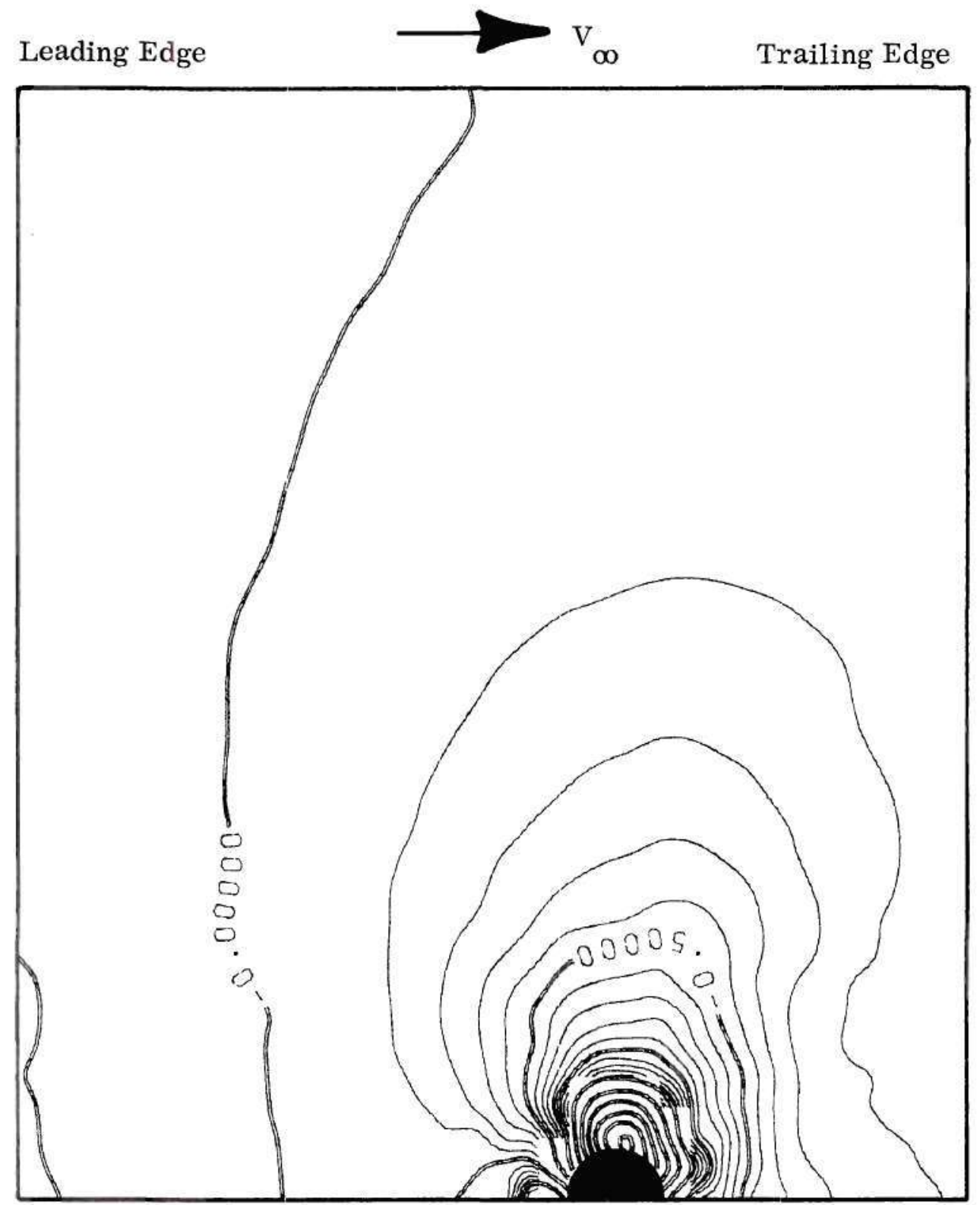
$\mathrm{x}_{\mathrm{j}} / \mathrm{c}=0.65$
$\alpha=6^{\circ}$
$\mathrm{d}_{\mathrm{j}} / \mathrm{c}=0.10$
$\lambda=8$

Lower Surface

Run No. 69

Figure C-3g. Interference Surface Pressure Distribution. 


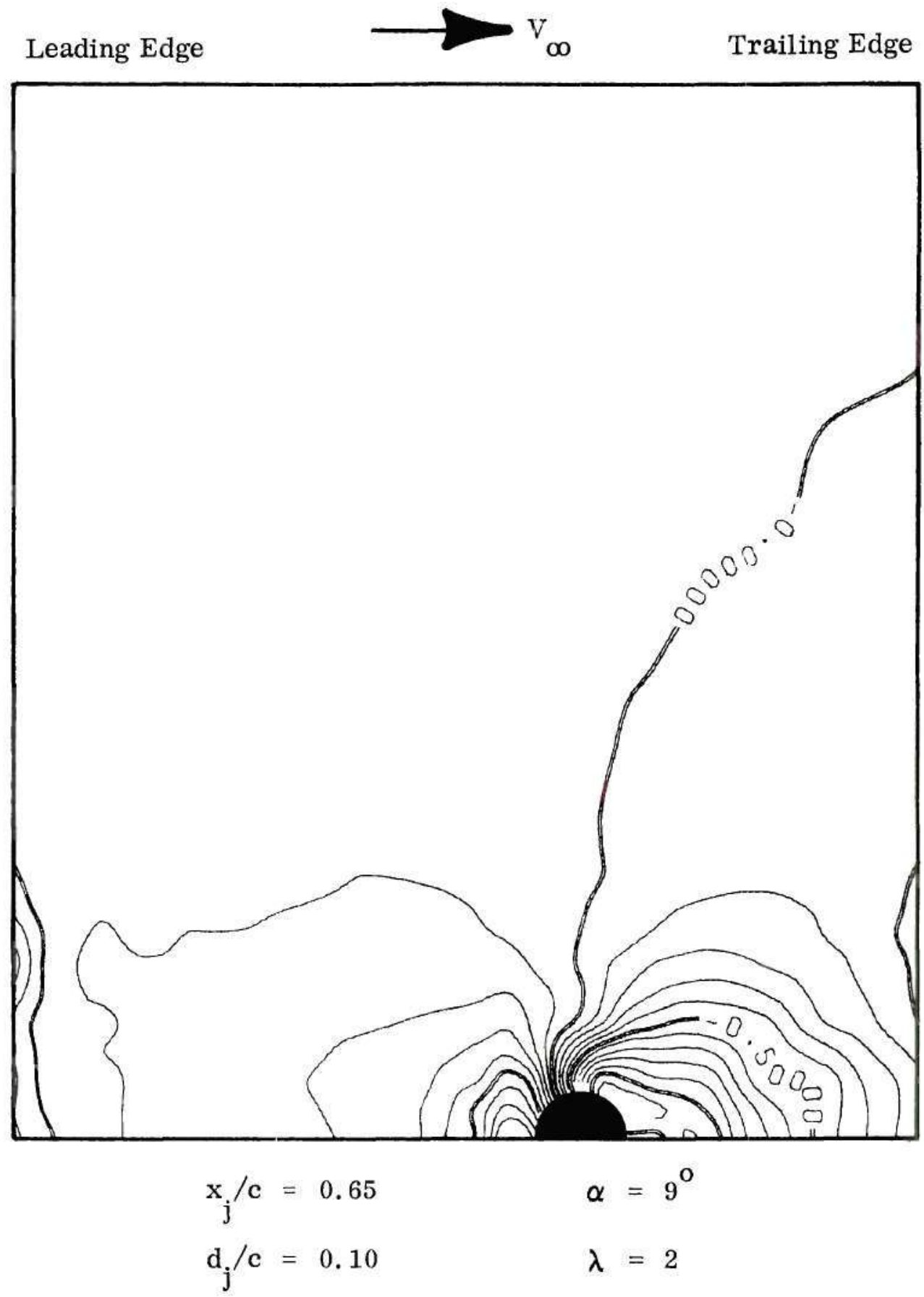

Lower Surface $\quad$ Run No. 67

Figure C-3h. Interference Surface Pressure Distribution. 

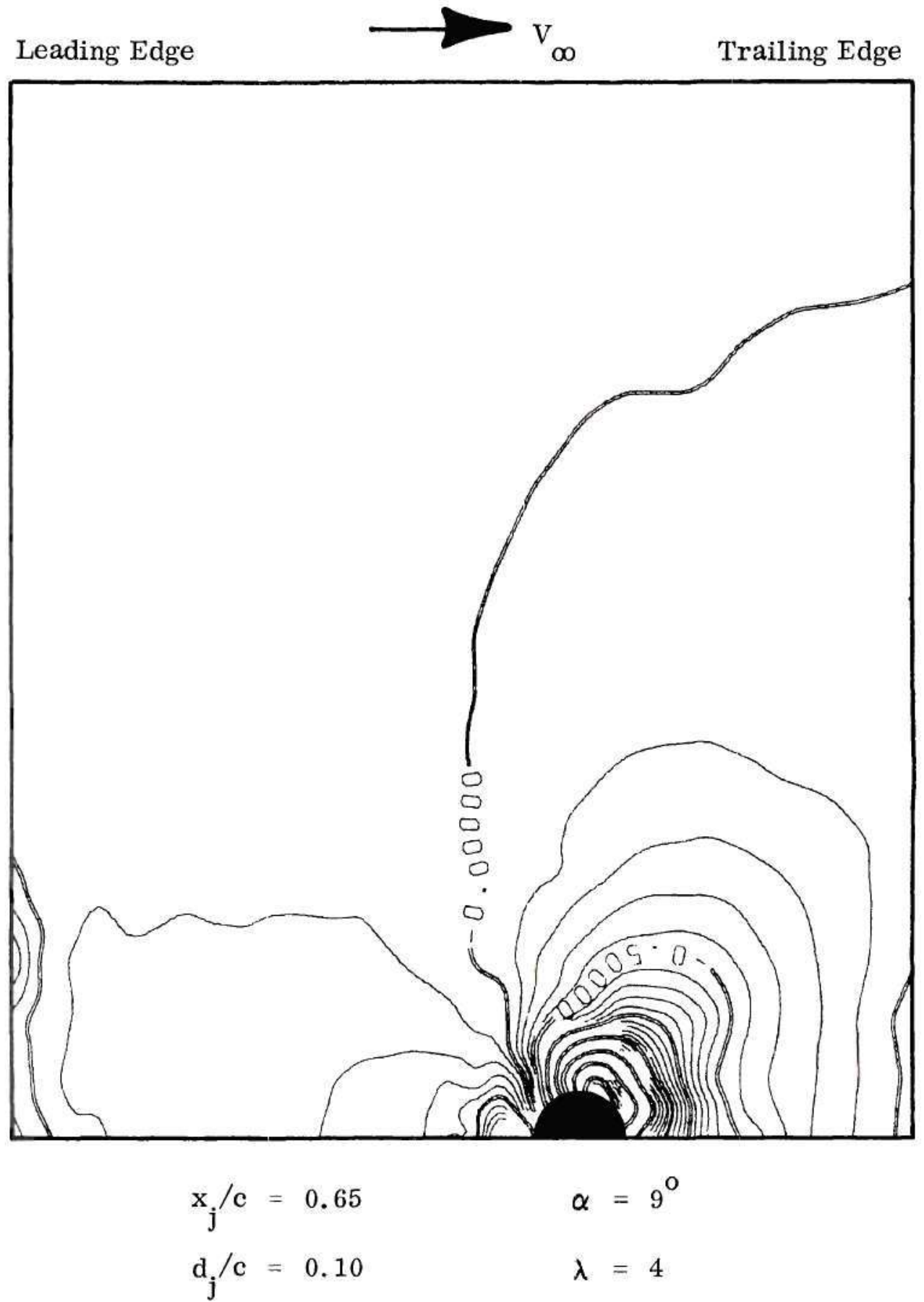

Lower Surface Run No. 65

Figure C-3i. Interference Surface Pressure Distribution. 

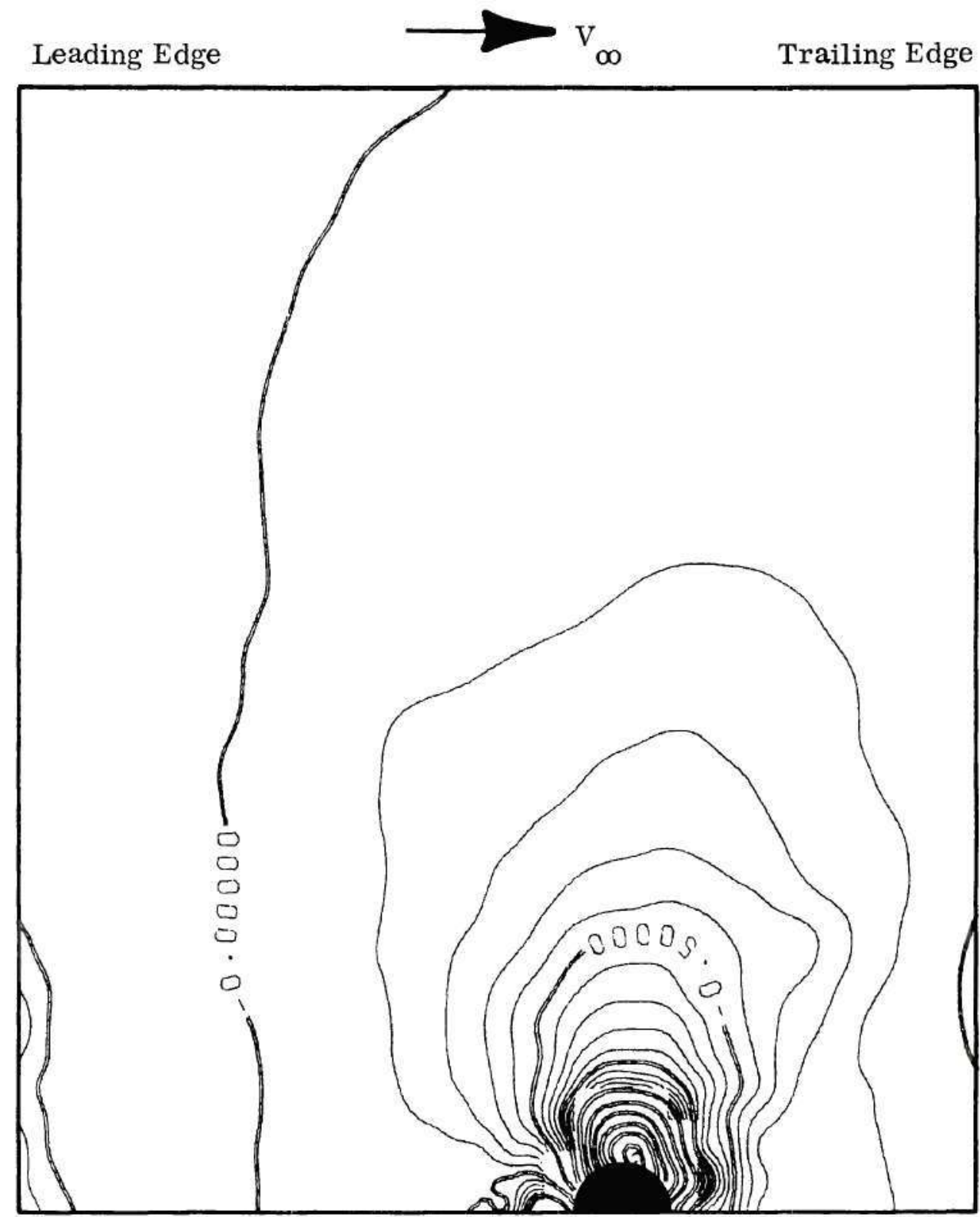

$$
\begin{array}{ll}
\mathrm{x}_{\mathrm{j}} / \mathrm{c}=0.65 & \alpha=9^{\circ} \\
\mathrm{d}_{\mathrm{j}} / \mathrm{c}=0.10 & \lambda=8
\end{array}
$$

Lower Surface

Run No. 64

Figure C-3j. Interference Surface Pressure Distribution. 


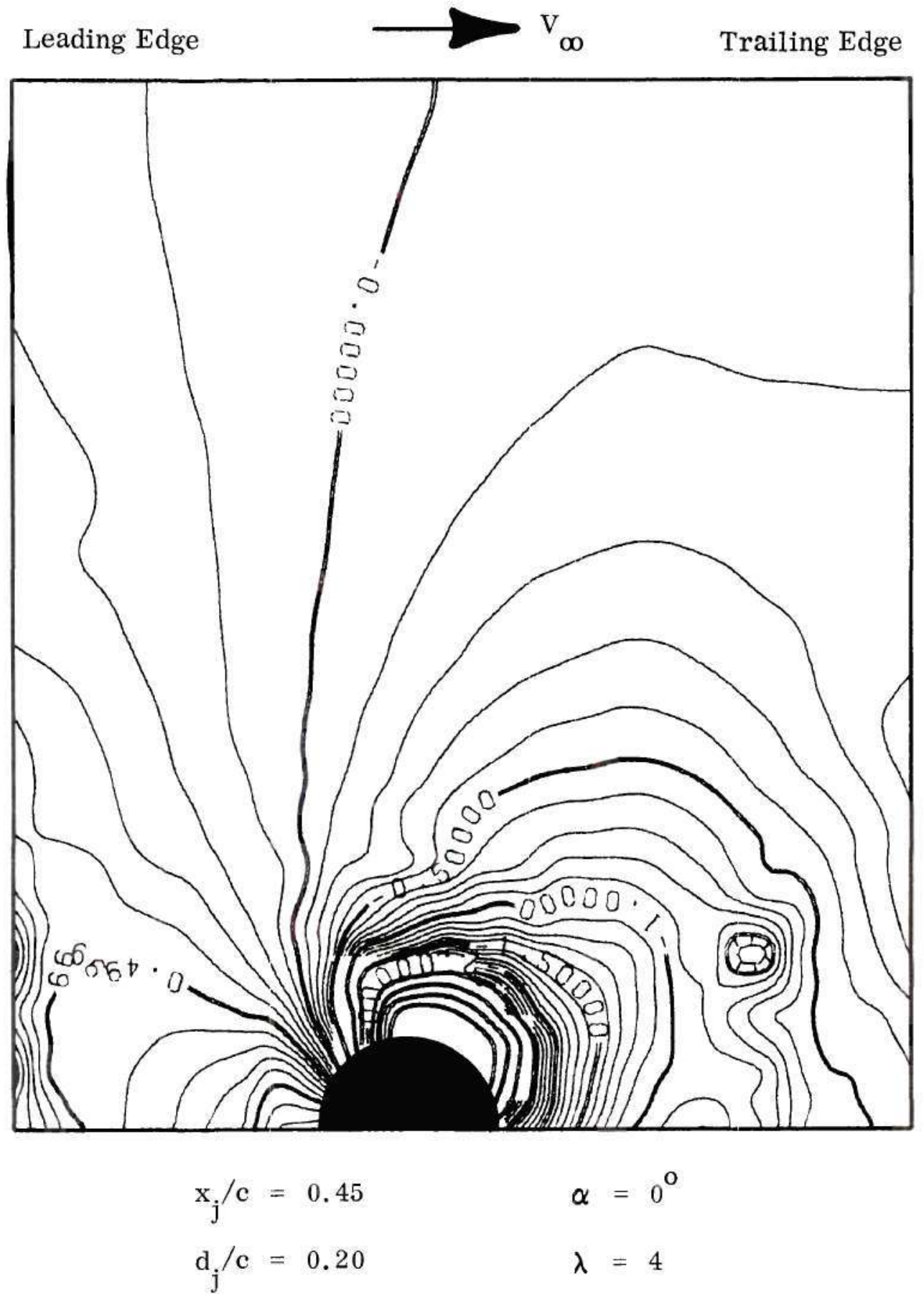

Lower Surface Run No. 80

Figure C-4a. Interference Surface Pressure Distribution. 

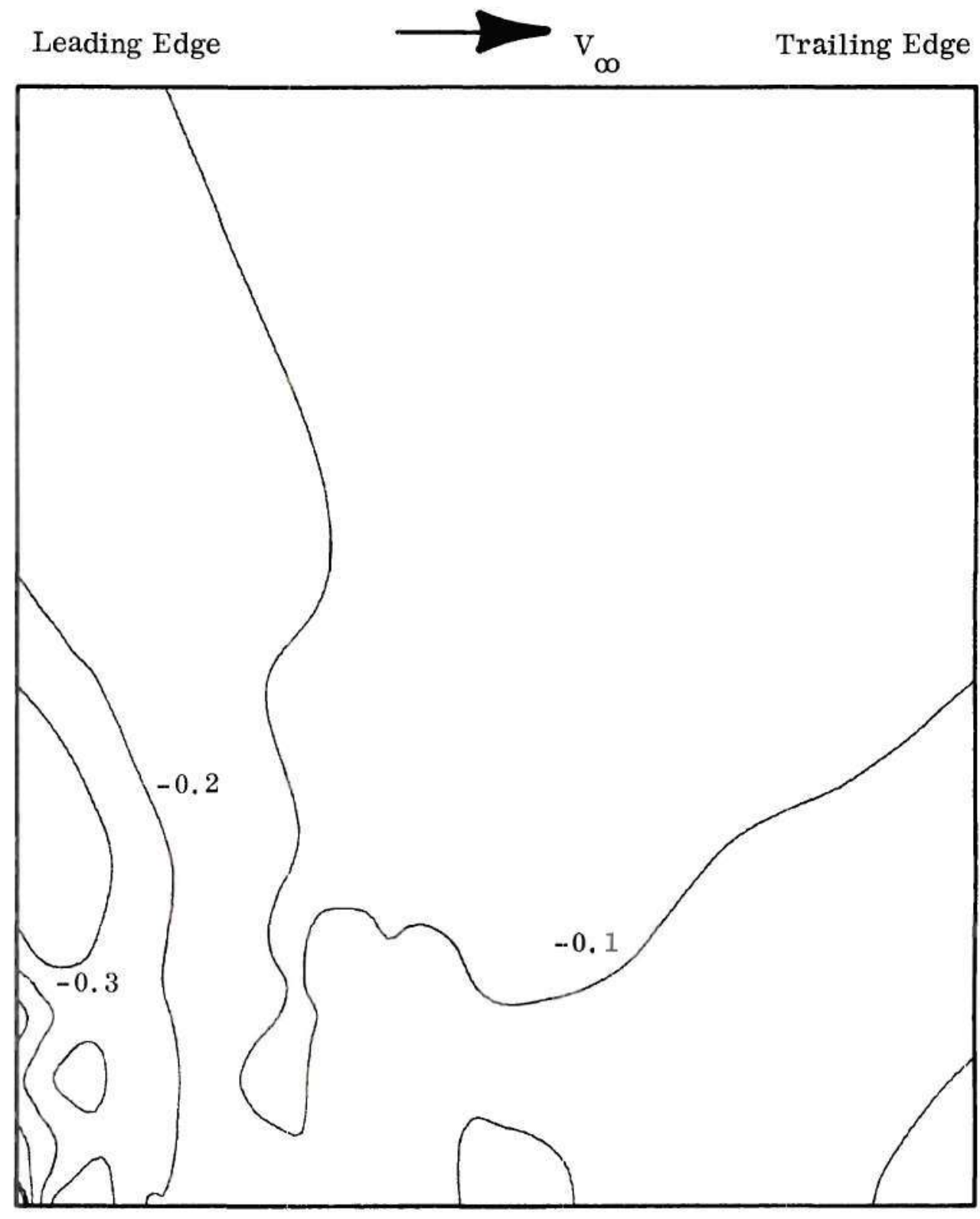
$\mathrm{x}_{\mathrm{j}} / \mathrm{c}=0.45$
$\alpha=0^{0}$
$\mathrm{d}_{\mathrm{j}} / \mathrm{c}=0.20$
$\lambda=4$

Upper Surface

Run No. 80

Figure C-4b. Interference Surface Pressure Distribution. 

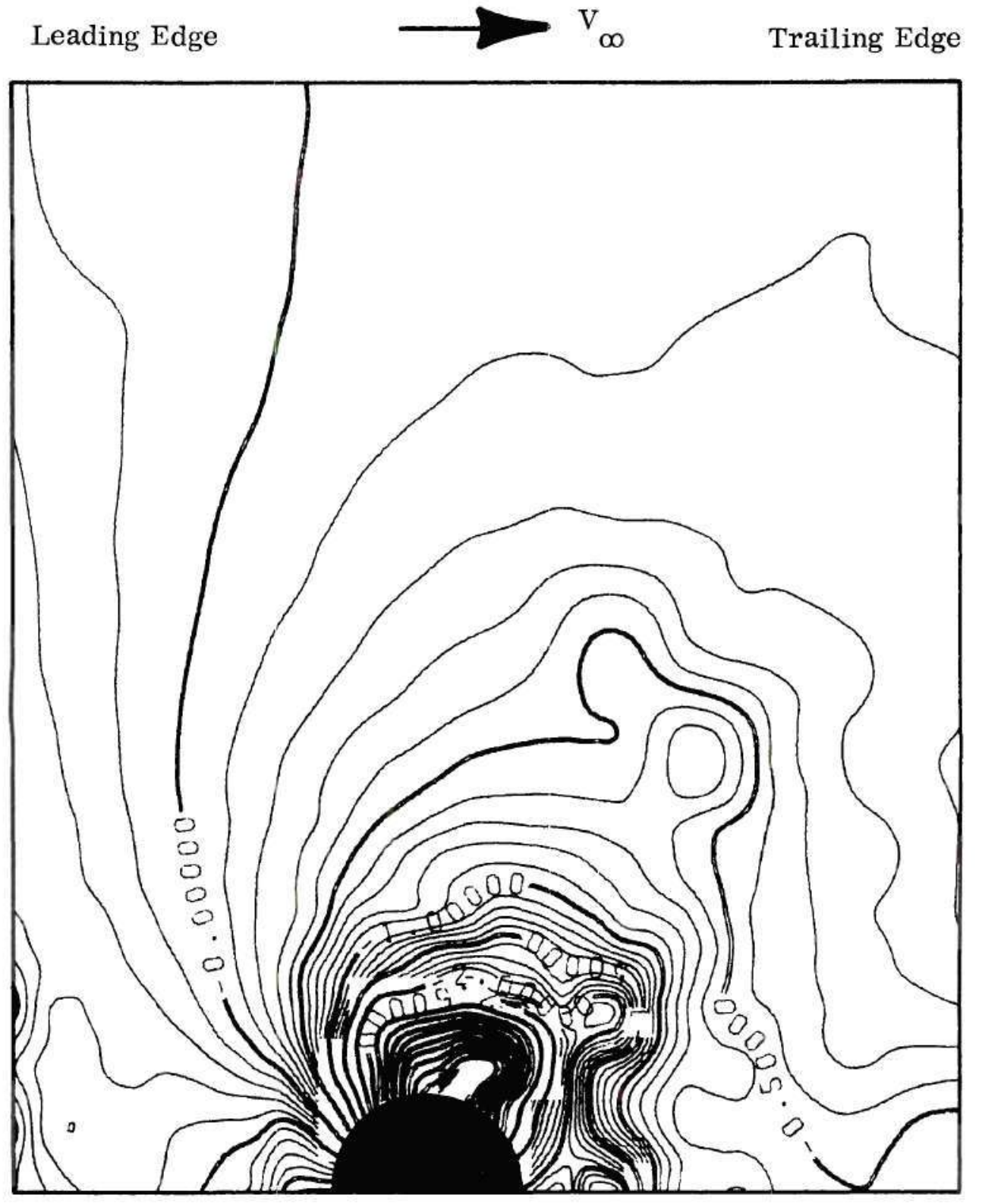

$$
\begin{array}{ll}
\mathrm{x}_{\mathrm{j}} / \mathrm{c}=0.45 & \alpha=0^{\circ} \\
\mathrm{d}_{\mathrm{j}} / \mathrm{c}=0.20 & \lambda=5.9
\end{array}
$$

Lower Surface

Run No. 82

Figure C-4c. Interference Surface Pressure Distribution. 


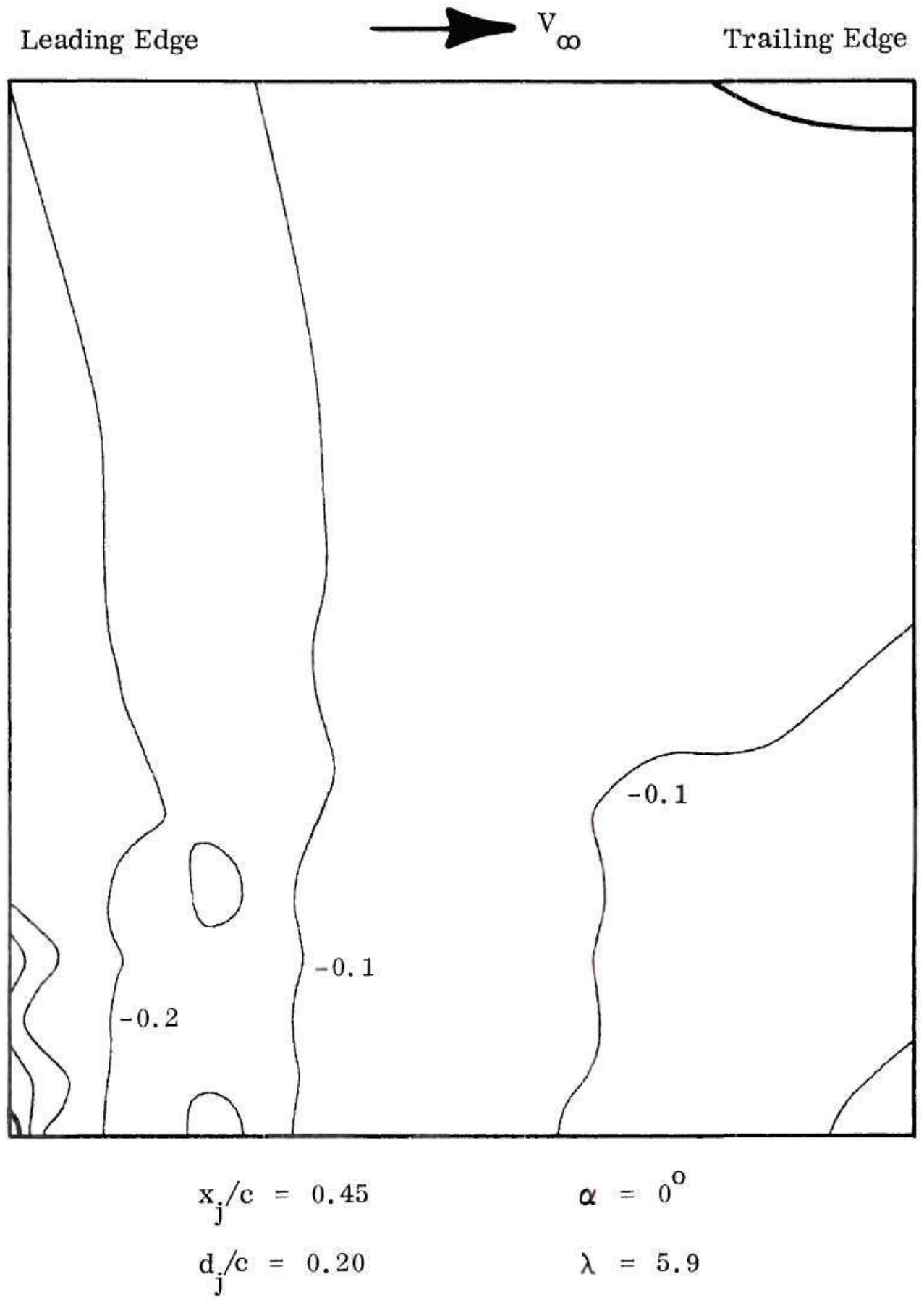

Upper Surface Run No. 82

Figure C-4d. Interference Surface Pressure Distribution. 

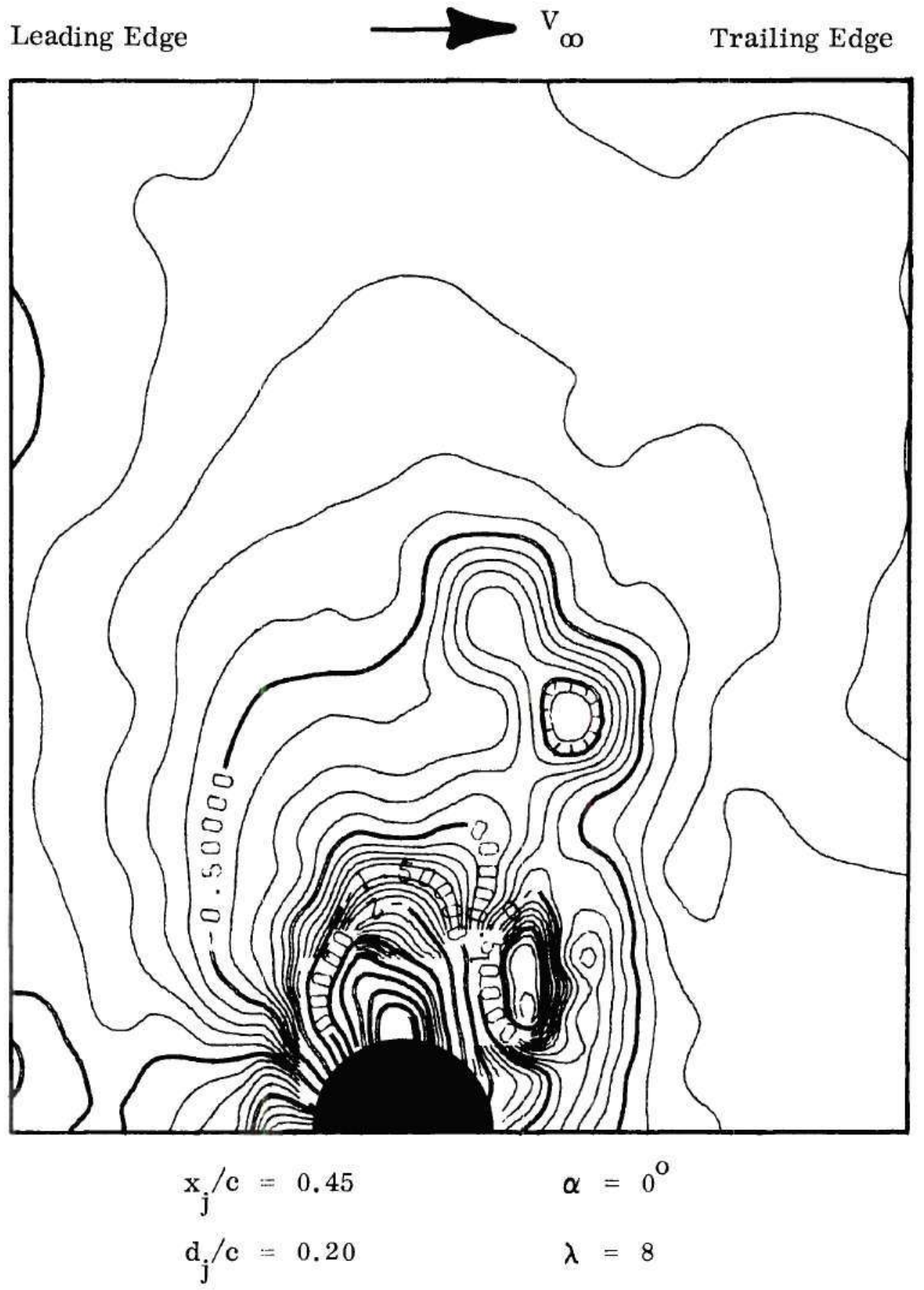

Lower Surface Run No. 79

Figure C-4e. Interference Surface Pressure Distribution. 


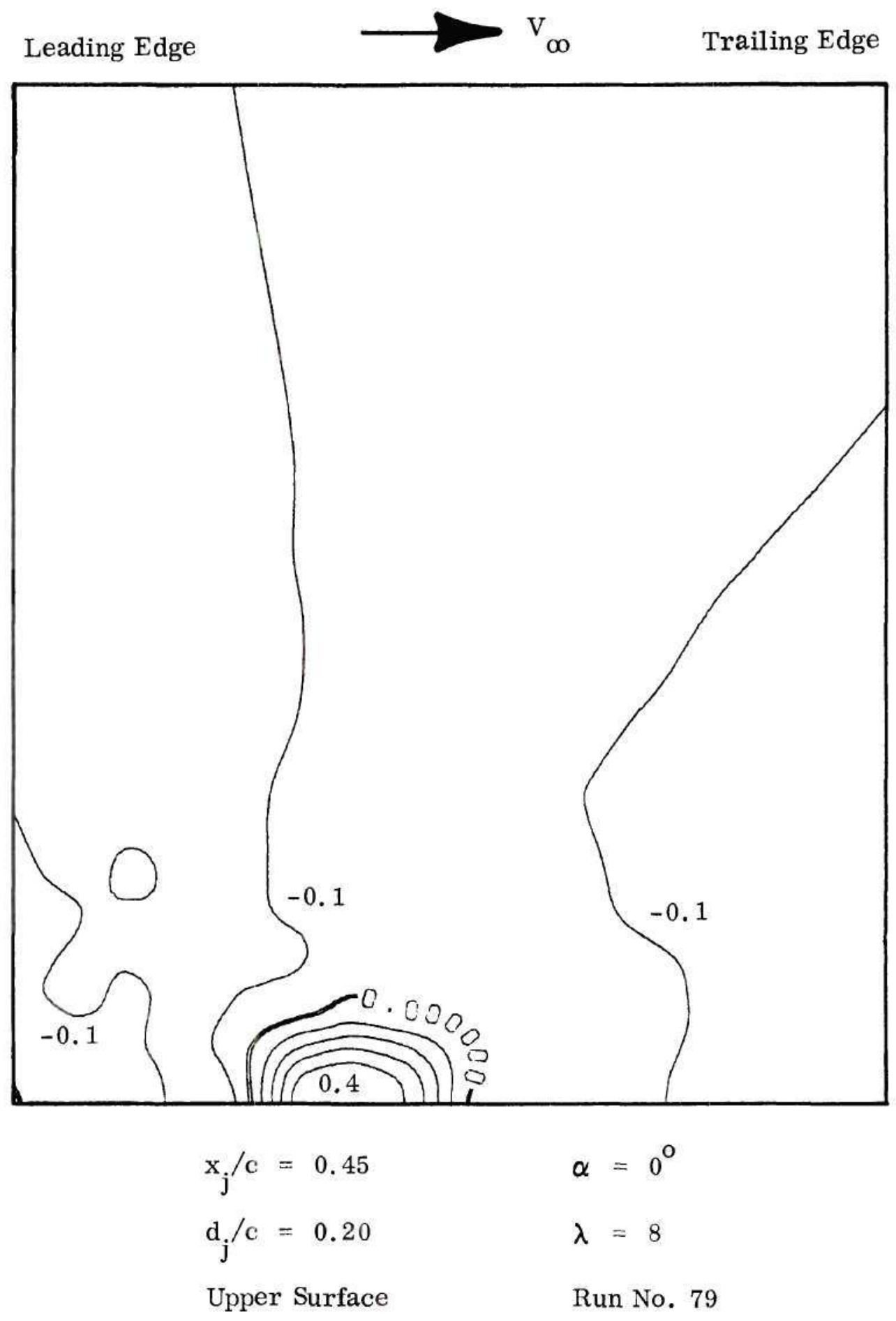

Figure C-4f. Interference Surface Pressure Distribution. 

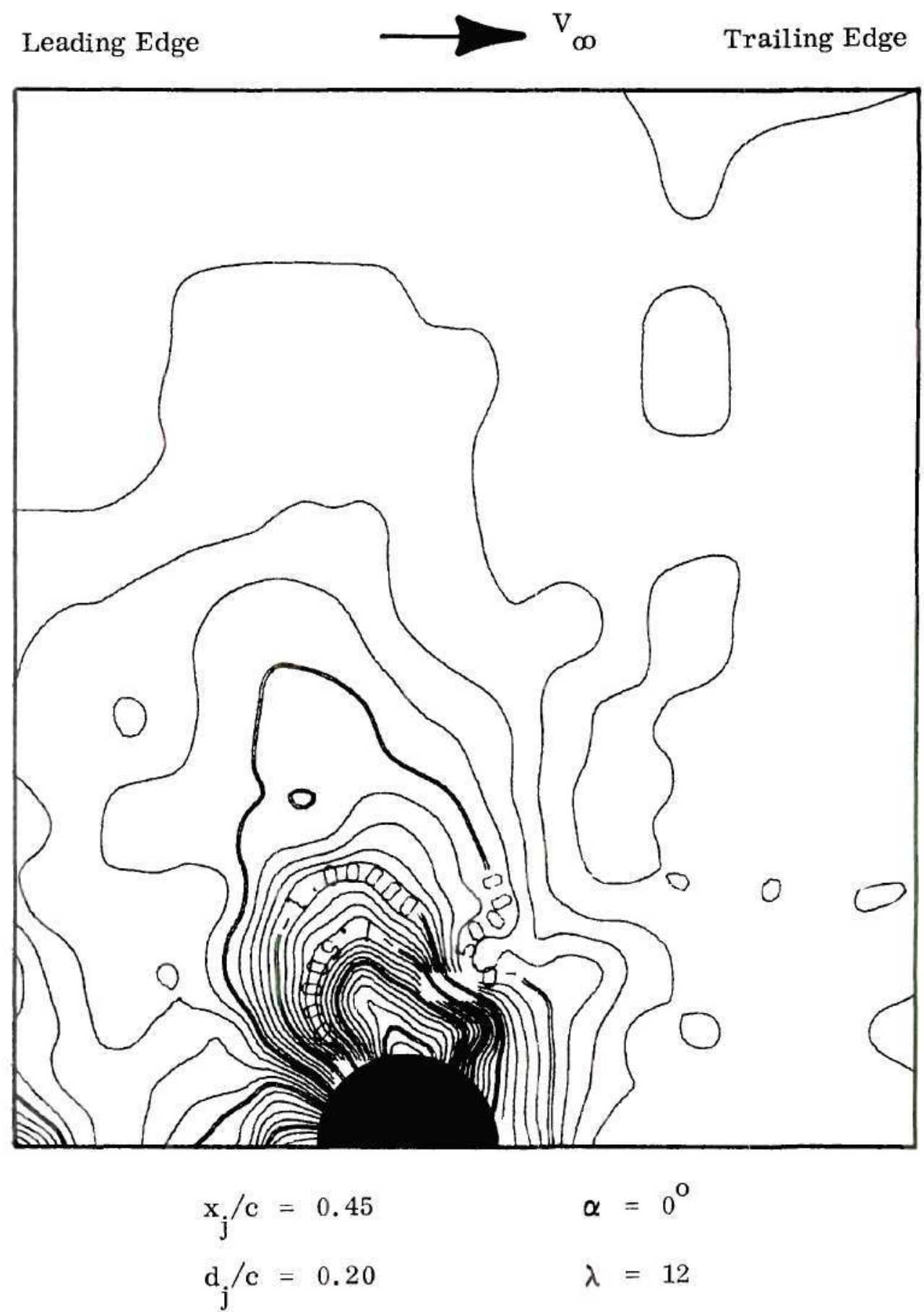

Lower Surface

Run No. 81

Figure C-4g. Interference Surface Pressure Distribution. 


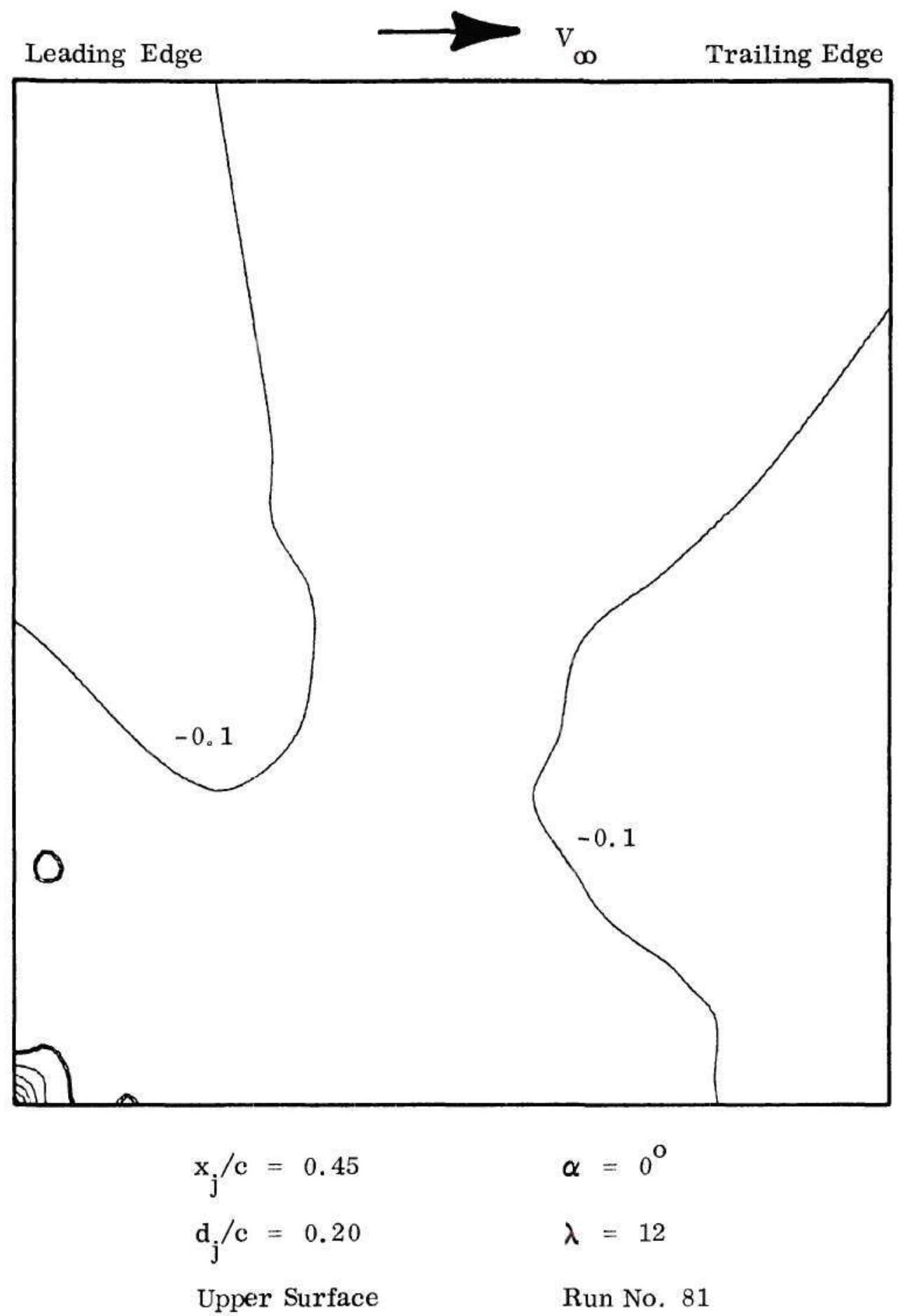

Figure C-4h. Interference Surface Pressure Distribution. 


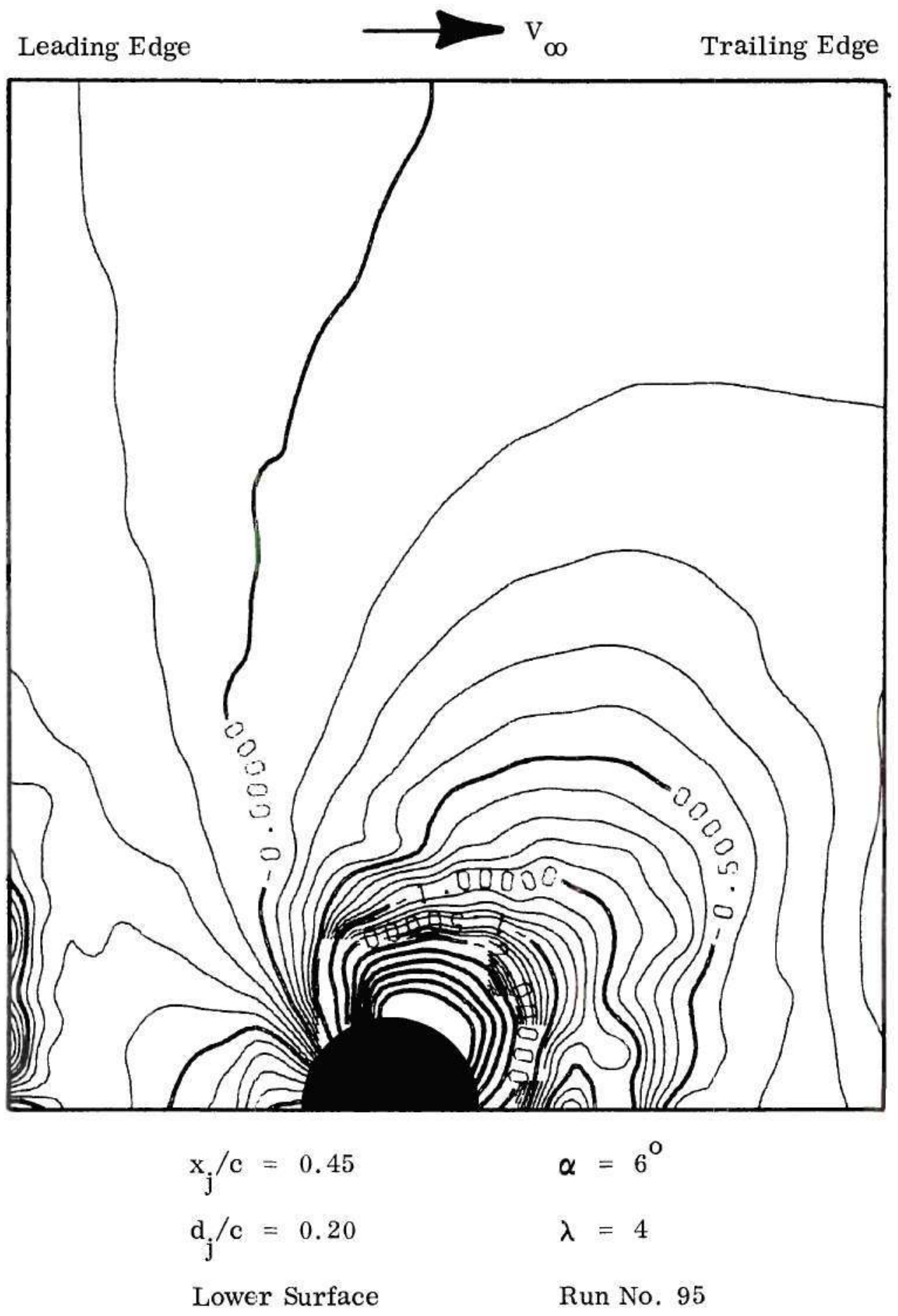

Figure C-4i. Interference Surface Pressure Distribution. 

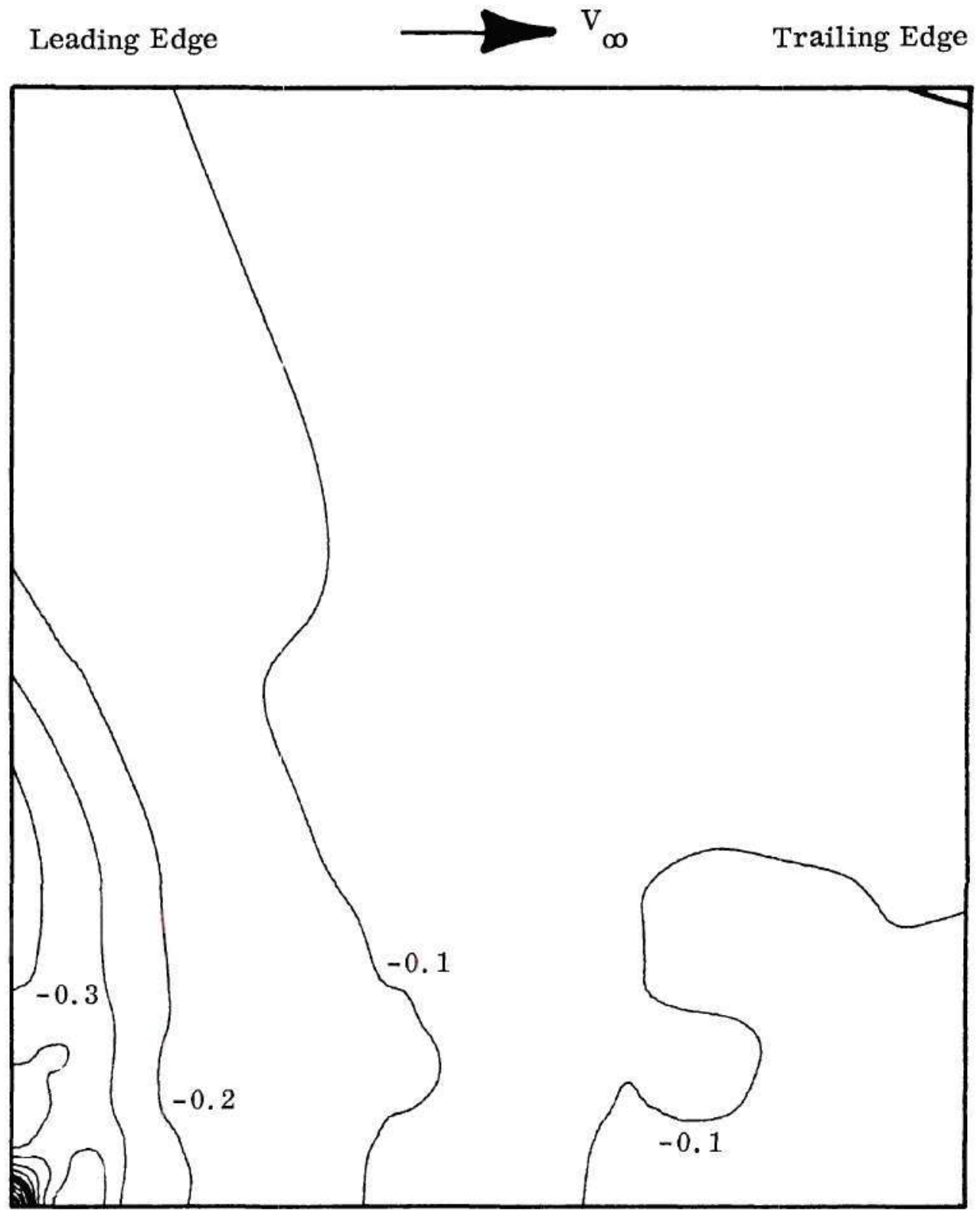

$$
\begin{array}{ll}
\mathrm{x}_{\mathrm{j}} / \mathrm{c}=0.45 & \alpha=6^{\circ} \\
\mathrm{d}_{\mathrm{j}} / \mathrm{c}=0.20 & \lambda=4
\end{array}
$$

Upper Surface Run No. 95

Figure C-4j. Interference Surface Pressure Distribution. 


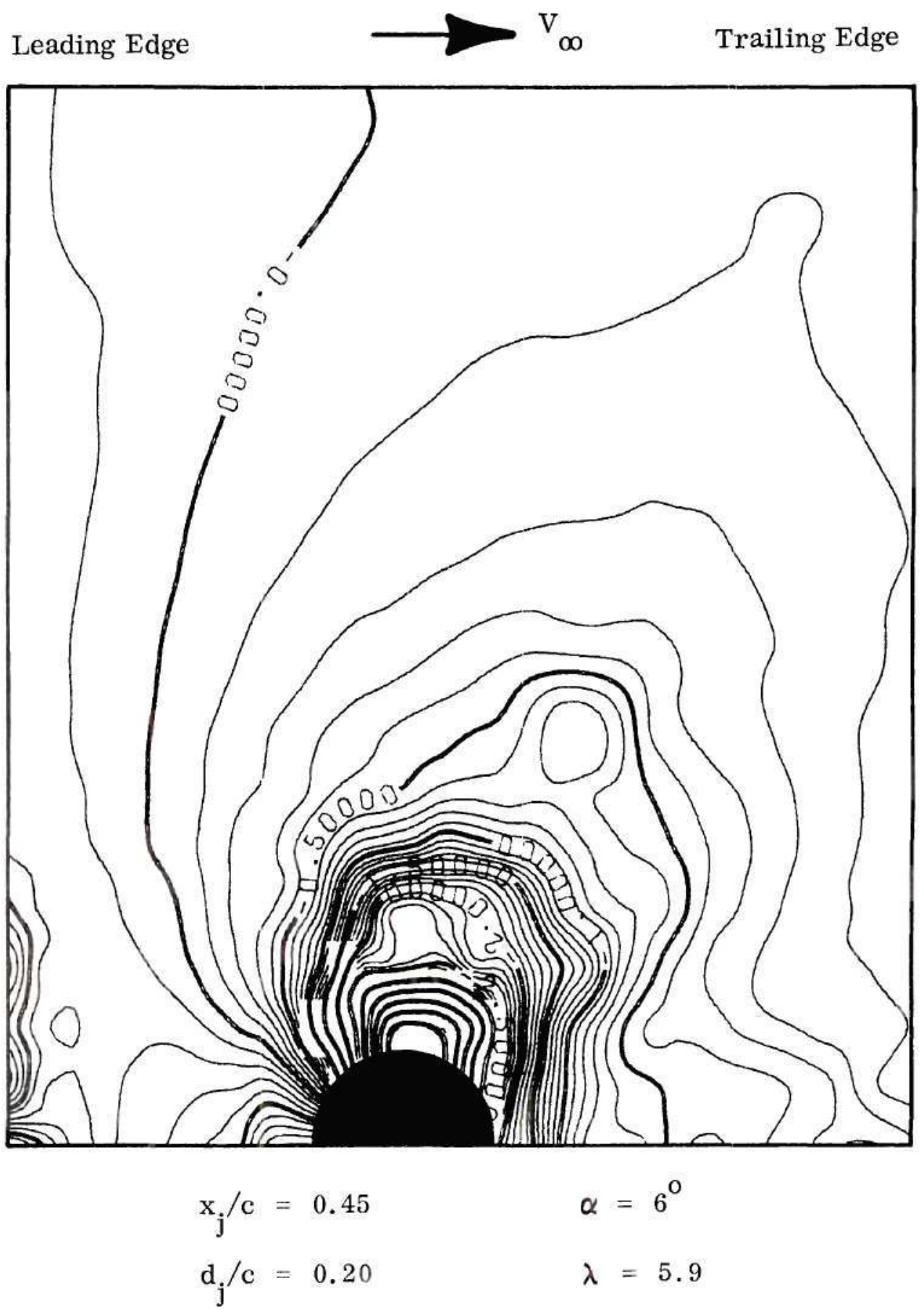

Lower Surface $\quad$ Run No. 96

Figure C-4k. Interference Surface Pressure Distribution. 


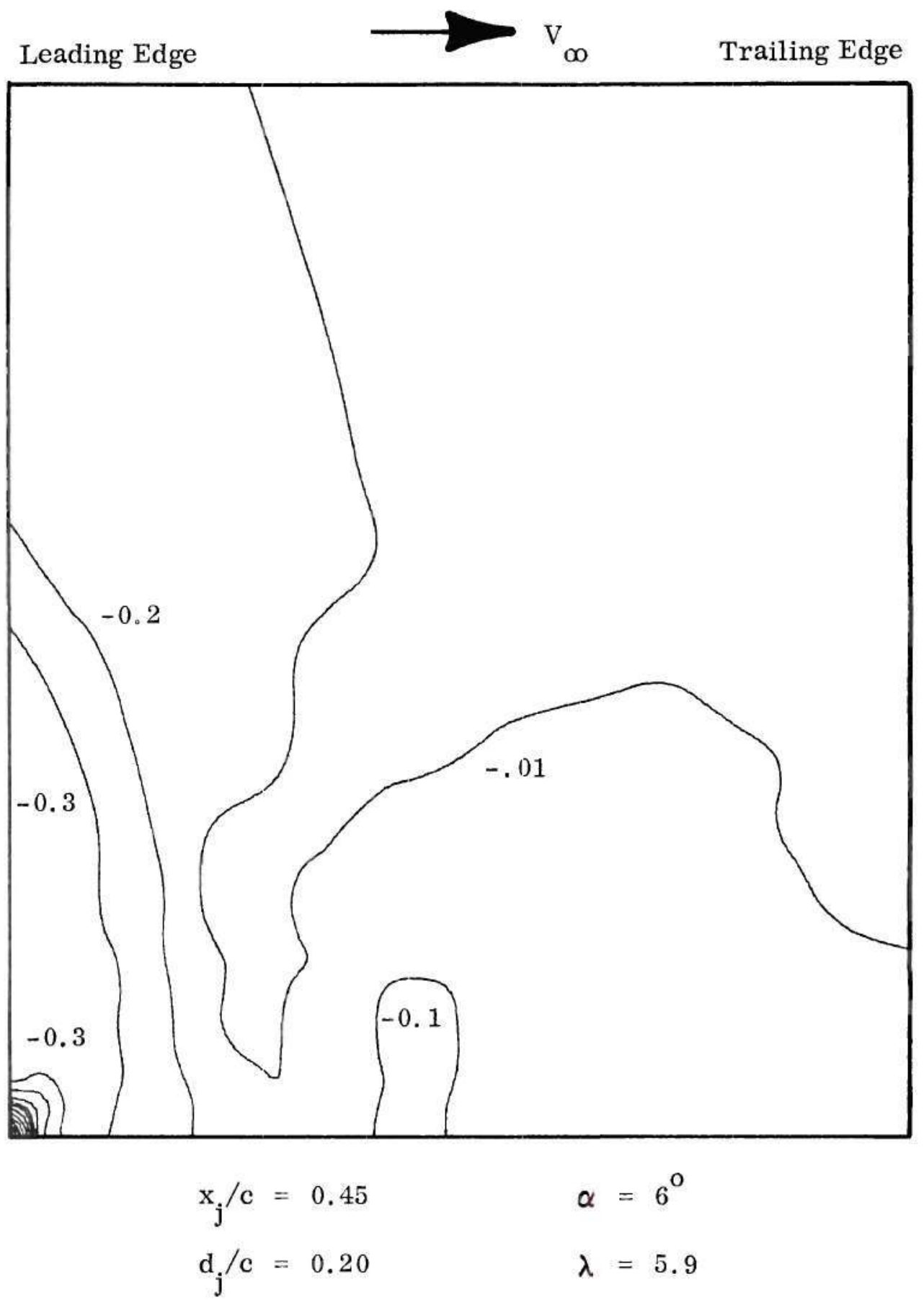

Upper Surface Run No. 96

Figure C-41. Interference Surface Pressure Distribution. 


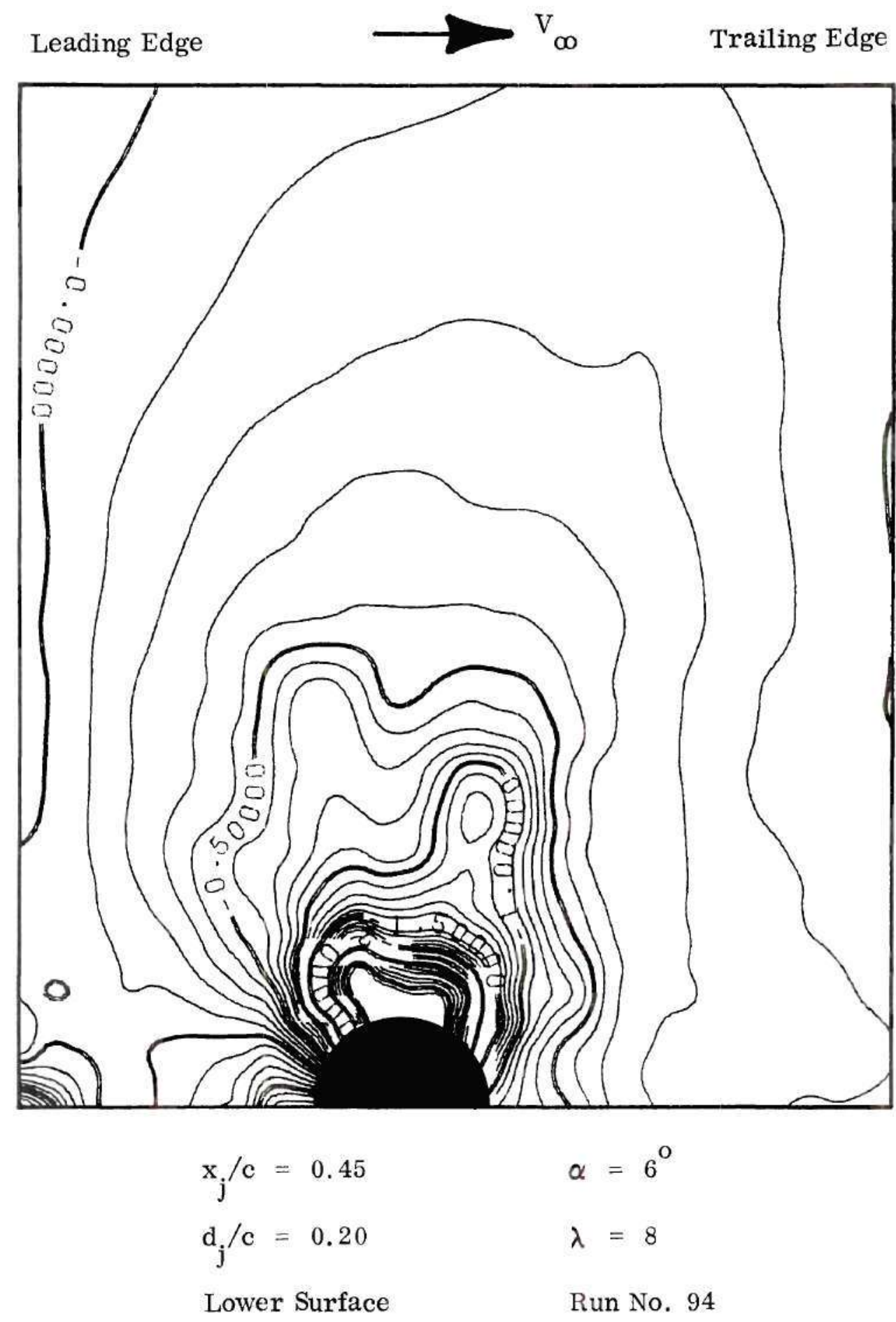

Figure C-4m. Interference Surface Pressure Distribution. 


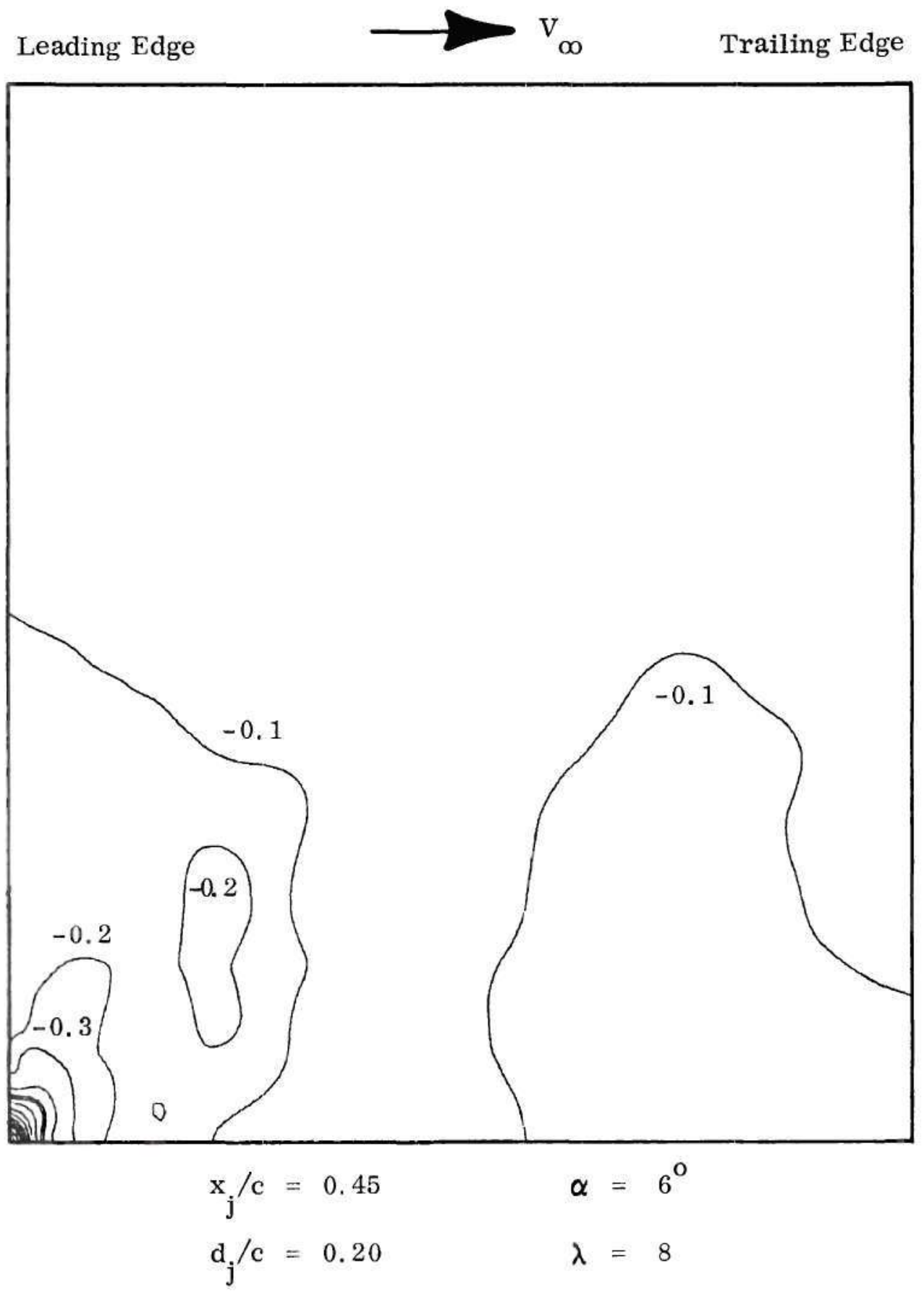

Upper Surface

Run No. 94

Figure C-4n. Interference Surface Pressure Distribution. 


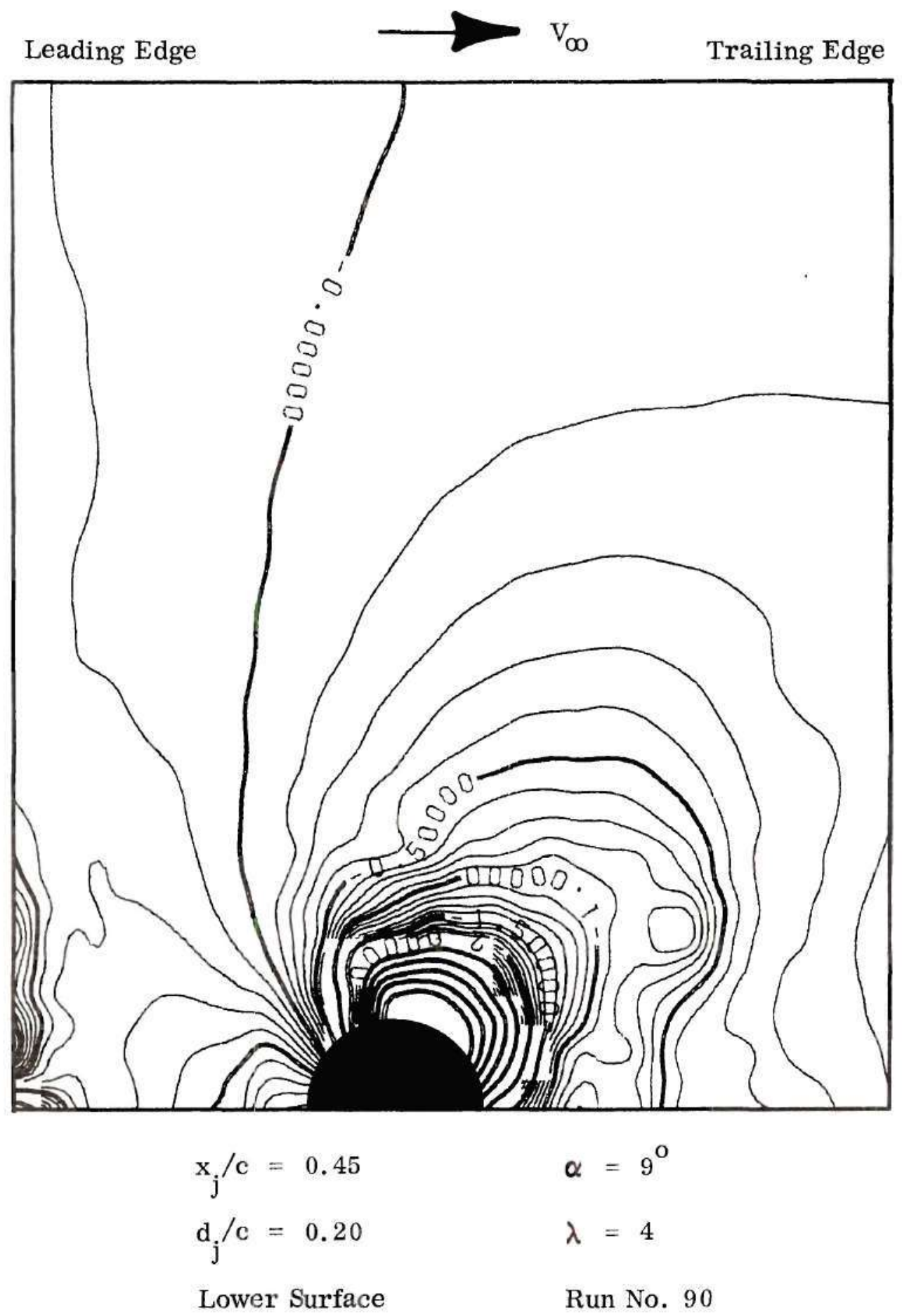

Figure C-4o. Interference Surface Pressure Distribution. 


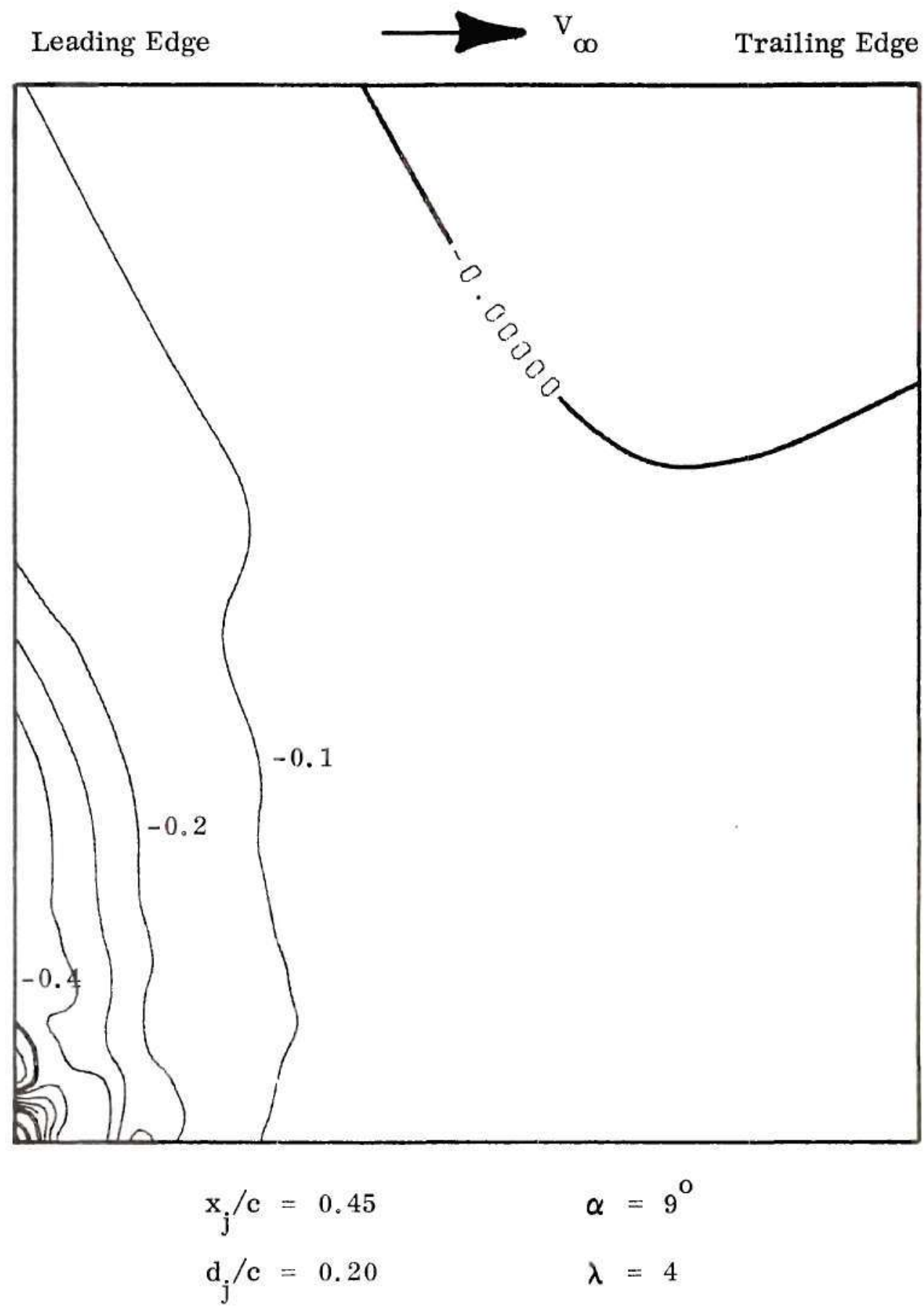

Upper Surface Run No. 90

Figure C-4p. Interference Surface Pressure Distribution. 

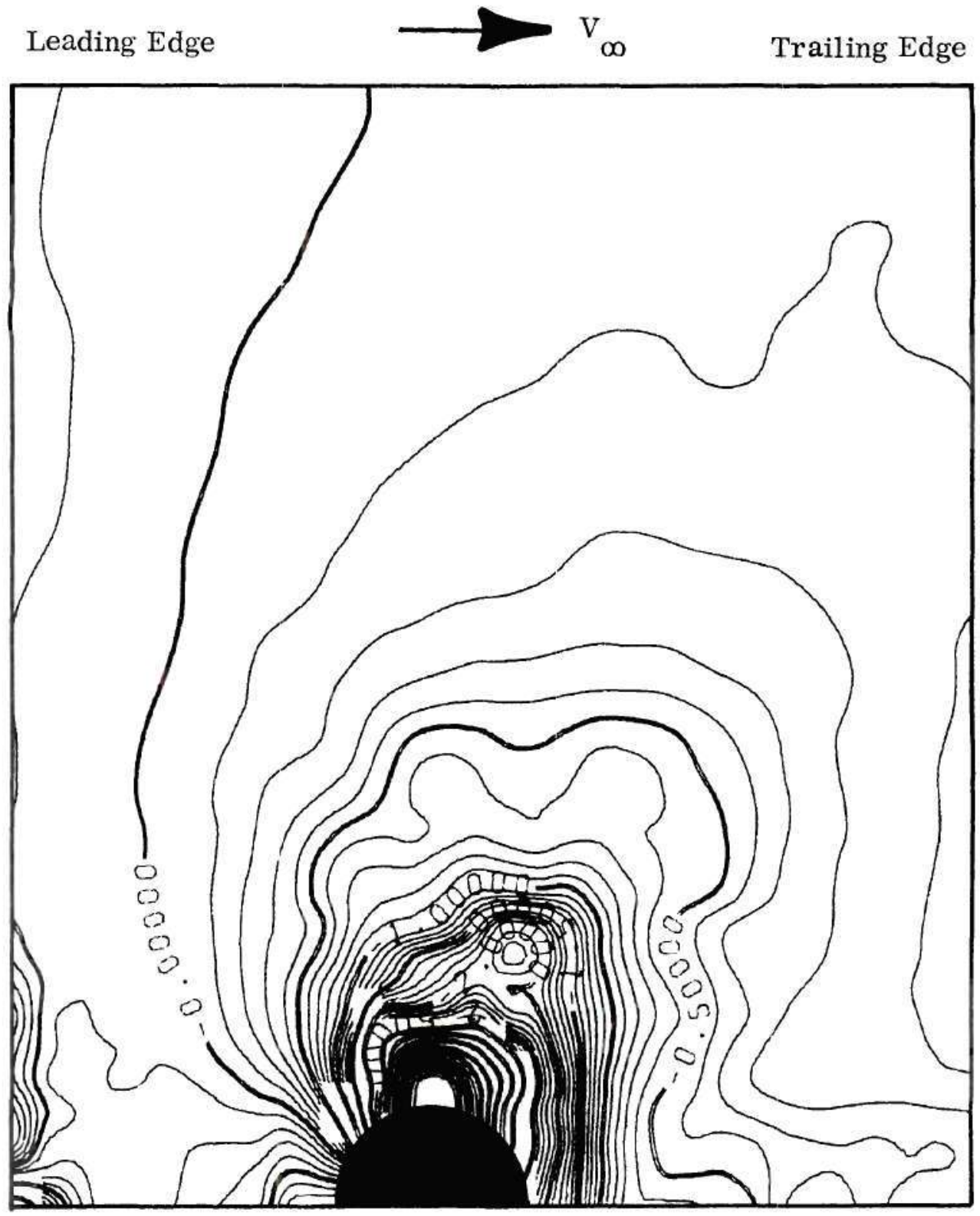

$$
\begin{array}{ll}
\mathrm{x}_{\mathrm{j}} / \mathrm{c}=0.45 & \alpha=9^{\circ} \\
\mathrm{d}_{\mathrm{j}} / \mathrm{c}=0.20 & \lambda=5.9
\end{array}
$$

Lower Surface

Run No. 91

Figure C-4q. Interference Surface Pressure Distribution 


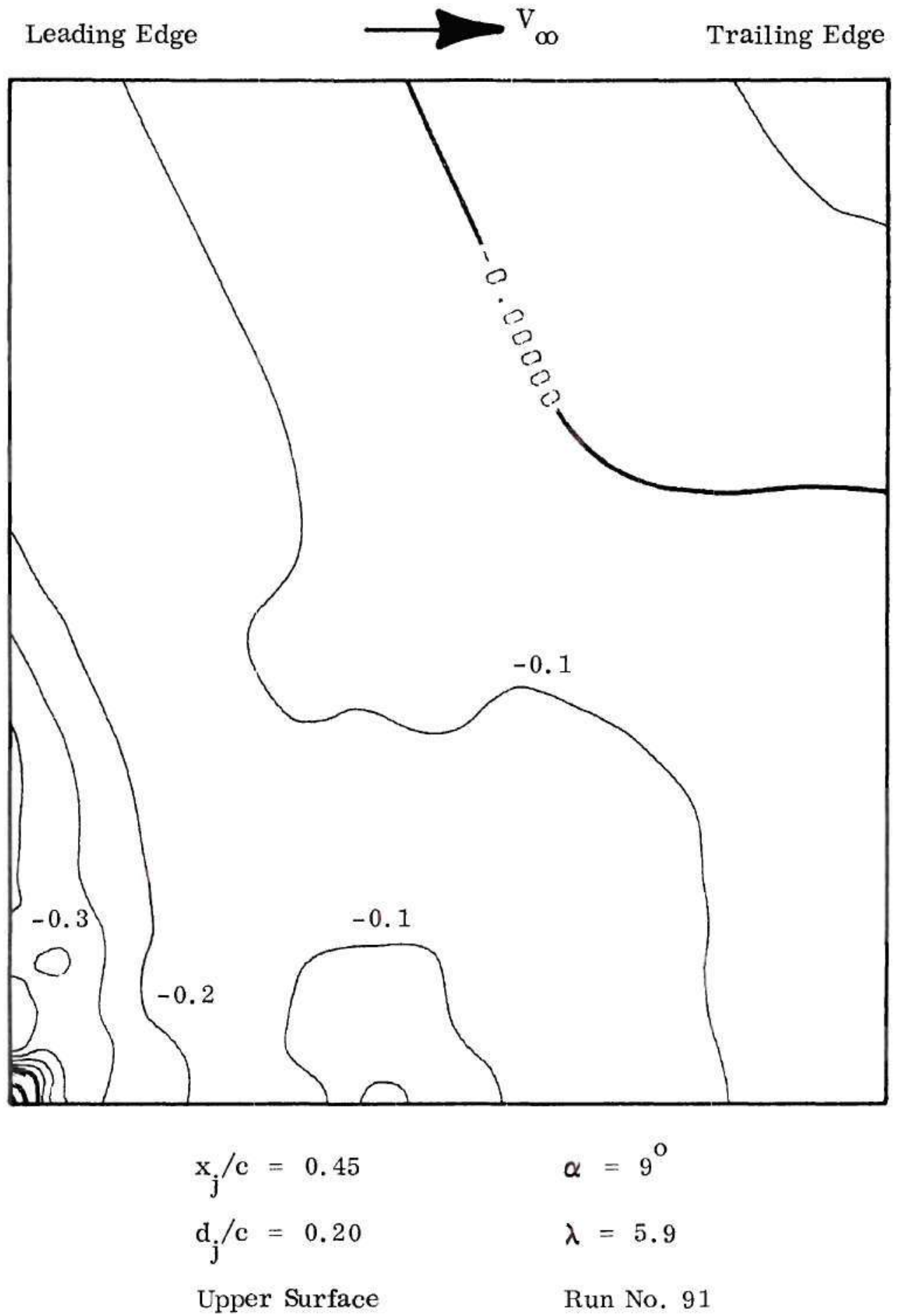

Figure C-4r. Interference Surface Pressure Distribution. 


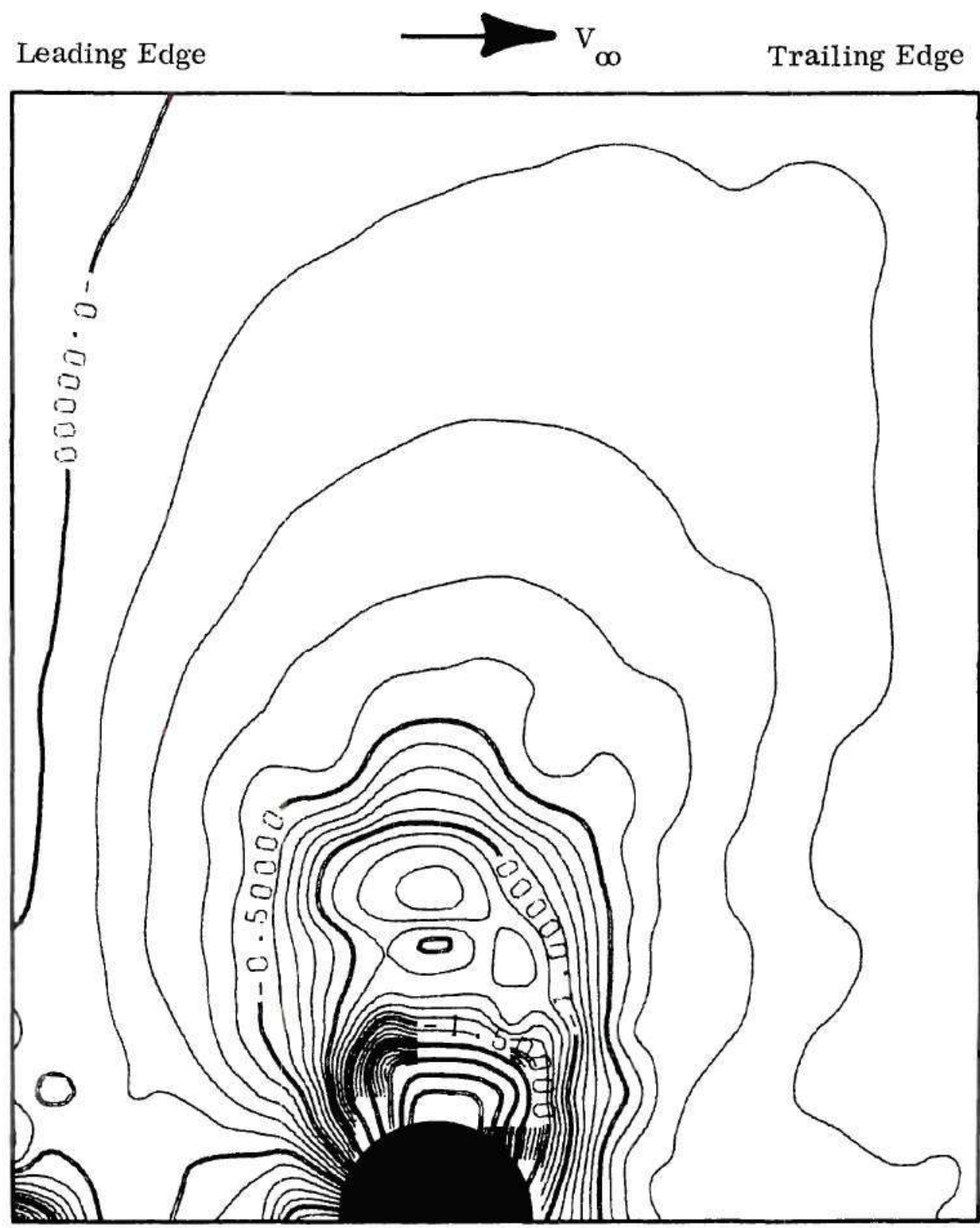

$$
\begin{array}{ll}
\mathrm{x}_{\mathrm{j}} / \mathrm{c}=0.45 & \alpha=9^{\circ} \\
\mathrm{d}_{\mathrm{j}} / \mathrm{c}=0.20 & \lambda=8
\end{array}
$$

Lower Surface

Run No. 89

Figure C-4s. Interference Surface Pressure Distribution. 


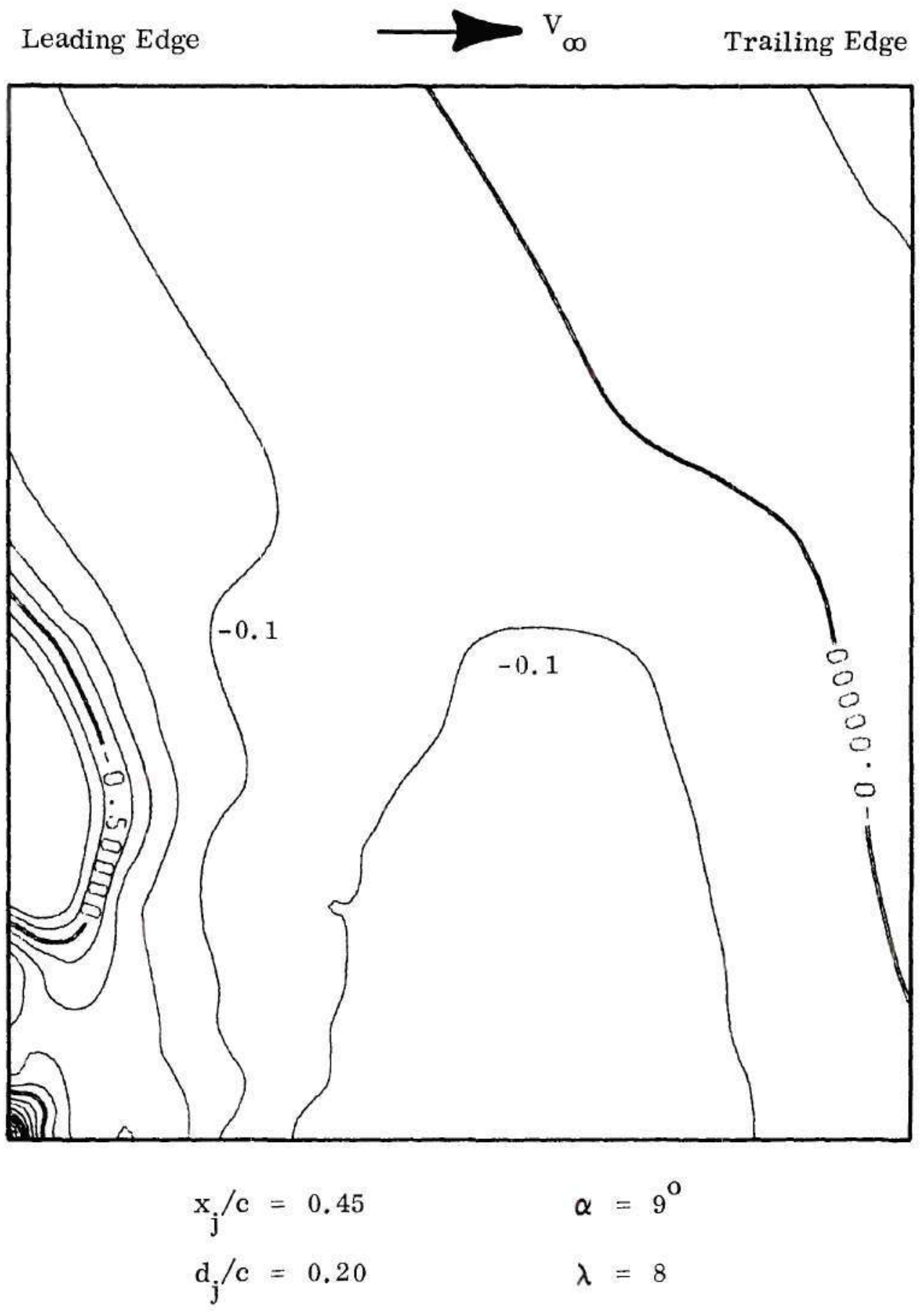

Upper Surface Run No. 89

Figure C-4t. Interference Surface Pressure Distribution. 

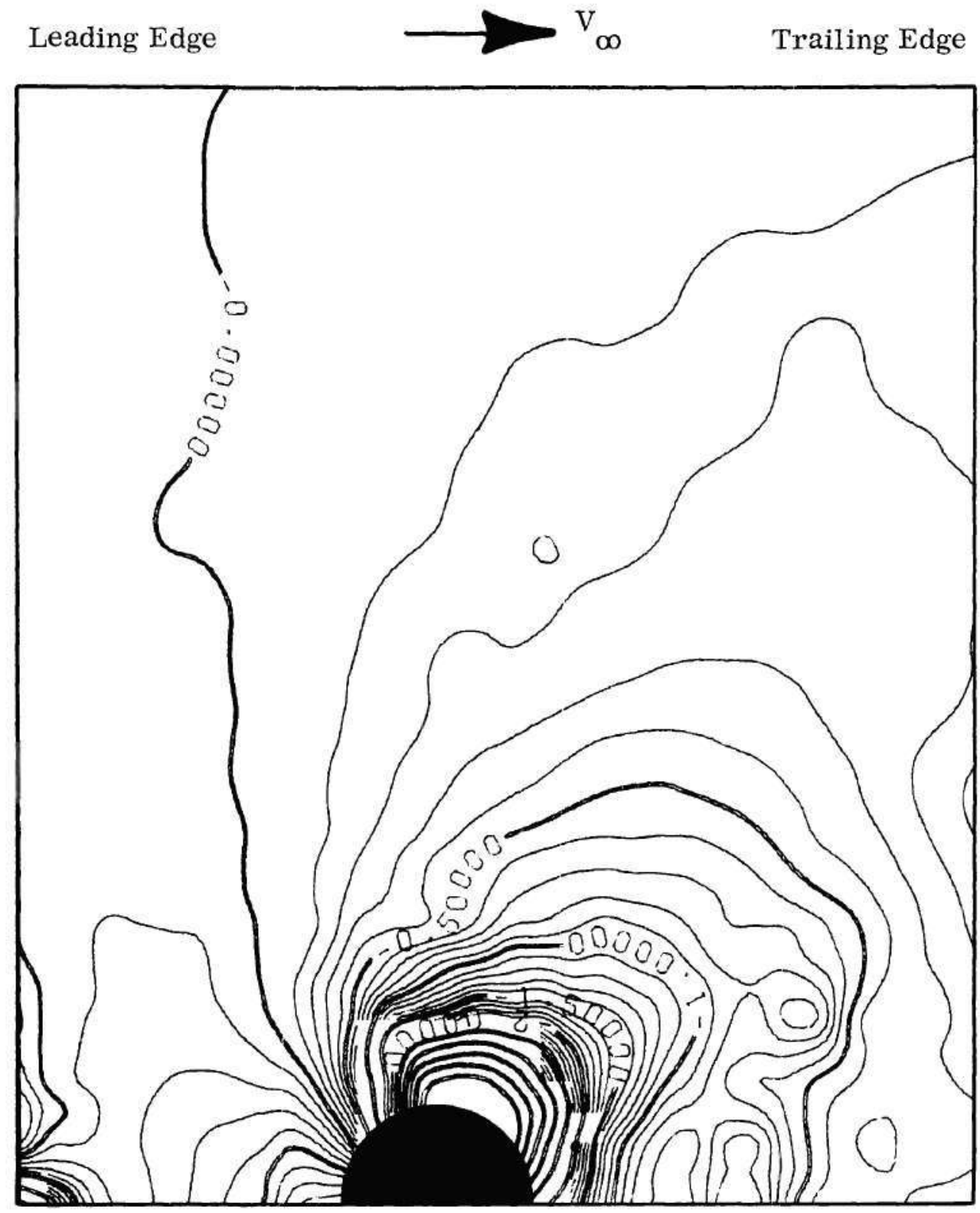

$$
\begin{array}{ll}
\mathrm{x}_{\mathrm{j}} / \mathrm{c}=0.45 & \alpha=12^{\mathrm{O}} \\
\mathrm{d}_{\mathrm{j}} / \mathrm{c}=0.20 & \lambda=4
\end{array}
$$

Lower Surface $\quad$ Run No. 86

Figure C-4u. Interference Surface Pressure Distribution. 


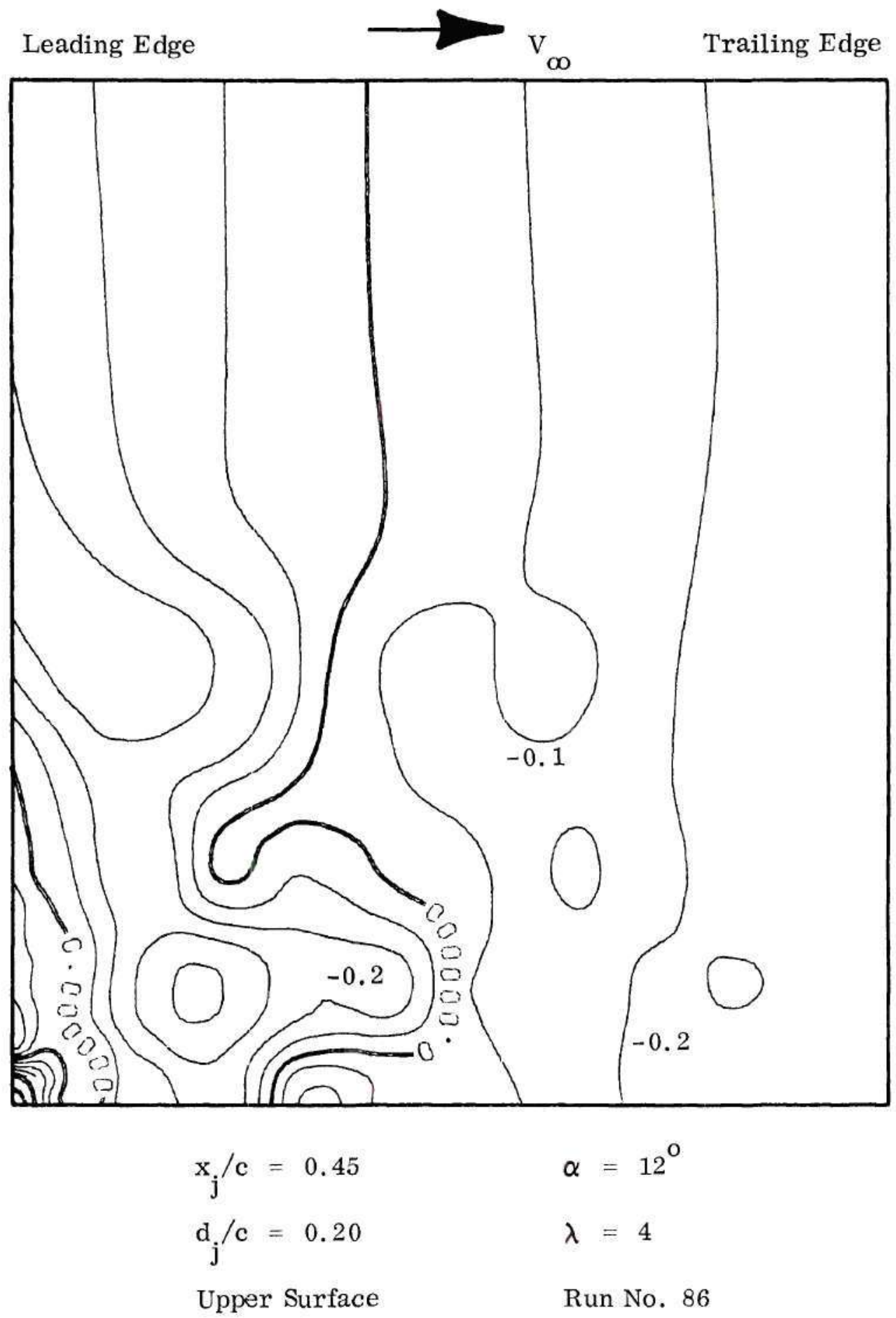

Figure C-4v. Interference Surface Pressure Distribution. 


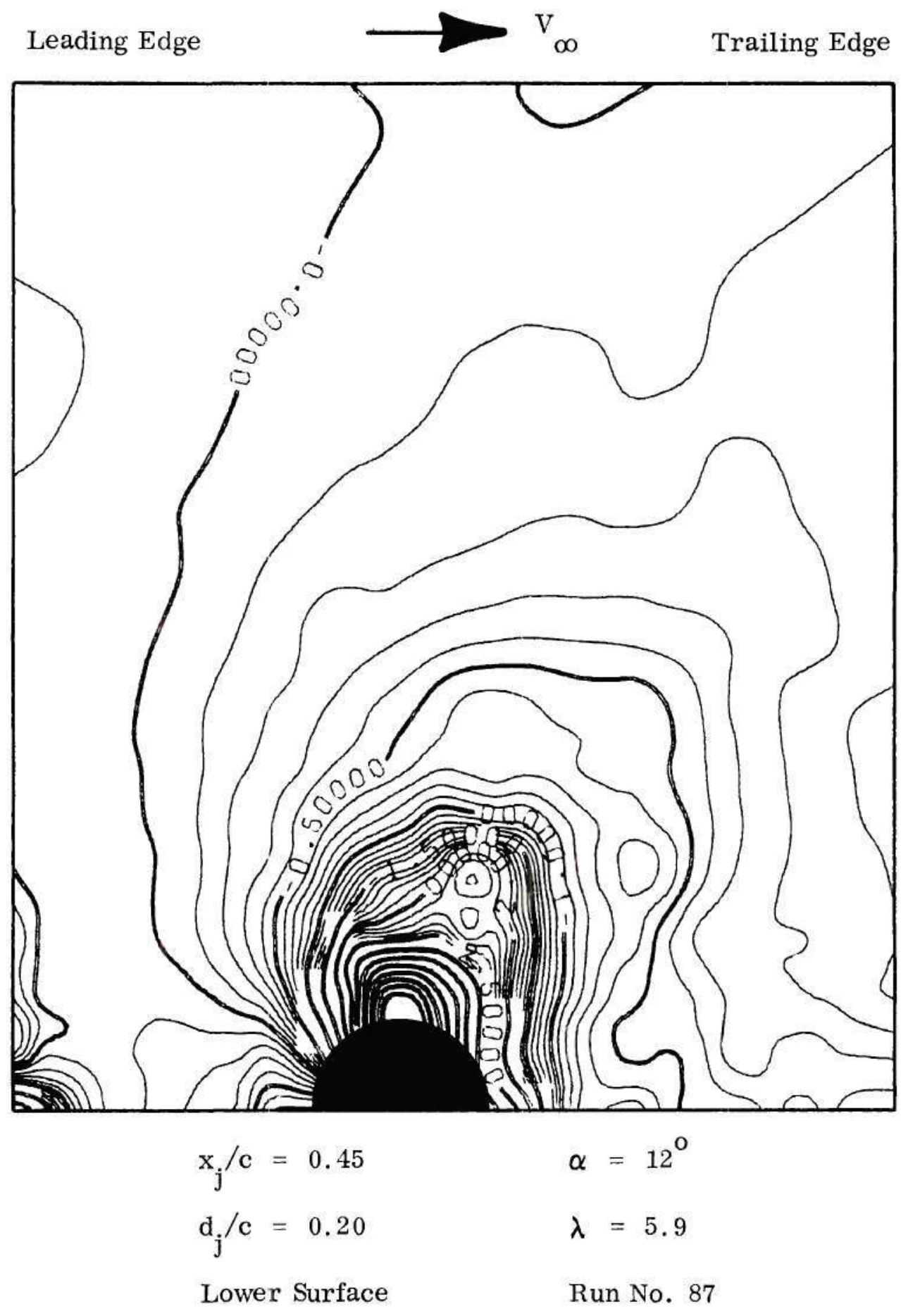

Figure C-4w. Interference Surface Pressure Distribution. 


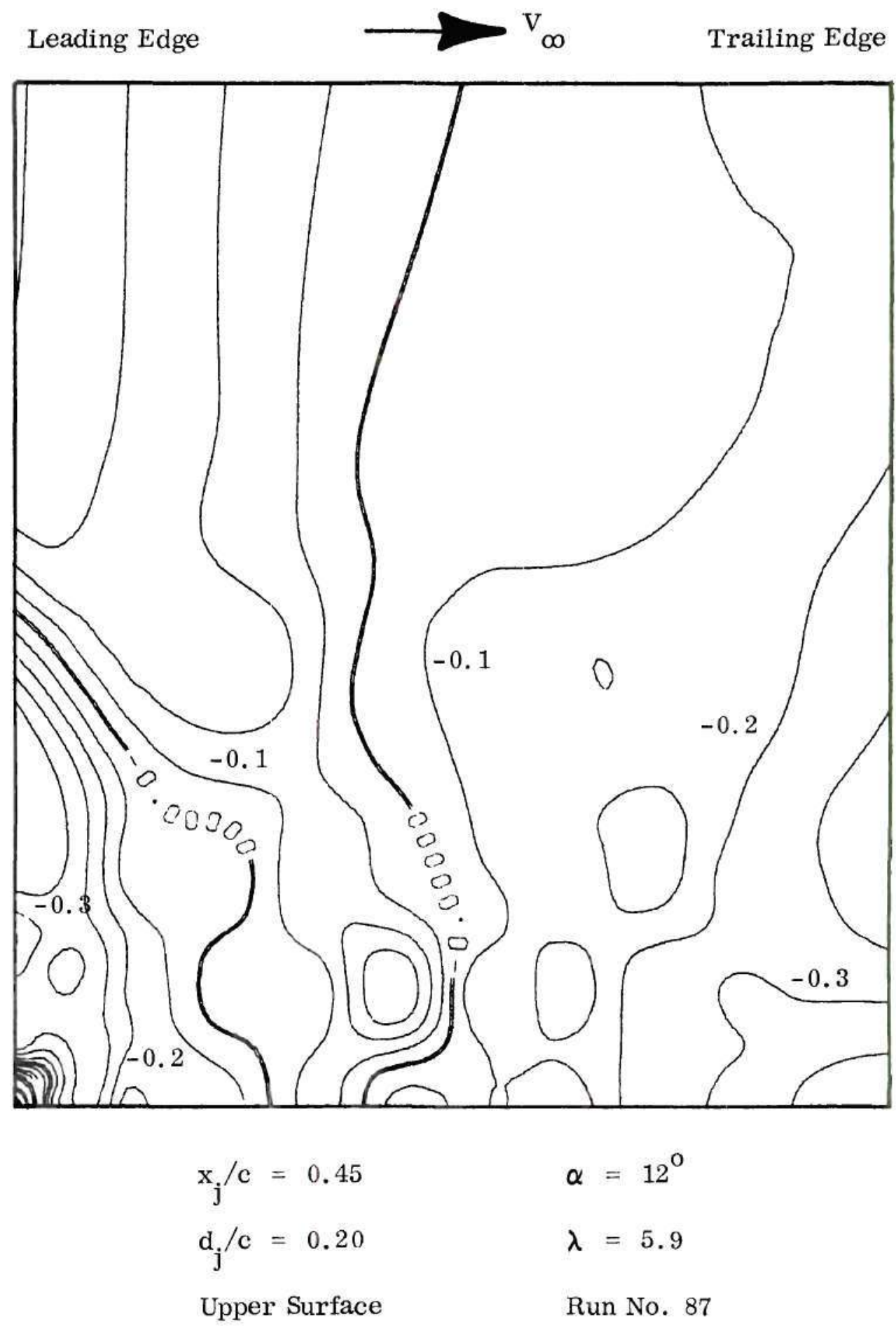

Figure C-4x. Interference Surface Pressure Distribution. 


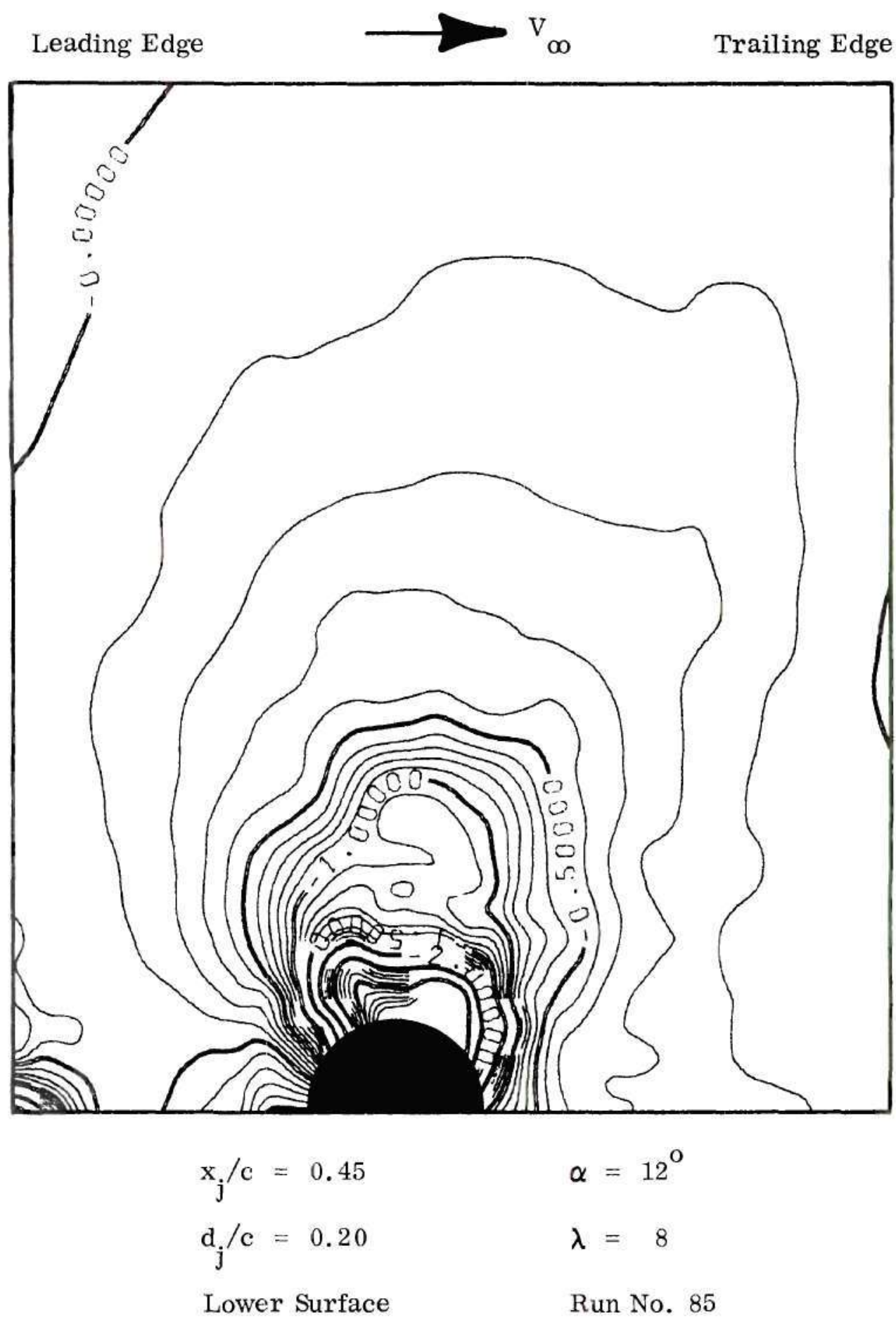

Figure C-4y。 Interference Surface Pressure Distribution. 


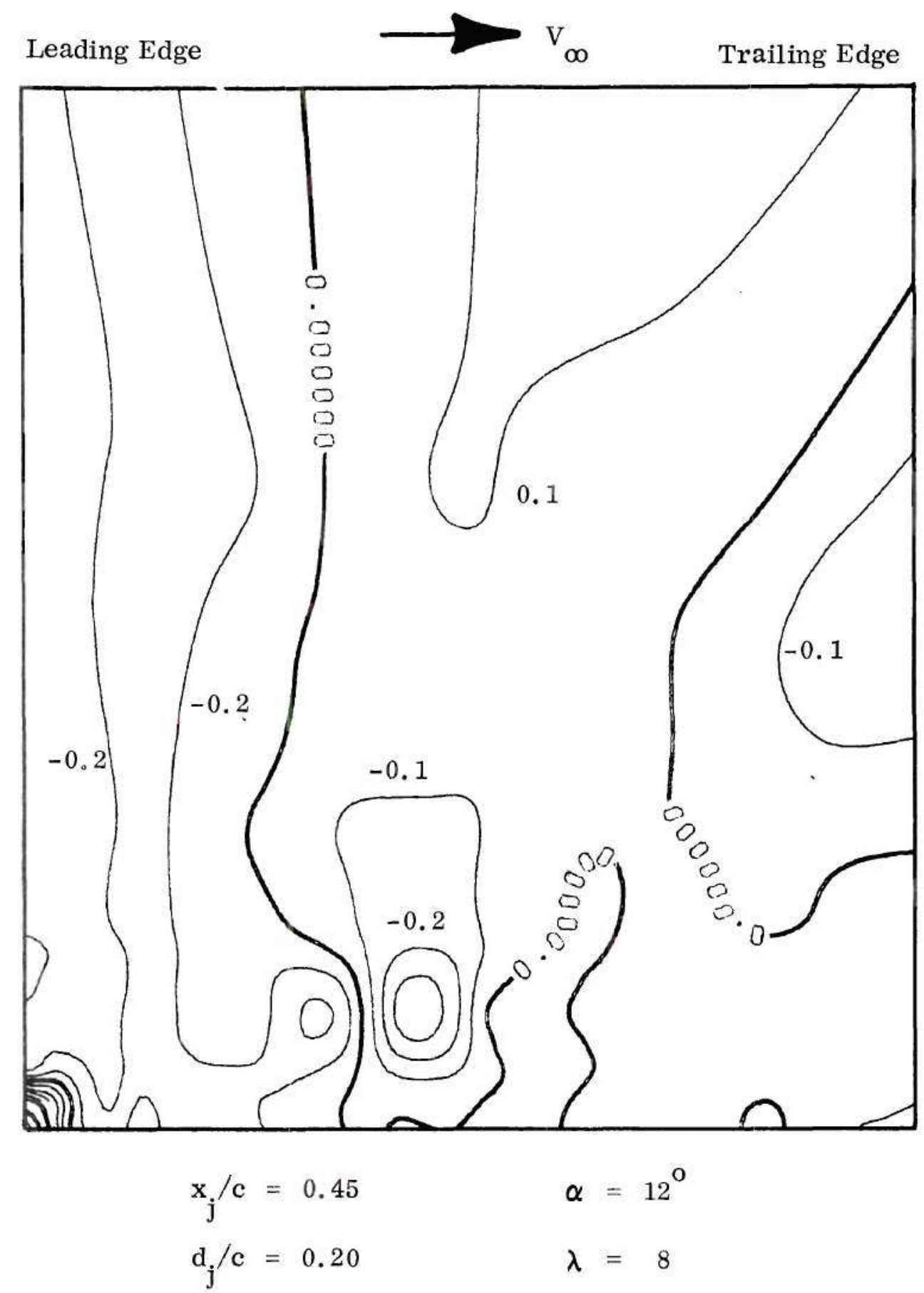

Upper Surface Run No. 85

Figure $\mathrm{C}-4 \mathrm{z}$. Interference Surface Pressure Distribution。 
APPENDIX D

INTERFERENCE LIFT, DRAG, AND PITCHING MOMENT COEFFICIENTS

This appendix contains a summary of the interference lift, drag, and pitching moment data collected in this investigation. The coefficients were computed in the following manner:

$$
\begin{aligned}
& \frac{\Delta L}{T}=\frac{L_{\text {jet-on }}-L_{\text {jet-off }}}{T} \\
& \frac{\Delta D}{T}=\frac{D_{\text {jet-on }}-D_{\text {jet-off }}}{T} \\
& \frac{\Delta M}{T d_{j}}=\frac{P M \text { jet-on }^{-P M_{\text {jet-off }}}}{T d_{j}}
\end{aligned}
$$

These results are presented uncorrected for angle of attack and for jet exit location. This was done so that the adverse effect attributed directly to jet thrust for drag at a nonzero angle of attack and the favorable effect of a jet exit location aft of the quarter chord would be reflected in the data.

All force data was reduced with the electronic computer. The incremental forces and moments were determined by

$$
\begin{aligned}
& \Delta L=L_{\text {jet-on }}-q_{\infty} S\left[C_{L}\right]_{\text {jet-off }} \\
& \Delta D=D_{\text {jet-on }}-q_{\infty} S\left[C_{D}\right]_{\text {jet-off }}
\end{aligned}
$$




$$
\Delta \mathrm{M}=(\mathrm{PM})_{\text {jet-on }}-\mathrm{q}_{\infty} \mathrm{S}\left[\mathrm{C}_{\mathrm{M}}\right]_{\text {jet-off }}{ }^{\mathrm{c}}
$$

where the value for $\mathrm{q}_{\infty}$ is that at which the jet-on measurements were made.

Recall from Chapter IV, however, that the jet-off aerodynamic coefficients were a function of tunnel freestream velocity. Hence, the jet-off coefficients that corresponded to the $\mathrm{q}_{\infty}$ at which the jet-on measurements were made were determined by a second order Aitken interpolation of the known functional relationship between the aerodynamic coefficients and freestream velocity. (For data reduction purposes, the freestream dynamic pressure was used as the independent variable in this functional relationship.) The jet thrust, as computed from the strain gage bridge readouts using the matrix inversion described in Appendix B, was then used for the calculation of the interference force and moment coefficients. A sample computer printout of the interference force and moment data is presented in Table D-1.

The reader is reminded that in the form presented a zero interference lift corresponds to $\frac{\Delta L}{T}=1.0$ (i.e. for a lift loss, $\frac{\Delta L}{T}<1.0$ ). Conversely, zero interference drag and pitching moment yield $\frac{\Delta \mathrm{D}}{\mathrm{T}}=0.0$ and $\frac{\Delta \mathrm{M}}{\mathrm{Td}_{\mathrm{j}}}=0.0$ respectively. As noted in Chapter III, the maximum error in $\frac{\Delta \mathrm{L}}{\mathrm{T}}$ is \pm 4.0 percent for $\lambda=8$, \pm 10.0 percent for $\lambda=4$, and \pm 20 percent for $\lambda=2$. The interference drag and pitching moment coefficients should not be interpreted quantitatively.

The interference lift coefficients are presented in Figures D-1 for the various geometries tested. The interference drag coefficients are presented in Figures D-2 and the interference pitching moment coefficients in Figure D-3. 
Table D-1. Sample Computer Printout of Interference

Force and Moment Data

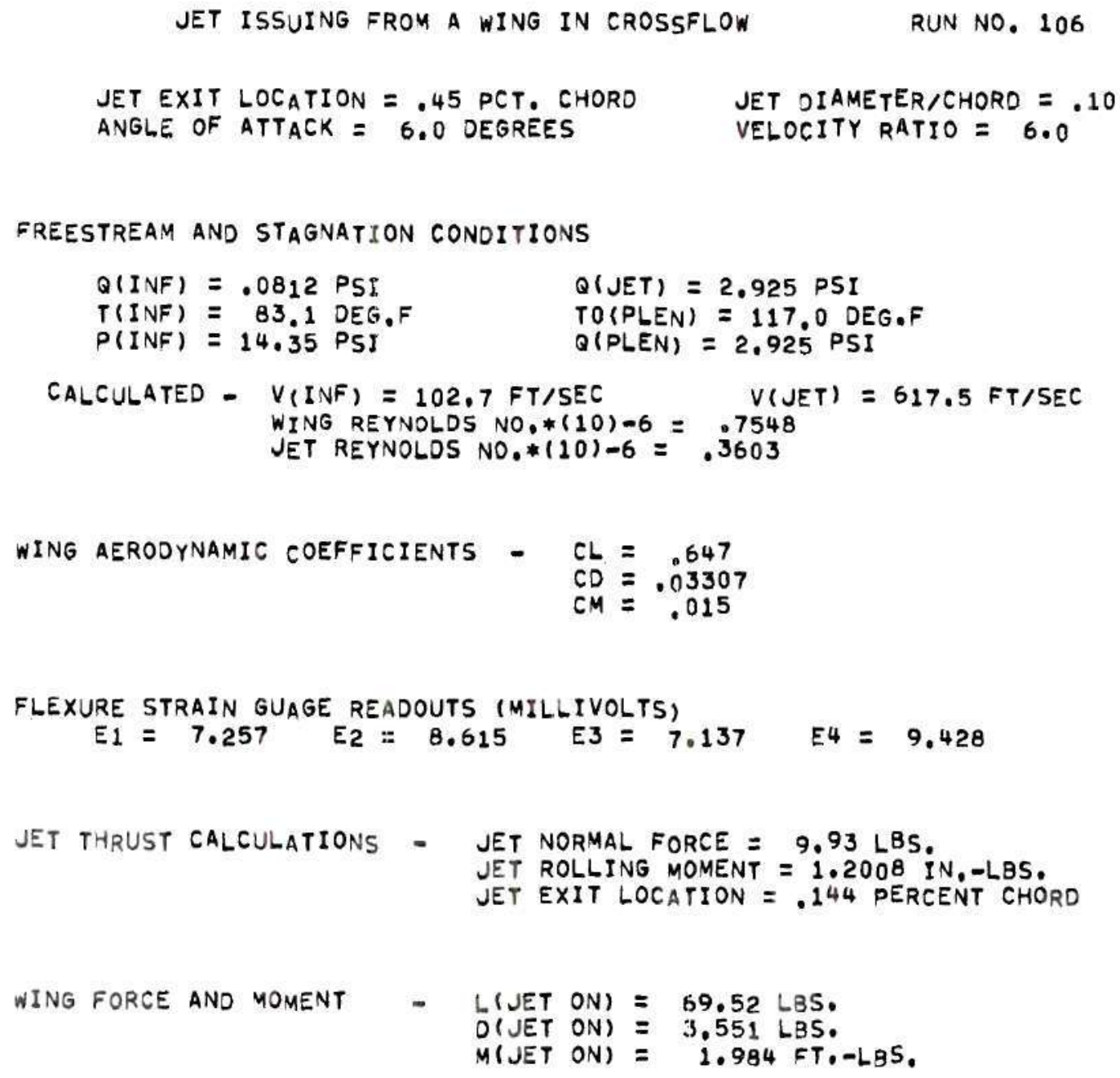

WING FORCE AND MOMENT - LIJET ON) $=69.52$ LBS. O(JET ON $=3.551$ LBS. $M(J E T$ ON $)=1.984$ FT. $-L B S$.

INTERFERENCE FORCE AND MOMENT COEFFICIENTS

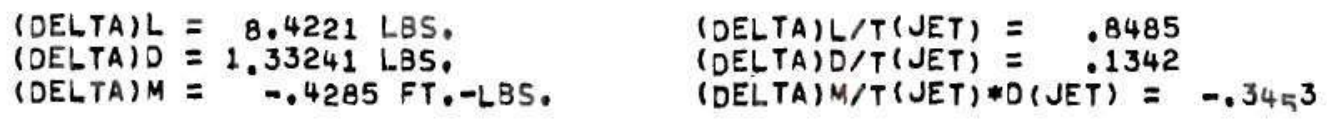




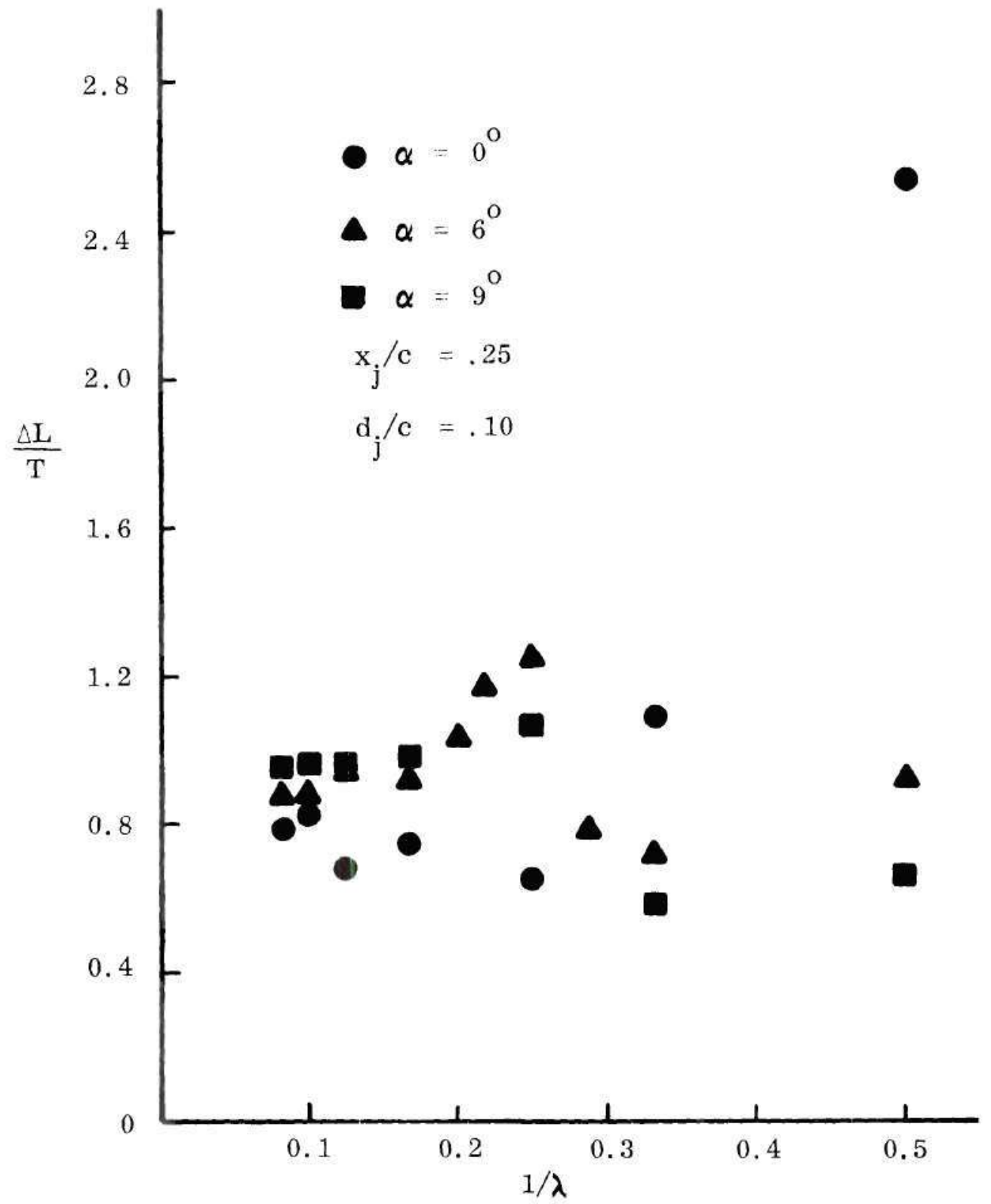

Figure D-1a. Interference Lift Coefficients. 


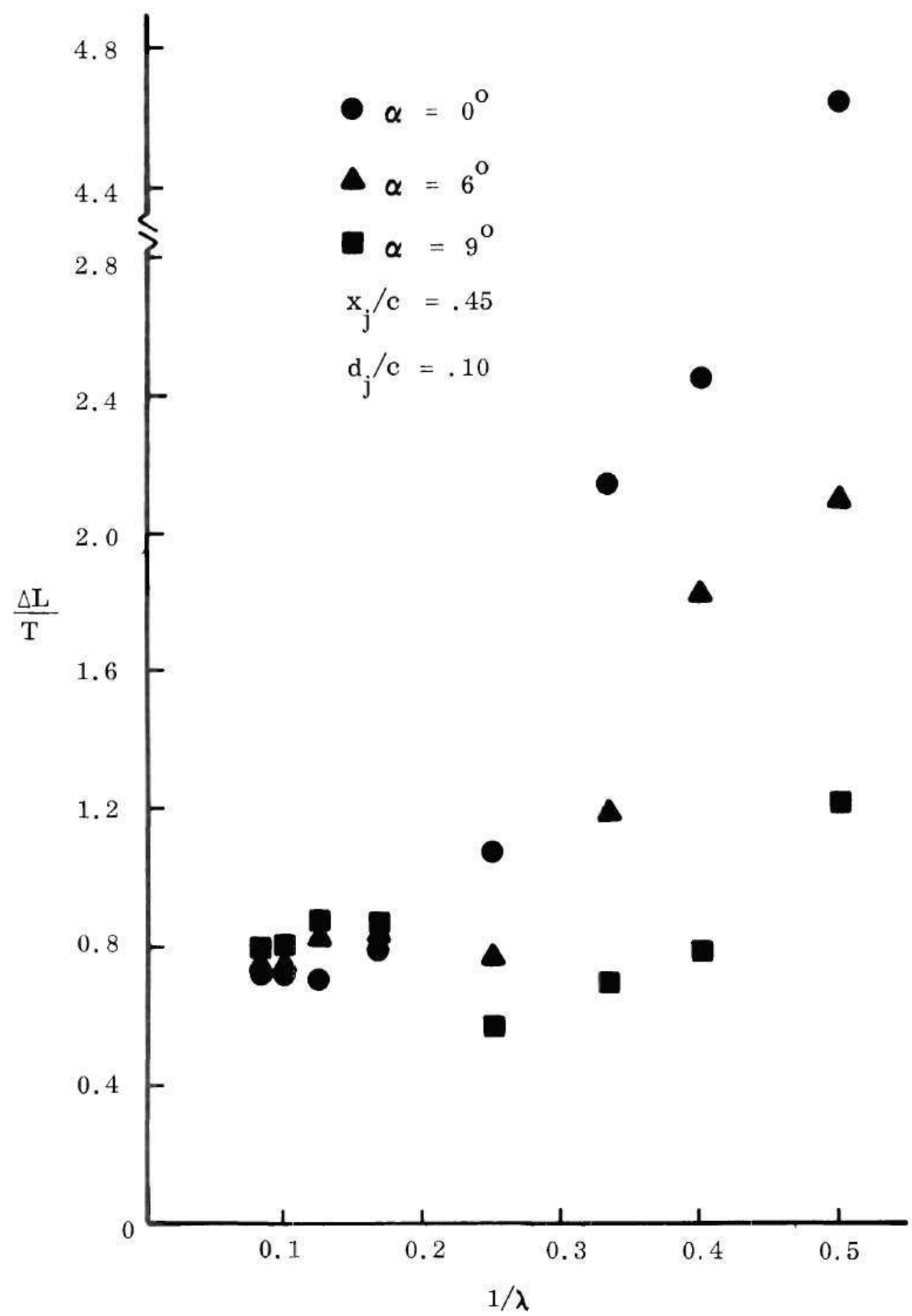

Figure D-1b. Interference Lift Coefficients. 


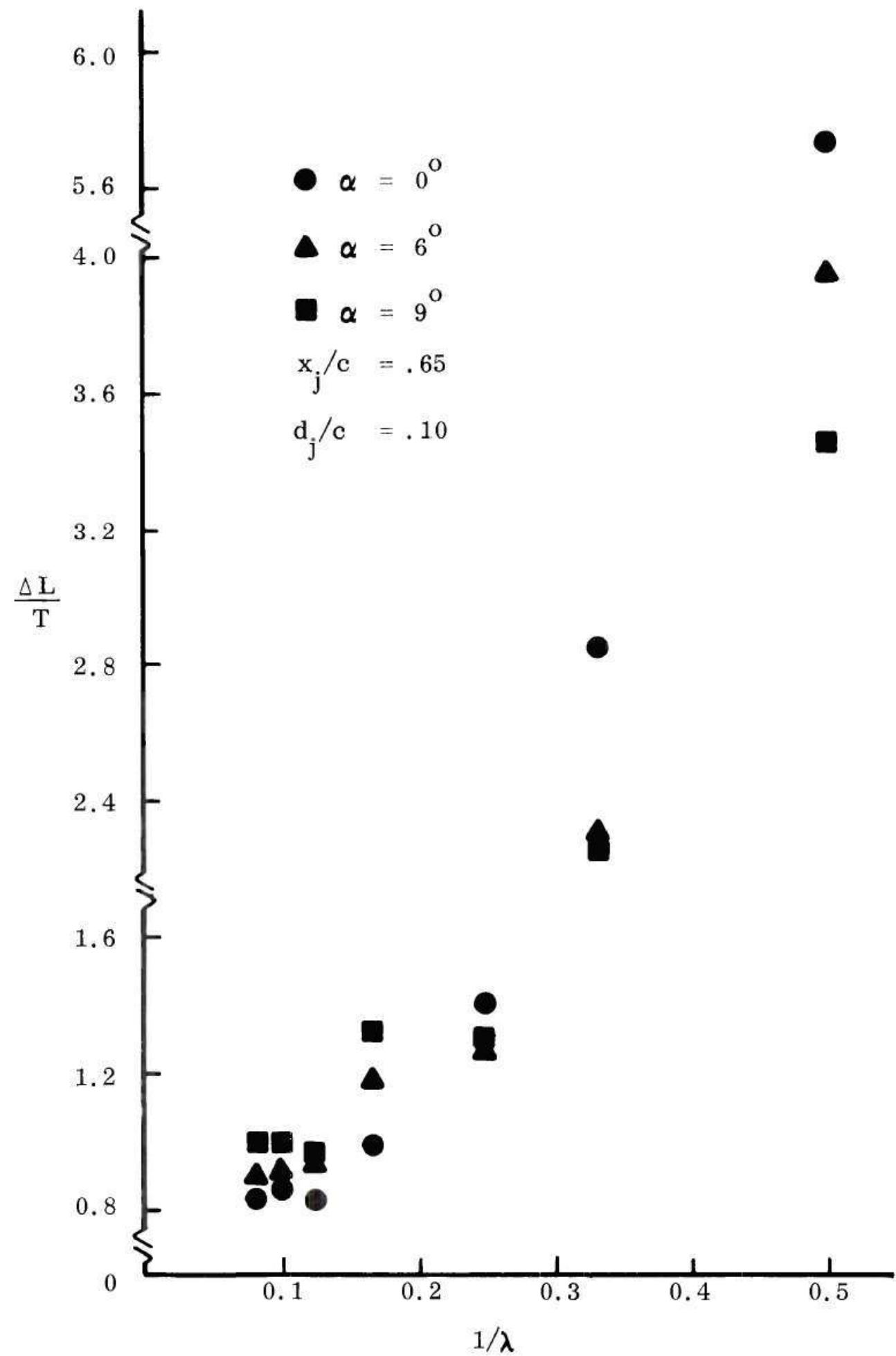

Figure D-1C. Interference Lift Coefficients. 


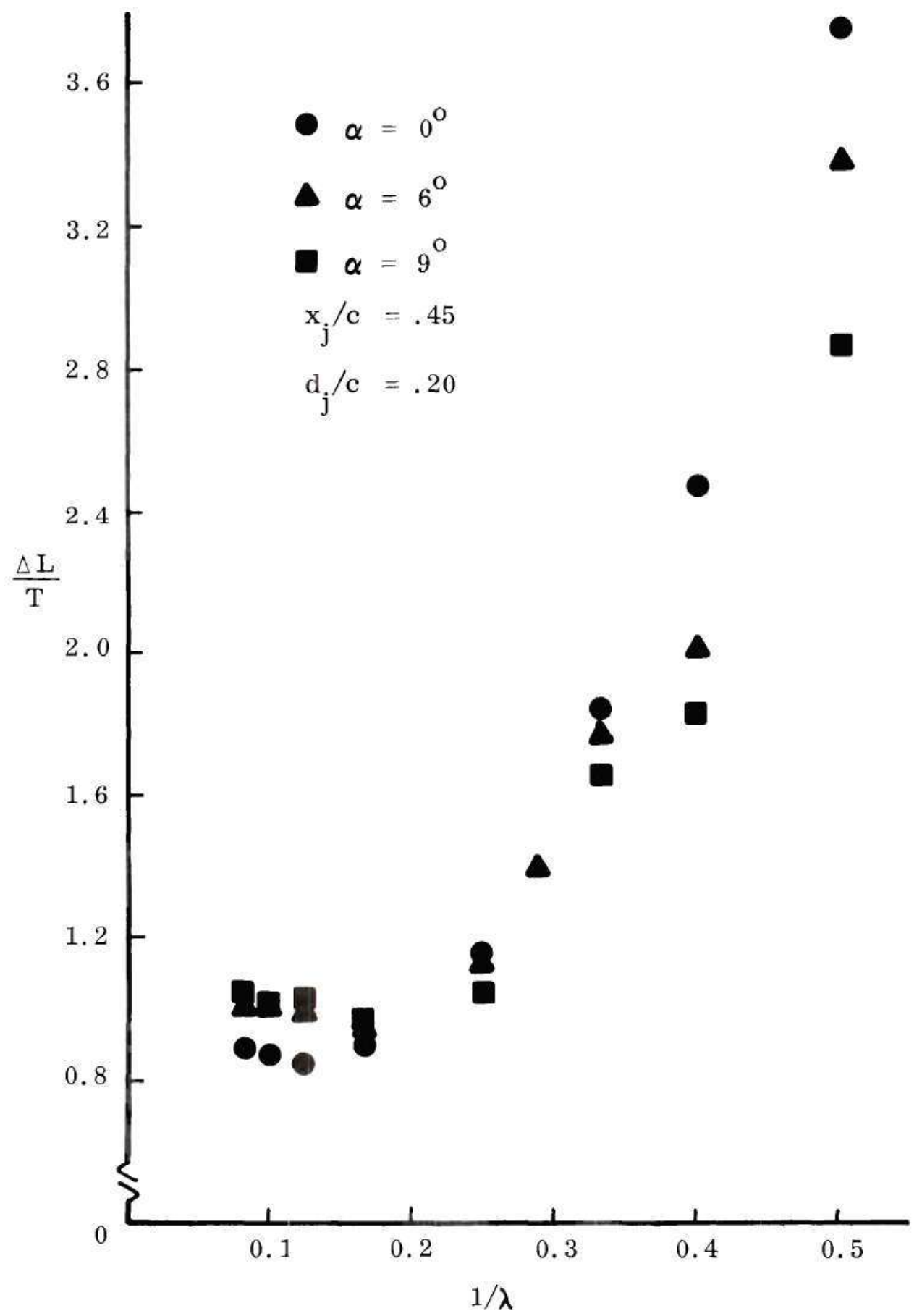

Figure D-1d. Interference Lift Coefficients. 


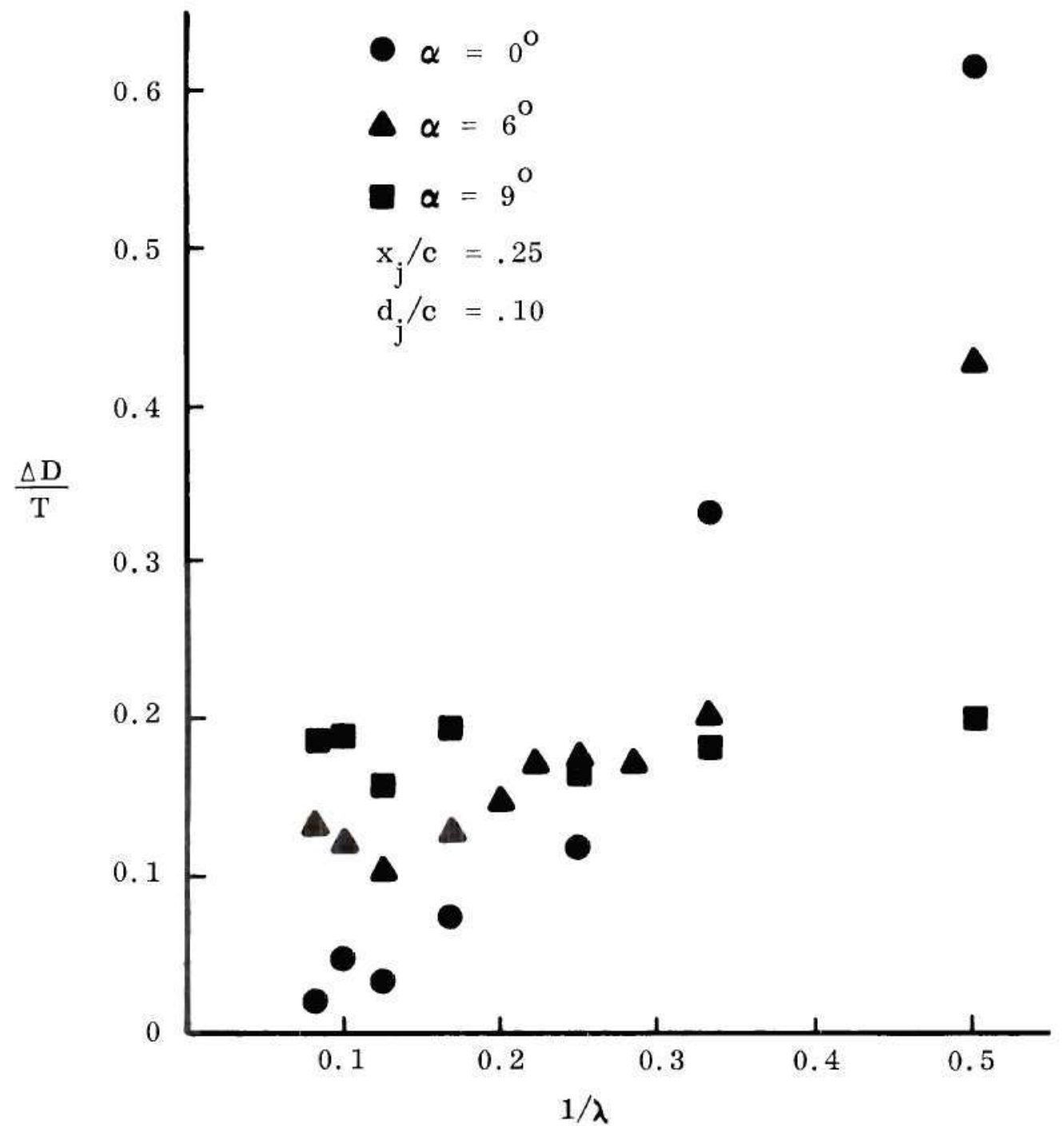

Figure D-2a. Interference Drag Coefficients. 


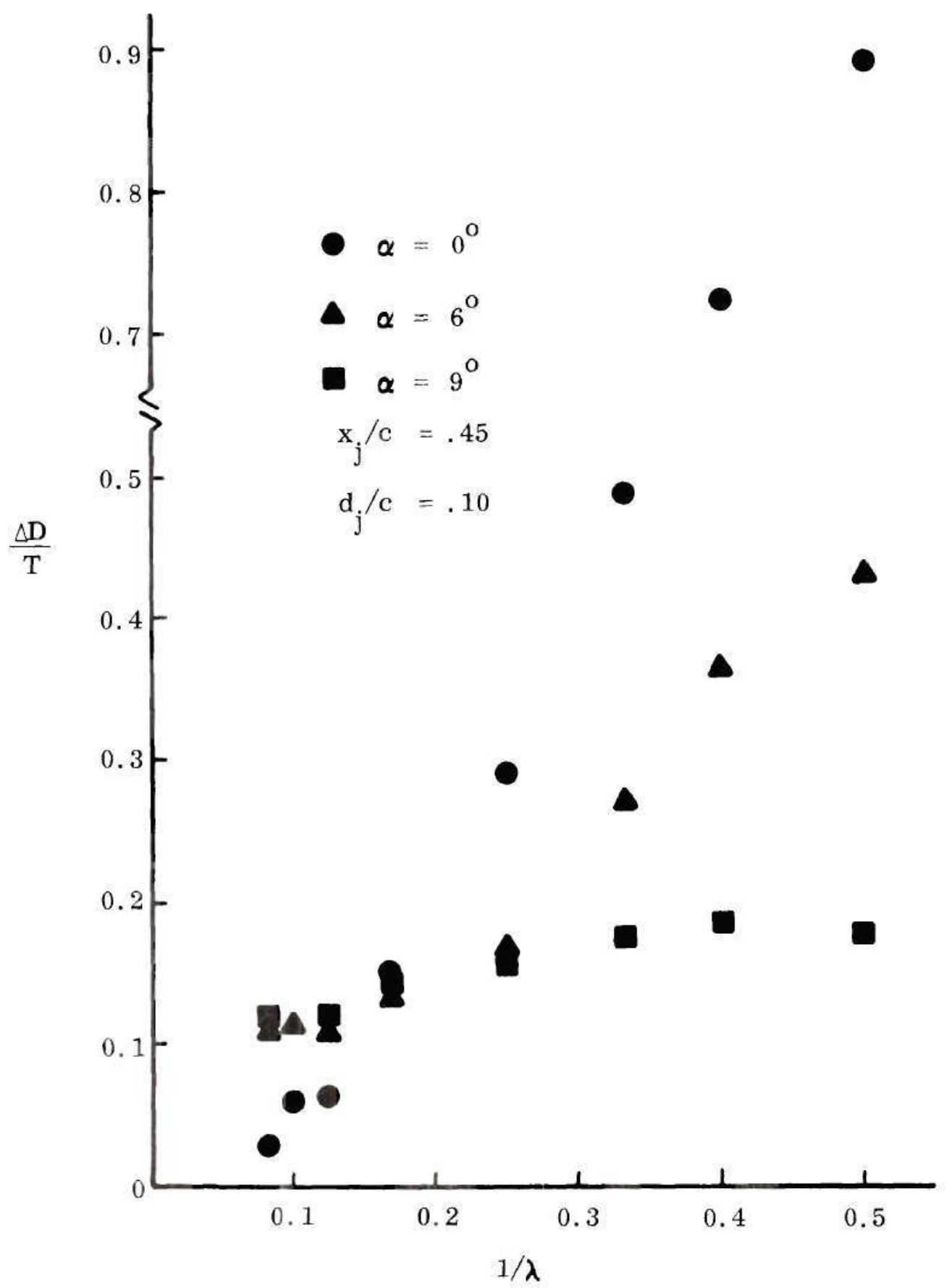

Figure D-2b. Interference Drag Coefficients. 


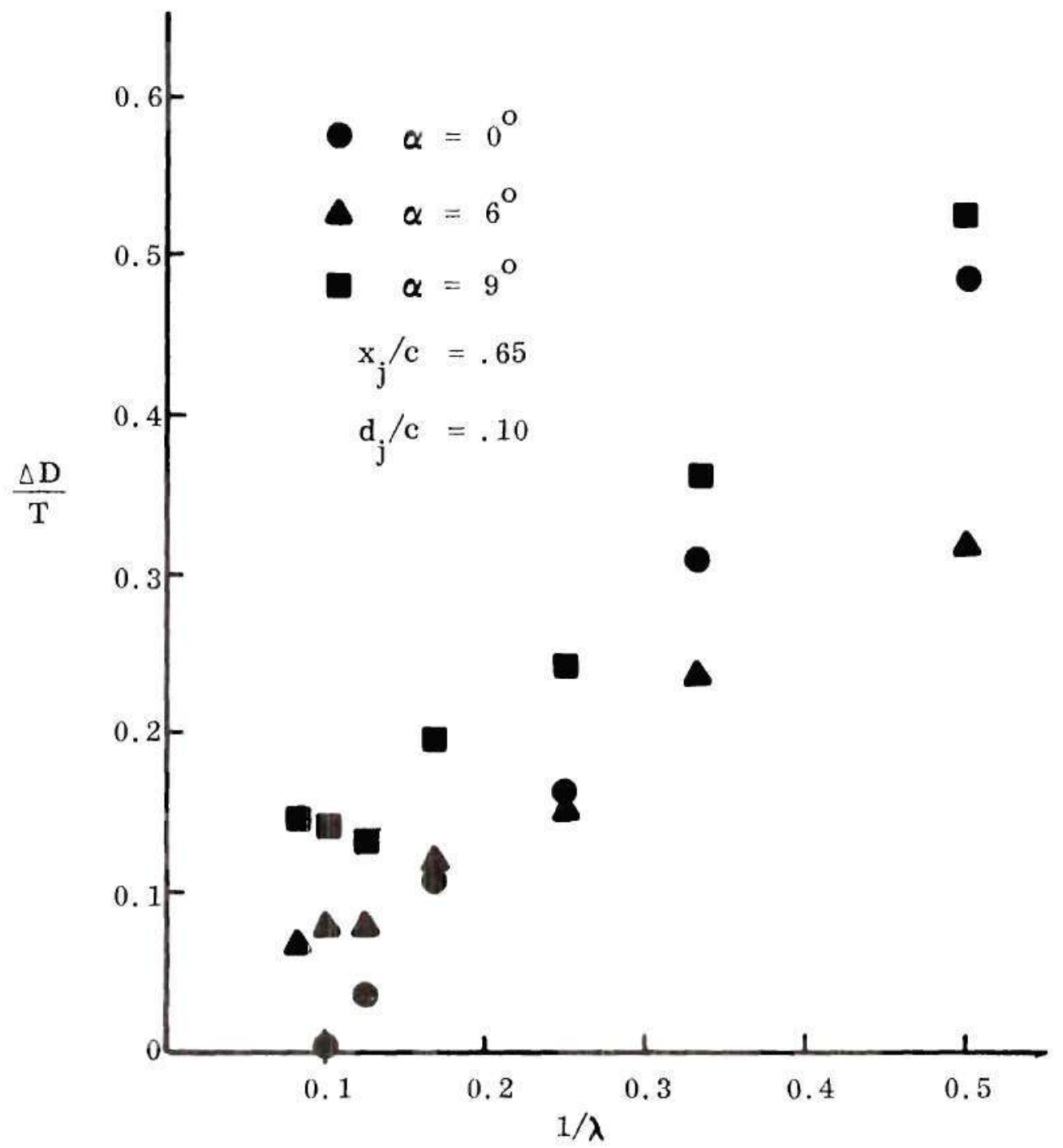

Figure D-2c. Interference Drag Coefficients. 


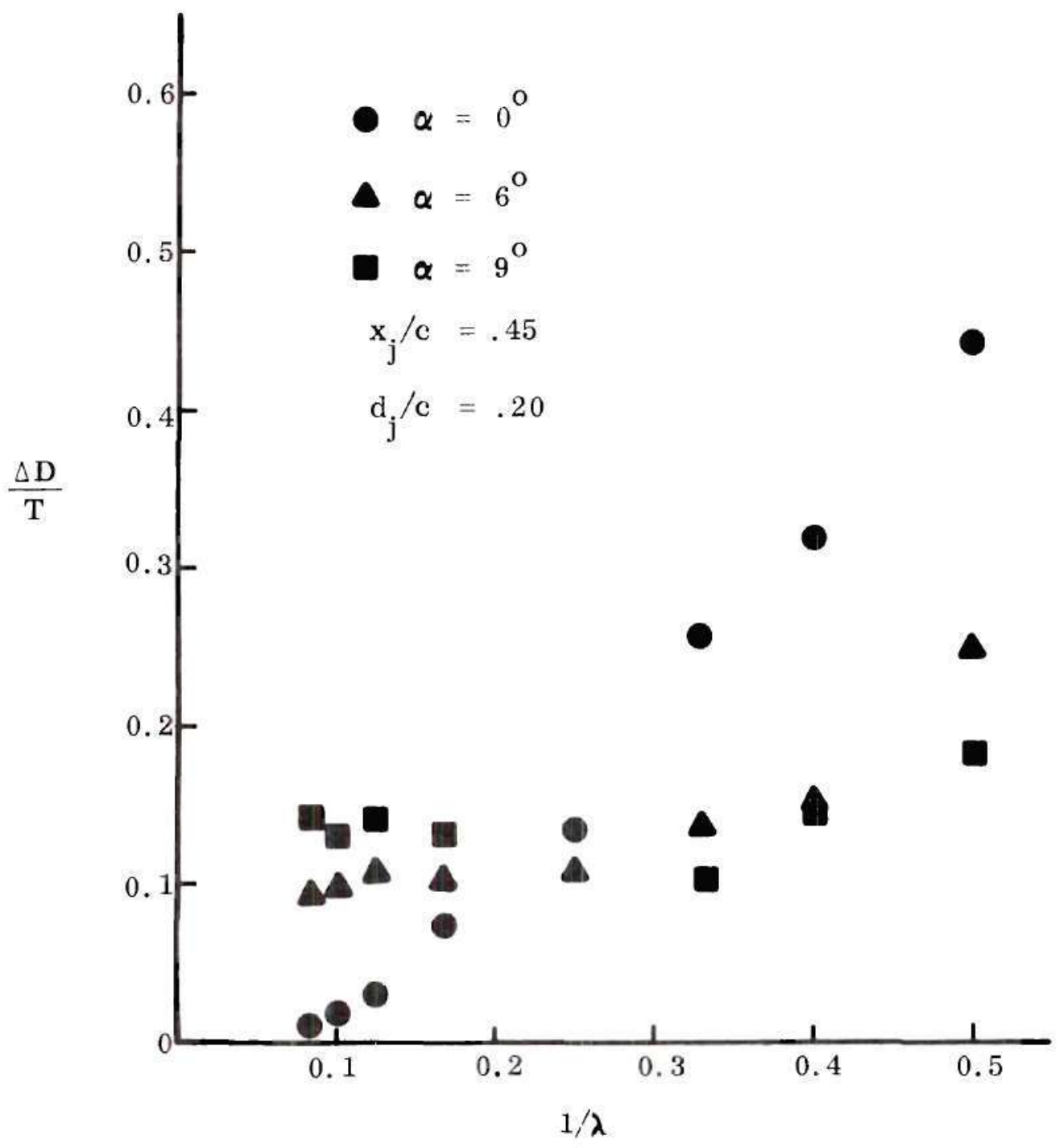

Figure D-2d. Interference Drag Coefficients. 


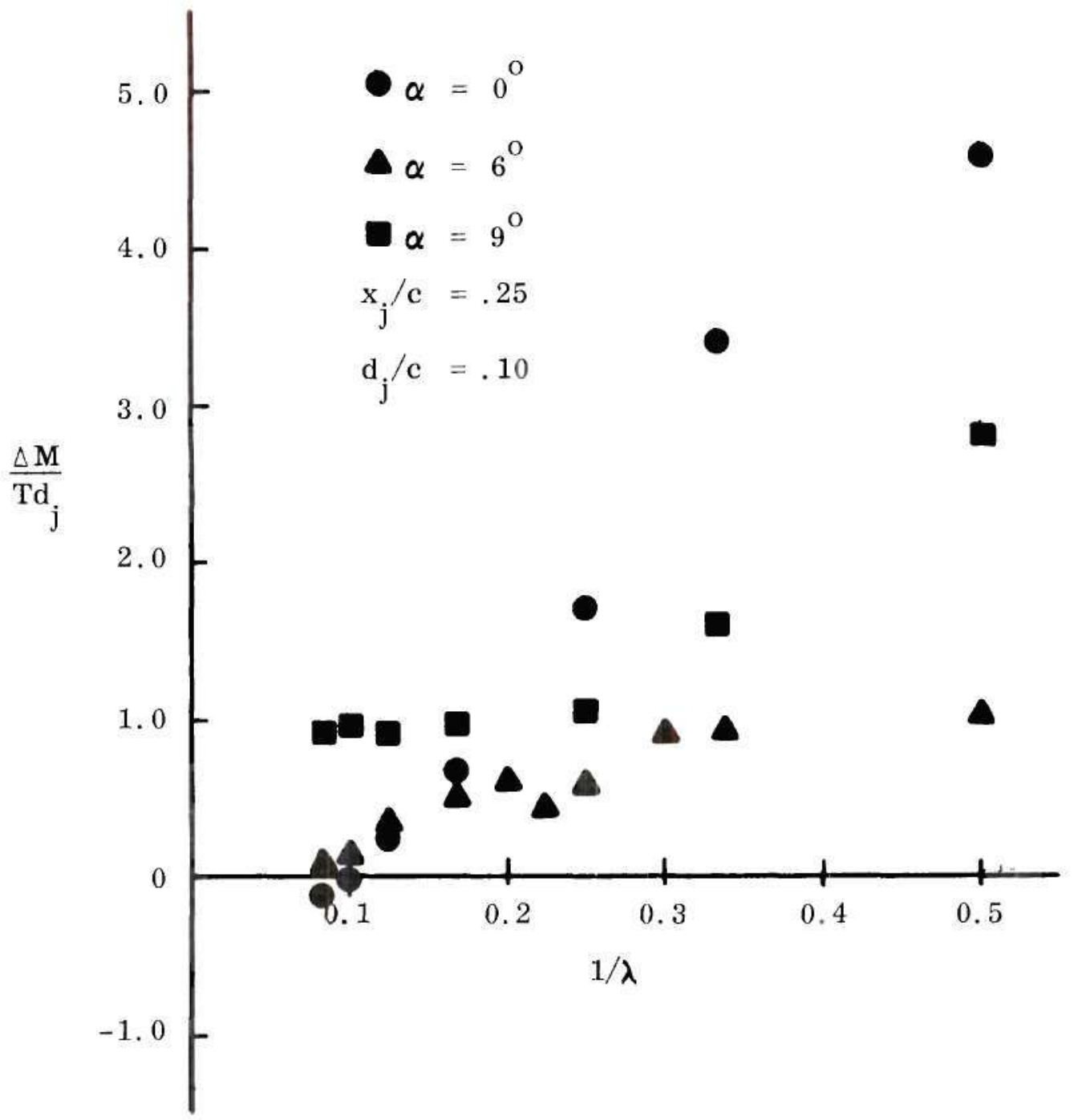

Figure D-3a. Interference Pitching Moment Coefficients. 


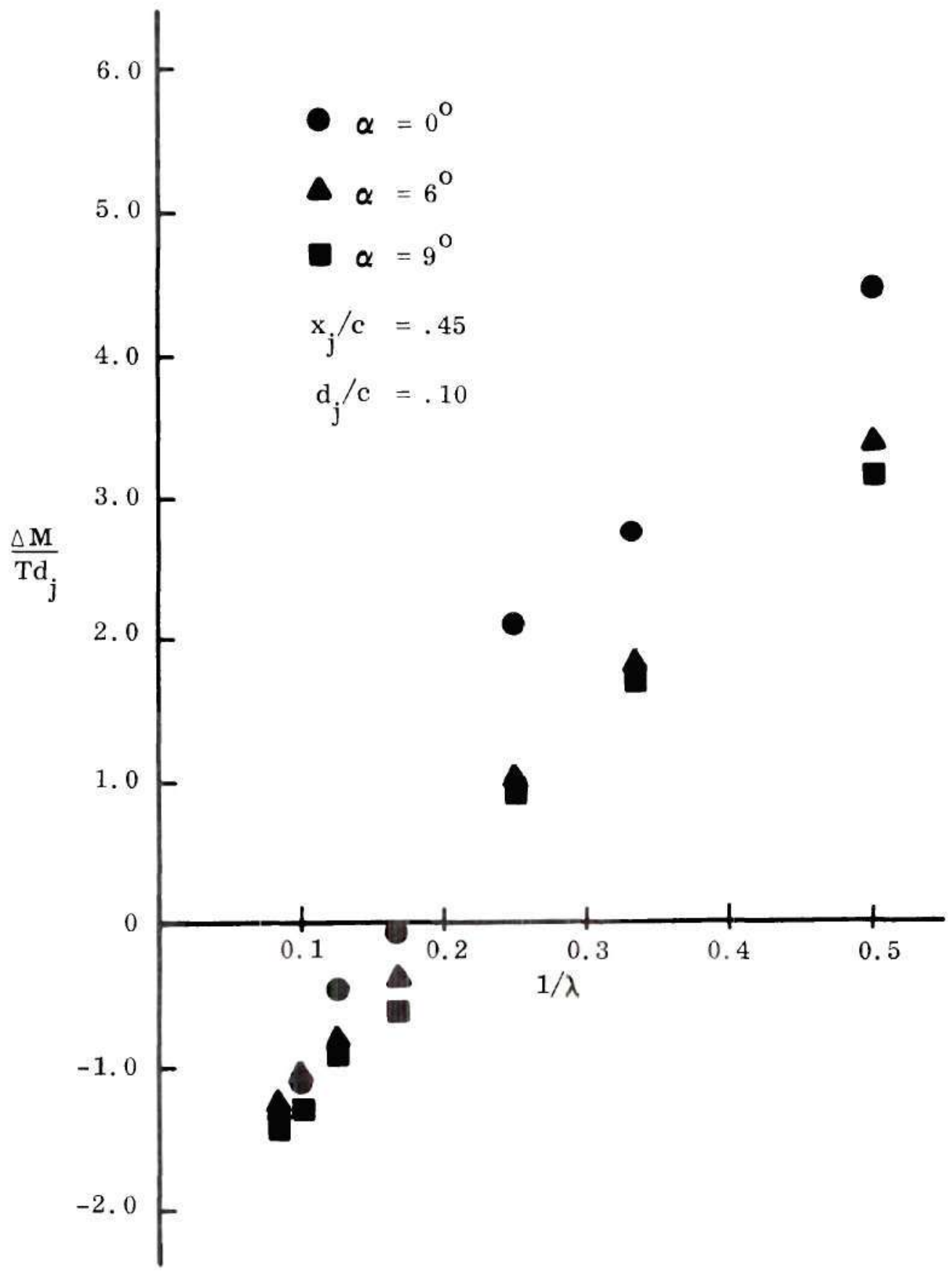

Figure D-3b. Interference Pitching Moment Coefficients. 


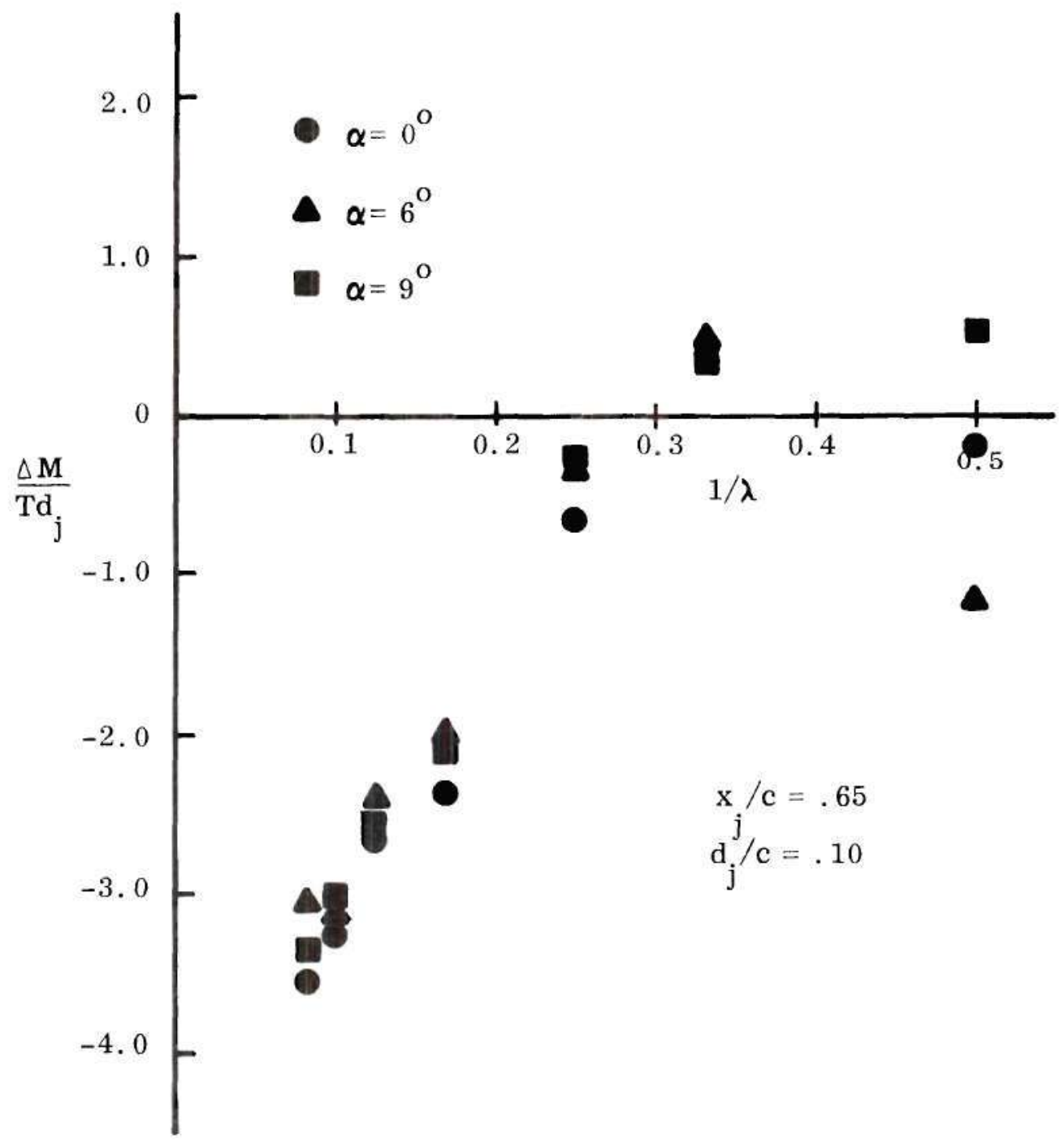

Figure D-3c. Interference Pitching Moment Coefficients. 


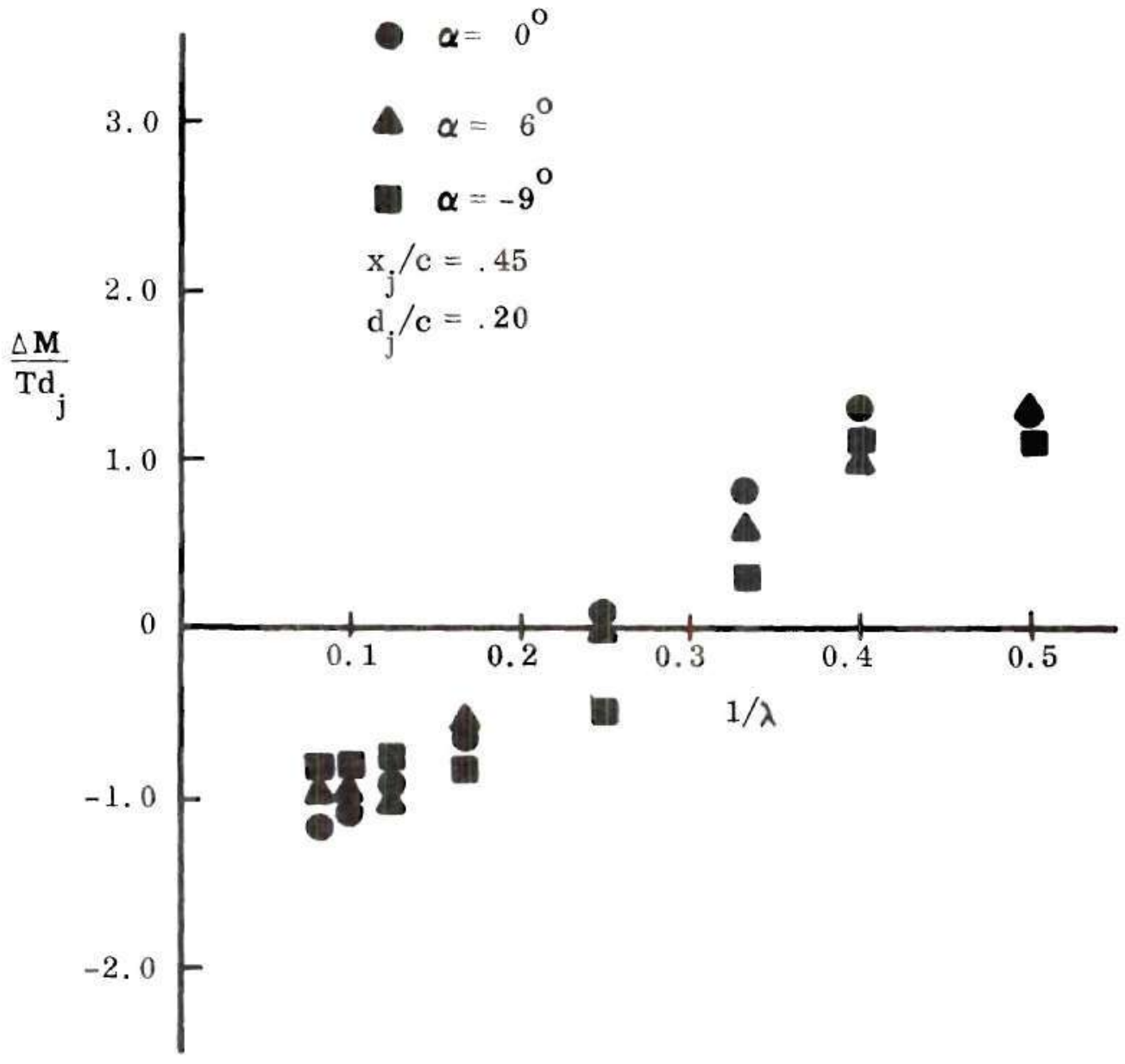

Figure D-3d. Interference Pitching Moment Coefficients.

$$
x_{j} / c=.45, d_{j} / c=.20
$$




\section{LITERATURE CITED}

1. Vogler, R. D., "Ground Effects on Single and Multiple Jet Models at Transition Speeds Over Stationary and Moving Ground Planes," TN D-3213, 1966, NASA.

2. Vogler, R. D., "Interference Effects of Single and Multiple Round or Slotted Jets on a VTOL in Transition," TN D-2380, Aug. 1964, NASA.

3. Spreeman, K. P., "Induced Interference Effects on Jet and Buried-Fan VTOL Configurations in Transition," TN D-731, Mar. 1961, NASA.

4. Otis, J. H., "Induced Interference Effects on a Four-Jet VTOL Configuration with Various Wing Planforms in the Transitional Speed Range," TN D-1400, Sept. 1962, NASA.

5. Margason, R. J. and Gentry, G. L., "Aerodynamic Characteristics of a Five-Jet VTOL Configuration in the Transition Speed Range," TN D-4812, Oct. 1968, NASA.

6. Margason, R. J., "Jet-Induced Effects in Transition Flight," SP-116, April 1966, NASA.

7. Margason, R. J., "Review of Propulsion Induced Effects on Aerodynamics of Jet/STOL Aircraft," TN D-5617, Feb. 1970, NASA.

8. Kirk, J. V., Hickey, D. H., and Hall, L. P., "Aerodynamic Characteristics of a Full-Scale Fan-in-Wing Model Including Results in Ground Effects with Nose-Fan Pitch Control," TN D-2368, July 1964, NASA.

9. Kirk, J. V., Hodder, B. K., and Hall, L. P., "Large Scale Wind Tunnel Tests of a V/STOL Transport Model with Wing-Mounted Lift Fans and Fuselage Mounted Lift-Cruise Engines for Propulsion," TN D-4233, 1967, NASA.

10. Trebble, W. J. G. and Williams, J., "Exploratory Wind-Tunnel Investigations on a Bluff Body Containing a Lifting Fan," C.P. No. 597, Apr. 1961, British Royal Aircraft Establishment.

11. Hickey, D. and Hall, L., "Aerodynamic Characteristics of a Large-Scale Model with Two High Disc-Loading Fans Mounted in the Wing," TN D-1650, Feb. 1963, NASA. 
12. Maki, R. L. and Hickey, D. H., "Aerodynamics of a Fan-in-Fuselage Model," TN D-789, 1961, NASA.

13. Davenport, E. E. and Kuhn, R. E., "Wind Tunnel Wall Effects and Scale Effects on a VTOL Configuration with a Fan Mounted in the Fuselage," TN D-2560, 1965, NASA.

14. Aoyagi, K. and Hickey, D. H., "Aerodynamic Characteristics of a Large Scale Model with a High Disk-Loading Fan Mounted in the Fuselage," TN D-775, 1961, NASA.

15. Mosher, D. K., "An Experimental Investigation of a Turbulent Jet in a Crossflow," PhD Thesis, December 1970, Georgia Institute of Technology.

16. Keffer, J. F. and Baines, W. D., "The Round Turbulent Jet in a Cross Wind," Journal of Fluid Mechanics, Vol. 15 Part 4, 1963, pp. 481-496.

17. Jordinson, R., "Flow in a Jet Directed Normal to the Wind," R \& M 3074, October 1956, British Aero. Res. Council.

18. Gelb, G. H. and Martin, W. A., "An Experimental Investigation of the Flow Field about a Subsonic Jet Exhausting into a Quiescent and a Low Velocity Air Stream," Canadian Aeronautics and Space Journal, Vol. 12, No. 8, October 1966, pp. 333-342.

19. Bradbury, L. J. S. and Wood, M. N., "The Static Pressure Distribution Around a Circular Jet Exhausting Normally from a Plane Wall into an Airstream," Tech. Note No. Aero 2978, Aug. 1964, British Royal Aircraft Establishment.

20. Peake, D. J., "The Pressures on a Surface Surrounding a Jet Issuing Normal to a Mainstream," Aeronautical Report LR-410, Nov. 1964, National Research Council of Canada, Ottawa, Can.

21. Wooler, P. T., "On the Flow Past a Circular Jet Exhausting at Right Angles from a Flat Plate or a Wing," Journal of the Royal Aeronautical Society, Vol. 71, March 1967, pp. 216-218.

22. Margason, R. J., "Analysis of a Jet in a Subsonic Crosswind," SP-218, Sept. 1969, NASA.

23. Williams, J. and Wood, M. N., "Aerodynamic Interference Effects with JetLift V/STOL Aircraft under Static and Forward-Speed Conditions," Zeitschrift fur Flugwissenshaften, Vol. 15, No. 7, July 1967, pp. 237-256. 
24. Skifstad, J. G., "Aerodynamics of Jets Pertinent to VTOL Aircraft," Journal of Aircraft, Vol. 7, No. 3, May-June 1970, pp. 193-204.

25. Hickey, D. H. and Ellis, D. R., "Wind Tunnel Tests of a Semispan Wing with a Fan Rotating in the Plane of the Wing," TN D-88, Oct. 1959, NASA.

26. Gregory, N., Raymer, W. G., and Love, E. M., "Wind Tunnel Tests of a Wing Fitted with a Single Lifting Fan," R \& M 3457, 1967, British Aero. Res. Council.

27. Duvivier, J. F. and McCallum, R. B., "Investigation of Tilting Duct and Fan-Wing in Transition Flight," Tech. Rpt. 90-1, 1964, Aeroelastic and Structures Res. Lab., Massachusetts Institute of Technology.

28. Carter, A., "Effects of Jet-Exhaust Location on the Longitudinal Aerodynamic Characteristics of a Jet V/STOL Model," TN D-5333, July 1969, NASA.

29. Wooler, P. T., Burghart, G. H. and Gallagher, J. T., "The Pressure Distribution on a Rectangular Wing with a Jet Exhausting Normally into an Airstream," Journal of Aircraft, Vol. 4, No. 6, Nov.-Dec. 1967, pp.537543.

30. Tyler, R. A. and Williamson, R. G., "Observations of Tunnel Flow Separation Induced by an Impinging Jet," Aero. Rpt. LR-537, April 1970, NRC of Canada.

31. Wijker, H., "Survey of Transition Point Measurements at the N. L. L., Mainly for Two-Dimensional Flow Over a NACA 0018 Profile," Report A. 1269 , N. L. L. of Holland.

32. Williams, J., Butler, S.F.J., and Wood, M. N., "The Aerodynamics of Jet-Flaps," R \& M 3304, Jan. 1961, British Aero. Res. Council.

33. Dimmock, N. A., "An Experimental Introduction to the Jet-Flap," C.P. No. 344, 1957, British Aero. Res. Council.

34. Alexander, A. J., and Williams, J., "Wind Tunnel Experiments on a Rectangular-Wing Jet-Flap Model of Aspect - Ratio 6," R \& M 3329, June 1961, British Aero. Res. Council.

35. Wasson, H. and Kao, H., Unpublished Work Performed at the Northrop Corporation, Aircraft Division.

36. Gentry, G. L., and Margason, R. J., "Jet-Induced Lift Losses on VTOL Configurations Hovering in and out of Ground Effect," TN D-3166, Feb. 1966, NASA.

37. Whipkey, R., Unpublished Memorandum, Lockheed-Georgia Co. 


\section{OTHER REFERENCES}

Garner, J. E., "A Review of Jet Efflux Studies Applicable to V/STOL Aircraft," AEDC-TR-67-163, September 1967.

Jacobs, E. N., "Tests of Six Symmetrical Airfoils in the Variable Density Wind Tunnel," TN No. 385, July 1931, NACA.

McCormick, B. W., Aerodynamics of V/STOL Flight, Academic Press, New York, New York, 1967.

Mikolowsky, W. T., "A Method for Computing the Viscous Compressible Pressure Distribution on a Two-Dimensional Airfoil," Unpublished Special Problem for $\mathrm{AE} \mathrm{604,} \mathrm{June} \mathrm{1970,} \mathrm{Georgia} \mathrm{Institute} \mathrm{of} \mathrm{Technology.}$

Pope, A. and Harper, J. J., Low-Speed Wind Tunnel Testing, John Wiley and Sons, Inc., New York, New York, 1966.

Strand, T., "A Slanted Round Jet at Low Forward Speeds," J. of Aircraft, Vol. 8, No. 4, April 1971, pp. 278 279.

Ward, K. E., "The Effect of Small Variation in Profile of Airfoils," TN No. 361, January 1931, NACA.

Wickens, R. H., "The Trailing Vortex Wake Downwind of an External Flow JetFlap," Canadian Aero. and Space J., Vol. 18, No. 3, March 1972, pp. 67-68.

Williams, J. andButler, S. F.J., "Recent Developments in Low Speed Wind-Tunnel Techniques for V/STOL and High-Lift Model Testing," Zeitschrift fur Flugwissenshaften, Vol. 13, No. 3, March 1965, pp. 73-89. 


\section{VITA}

William T. Mikolowsky, the oldest son of Mrs. Emma Mikolowsky and the late Mr. George E. Mikolowsky, was born in Uniontown, Pennsylvania on July 5, 1944. He attended elementary and secondary schools in Masontown, Pennsylvania and graduated from Albert Gallatin Senior High School in May, 1962 as Valedictorian of his class. Mr. Mikolowsky entered the Georgia Institute of Technology in September, 1963 and received his Bachelor of Aerospace Engineering, with Highest Honors in June, 1968. As an undergraduate, he was a member of Phi Kappa Phi, Tau Beta Pi, Sigma Gamma Tau, and Phi Eta Sigma honorary societies.

Mr. Mikolowsky was employed as an Engineering Co-op Trainee at the Lockheed-Georgia Company in Marietta, Georgia from January, 1964 through September, 1967. Upon graduation from Georgia Tech, he joined the LockheedGeorgia Co. as an Associate Aircraft Engineer in the Aerodynamic Technology Department. His prime work area was the development of methods for predicting the aerodynamic characteristics of two-dimensional airfoils including the effects of viscosity and compressibility.

In September, 1968, Mr. Mikolowsky returned to the Georgia Institute of Technology and entered the doctoral program in Aerospace Engineering. He was awarded a National Defense Education Act, Title IV Fellowship and also served in various capacities as graduate research assistant and a graduate teaching assistant in mathematics. Mr. Mikolowsky received his Master of Science in Aerospace 
Engineering in June 1970. He was a student member of the American Institute of Aeronautics and Astronautics and was active in Student Government, serving as Executive Vice-President of the Graduate Student Senate and as Chairman of the Georgia Tech Student Traffic Court. In 1971, he was listed in Who's Who Among Students in American Universities and Colleges and in 1972 was elected to the Omicron Delta Kappa leadership honorary. He is also a member of the Society of the Sigma Xi.

On July 18, 1964, in Atlanta, Georgia, Mr. Mikolowsky married the former Barbara Ann Kozel of Masontown, Pennsylvania. They have a daughter, Laura Michelle. 